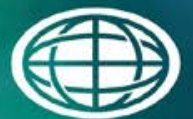

Savannah River

National Laboratory ${ }^{m}$

OPERATED BY SAVANNAH RIVER NUCLEAR SOLUTIONS

\title{
Chemical Composition and PCT Data for the Initial Set of Hanford Enhanced Waste Loading Glasses
}

K. M. Fox

T. B. Edwards

May 2014

SRNL-STI-2014-00063, Revision 0 


\section{DISCLAIMER}

This work was prepared under an agreement with and funded by the U.S. Government. Neither the U.S. Government or its employees, nor any of its contractors, subcontractors or their employees, makes any express or implied:

1. warranty or assumes any legal liability for the accuracy, completeness, or for the use or results of such use of any information, product, or process disclosed; or

2. representation that such use or results of such use would not infringe privately owned rights; or

3. endorsement or recommendation of any specifically identified commercial product, process, or service.

Any views and opinions of authors expressed in this work do not necessarily state or reflect those of the United States Government, or its contractors, or subcontractors.

\section{Printed in the United States of America \\ Prepared for U.S. Department of Energy}


Keywords: High level waste glass, durability

Retention: Permanent

\section{Chemical Composition and PCT Data for the Initial Set of Hanford Enhanced Waste Loading Glasses}

K. M. Fox

T. B. Edwards

May 2014

Prepared for the U.S. Department of Energy under 


\section{REVIEWS AND APPROVALS}

\section{AUTHORS:}

TECHNICAL REVIEW:

D. K. Peeler, Process Technology Programs, Reviewed per E7 2.60

APPROVAL:

D. H. McGuire, Manager

Date

Process Technology Programs

S. L. Marra, Manager

Date

Environmental \& Chemical Process Technology Research Programs 


\section{ACKNOWLEDGEMENTS}

The authors thank Phyllis Workman, Beverly Wall, Whitney Riley, and David Best at Savannah River National Laboratory for their assistance with the laboratory analyses described in this report. The authors thank Mike Schweiger and Renee Russell at the Pacific Northwest National Laboratory for helpful discussions and review of these data. 


\section{EXECUTIVE SUMMARY}

In this report, the Savannah River National Laboratory provides chemical analyses and Product Consistency Test results for 20 simulated high level waste glasses fabricated by the Pacific Northwest National Laboratory. The results of these analyses will be used as part of efforts to revise or extend the validation ranges of the current Hanford Waste Treatment and Immobilization Plant glass property models to cover a broader span of waste compositions.

The measured chemical composition data are reported and compared with the targeted values for each component for each glass. Two components of the study glasses, fluorine and silver, were not measured since each of these species would have required the use of an additional preparation method and their measured values were likely to be near or below analytical detection limits. Some of the glasses were difficult to prepare for chemical analysis. A sodium peroxide fusion dissolution method was successful in completely dissolving the glasses. Components present in the glasses in minor concentrations can be difficult to measure using this dissolution method due to dilution requirements. The use of a lithium metaborate preparation method for the minor components (planned for use since it is typically successful in digesting Defense Waste Processing Facility HLW glasses) resulted in an unacceptable amount of undissolved solids remaining in the sample solutions. An acid dissolution method was used instead, which provided more thorough dissolution of the glasses, although a small amount of undissolved material remained for some of the study glasses. The undissolved material was analyzed to determine those components of the glasses that did not fully dissolve. These components (e.g., calcium and chromium) were present in sufficient quantities to be reported from the measurements resulting from the sodium peroxide fusion preparation method, which did not leave undissolved material. Overall, the analyses resulted in sums of oxides that ranged from about 98 to $101.5 \mathrm{wt} \%$ for the study glasses, indicating excellent recovery of all the components in the chemical composition analyses.

Comparisons of the targeted and measured chemical compositions indicated that, in general, the measured values for the glasses met the targeted concentrations. Exceptions were $\mathrm{Cr}_{2} \mathrm{O}_{3}, \mathrm{MgO}$, and $\mathrm{P}_{2} \mathrm{O}_{5}$. The measured values for $\mathrm{Cr}_{2} \mathrm{O}_{3}$ were somewhat low when compared to the targeted values for all of the study glasses targeting $\mathrm{Cr}_{2} \mathrm{O}_{3}$ concentrations above $0.5 \mathrm{wt} \%$. Many of the measured $\mathrm{MgO}$ and $\mathrm{P}_{2} \mathrm{O}_{5}$ values were below the targeted values for those glasses that contained these components. Two of the study glasses exhibited differences from the targeted compositions that may indicate a batching error. Glasses EWG-HAI-Centroid-2 and EWG-OL-1672 had measured values for $\mathrm{Al}_{2} \mathrm{O}_{3}$ and $\mathrm{SiO}_{2}$ that were lower than the targeted values, and measured values for $\mathrm{B}_{2} \mathrm{O}_{3}$ that were higher than the targeted values. Glass EWGHAI-Centroid-2 also had a measured value for $\mathrm{Fe}_{2} \mathrm{O}_{3}$ that was lower than the targeted value.

A review of the PCT data, including standards and blanks, revealed no issues with the performance of the tests. The PCT results were normalized to both the targeted and measured compositions of the study glasses. Comparisons of the normalized PCT results for both the quenched and Canister Centerline Cooled versions of the study glasses are made with the Environmental Assessment benchmark glass for reference. 


\section{TABLE OF CONTENTS}

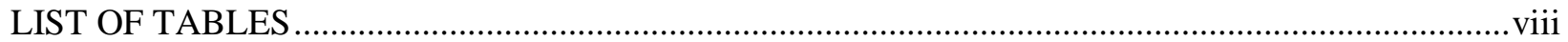

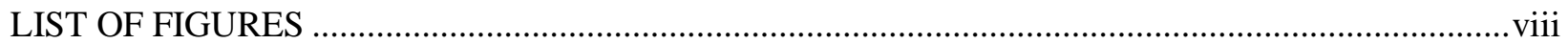

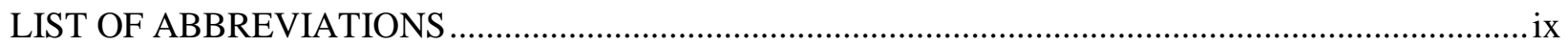

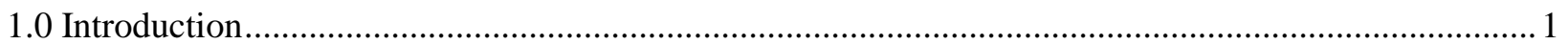

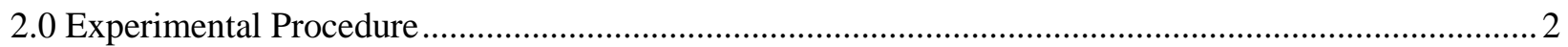

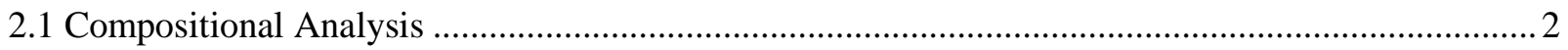

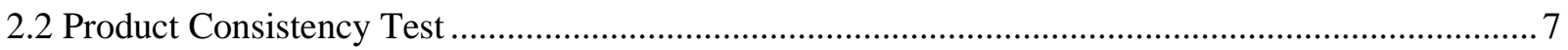

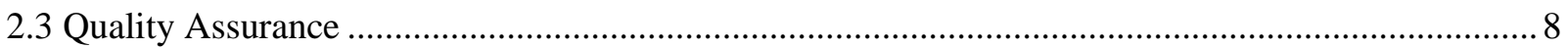

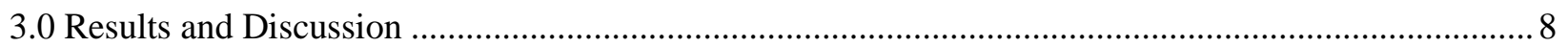

3.1 Statistical Review and Evaluation of Chemical Composition Measurements................................... 8

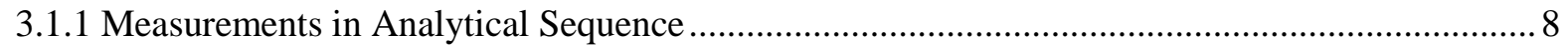

3.1.2 Composition Measurements by Glass Identifier...................................................................... 8

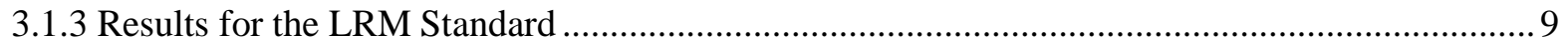

3.1.4 Measured versus Targeted Compositions .................................................................................... 9

3.2 Statistical Review and Evaluation of PCT Measurements …........................................................ 11

3.2.1 Measurements in Analytical Sequence ................................................................................... 11

3.2.2 Measurements by Glass Identifier .......................................................................................... 11

3.2.3 Results for the Samples of the Multi-Element Solution Standard .............................................12

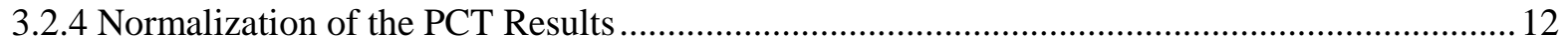

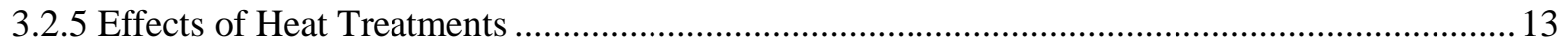

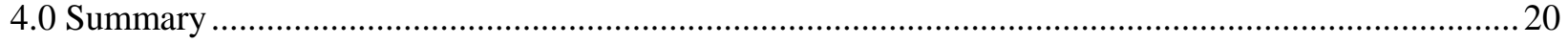

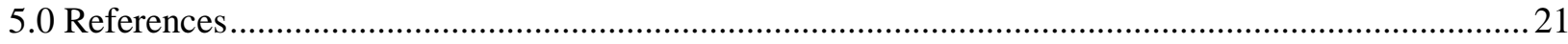

Appendix A . Tables and Exhibits Supporting the Chemical Composition Measurements......................A-1

Appendix B . Tables and Exhibits Supporting the PCT Results ............................................................ B-1 


\section{LIST OF TABLES}

Table 1-1. Identifiers for PNNL Glasses Characterized in this Study 2

Table 2-1. Preparation Methods Used in Reporting the Concentrations of Each of the Components of the

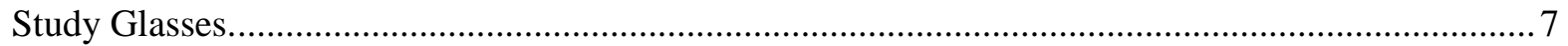

Table 3-1. Results from Samples of the Multi-Element Solution Standard ............................................. 12

Table 3-2. Common Logarithms of Normalized PCT Results............................................................. 14

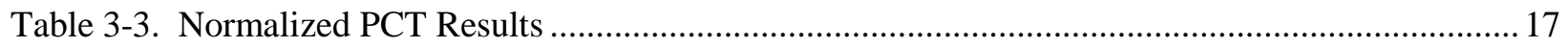

\section{LIST OF FIGURES}

Figure 2-1. SEM Micrograph of Undissolved Material from the AD Preparation of Glass EWG-OL-5155

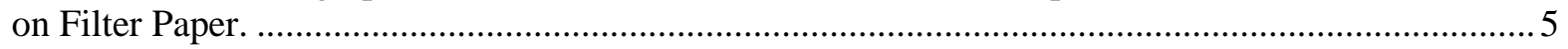

Figure 2-2. SEM Micrograph of Undissolved Material from the AD Preparation of Glass

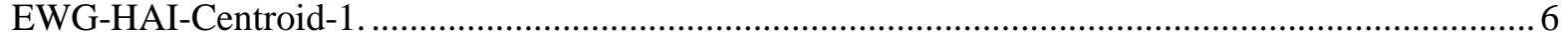

Figure 2-3. EDS Spectrum Corresponding to the White Rectangle Shown in Figure 2-2,........................ 6 


\section{LIST OF ABBREVIATIONS}

$\begin{array}{ll}\text { AD } & \text { Acid Dissolution } \\ \text { ANOVA } & \text { Analysis of Variance } \\ \text { ARM } & \text { Approved Reference Material } \\ \text { CCC } & \text { Canister Centerline Cooled } \\ \text { DOE } & \text { U.S. Department of Energy } \\ \text { EA } & \text { Environmental Assessment benchmark glass } \\ \text { EDS } & \text { Energy Dispersive Spectroscopy } \\ \text { ES-VSL } & \text { Energy Solutions - Vitreous State Laboratory } \\ \text { HLW } & \text { High Level Waste } \\ \text { ICP-OES } & \text { Inductively Coupled Plasma - Optical Emission Spectroscopy } \\ \text { LAW } & \text { Low Activity Waste } \\ \text { LRM } & \text { Low-level Reference Material } \\ \text { ORP } & \text { U.S. Department of Energy - Office of River Protection } \\ \text { PCT } & \text { Product Consistency Test } \\ \text { PF } & \text { Peroxide Fusion } \\ \text { PNNL } & \text { Pacific Northwest National Laboratory } \\ \text { SEM } & \text { Scanning Electron Microscopy } \\ \text { SRNL } & \text { Savannah River National Laboratory } \\ \text { TTQAP } & \text { Task Technical and Quality Assurance Plan } \\ \text { WTP } & \text { Hanford Waste Treatment and Immobilization Plant } \\ \end{array}$




\subsection{Introduction}

The U.S. Department of Energy (DOE) Office of River Protection (ORP) has requested that the Savannah River National Laboratory (SRNL) provide expert evaluation and experimental work in support of the River Protection Project vitrification technology development. DOE is building a Waste Treatment and Immobilization Plant (WTP) at the Hanford Site in Washington to remediate 55 million gallons of radioactive waste that is temporarily stored in 177 underground tanks. The low-activity waste (LAW) fraction will be partitioned from the high-level waste (HLW). Both the LAW and HLW will then be vitrified in borosilicate glass with Joule-heated ceramic melters (JHCM).

Efforts are being made to increase the loading of Hanford tank wastes in glass while maintaining an adequate ability to process, maintaining regulatory compliance, and maintaining product quality. DOE-ORP has requested that SRNL support the advancement of glass formulations and process control strategies in various key technical areas, as defined in the Task Technical and Quality Assurance Plan (TTQAP) supporting this work. ${ }^{1}$ One of these areas of current focus is enhanced HLW glass model applicability ranges.

The current WTP glass composition/properties models have been developed over a limited waste composition range. WTP has developed glass formulations, test data, and an operating strategy for processing the initial feed to be received. ${ }^{2}$ The glass composition region is sufficient for startup of the WTP with waste loadings at the minimum contract requirement. However, this composition region does not extend to glass compositions expected for a large fraction of tank wastes nor does it cover compositions from high waste loading glasses. The current WTP algorithms may only allow processing of the potential HLW compositions at significantly reduced waste loadings (see the recent summary of WTP glass properties models by Vienna et al. $^{3}$ ). To ensure applicability to the overall mission, DOE-ORP has implemented a program to expand the composition range of the models. ${ }^{4,5}$ New data will have to be generated in the glass regions of interest. Near term efforts for this task are focused on providing chemical composition analyses and chemical durability evaluations of HLW glasses formulated in new compositional regions of interest for WTP operation. These data will be used to support the expansion of the glass properties models.

In this report, SRNL provides chemical analyses and Product Consistency Test (PCT) results for select simulated HLW glasses fabricated by Pacific Northwest National Laboratory (PNNL). ${ }^{4}$ The results of these analyses will be used to revise or extend the validation ranges of the current WTP glass property models to cover a broader span of waste compositions. PNNL provided 20 glasses for this first set of analyses. The identifiers for the glasses are given in Table 1-1. Two versions of each glass were provided to support the analyses. Rapidly cooled (quenched) versions of each glass were used for chemical analyses and the PCTs. Canister Centerline Cooled (CCC) versions of each glass, which were heat treated to simulate slow cooling at the center of a WTP canister, were also used for the PCTs. 
SRNL-STI-2014-00063

Revision 0

Table 1-1. Identifiers for PNNL Glasses Characterized in this Study

\begin{tabular}{|c||}
\hline \multicolumn{1}{|c|}{ Glass Identifier } \\
\hline RSM-EWG-1-034 \\
\hline EWG-HAI-Centroid-1 \\
\hline EWG-HAI-Centroid-2 \\
\hline EWG-OL-2619 \\
\hline EWG-OL-38552 \\
\hline EWG-OL-15968 \\
\hline EWG-OL-33115 \\
\hline EWG-OL-3872 \\
\hline EWG-OL-1672 \\
\hline EWG-OL-801 \\
\hline EWG-OL-5155 \\
\hline EWG-OL-14827 \\
\hline EWG-OL-26012 \\
\hline EWG-OL-29285 \\
\hline EWG-OL-1369 \\
\hline EWG-OL-1580 \\
\hline EWG-OL-2463 \\
\hline EWG-OL-5801 \\
\hline EWG-OL-6198 \\
\hline EWG-OL-31644 \\
\hline
\end{tabular}

In the sections that follow, the methods used for measuring chemical composition and PCT performance are described and statistical reviews of the resulting data are provided. Detailed data from these analyses are included as appendices. The analytical plans developed in support of these analyses will be transmitted to PNNL for reference along with this report.

\subsection{Experimental Procedure}

\subsection{Compositional Analysis}

Chemical analysis was performed under the auspices of an analytical plan ${ }^{6}$ on a representative sample from the quenched version of each of the study glasses to allow for comparisons with the targeted compositions. Two preparation techniques, sodium peroxide fusion (PF) and acid dissolution (AD), were used to prepare the glass samples, in duplicate, for analysis. Each of the samples was analyzed, twice for each element of interest, by Inductively Coupled Plasma Optical Emission Spectroscopy (ICP-OES). Glass standards were also intermittently measured to assess the performance of the ICP-OES instrument over the course of these analyses. Specifically, several samples of the low-level reference material (LRM) ${ }^{7}$ were included as part of the analytical plan.

Two components of the study glasses, fluorine and silver, were not measured since each of these species would have required the use of an additional preparation method. ${ }^{a}$ Their targeted concentrations were also low, such that they were likely to be near or below analytical detection limits. After discussion with the PNNL client, it was determined that the effort needed to measure fluorine and silver was not worthwhile.

\footnotetext{
${ }^{a}$ Note that, as described in the analytical plan, measurement of the fluorine concentrations in the glasses was originally attempted. Undissolved solids remained in some of the sample solutions after separate potassium hydroxide preparations were performed specifically for determination of the fluorine concentrations. These difficulties with the preparation method contributed to the decision not to measure for fluorine.
} 
The PF method was selected for measurement of the major components of the glasses since SRNL experience has shown it to be an effective method for complete dissolution of simulated HLW glass samples. Measurement of the minor components using samples prepared with this method is difficult due to the high concentration of salts in the resulting solutions. A 10x dilution is required before analysis of the PF prepared solutions to avoid issues with extinguishing the plasma in the ICP-OES instrument. This dilution results in higher detection limits.

As described in the analytical plan, ${ }^{6}$ a lithium metaborate preparation method was originally selected and used to prepare samples of the glasses for measurement of the minor components, since the solutions prepared using this method do not require additional dilution prior to measurement. This preparation method typically results in complete dilution of simulated HLW glasses prepared in support of the Defense Waste Processing Facility (DWPF). However, undissolved solids were visible in the solutions after preparation of the EWG study glasses using this technique. The solutions prepared using the lithium metaborate method therefore were not measured. An acid dissolution preparation (AD) method was substituted for the lithium metaborate method, which resulted in fewer undissolved solids remaining in the solutions. However, some undissolved solids remained visible in the solutions for some of the study glasses.

Samples of some of the as-received, quenched EWG glasses were ground and analyzed via X-ray diffraction (XRD) to qualitatively determine the crystalline phases present which may have remained undissolved after the AD preparation method. An example XRD spectrum for glass EWG-OL-5155 is shown in Figure 2-1. Crystalline material in this glass was identified as trevorite $\left(\mathrm{NiFe}_{2} \mathrm{O}_{4}\right)$. Glass EWG-OL-6198 contained trevorite along with baddeleyite $\left(\mathrm{ZrO}_{2}\right)$ (Figure 2-2). Glass EGW-OL-15968 did not contain $\mathrm{Fe}_{2} \mathrm{O}_{3}$. Crystalline magnesiochromite $\left(\mathrm{MgCr}_{2} \mathrm{O}_{4}\right)$ was identified in this glass (Figure 2-3). As will be discussed further below, the elements contained in these phases are reported from the PF preparations rather than the AD preparations.

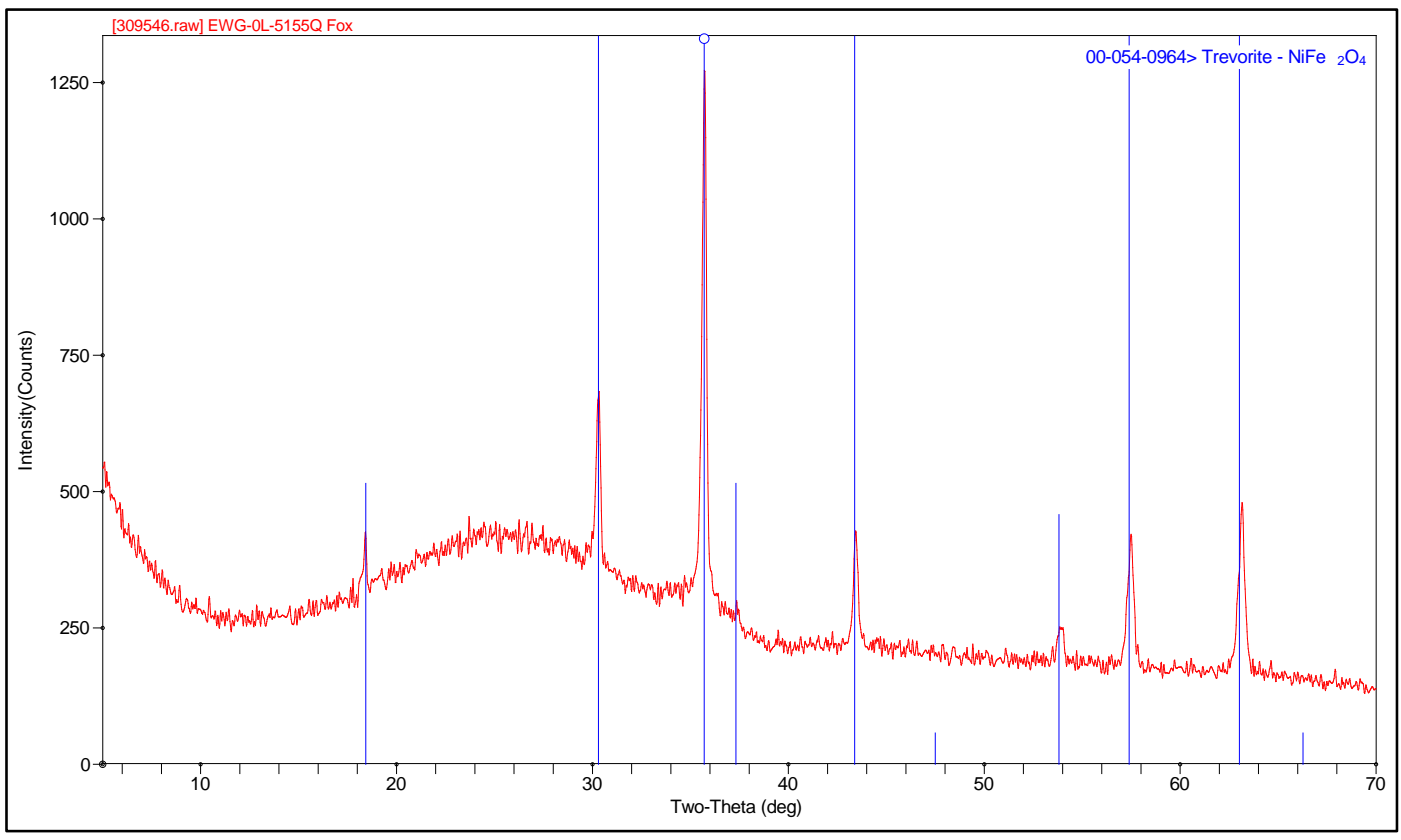

Figure 2-1. XRD spectrum from the as-received, quenched version of glass EWG-OL-5155. 


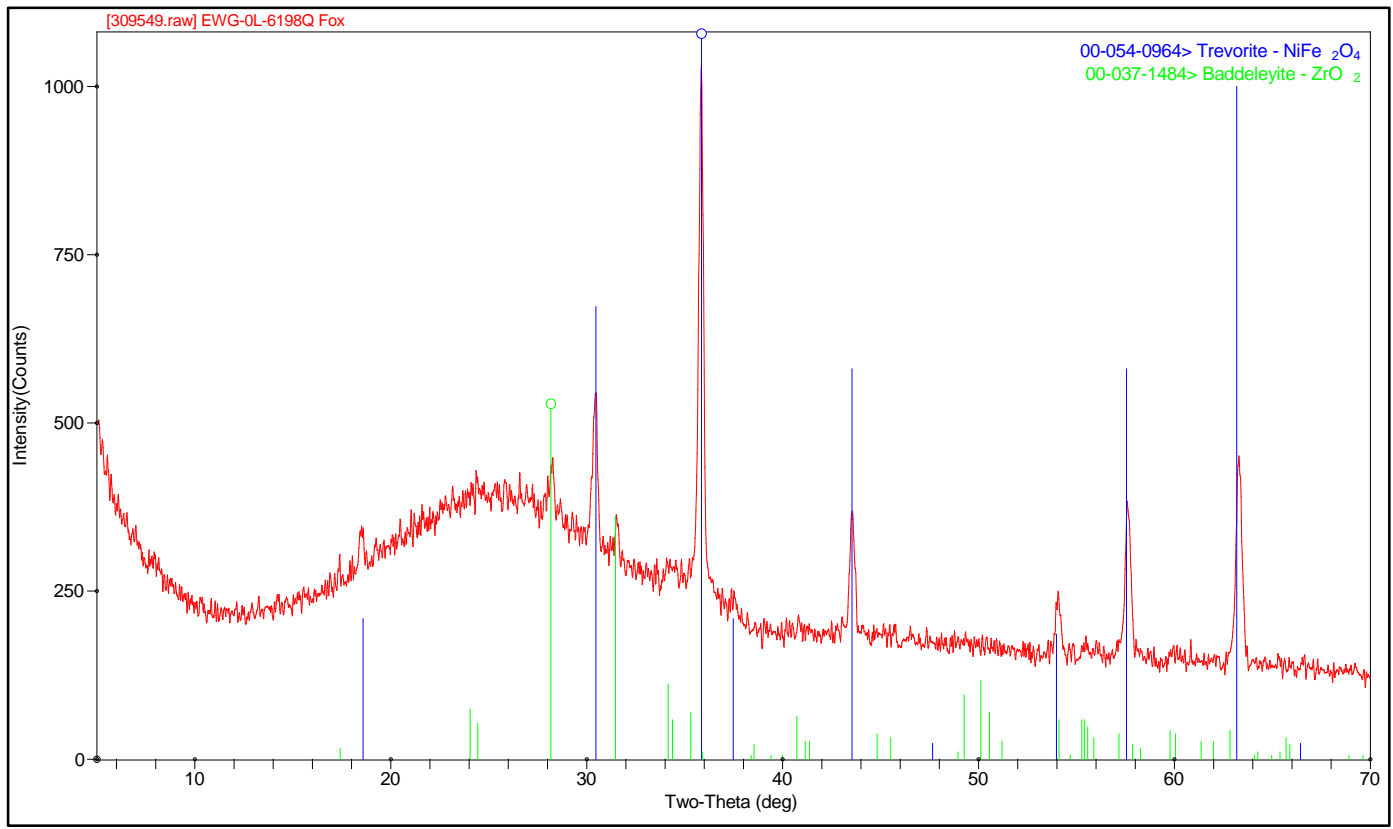

Figure 2-2. XRD spectrum from the as-received, quenched version of glass EWG-OL-6198.

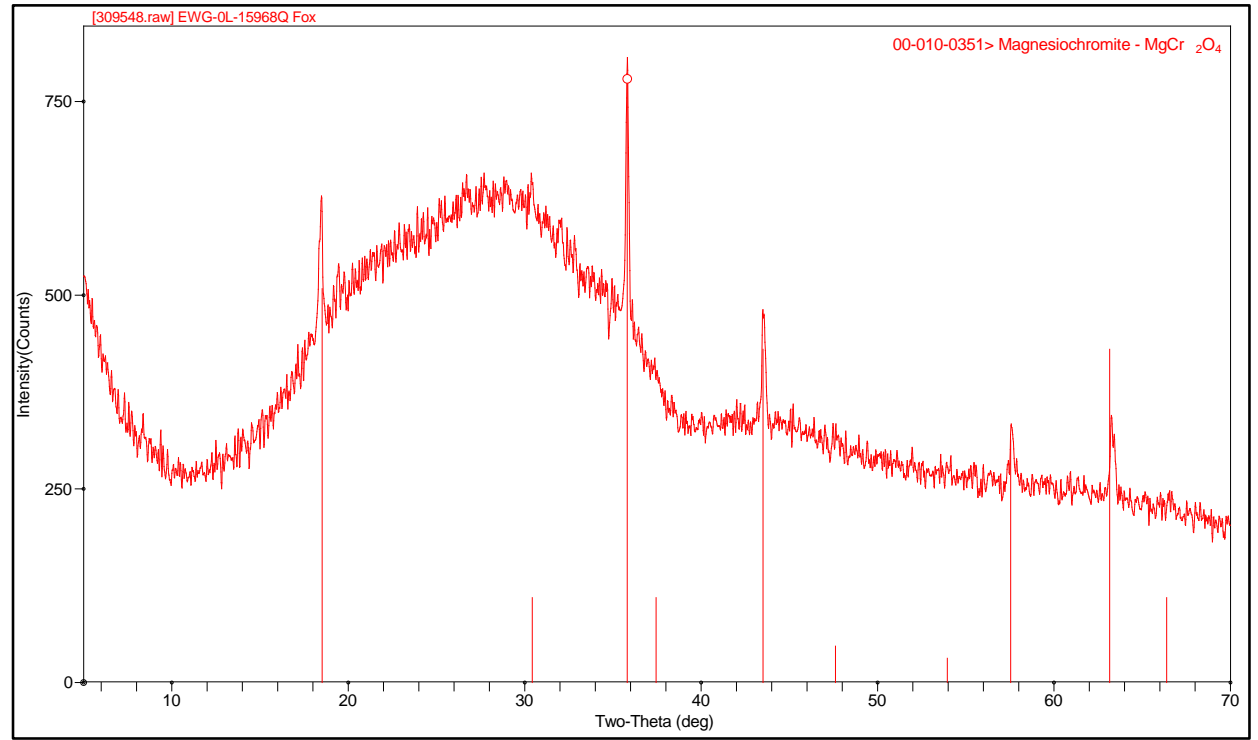

Figure 2-3. XRD spectrum from the as-received, quenched version of glass EWG-OL-15968.

The undissolved material from the $\mathrm{AD}$ prepared solutions was analyzed to determine those components of the glasses that did not dissolve. Samples of the undissolved material from several of the study glasses were filtered out of the solutions, dried, coated with a thin layer of carbon, and analyzed using Scanning Electron Microscopy (SEM) with Energy Dispersive Spectroscopy (EDS). An example SEM micrograph of the undissolved material from the AD preparation of glass EWG-OL-5155 captured on a filter is shown as Figure 2-1. A higher 
magnification image of undissolved material from the $\mathrm{AD}$ preparation of glass EWG-HAI-Centroid-1 is shown as Figure 2-2. The particulates exhibit a spinel morphology. The white rectangle in Figure 2-2 indicates the area scanned to produce the EDS spectrum shown as Figure 2-3. Elements observed include $\mathrm{Al}, \mathrm{Cr}, \mathrm{Fe}, \mathrm{Mg}, \mathrm{Mn}$, and $\mathrm{Ni}$. Other elements observed during the analyses but not indicated by the example in Figure 2-3 include $\mathrm{Ca}, \mathrm{Cl}$, and $\mathrm{F}$. The halides are residual from the $\mathrm{AD}$ preparation technique.

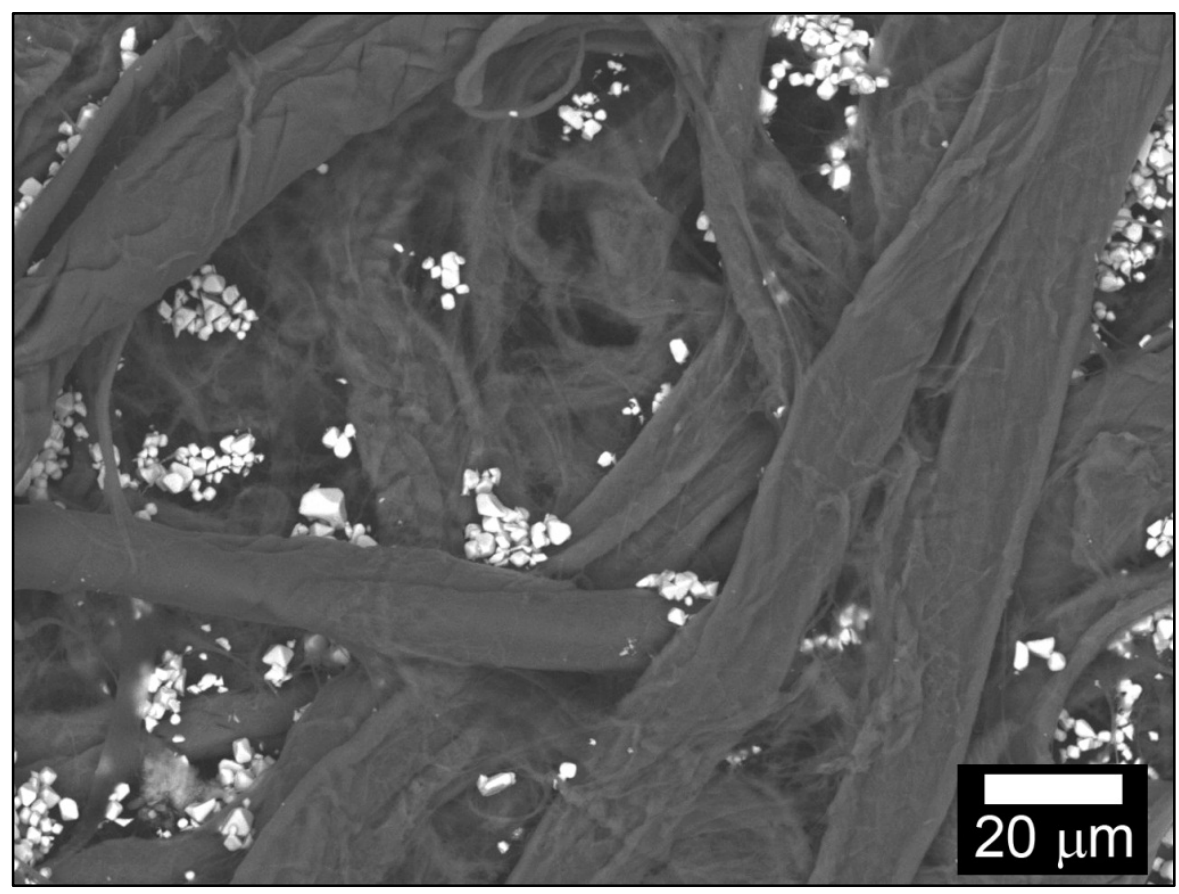

Figure 2-4. SEM Micrograph of Undissolved Material from the AD Preparation of Glass EWG-OL-5155 on Filter Paper. 


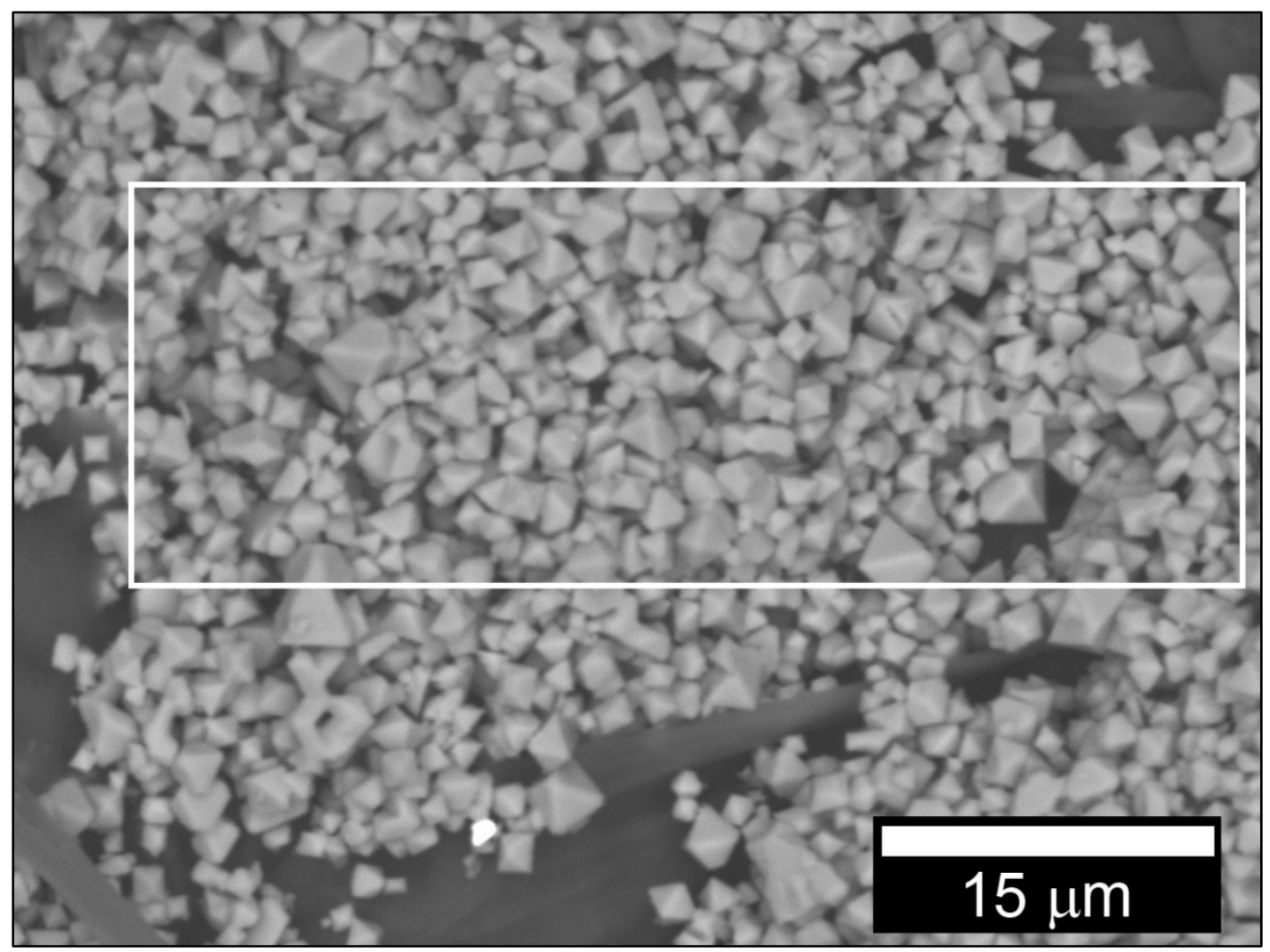

Figure 2-5. SEM Micrograph of Undissolved Material from the AD Preparation of Glass EWG-HAI-Centroid-1.

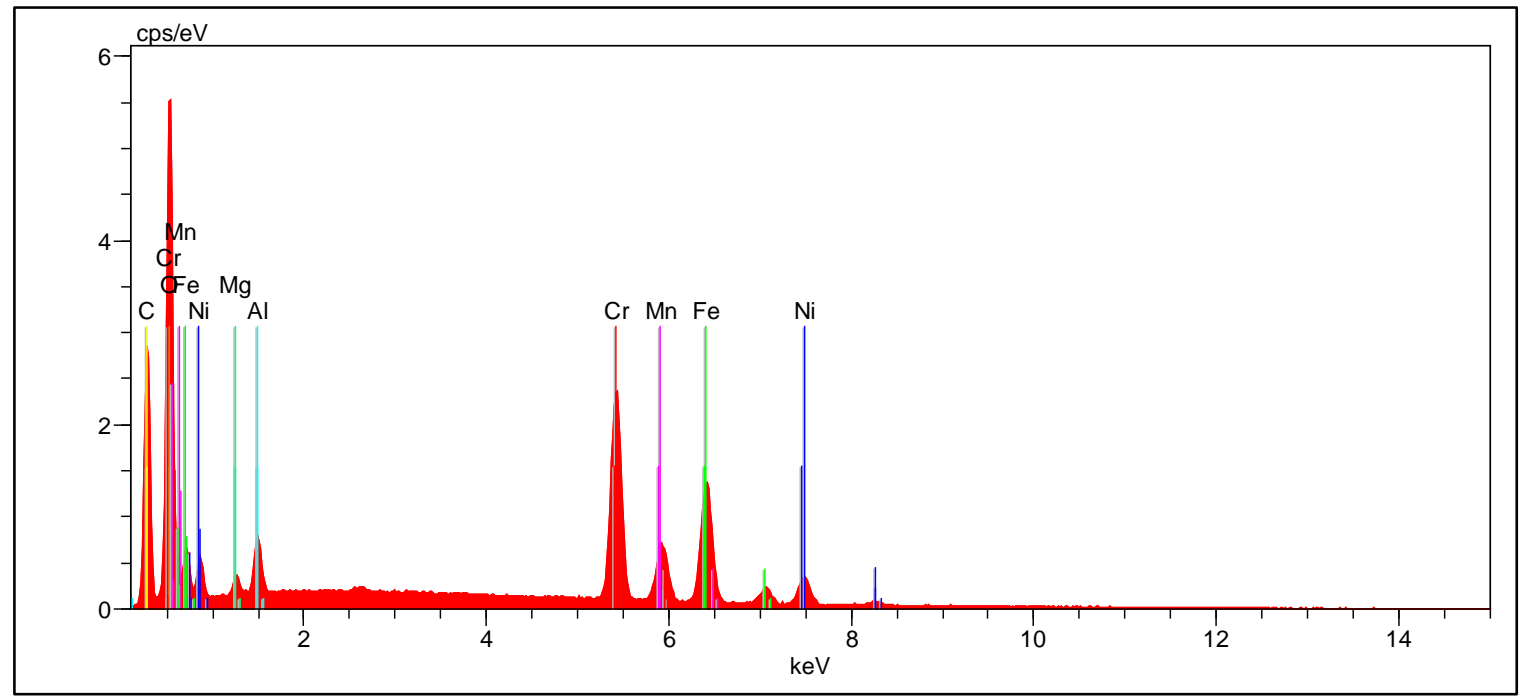

Figure 2-6. EDS Spectrum Corresponding to the White Rectangle Shown in Figure 2-2.

As a result of these analyses, the samples prepared with the PF method (which did not exhibit undissolved solids) were used in measuring and reporting the concentrations of $\mathrm{Ca}, \mathrm{Cr}$, and $\mathrm{Mg}$, 
in addition to the other major components of the glasses. ${ }^{\mathrm{a}}$ The targeted concentrations of these components in the study glasses were high enough that detection limits for the PF prepared solutions were not an issue. The preparation methods used for each of the reported glass components are listed in Table 2-1. As will be discussed later, the analyses resulted in sums of oxides that ranged from about 98 to $101.5 \mathrm{wt} \%$ for the study glasses. This indicates excellent recovery of all the components in the chemical composition analyses using the combination of $\mathrm{PF}$ and $\mathrm{AD}$ preparation methods.

\section{Table 2-1. Preparation Methods Used in Reporting the Concentrations of Each of the} Components of the Study Glasses.

\begin{tabular}{|c|c|}
\hline \hline Component & Preparation Method \\
\hline $\mathrm{Ag}$ & Not Reported \\
\hline $\mathrm{Al}$ & $\mathrm{PF}$ \\
\hline $\mathrm{B}$ & $\mathrm{PF}$ \\
\hline $\mathrm{Bi}$ & $\mathrm{AD}$ \\
\hline $\mathrm{Ca}$ & $\mathrm{PF}$ \\
\hline $\mathrm{Cd}$ & $\mathrm{AD}$ \\
\hline $\mathrm{Cr}$ & $\mathrm{PF}$ \\
\hline $\mathrm{F}$ & Not Reported \\
\hline $\mathrm{Fe}$ & $\mathrm{PF}$ \\
\hline $\mathrm{K}$ & $\mathrm{AD}$ \\
\hline $\mathrm{Li}$ & $\mathrm{PF}$ \\
\hline $\mathrm{Mg}$ & $\mathrm{PF}$ \\
\hline $\mathrm{Mn}$ & $\mathrm{PF}$ \\
\hline $\mathrm{Na}$ & $\mathrm{AD}$ \\
\hline $\mathrm{Ni}$ & $\mathrm{AD}$ \\
\hline $\mathrm{P}$ & $\mathrm{AD}$ \\
\hline $\mathrm{Pb}$ & $\mathrm{AD}$ \\
\hline $\mathrm{Ru}$ & $\mathrm{AD}$ \\
\hline $\mathrm{S}$ & $\mathrm{AD}$ \\
\hline $\mathrm{Si}$ & $\mathrm{PF}$ \\
\hline $\mathrm{Sr}$ & $\mathrm{AD}$ \\
\hline $\mathrm{Zr}$ & $\mathrm{AD}$ \\
\hline
\end{tabular}

\subsection{Product Consistency Test}

The PCT Method- $\mathrm{A}^{8}$ was performed in triplicate on each of the quenched and CCC versions of the study glasses to assess chemical durability. Also included in the experimental test matrix was the Environmental Assessment (EA) benchmark glass, ${ }^{9}$ the Approved Reference Material (ARM) glass, ${ }^{10}$ and blanks from the sample cleaning batch. Samples were ground, washed, and prepared according to the standard procedure. ${ }^{8}$ Fifteen milliliters of Type-I ASTM water were added to $1.5 \mathrm{~g}$ of glass in stainless steel vessels. The vessels were closed, sealed, and placed in an oven at $90 \pm 2{ }^{\circ} \mathrm{C}$ where the samples were maintained at temperature for 7 days. Once cooled, the resulting solutions were sampled (filtered and acidified), then labeled and analyzed by ICP-OES under the auspices of an analytical plan. ${ }^{11}$ Samples of a multi-element, standard solution were

\footnotetext{
${ }^{a}$ Nickel concentrations are reported from the samples prepared by the AD method since the PF solutions were not analyzed for nickel. After discussion with the PNNL client, it was determined that reporting the nickel concentrations from the samples prepared by the AD method would be sufficient despite the observation of nickel in the undissolved material since the targeted concentration of nickel in the study glasses is low ( $0.4 \mathrm{wt} \%$ for all glasses). Nickel will be measured from the samples prepared by the PF method in future studies of the EWG-series glasses.
} 
also included in the analytical plans as a check on the accuracy of the ICP-OES instrument used for these measurements. Normalized release rates were calculated based on the targeted (provided via email by PNNL) and measured compositions using the average of the common logarithms of the leachate concentrations.

\subsection{Quality Assurance}

Requirements for performing reviews of technical reports and the extent of review are established in manual E7 2.60. SRNL documents the extent and type of review using the SRNL Technical Report Design Checklist contained in WSRC-IM-2002-00011, Rev. 2.

\subsection{Results and Discussion}

\subsection{Statistical Review and Evaluation of Chemical Composition Measurements}

Table A-1 and Table A-2 in Appendix A provide the elemental concentration measurements in wt \% from the Set 1 study glasses that were analyzed by the AD method and Table A-3 in Appendix A provides the wt \% measurements from these glasses as analyzed by the PF dissolution method. These elemental concentrations were converted to oxide concentrations by multiplying the values for each element by the gravimetric factor for the corresponding oxide. Elemental measurements for samples of the LRM standard glass are also provided in the tables of Appendix A. During the process of converting to oxide concentrations, an elemental concentration that was reported to be below the detection limit of the analytical process used was set to the detection limit as the oxide concentration was determined.

In the sections that follow, the analytical sequences of the measurements are explored, the measurements of the LRM standard glass are investigated, the measurements for each glass are reviewed, the average chemical composition for each glass is determined, and comparisons are made between the measurements and the targeted compositions for the glasses. JMP Version 11.1.1 (SAS Institute, Inc.) ${ }^{12}$ was used to support these analyses.

\subsubsection{Measurements in Analytical Sequence}

Exhibit A-1 in Appendix A provides plots of the wt \% measurements generated for prepared samples by oxide and analytical block. The plots are in analytical sequence within each calibration block with different symbols and colors being used to represent each of the study and standard glasses. These plots include all of the measurement data from Table A-1, Table A-2, and Table A-3, with each plotted point identified by its Lab ID. While identifying patterns in these plots is difficult, there do not appear to be any gross patterns or trends in the analytical process over the course of these measurements. A better opportunity for a review of the measurements for each glass is provided in the discussion that follows.

\subsubsection{Composition Measurements by Glass Identifier}

Exhibit A-2 in Appendix A provides plots of the oxide concentration measurements by the PNNL Glass ID (including LRM as the reference standard) by Lab ID grouped by targeted concentration. Different symbols and colors are used to represent the different glasses. These plots show the individual measurements across the duplicates of each preparation method and the two instrument calibrations for each glass. A review of the plots presented in these exhibits reveals the repeatability of the four individual values for each oxide for each glass, which appears to be good across all of the oxides.

No issues of any practical importance are seen in these plots for the measurements across the duplicate preparations and the duplicate analyses. Thus, all of these data are to be used in 
determining representative, measured compositions of these glasses. However, the plots of Exhibit A-2 also provide an opportunity to compare measured against targeted values, and these comparisons indicate differences for some oxides for some of the study glasses. For example: the measured $\mathrm{Al}_{2} \mathrm{O}_{3}$ values for EWG-HAI-Centroid-2-Q are below the targeted concentration for this oxide for this glass and the measured $\mathrm{B}_{2} \mathrm{O}_{3}$ values for EWG-HAI-Centroid-2-Q and EWG-OL1672-Q are above their targeted values for this oxide for those glasses. A closer set of comparisons is provided in the discussions below. Note that a plot for $\mathrm{RuO}_{2}$ was omitted from Exhibit A-2 since all measured values for Ru were below the analytical detection limit.

\subsubsection{Results for the LRM Standard}

Exhibit A-3 in Appendix A provides statistical analyses of the LRM results by calibration block for each oxide of interest for both preparation methods. The results include analysis of variance (ANOVA) investigations, which determine statistically significant differences among the means of these groups for each of the oxides for the LRM standard. A statistically significant calibration effect on the block averages at the $5 \%$ significance level is present for the following components: ${ }^{\mathrm{a}}$

- AD preparation: $\mathrm{CdO}, \mathrm{K}_{2} \mathrm{O}, \mathrm{Na}_{2} \mathrm{O}, \mathrm{NiO}, \mathrm{P}_{2} \mathrm{O}_{5}, \mathrm{PbO}$, and $\mathrm{ZrO}_{2}$

- PF preparation: $\mathrm{Al}_{2} \mathrm{O}_{3}, \mathrm{CaO}, \mathrm{Cr}_{2} \mathrm{O}_{3}, \mathrm{Fe}_{2} \mathrm{O}_{3}$, and $\mathrm{SiO}_{2}$

Reference values for the oxide concentrations of the LRM standard are given in the header for each set of measurements in Exhibit A-3. Statistically significant calibration effects for a standard glass have been noted in previous glass variability studies for the DWPF that were conducted at SRNL ${ }^{13}$ and at Energy Solutions - Vitreous State Laboratory (ES-VSL). ${ }^{14}$ The observations of statistically significant calibration effects had no practical impact on the outcomes of those studies. In the following section, the results for the LRM standard are used to support the discussion of measured versus targeted compositions for the study glasses. As will be shown, the calibration effects have no practical influence on the outcome of this study.

\subsubsection{Measured versus Targeted Compositions}

From the discussion of Section 3.1.2, all of the measurements for each oxide for each glass (i.e., all of the measurements in Table A-1, Table A-2, and Table A-3) were averaged to determine a representative chemical composition for each glass. A sum of oxides was also computed for each glass based upon the measured values. Exhibit A-4 in Appendix A provides plots showing the result for each glass for each oxide to help highlight the comparisons between the measured and targeted values. Some observations from the plots of Exhibit A-4 are offered for the major oxides (i.e., those oxides in the study glasses or in LRM at a concentration of at least $0.5 \mathrm{wt} \%$ ):

- While the measured $\mathrm{Al}_{2} \mathrm{O}_{3}$ values for the majority of study glasses and for LRM are on target, the measured $\mathrm{Al}_{2} \mathrm{O}_{3}$ values for EWG-HAI-Centroid-2 and EWG-OL-1672 are low when compared to the targeted values for these glasses.

- While the measured $\mathrm{B}_{2} \mathrm{O}_{3}$ values for the majority of study glasses and for LRM are on target, the measured $\mathrm{B}_{2} \mathrm{O}_{3}$ values for EWG-HAI-Centroid-2 and EWG-OL-1672 are high when compared to the targeted values for these glasses.

\footnotetext{
a The 5\% significance level is used by SRNL for glass development work in support of the Defense Waste Processing Facility. Other significance levels may be used as considered appropriate for this study. The complete data are included in Exhibit A-3 to facilitate other interpretations.
} 
- The measured $\mathrm{Bi}_{2} \mathrm{O}_{3}$ value for EWG-OL-1672 is somewhat low and the measured $\mathrm{Bi}_{2} \mathrm{O}_{3}$ value for EWG-OL-5155 is somewhat high.

- The measured $\mathrm{CaO}$ value for EWG-OL-1672 is low when compared against the targeted $\mathrm{CaO}$ value for this glass. The measured $\mathrm{CaO}$ value for EWG-OL-31644 is somewhat high when compared against the targeted $\mathrm{CaO}$ value for this glass. The $\mathrm{CaO}$ value for LRM is slightly low, although the targeted $\mathrm{CaO}$ value for this glass is also low (0.5 wt \%).

- The measured $\mathrm{Cr}_{2} \mathrm{O}_{3}$ values are somewhat low when compared to the targeted values for all of the study glasses targeting $\mathrm{Cr}_{2} \mathrm{O}_{3}$ above $0.5 \mathrm{wt} \%$. The $\mathrm{Cr}_{2} \mathrm{O}_{3}$ value for LRM is close to the targeted value.

- The measured $\mathrm{Fe}_{2} \mathrm{O}_{3}$ values for EQG-OL-5155 and EWG-HAI-Centroid-2 are low. The $\mathrm{Fe}_{2} \mathrm{O}_{3}$ value for LRM is high, although a systematic effect for all the study glasses is not seen.

- There is some variability in the measured $\mathrm{K}_{2} \mathrm{O}$ values, and the values for EWG-OL-2619 and EWG-OL-33115 are somewhat low. The $\mathrm{K}_{2} \mathrm{O}$ value for LRM is close to the targeted value.

- The measured $\mathrm{MgO}$ values for those glasses that contained $\mathrm{MgO}$ are below the targeted values. The targeted MgO value for LRM is below the detection limit.

- The measured MnO value for EWG-OL-1672 is below the targeted value. The targeted $\mathrm{MnO}$ value for LRM is below the detection limit.

- There is some variability in the measured $\mathrm{Na}_{2} \mathrm{O}$ values. The measured value is high for LRM, although a systematic effect for all the study glasses is not seen.

- The measured $\mathrm{P}_{2} \mathrm{O}_{5}$ values are low for most of the glasses that targeted some amount of this component. This could be due to volatility of this component, depending upon the source used in batching the glasses. The $\mathrm{P}_{2} \mathrm{O}_{5}$ value for LRM is close to the targeted value.

- While the measured $\mathrm{SiO}_{2}$ values for the majority of study glasses and for LRM are on target, the measured $\mathrm{SiO}_{2}$ values for EWG-HAI-Centroid-2 and EWG-OL-1672 are low when compared to the targeted values for these glasses. The $\mathrm{SiO}_{2}$ value for $\mathrm{LRM}$ is close to the targeted value.

- While the measured $\mathrm{ZrO}_{2}$ values for the majority of study glasses and for LRM are on target, the measured $\mathrm{ZrO}_{2}$ values for EWG-OL-2619, EWG-OL-38552, and EWG-OL5801 are low when compared to the targeted values for these glasses. The $\mathrm{ZrO}_{2}$ value for LRM is close to the targeted value.

Table A-3 in Appendix A provides a summary of the average compositions as well as the targeted compositions and some associated differences and relative differences. All of the measured sums of oxides for the study glasses fall within the interval of 98 to $101.5 \mathrm{wt} \%$, indicating excellent recovery of all components. Entries in Table A-3 show the relative differences between the 
measured values and the targeted values. For the oxides with targeted values above zero, the relative differences are shaded if the values of the relative differences are $5 \%$ or more. $^{\text {a }}$

In summary, most of the observations above indicate differences among the targeted and measured compositions that have little or no practical significance. The two glasses whose measurements were most frequently off target (sometimes high and sometimes low) were EWGHAI-Centroid-2 and EWG-OL-1672. This may be due to an error in the batching of these glasses.

\subsection{Statistical Review and Evaluation of PCT Measurements}

Table B-1 in Appendix B provides the elemental leachate concentration measurements for the solution samples generated by the PCTs for the study glasses and standards. The values for these measurements are given in the table as-received ("ar") from the laboratory analyses and after adjustments. A few of the "ar" measurements were below the detection limit of 1 ppm (prior to correction for dilution). These measurements (indicated by a " $<$ ") were replaced by their detection limits in subsequent analyses. In addition to adjustments for detection limits, the values were adjusted for the dilution factors: study glasses, blanks, and the ARM glass were multiplied by 1.6667 to determine the values in parts per million (ppm) and the values for EA were multiplied by 16.6667. Based on the masses of the PCT vessels before and after the 7-day procedures, there was no water loss issue with any vessel during any of the PCT testing. The ratio of leachant volume to the mass of ground glass was confirmed to be correct for each vessel. All of the measurements of the ARM glass fell within the control ranges. ${ }^{10}$

The measured $\mathrm{pH}$ values for each of the PCT leachates are provided in Table B-2 in Appendix B for reference.

In the sections that follow, the analytical sequences of the measurements are explored, the measurements for each glass are reviewed, the measurements of the multi-element solution standard are investigated, the normalized PCTs for each glass are determined, and comparisons are made between the PCTs for the two heat treatments of each glass. JMP Version 9.0.0 (SAS Institute, Inc. $)^{15}$ was used to support these analyses.

\subsubsection{Measurements in Analytical Sequence}

Exhibit B-1 in Appendix B provides plots of the common logarithms of the leachate (ppm) concentrations in analytical sequence by analytical block by analytical set. Each of the two analytical sets corresponds to an oven run that was used to conduct the PCT measurements needed to support the study of this first set of PNNL glasses. No issues were observed in these plots.

\subsubsection{Measurements by Glass Identifier}

Exhibit B-2 in Appendix B provides plots of the leachate concentrations for both the quenched and CCC version of each of the study glasses and for the standards for each analytical set. These plots are in common logarithms of the ppm values and allow for the assessment of the repeatability of the measurements and any differences between the quenched and CCC version of a given glass. For some of the glasses, minor scatter among the triplicate values of some analytes is observed; however, these results are not expected to affect the outcome of this study. In addition, there are differences in the PCT responses between the quenched and CCC versions of some of the study glasses. A closer look at the quenched and CCC outcomes is provided below.

\footnotetext{
${ }^{\text {a }}$ The 5\% value was selected arbitrarily and does not necessarily indicate a difference of practical concern.
} 


\subsubsection{Results for the Samples of the Multi-Element Solution Standard}

Exhibit B-3 in Appendix B provides analyses of the measurements of the multi-element solution standard by analytical block for each analytical set, including ANOVA investigations for each element of interest. The reference value of the solution standard for each element of interest is also provided as part of the header information in the exhibit.

There was no statistically significant difference (at a 5\% level) among the averages of analytical blocks for these measurements for any of the analytes at positive concentrations for this standard. For reference, Table 3-1 provides the block averages, the reference values, and the percent differences between the measured and reference values for these measurements.

Table 3-1. Results from Samples of the Multi-Element Solution Standard

\begin{tabular}{|c|c|c|c|c|c|c|c|c|}
\hline $\begin{array}{c}\text { Analytical } \\
\text { Set }\end{array}$ & Block & $\begin{array}{c}\text { Mean } \\
\text { (B) } \\
\text { (ppm) }\end{array}$ & $\begin{array}{c}\text { Mean } \\
\text { (Ca) } \\
\text { (ppm) }\end{array}$ & $\begin{array}{c}\text { Mean } \\
(\mathbf{K}) \\
(\mathbf{p p m})\end{array}$ & $\begin{array}{c}\text { Mean } \\
\text { (Li) } \\
\text { (ppm) }\end{array}$ & $\begin{array}{c}\text { Mean } \\
\text { (Na) } \\
\text { (ppm) }\end{array}$ & $\begin{array}{c}\text { Mean } \\
\text { (P) } \\
\text { (ppm) }\end{array}$ & $\begin{array}{c}\text { Mean } \\
(\mathrm{Si}) \\
(\mathrm{ppm})\end{array}$ \\
\hline 1 & 1 & 21.00 & 1.00 & 10.21 & 10.38 & 79.87 & 1.00 & 50.03 \\
\hline 1 & 2 & 19.83 & 1.00 & 9.67 & 10.13 & 81.30 & 1.00 & 48.43 \\
\hline 1 & 3 & 20.50 & 1.00 & 9.97 & 10.33 & 81.70 & 1.00 & 48.73 \\
\hline 2 & 1 & 20.87 & 1.00 & 10.05 & 10.27 & 81.10 & 1.00 & 47.70 \\
\hline 2 & 2 & 20.73 & 1.00 & 10.37 & 10.47 & 81.70 & 1.00 & 49.07 \\
\hline 2 & 3 & 20.10 & 1.00 & 10.02 & 10.23 & 80.67 & 1.00 & 47.77 \\
\hline \multicolumn{2}{|c|}{ Grand Average } & 20.51 & 1.00 & 10.05 & 10.30 & 81.06 & 1.00 & 48.62 \\
\hline \multicolumn{2}{|c|}{ Reference Value } & 20.00 & 0.00 & 10.00 & 10.00 & 81.00 & 0.00 & 50.00 \\
\hline \multicolumn{2}{|c|}{ \% Relative Difference } & $2.5 \%$ & N/A & $0.5 \%$ & $3.0 \%$ & $0.1 \%$ & N/A & $-2.8 \%$ \\
\hline
\end{tabular}

\subsubsection{Normalization of the PCT Results}

For all of the PCT results, the PCT leachate concentrations were normalized using the targeted and measured compositions (wt \%) of the glasses to obtain a grams-per-liter (g/L) leachate concentration. As is the usual convention, the common logarithm of the normalized PCT (normalized leachate, NL) for each element of interest was determined and used for comparison. To accomplish this computation, one must:

1. Determine the common logarithm of the elemental leachate concentration (ppm) for each of the triplicates and each of the elements of interest (these values are provided in Table B-1 of Appendix B).

2. Average the common logarithms over the triplicates for each element of interest.

Normalizing using the measured composition:

3. Subtract the sum of 1 plus the common logarithm of the average cation measured concentration (expressed as a weight percent of the glass) from the average computed in step 2.

Or normalizing using the targeted composition:

3. Subtract a quantity equal to 1 plus the common logarithm of the targeted cation concentration (expressed as a weight percent of the glass) from the average computed in step 2. 


\subsubsection{Effects of Heat Treatments}

Exhibit B-4 in Appendix B provides plots of the normalized PCT responses for the two heat treatments for each of the study glasses as well as the responses for ARM and EA. The results are grouped by compositional view. These plots provide a comparison between the PCT responses for the two heat treatments of each study glass. Table 3-2 provides a listing of the normalized PCT responses in common logarithms of the g/L values and Table 3-3 provides the $\mathrm{g} / \mathrm{L}$ values themselves. In reading these tables, note that a single decimal point in a cell is used to indicate one of three conditions:

- The ARM glass does not contain $\mathrm{K}$

- The EA glass does not contain P

- A PCT result normalized to the targeted composition is not provided for those components with a targeted concentration of zero for a given glass composition. 
Table 3-2. Common Logarithms of Normalized PCT Results

\begin{tabular}{|c|c|c|c|c|c|c|c|c|c|}
\hline Glass ID & $\begin{array}{c}\text { Heat } \\
\text { Treatment }\end{array}$ & Normalization & $\begin{array}{c}\log \\
\text { NL[B (g/L)] }\end{array}$ & $\begin{array}{c}\log \\
\text { NL[Ca }(g / L)]\end{array}$ & $\begin{array}{c}\log \\
\text { NL[K (g/L)] }\end{array}$ & $\begin{array}{c}\log \\
\mathrm{NL}[\mathrm{Li}(\mathrm{g} / \mathrm{L})]\end{array}$ & $\begin{array}{c}\log \\
\text { NL[Na }(g / L)]\end{array}$ & $\begin{array}{c}\log \\
\mathrm{NL}[\mathbf{P}(\mathrm{g} / \mathrm{L})]\end{array}$ & $\begin{array}{c}\log \\
\mathrm{NL}[\mathrm{Si}(\mathrm{g} / \mathrm{L})]\end{array}$ \\
\hline ARM & ref & ref & -0.3277 & -0.9806 &. & -0.2371 & -0.3200 & -0.2310 & -0.5768 \\
\hline ARM & ref & ref & -0.3072 & -0.9393 & & -0.2666 & -0.3513 & -0.2158 & -0.6079 \\
\hline EA & ref & ref & 0.9101 & 0.3185 & 1.7006 & 0.7158 & 0.8045 &. & 0.3635 \\
\hline EA & ref & ref & 1.1951 & 0.3185 & 1.7006 & 0.9733 & 1.0838 & . & 0.5593 \\
\hline EWG-HAI-Centroid-1 & CCC & measured & -0.4507 & -1.2087 & -0.5223 & -0.4100 & -0.5437 & -0.0883 & -0.8556 \\
\hline EWG-HAI-Centroid-1 & $\mathrm{CCC}$ & targeted & -0.4554 & -1.1763 & -0.5340 & -0.4002 & -0.5293 & -0.1170 & -0.8541 \\
\hline EWG-HAI-Centroid-1 & quenched & measured & -0.3957 & -1.2087 & -0.4962 & -0.3046 & -0.4255 & -0.0883 & -0.6961 \\
\hline EWG-HAI-Centroid-1 & quenched & targeted & -0.4004 & -1.1763 & -0.5078 & -0.2949 & -0.4110 & -0.1170 & -0.6946 \\
\hline EWG-HAI-Centroid-2 & CCC & measured & 0.4491 & -1.1478 & -0.1981 & 0.4127 & 0.2912 & -0.3260 & -0.5679 \\
\hline EWG-HAI-Centroid-2 & $\mathrm{CCC}$ & targeted & 0.6292 & -1.1763 & -0.3549 & 0.3809 & 0.2530 & -0.4096 & -0.6032 \\
\hline EWG-HAI-Centroid-2 & quenched & measured & 0.3954 & -1.1478 & -0.2169 & 0.3728 & 0.2485 & -0.3344 & -0.6240 \\
\hline EWG-HAI-Centroid-2 & quenched & targeted & 0.5755 & -1.1763 & -0.3737 & 0.3410 & 0.2103 & -0.4180 & -0.6593 \\
\hline EWG-OL-1369 & CCC & measured & -0.5606 & -1.4298 & . & -0.4866 & -0.8139 & -0.8618 & -1.0197 \\
\hline EWG-OL-1369 & $\mathrm{CCC}$ & targeted & -0.5510 & -1.4330 & . & -0.4893 & -0.8138 & -0.8952 & -1.0194 \\
\hline EWG-OL-1369 & quenched & measured & -0.7230 & -1.5566 & . & -0.4911 & -0.8139 & -0.8618 & -1.0173 \\
\hline EWG-OL-1369 & quenched & targeted & -0.7134 & -1.5598 &. & -0.4937 & -0.8138 & -0.8952 & -1.0169 \\
\hline EWG-OL-14827 & CCC & measured & -0.2080 & -1.0573 & -0.2641 & -0.1296 & -0.1676 &. & -0.9055 \\
\hline EWG-OL-14827 & CCC & targeted & -0.2040 & -1.0713 & -0.2392 & -0.1284 & -0.1609 & . & -0.8956 \\
\hline EWG-OL-14827 & quenched & measured & -0.1533 & -1.0792 & -0.2113 & -0.0857 & -0.1178 & . & -0.8804 \\
\hline EWG-OL-14827 & quenched & targeted & -0.1494 & -1.0932 & -0.1864 & -0.0845 & -0.1111 &. & -0.8705 \\
\hline EWG-OL-1580 & CCC & measured & -0.5778 & -1.4513 &. & -0.5132 & -0.7955 & -0.8627 & -1.0041 \\
\hline EWG-OL-1580 & $\mathrm{CCC}$ & targeted & -0.5734 & -1.4416 & . & -0.5167 & -0.7813 & -0.8952 & -1.0120 \\
\hline EWG-OL-1580 & quenched & measured & -0.6385 & -1.5322 & . & -0.5069 & -0.7589 & -0.8627 & -1.0037 \\
\hline EWG-OL-1580 & quenched & targeted & -0.6341 & -1.5225 & . & -0.5104 & -0.7447 & -0.8952 & -1.0115 \\
\hline EWG-OL-15968 & CCC & measured & 1.0552 &. & . & 1.0198 & 0.8508 &. & -0.5643 \\
\hline EWG-OL-15968 & $\mathrm{CCC}$ & targeted & 1.0505 & . & . & 1.0191 & 0.8635 & . & -0.5608 \\
\hline EWG-OL-15968 & quenched & measured & 0.8456 & . & . & 0.8359 & 0.7054 & . & -0.3857 \\
\hline EWG-OL-15968 & quenched & targeted & 0.8409 & . & . & 0.8351 & 0.7181 & . & -0.3823 \\
\hline EWG-OL-1672 & CCC & measured & 0.0342 & -0.9583 & . &. & 0.0666 & -0.8004 & -1.2228 \\
\hline EWG-OL-1672 & $\mathrm{CCC}$ & targeted & 0.1840 & -1.0183 & . & . & 0.0236 & -0.8952 & -1.2788 \\
\hline EWG-OL-1672 & quenched & measured & -0.0002 & -0.9278 & . & . & 0.0405 & -0.8004 & -1.2636 \\
\hline EWG-OL-1672 & quenched & targeted & 0.1496 & -0.9878 & . & . & -0.0024 & -0.8952 & -1.3195 \\
\hline
\end{tabular}


Table 3-2. Common Logarithms of Normalized PCT Results (continued)

\begin{tabular}{|c|c|c|c|c|c|c|c|c|c|}
\hline Glass ID & $\begin{array}{c}\text { Heat } \\
\text { Treatment }\end{array}$ & Normalization & $\begin{array}{c}\log \\
\text { NL[B (g/L)] }\end{array}$ & $\begin{array}{c}\log \\
\text { NL[Ca }(g / L)]\end{array}$ & $\begin{array}{c}\log \\
\text { NL[K (g/L)] }\end{array}$ & $\begin{array}{c}\log \\
\mathrm{NL}[\mathrm{Li}(\mathrm{g} / \mathrm{L})]\end{array}$ & $\begin{array}{c}\log \\
\text { NL[Na }(g / L)]\end{array}$ & $\begin{array}{c}\log \\
\mathrm{NL}[\mathrm{P}(\mathrm{g} / \mathrm{L})]\end{array}$ & $\begin{array}{c}\log \\
\text { NL[Si (g/L)] }\end{array}$ \\
\hline EWG-OL-2463 & CCC & measured & 1.5127 & -0.7937 & 0.7035 & . & 1.4784 & -0.8555 & -0.7426 \\
\hline EWG-OL-2463 & CCC & targeted & 1.5197 & -0.8044 & 0.7167 & . & 1.4895 & -0.8952 & -0.7396 \\
\hline EWG-OL-2463 & quenched & measured & 1.1670 & -1.6187 & 0.9854 & . & 1.1302 & -0.8555 & -0.6091 \\
\hline EWG-OL-2463 & quenched & targeted & 1.1740 & -1.6294 & 0.9986 & & 1.1413 & -0.8952 & -0.6062 \\
\hline EWG-OL-26012 & CCC & measured & -0.2715 &. & -0.4251 & 0.7431 & 0.2341 & 1.0204 & -0.4599 \\
\hline EWG-OL-26012 & CCC & targeted & -0.2710 & $\cdot$ & -0.4068 & 0.7417 & 0.2372 & 0.9906 & -0.4619 \\
\hline EWG-OL-26012 & quenched & measured & -0.3485 & . & -0.7305 & -0.6690 & -0.2664 & -0.5386 & -0.5803 \\
\hline EWG-OL-26012 & quenched & targeted & -0.3479 & & -0.7123 & -0.6704 & -0.2633 & -0.5684 & -0.5824 \\
\hline EWG-OL-2619 & CCC & measured & -0.1409 & -0.9029 & -0.1547 &. & -0.0940 &. & -1.4200 \\
\hline EWG-OL-2619 & CCC & targeted & -0.1395 & -0.8990 & -0.2111 & . & -0.0954 & . & -1.4123 \\
\hline EWG-OL-2619 & quenched & measured & -0.1964 & -0.8983 & -0.2054 & . & -0.1539 & . & -1.4183 \\
\hline EWG-OL-2619 & quenched & targeted & -0.1950 & -0.8943 & -0.2617 & . & -0.1553 &. & -1.4106 \\
\hline EWG-OL-29285 & $\mathrm{CCC}$ & measured & -0.7335 & -1.3359 &. & . & -0.6169 & -0.8663 & -1.2118 \\
\hline EWG-OL-29285 & CCC & targeted & -0.7367 & -1.3270 & . & . & -0.6116 & -0.8952 & -1.2117 \\
\hline EWG-OL-29285 & quenched & measured & -0.6593 & -1.3105 & . & . & -0.6711 & -0.8663 & -1.2399 \\
\hline EWG-OL-29285 & quenched & targeted & -0.6625 & -1.3016 & . & . & -0.6658 & -0.8952 & -1.2399 \\
\hline EWG-OL-31644 & CCC & measured & -0.5767 & -1.5631 & . & -0.2935 & -0.7993 & . & -1.0924 \\
\hline EWG-OL-31644 & CCC & targeted & -0.5658 & -1.5410 & . & -0.2872 & -0.8060 & . & -1.0883 \\
\hline EWG-OL-31644 & quenched & measured & -0.4895 & -1.1317 & . & -0.5901 & -0.6151 & . & -1.1248 \\
\hline EWG-OL-31644 & quenched & targeted & -0.4786 & -1.1096 & . & -0.5838 & -0.6218 & & -1.1207 \\
\hline EWG-OL-33115 & CCC & measured & -0.4950 & -1.6226 & -0.6339 &. & -0.4551 & -0.8609 & -0.9095 \\
\hline EWG-OL-33115 & CCC & targeted & -0.4811 & -1.6323 & -0.6917 & . & -0.4441 & -0.8952 & -0.9058 \\
\hline EWG-OL-33115 & quenched & measured & -0.5106 & -1.5012 & -0.6239 & . & -0.4132 & -0.8609 & -1.0257 \\
\hline EWG-OL-33115 & quenched & targeted & -0.4967 & -1.5108 & -0.6818 & . & -0.4023 & -0.8952 & -1.0220 \\
\hline EWG-OL-38552 & CCC & measured & 1.2004 &. &. & 0.4301 & 0.9850 & -0.5107 & -1.5588 \\
\hline EWG-OL-38552 & CCC & targeted & 1.2139 & & . & 0.4286 & 0.9904 & -0.5495 & -1.5545 \\
\hline EWG-OL-38552 & quenched & measured & -0.1935 & . & . & -0.1157 & -0.4154 & -0.2247 & -0.6554 \\
\hline EWG-OL-38552 & quenched & targeted & -0.1801 & & . & -0.1172 & -0.4101 & -0.2635 & -0.6512 \\
\hline EWG-OL-3872 & $\mathrm{CCC}$ & measured & 1.5895 & -1.6164 & . & 1.2064 & 1.4185 & -0.4937 & 0.1395 \\
\hline EWG-OL-3872 & CCC & targeted & 1.5948 & -1.6225 & . & 1.1966 & 1.4320 & -0.5217 & 0.1335 \\
\hline EWG-OL-3872 & quenched & measured & 0.1483 & -1.4384 & . & 0.2397 & 0.3123 & -0.8672 & -0.2855 \\
\hline EWG-OL-3872 & quenched & targeted & 0.1536 & -1.4445 & . & 0.2300 & 0.3258 & -0.8952 & -0.2916 \\
\hline EWG-OL-5155 & $\mathrm{CCC}$ & measured & 0.8329 & 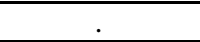 & . & 0.8147 & 0.6633 &. & -1.7577 \\
\hline
\end{tabular}


Table 3-2. Common Logarithms of Normalized PCT Results (continued)

\begin{tabular}{|c|c|c|c|c|c|c|c|c|c|}
\hline Glass ID & $\begin{array}{c}\text { Heat } \\
\text { Treatment }\end{array}$ & Normalization & $\begin{array}{c}\log \\
\mathrm{NL}[\mathrm{B}(\mathrm{g} / \mathrm{L})] \\
\end{array}$ & $\begin{array}{c}\log \\
\text { NL[Ca }(g / L)] \\
\end{array}$ & $\begin{array}{c}\log \\
\mathrm{NL}[\mathrm{K}(\mathrm{g} / \mathrm{L})] \\
\end{array}$ & $\begin{array}{c}\log \\
\mathrm{NL}[\mathrm{Li}(\mathrm{g} / \mathrm{L})] \\
\end{array}$ & $\begin{array}{c}\log \\
\mathrm{NL}[\mathrm{Na}(\mathrm{g} / \mathrm{L})] \\
\end{array}$ & $\begin{array}{c}\log \\
\mathrm{NL}[\mathbf{P}(\mathrm{g} / \mathrm{L})] \\
\end{array}$ & $\begin{array}{c}\log \\
\mathrm{NL}[\mathrm{Si}(\mathrm{g} / \mathrm{L})] \\
\end{array}$ \\
\hline EWG-OL-5155 & CCC & targeted & 0.8361 & . & . & 0.8167 & 0.6724 & . & -1.7489 \\
\hline EWG-OL-5155 & quenched & measured & 0.3106 & . & . & 0.2992 & 0.2209 & . & -0.6108 \\
\hline EWG-OL-5155 & quenched & targeted & 0.3137 & . & & 0.3013 & 0.2299 & & -0.6021 \\
\hline EWG-OL-5801 & CCC & measured & 0.7466 & . & 0.2290 & 0.6470 & 0.6517 & -0.0090 & -1.7308 \\
\hline EWG-OL-5801 & CCC & targeted & 0.7407 & . & 0.2477 & 0.6428 & 0.6596 & -0.0487 & -1.7338 \\
\hline EWG-OL-5801 & quenched & measured & 0.3880 & . & 0.0557 & 0.3683 & 0.3179 & 0.0993 & -0.6632 \\
\hline EWG-OL-5801 & quenched & targeted & 0.3821 & . & 0.0744 & 0.3641 & 0.3259 & 0.0597 & -0.6662 \\
\hline EWG-OL-6198 & CCC & measured & 0.8021 &. & 0.0088 & 0.6734 & 0.5783 & 0.2196 & -0.5426 \\
\hline EWG-OL-6198 & CCC & targeted & 0.8005 & . & 0.0131 & 0.6731 & 0.5746 & 0.1835 & -0.5395 \\
\hline EWG-OL-6198 & quenched & measured & 0.3355 & . & -0.1659 & 0.3123 & 0.2170 & -0.2144 & -0.6017 \\
\hline EWG-OL-6198 & quenched & targeted & 0.3340 & . & -0.1616 & 0.3119 & 0.2132 & -0.2504 & -0.5986 \\
\hline EWG-OL-801 & CCC & measured & 0.8185 & . &. &. & 0.7156 & 0.4082 & -0.6305 \\
\hline EWG-OL-801 & $\mathrm{CCC}$ & targeted & 0.8090 & . & . & . & 0.7122 & 0.3703 & -0.6362 \\
\hline EWG-OL-801 & quenched & measured & 0.7558 & . & . & . & 0.6713 & 0.2907 & -0.6441 \\
\hline EWG-OL-801 & quenched & targeted & 0.7464 &. & . & . & 0.6679 & 0.2529 & -0.6498 \\
\hline RSM-EWG-1-034 & CCC & measured & -0.3257 & -1.3864 & 0.3242 & -0.3379 & -0.3881 & -0.2594 & -0.7747 \\
\hline RSM-EWG-1-034 & CCC & targeted & -0.3439 & -1.3789 & 0.1566 & -0.3335 & -0.3852 & -0.2802 & -0.7577 \\
\hline RSM-EWG-1-034 & quenched & measured & -0.3700 & -1.3864 & 0.3242 & -0.3203 & -0.4453 & -0.3202 & -0.8642 \\
\hline RSM-EWG-1-034 & quenched & targeted & -0.3882 & -1.3789 & 0.1566 & -0.3159 & -0.4424 & -0.3411 & -0.8472 \\
\hline
\end{tabular}


Table 3-3. Normalized PCT Results

\begin{tabular}{|c|c|c|c|c|c|c|c|c|c|}
\hline Glass ID & $\begin{array}{c}\text { Heat } \\
\text { Treatment }\end{array}$ & Normalization & NL B(g/L) & NL Ca(g/L) & NL K(g/L) & NL Li (g/L) & NL Na (g/L) & NL P(g/L) & NL Si (g/L) \\
\hline ARM & ref & ref & 0.470 & 0.105 & . & 0.579 & 0.479 & 0.588 & 0.265 \\
\hline ARM & ref & ref & 0.493 & 0.115 & . & 0.541 & 0.445 & 0.608 & 0.247 \\
\hline EA & ref & ref & 8.130 & 2.082 & 50.192 & 5.198 & 6.375 &. & 2.310 \\
\hline EA & ref & ref & 15.670 & 2.082 & 50.192 & 9.403 & 12.127 & . & 3.625 \\
\hline EWG-HAI-Centroid-1 & CCC & measured & 0.354 & 0.062 & 0.300 & 0.389 & 0.286 & 0.816 & 0.139 \\
\hline EWG-HAI-Centroid-1 & CCC & targeted & 0.350 & 0.067 & 0.292 & 0.398 & 0.296 & 0.764 & 0.140 \\
\hline EWG-HAI-Centroid-1 & quenched & measured & 0.402 & 0.062 & 0.319 & 0.496 & 0.375 & 0.816 & 0.201 \\
\hline EWG-HAI-Centroid-1 & quenched & targeted & 0.398 & 0.067 & 0.311 & 0.507 & 0.388 & 0.764 & 0.202 \\
\hline EWG-HAI-Centroid-2 & CCC & measured & 2.813 & 0.071 & 0.634 & 2.587 & 1.955 & 0.472 & 0.270 \\
\hline EWG-HAI-Centroid-2 & CCC & targeted & 4.258 & 0.067 & 0.442 & 2.404 & 1.791 & 0.389 & 0.249 \\
\hline EWG-HAI-Centroid-2 & quenched & measured & 2.485 & 0.071 & 0.607 & 2.359 & 1.772 & 0.463 & 0.238 \\
\hline EWG-HAI-Centroid-2 & quenched & targeted & 3.762 & 0.067 & 0.423 & 2.193 & 1.623 & 0.382 & 0.219 \\
\hline EWG-OL-1369 & CCC & measured & 0.275 & 0.037 &. & 0.326 & 0.153 & 0.137 & 0.096 \\
\hline EWG-OL-1369 & CCC & targeted & 0.281 & 0.037 & . & 0.324 & 0.154 & 0.127 & 0.096 \\
\hline EWG-OL-1369 & quenched & measured & 0.189 & 0.028 & . & 0.323 & 0.153 & 0.137 & 0.096 \\
\hline EWG-OL-1369 & quenched & targeted & 0.193 & 0.028 &. & 0.321 & 0.154 & 0.127 & 0.096 \\
\hline EWG-OL-14827 & $\mathrm{CCC}$ & measured & 0.619 & 0.088 & 0.544 & 0.742 & 0.680 & . & 0.124 \\
\hline EWG-OL-14827 & CCC & targeted & 0.625 & 0.085 & 0.577 & 0.744 & 0.690 & . & 0.127 \\
\hline EWG-OL-14827 & quenched & measured & 0.703 & 0.083 & 0.615 & 0.821 & 0.762 & . & 0.132 \\
\hline EWG-OL-14827 & quenched & targeted & 0.709 & 0.081 & 0.651 & 0.823 & 0.774 & . & 0.135 \\
\hline EWG-OL-1580 & $\mathrm{CCC}$ & measured & 0.264 & 0.035 & . & 0.307 & 0.160 & 0.137 & 0.099 \\
\hline EWG-OL-1580 & CCC & targeted & 0.267 & 0.036 & . & 0.304 & 0.165 & 0.127 & 0.097 \\
\hline EWG-OL-1580 & quenched & measured & 0.230 & 0.029 & . & 0.311 & 0.174 & 0.137 & 0.099 \\
\hline EWG-OL-1580 & quenched & targeted & 0.232 & 0.030 & . & 0.309 & 0.180 & 0.127 & 0.097 \\
\hline EWG-OL-15968 & CCC & measured & 11.355 & . & . & 10.467 & 7.093 & . & 0.273 \\
\hline EWG-OL-15968 & CCC & targeted & 11.234 & . & . & 10.448 & 7.304 & . & 0.275 \\
\hline EWG-OL-15968 & quenched & measured & 7.008 & . & . & 6.853 & 5.075 & . & 0.411 \\
\hline EWG-OL-15968 & quenched & targeted & 6.933 & . & . & 6.841 & 5.226 & . & 0.415 \\
\hline EWG-OL-1672 & CCC & measured & 1.082 & 0.110 & . & 1.667 & 1.166 & 0.158 & 0.060 \\
\hline
\end{tabular}


Table 3-3. Normalized PCT Results (continued)

\begin{tabular}{|c|c|c|c|c|c|c|c|c|c|}
\hline Glass ID & $\begin{array}{c}\text { Heat } \\
\text { Treatment }\end{array}$ & Normalization & NL B(g/L) & NL Ca(g/L) & NL K(g/L) & NL Li (g/L) & NL Na (g/L) & NL P(g/L) & NL Si (g/L) \\
\hline EWG-OL-1672 & CCC & targeted & 1.528 & 0.096 & . & . & 1.056 & 0.127 & 0.053 \\
\hline EWG-OL-1672 & quenched & measured & 1.000 & 0.118 & . & . & 1.098 & 0.158 & 0.055 \\
\hline EWG-OL-1672 & quenched & targeted & 1.411 & 0.103 & . & . & 0.994 & 0.127 & 0.048 \\
\hline EWG-OL-2463 & CCC & measured & 32.560 & 0.161 & 5.053 & . & 30.089 & 0.139 & 0.181 \\
\hline EWG-OL-2463 & CCC & targeted & 33.090 & 0.157 & 5.209 & . & 30.871 & 0.127 & 0.182 \\
\hline EWG-OL-2463 & quenched & measured & 14.689 & 0.024 & 9.669 & . & 13.496 & 0.139 & 0.246 \\
\hline EWG-OL-2463 & quenched & targeted & 14.928 & 0.023 & 9.968 &. & 13.847 & 0.127 & 0.248 \\
\hline EWG-OL-26012 & CCC & measured & 0.535 &. & 0.376 & 5.535 & 1.714 & 10.481 & 0.347 \\
\hline EWG-OL-26012 & CCC & targeted & 0.536 & . & 0.392 & 5.516 & 1.727 & 9.787 & 0.345 \\
\hline EWG-OL-26012 & quenched & measured & 0.448 & . & 0.186 & 0.214 & 0.542 & 0.289 & 0.263 \\
\hline EWG-OL-26012 & quenched & targeted & 0.449 &. & 0.194 & 0.214 & 0.545 & 0.270 & 0.262 \\
\hline EWG-OL-2619 & CCC & measured & 0.723 & 0.125 & 0.700 &. & 0.805 &. & 0.038 \\
\hline EWG-OL-2619 & CCC & targeted & 0.725 & 0.126 & 0.615 & . & 0.803 & . & 0.039 \\
\hline EWG-OL-2619 & quenched & measured & 0.636 & 0.126 & 0.623 & . & 0.702 & . & 0.038 \\
\hline EWG-OL-2619 & quenched & targeted & 0.638 & 0.128 & 0.547 & . & 0.699 & . & 0.039 \\
\hline EWG-OL-29285 & CCC & measured & 0.185 & 0.046 &. & . & 0.242 & 0.136 & 0.061 \\
\hline EWG-OL-29285 & CCC & targeted & 0.183 & 0.047 & . & . & 0.245 & 0.127 & 0.061 \\
\hline EWG-OL-29285 & quenched & measured & 0.219 & 0.049 & . & . & 0.213 & 0.136 & 0.058 \\
\hline EWG-OL-29285 & quenched & targeted & 0.218 & 0.050 & . &. & 0.216 & 0.127 & 0.058 \\
\hline EWG-OL-31644 & CCC & measured & 0.265 & 0.027 & . & 0.509 & 0.159 &. & 0.081 \\
\hline EWG-OL-31644 & CCC & targeted & 0.272 & 0.029 & . & 0.516 & 0.156 & . & 0.082 \\
\hline EWG-OL-31644 & quenched & measured & 0.324 & 0.074 & . & 0.257 & 0.243 & . & 0.075 \\
\hline EWG-OL-31644 & quenched & targeted & 0.332 & 0.078 &. & 0.261 & 0.239 & . & 0.076 \\
\hline EWG-OL-33115 & CCC & measured & 0.320 & 0.024 & 0.232 &. & 0.351 & 0.138 & 0.123 \\
\hline EWG-OL-33115 & $\mathrm{CCC}$ & targeted & 0.330 & 0.023 & 0.203 & . & 0.360 & 0.127 & 0.124 \\
\hline EWG-OL-33115 & quenched & measured & 0.309 & 0.032 & 0.238 & . & 0.386 & 0.138 & 0.094 \\
\hline EWG-OL-33115 & quenched & targeted & 0.319 & 0.031 & 0.208 &. & 0.396 & 0.127 & 0.095 \\
\hline EWG-OL-38552 & CCC & measured & 15.865 & . & . & 2.692 & 9.661 & 0.309 & 0.028 \\
\hline EWG-OL-38552 & $\mathrm{CCC}$ & targeted & 16.363 & . & . & 2.683 & 9.780 & 0.282 & 0.028 \\
\hline
\end{tabular}


Table 3-3. Normalized PCT Results (continued)

\begin{tabular}{|c|c|c|c|c|c|c|c|c|c|}
\hline Glass ID & $\begin{array}{c}\text { Heat } \\
\text { Treatment }\end{array}$ & Normalization & NL B(g/L) & NL Ca(g/L) & NL K(g/L) & NL Li (g/L) & NL Na (g/L) & NL P(g/L) & NL Si (g/L) \\
\hline EWG-OL-38552 & quenched & measured & 0.641 & . & . & 0.766 & 0.384 & 0.596 & 0.221 \\
\hline EWG-OL-38552 & quenched & targeted & 0.661 & . & . & 0.763 & 0.389 & 0.545 & 0.223 \\
\hline EWG-OL-3872 & CCC & measured & 38.864 & 0.024 & . & 16.083 & 26.211 & 0.321 & 1.379 \\
\hline EWG-OL-3872 & CCC & targeted & 39.340 & 0.024 & . & 15.725 & 27.039 & 0.301 & 1.360 \\
\hline EWG-OL-3872 & quenched & measured & 1.407 & 0.036 & . & 1.737 & 2.053 & 0.136 & 0.518 \\
\hline EWG-OL-3872 & quenched & targeted & 1.424 & 0.036 & . & 1.698 & 2.118 & 0.127 & 0.511 \\
\hline EWG-OL-5155 & CCC & measured & 6.807 &. & . & 6.527 & 4.606 &. & 0.017 \\
\hline EWG-OL-5155 & $\mathrm{CCC}$ & targeted & 6.857 & . & . & 6.558 & 4.703 & . & 0.018 \\
\hline EWG-OL-5155 & quenched & measured & 2.044 & . & . & 1.992 & 1.663 & . & 0.245 \\
\hline EWG-OL-5155 & quenched & targeted & 2.059 & . & . & 2.001 & 1.698 &. & 0.250 \\
\hline EWG-OL-5801 & $\mathrm{CCC}$ & measured & 5.580 & . & 1.694 & 4.436 & 4.484 & 0.979 & 0.019 \\
\hline EWG-OL-5801 & CCC & targeted & 5.504 & . & 1.769 & 4.393 & 4.567 & 0.894 & 0.018 \\
\hline EWG-OL-5801 & quenched & measured & 2.443 & . & 1.137 & 2.335 & 2.079 & 1.257 & 0.217 \\
\hline EWG-OL-5801 & quenched & targeted & 2.410 & . & 1.187 & 2.312 & 2.118 & 1.147 & 0.216 \\
\hline EWG-OL-6198 & CCC & measured & 6.340 & . & 1.021 & 4.714 & 3.787 & 1.658 & 0.287 \\
\hline EWG-OL-6198 & CCC & targeted & 6.317 & . & 1.031 & 4.710 & 3.754 & 1.526 & 0.289 \\
\hline EWG-OL-6198 & quenched & measured & 2.165 & . & 0.683 & 2.052 & 1.648 & 0.610 & 0.250 \\
\hline EWG-OL-6198 & quenched & targeted & 2.158 & . & 0.689 & 2.051 & 1.634 & 0.562 & 0.252 \\
\hline EWG-OL-801 & CCC & measured & 6.584 & . & . &. & 5.195 & 2.560 & 0.234 \\
\hline EWG-OL-801 & CCC & targeted & 6.442 & . & . & . & 5.155 & 2.346 & 0.231 \\
\hline EWG-OL-801 & quenched & measured & 5.699 & . & . & . & 4.691 & 1.953 & 0.227 \\
\hline EWG-OL-801 & quenched & targeted & 5.577 &. &. &. & 4.655 & 1.790 & 0.224 \\
\hline RSM-EWG-1-034 & CCC & measured & 0.472 & 0.041 & 2.110 & 0.459 & 0.409 & 0.550 & 0.168 \\
\hline RSM-EWG-1-034 & $\mathrm{CCC}$ & targeted & 0.453 & 0.042 & 1.434 & 0.464 & 0.412 & 0.525 & 0.175 \\
\hline RSM-EWG-1-034 & quenched & measured & 0.427 & 0.041 & 2.110 & 0.478 & 0.359 & 0.478 & 0.137 \\
\hline RSM-EWG-1-034 & quenched & targeted & 0.409 & 0.042 & 1.434 & 0.483 & 0.361 & 0.456 & 0.142 \\
\hline
\end{tabular}




\subsection{Summary}

In this report, SRNL provides chemical analyses and PCT results for 20 simulated HLW glasses fabricated by PNNL. The results of these analyses will be used as part of efforts to revise or extend the validation ranges of the current WTP glass property models to cover a broader span of waste compositions.

The measured chemical composition data are reported and compared with the targeted values for each component for each glass. Two components of the study glasses, fluorine and silver, were not measured since each of these species would have required the use of an additional preparation method and their measured values were likely to be near or below analytical detection limits. Some of the glasses were difficult to prepare for chemical analysis. The use of a lithium metaborate preparation method (planned for use since it is typically successful in digesting DWPF HLW glasses) resulted in an unacceptable amount of undissolved solids remaining in the sample solutions. An acid dissolution method was used instead, which provided better dissolution of the glasses, although a small amount of undissolved material remained for some of the study glasses. The undissolved material was analyzed and found to consist of spinels. The elements present in the material that was left undissolved by the AD preparation method were reported using the PF dissolution method.

All of the measured sums of oxides for the study glasses fall within the interval of 98 to 101.5 wt\%. Comparisons of the targeted and measured chemical compositions indicated that, in general, the measured values for the glasses met the targeted concentrations. Exceptions were $\mathrm{Cr}_{2} \mathrm{O}_{3}, \mathrm{MgO}$, and $\mathrm{P}_{2} \mathrm{O}_{5}$. The measured values for $\mathrm{Cr}_{2} \mathrm{O}_{3}$ were somewhat low when compared to the targeted values for all of the study glasses targeting $\mathrm{Cr}_{2} \mathrm{O}_{3}$ concentrations above $0.5 \mathrm{wt} \%$. Many of the measured $\mathrm{MgO}$ and $\mathrm{P}_{2} \mathrm{O}_{5}$ values were below the targeted values for those glasses that contained these components. Two of the study glasses exhibited differences from the targeted compositions that may indicate a batching error. Glasses EWG-HAI-Centroid-2 and EWG-OL-1672 had measured values for $\mathrm{Al}_{2} \mathrm{O}_{3}$ and $\mathrm{SiO}_{2}$ that were lower than the targeted values, and measured values for $\mathrm{B}_{2} \mathrm{O}_{3}$ that were higher than the targeted values. Glass EWGHAI-Centroid-2 also had a measured value for $\mathrm{Fe}_{2} \mathrm{O}_{3}$ that was lower than the targeted value.

A review of the PCT data, including standards and blanks, revealed no issues with the performance of the tests. The PCT results were normalized to both the targeted and measured compositions of the study glasses. Comparisons of the normalized PCT results for both the quenched and CCC versions of the study glasses are made with the EA benchmark glass for reference.

Future analyses of the EWG-series glasses at SRNL will utilize the same dissolution methods described in this report. Spinel crystals that do not dissolve via the AD preparation method will be interrogated via SEM/EDS to ensure that the elements present in the undissolved material are reported from the PF prepared samples. Considerations should be made regarding the ability to dissolve and characterize spinels in WTP glasses as plans are made for analysis methods to be used during facility operation or waste qualification efforts. 


\subsection{References}

1. Fox, K. M. and D. K. Peeler, "Task Technical and Quality Assurance Plan for Hanford HLW Glass Development and Characterization," U.S. Department of Energy Report SRNL-RP-2013-00692, Revision 0, Savannah River National Laboratory, Aiken, SC (2013).

2. Vienna, J. D., "Preliminary IHLW Formulation Algorithm Description,” U.S. Department of Energy Report 24590-LAW-RPT-RT-04-0003, River Protection Project, Hanford Tank Waste Treatment and Immobilization Plant, Richland, WA (2005).

3. Vienna, J. D., D. C. Skorski, D. S. Kim, and J. Matyáš, “Glass Property Models and Constraints for Estimating the Glass to be Produced at Hanford by Implementing Current Advanced Glass Formulation Efforts," U.S. Department of Energy Report EWG-RPT-003, Revision 0, Pacific Northwest National Laboratory, Richland, WA (2013).

4. Vienna, J. D., D. S. Kim, M. J. Schweiger, J. S. McCloy, J. Matyáš, G. F. Piepel, and S. K. Cooley, “Test Plan: Enhanced Hanford Waste Glass Models," U.S. Department of Energy Report TP-EWG-00001, Revision 0, Pacific Northwest National Laboratory, Richland, WA (2013).

5. Muller, I. S., I. L. Pegg, and I. Joseph, "Test Plan: Enhanced LAW Glass Property-Composition Models, Phase 2," U.S. Department of Energy Report VSL-13T3050-1, Revision 0, Vitreous State Laboratory, Washington, DC (2013).

6. Peeler, D. K. and T. B. Edwards, "An Analytical Plan for Measuring the Chemical Compositions of an Initial Set of Glasses Supporting Hanford HLW Glass Development and Characterization," U.S. Department of Energy Memorandum SRNL-L3100-2013-00205, Savannah River National Laboratory, Aiken, SC (2013).

7. Ebert, W. L. and S. F. Wolfe, "Round-robin Testing of a Reference Glass for Low-Activity Waste Forms," U.S. Department of Energy Report ANL-99/22, Argonne National Laboratory, Argonne, IL (1999).

8. ASTM, "Standard Test Methods for Determining Chemical Durability of Nuclear Waste Glasses: The Product Consistency Test (PCT),” ASTM C-1285, (2002).

9. Jantzen, C. M., N. E. Bibler, D. C. Beam, C. L. Crawford, and M. A. Pickett, "Characterization of the Defense Waste Processing Facility (DWPF) Environmental Assessment (EA) Glass Standard Reference Material," U.S. Department of Energy Report WSRC-TR-92-346, Revision 1, Westinghouse Savannah River Company, Aiken, SC (1993).

10. Jantzen, C. M., J. B. Picket, K. G. Brown, T. B. Edwards, and D. C. Beam, "Process/Product Models for the Defense Waste Processing Facility (DWPF): Part I. Predicting Glass Durability from Composition Using a Thermodynamic Hydration Energy Reaction Model (THERMO)," U.S. Department of Energy Report WSRC-TR-93-672, Revision 1, Westinghouse Savannah River Company, Aiken, SC (1995).

11. Peeler, D. K. and T. B. Edwards, “An Analytical Plan for Measuring the PCT Solutions for an Initial Set of Glasses Supporting Hanford HLW Glass Development and Characterization,” U.S. Department of Energy Memorandum SRNL-L3100-2013-00206, Savannah River National Laboratory, Aiken, SC (2013).

12. JMP ${ }^{\mathrm{TM}}$, Ver. 11.1.1, [Computer Software] SAS Institute Inc., Cary, NC (2014). 
13. Peeler, D. K. and T. B. Edwards, "The Sludge Batch 7a Glass Variability Study with Frit 418 and Frit 702," U.S. Department of Energy Report SRNL-STI-2011-00063, Revision 0, Savannah River National Laboratory, Aiken, SC (2011).

14. Kot, W. K., I. L. Pegg, D. K. Peeler, and T. B. Edwards, "Sludge Batch 7a Glass Variability Study," U.S. Department of Energy Report VSL-11R2580-1, Revision 0, Vitreous State Laboratory, The Catholic University of America, Washington, D.C. (2012).

15. JMP $^{\mathrm{TM}}$, Ver. 9.0.0, [Computer Software] SAS Institute Inc., Cary, NC (2010). 
SRNL-STI-2014-00063

Revision 0

Appendix A. Tables and Exhibits Supporting the Chemical Composition Measurements 
Table A-1. AD Measurements of Set 1 Study Glasses - Part 1

\begin{tabular}{|c|c|c|c|c|c|c|c|c|c|}
\hline Glass ID & Block & Sub-Blk & Sequence & Lab ID & Bi (wt\%) & Cd (wt\%) & K (wt\%) & $\mathrm{Na}(\mathrm{wt} \%)$ & Ni (wt\%) \\
\hline LRM & 1 & 1 & 1 & LRMAD111 & $<0.100$ & 0.099 & 1.21 & 15.5 & 0.126 \\
\hline EWG-OL-3872-Q & 1 & 1 & 2 & P02AD11 & 2.80 & 0.074 & $<0.010$ & 13.7 & 0.289 \\
\hline EWG-OL-5801-Q & 1 & 1 & 3 & P08AD11 & $<0.100$ & 0.075 & 2.63 & 3.83 & 0.286 \\
\hline RSM-EWG-1-034-Q & 1 & 1 & 4 & P09AD11 & 0.968 & 0.014 & 0.076 & 7.04 & 0.117 \\
\hline EWG-OL-26012-Q & 1 & 1 & 5 & P03AD21 & 2.67 & 0.075 & 2.59 & 13.4 & 0.302 \\
\hline EWG-HAI-Centroid-1-Q & 1 & 1 & 6 & P12AD11 & 0.865 & 0.075 & 0.567 & 8.84 & 0.137 \\
\hline EWG-OL-5155-Q & 1 & 1 & 7 & P17AD11 & 2.93 & 0.077 & $<0.010$ & 3.75 & 0.134 \\
\hline EWG-OL-3872-Q & 1 & 1 & 8 & P02AD21 & 2.80 & 0.075 & $<0.010$ & 13.8 & 0.294 \\
\hline EWG-OL-5155-Q & 1 & 1 & 9 & P17AD21 & 2.93 & 0.076 & $<0.010$ & 3.80 & 0.135 \\
\hline EWG-OL-1672-Q & 1 & 1 & 10 & P18AD21 & 2.46 & 0.066 & $<0.010$ & 3.37 & 0.258 \\
\hline EWG-OL-33115-Q & 1 & 1 & 11 & P04AD11 & 2.76 & 0.074 & 2.21 & 5.08 & 0.295 \\
\hline LRM & 1 & 1 & 12 & LRMAD112 & $<0.100$ & 0.097 & 1.25 & 16.4 & 0.124 \\
\hline EWG-OL-5801-Q & 1 & 1 & 13 & P08AD21 & $<0.100$ & 0.076 & 2.63 & 3.82 & 0.287 \\
\hline EWG-OL-26012-Q & 1 & 1 & 14 & P03AD11 & 2.66 & 0.076 & 2.65 & 13.6 & 0.303 \\
\hline EWG-OL-38552-Q & 1 & 1 & 15 & P06AD21 & 2.74 & 0.075 & $<0.010$ & 3.79 & 0.290 \\
\hline EWG-OL-38552-Q & 1 & 1 & 16 & P06AD11 & 2.76 & 0.074 & $<0.010$ & 3.79 & 0.289 \\
\hline EWG-OL-33115-Q & 1 & 1 & 17 & P04AD21 & 2.73 & 0.073 & 2.22 & 5.08 & 0.293 \\
\hline EWG-HAI-Centroid-1-Q & 1 & 1 & 18 & P12AD21 & 0.863 & 0.075 & 0.574 & 8.90 & 0.137 \\
\hline RSM-EWG-1-034-Q & 1 & 1 & 19 & P09AD21 & 0.971 & 0.014 & 0.083 & 7.29 & 0.116 \\
\hline EWG-OL-14827-Q & 1 & 1 & 20 & P05AD11 & 2.62 & 0.070 & 2.69 & 3.84 & 0.223 \\
\hline EWG-OL-14827-Q & 1 & 1 & 21 & P05AD21 & 2.63 & 0.070 & 2.70 & 3.86 & 0.225 \\
\hline EWG-OL-1672-Q & 1 & 1 & 22 & P18AD11 & 2.42 & 0.065 & $<0.010$ & 3.41 & 0.256 \\
\hline LRM & 1 & 1 & 23 & LRMAD113 & $<0.100$ & 0.102 & 1.25 & 16.2 & 0.125 \\
\hline LRM & 1 & 2 & 1 & LRMAD111 & $<0.100$ & 0.109 & 1.23 & 16.1 & 0.115 \\
\hline EWG-OL-14827-Q & 1 & 2 & 2 & P05AD22 & 2.69 & 0.074 & 2.57 & 3.68 & 0.231 \\
\hline EWG-OL-26012-Q & 1 & 2 & 3 & P03AD22 & 2.72 & 0.077 & 2.58 & 13.4 & 0.305 \\
\hline EWG-OL-26012-Q & 1 & 2 & 4 & P03AD12 & 2.74 & 0.076 & 2.57 & 13.4 & 0.307 \\
\hline EWG-OL-38552-Q & 1 & 2 & 5 & P06AD22 & 2.79 & 0.076 & $<0.010$ & 3.71 & 0.295 \\
\hline RSM-EWG-1-034-Q & 1 & 2 & 6 & P09AD22 & 0.987 & 0.015 & 0.079 & 7.14 & 0.118 \\
\hline EWG-OL-38552-Q & 1 & 2 & 7 & P06AD12 & 2.77 & 0.076 & $<0.010$ & 3.73 & 0.292 \\
\hline EWG-OL-5801-Q & 1 & 2 & 8 & P08AD12 & $<0.100$ & 0.077 & 2.61 & 3.78 & 0.288 \\
\hline EWG-OL-3872-Q & 1 & 2 & 9 & P02AD22 & 2.80 & 0.075 & $<0.010$ & 13.8 & 0.291 \\
\hline EWG-OL-1672-Q & 1 & 2 & 10 & P18AD22 & 2.44 & 0.066 & $<0.010$ & 3.35 & 0.257 \\
\hline EWG-OL-5801-Q & 1 & 2 & 11 & P08AD22 & $<0.100$ & 0.078 & 2.53 & 3.68 & 0.289 \\
\hline LRM & 1 & 2 & 12 & LRMAD122 & $<0.100$ & 0.109 & 1.21 & 15.7 & 0.112 \\
\hline EWG-OL-5155-Q & 1 & 2 & 13 & P17AD22 & 2.98 & 0.077 & $<0.010$ & 3.74 & 0.136 \\
\hline RSM-EWG-1-034-Q & 1 & 2 & 14 & P09AD12 & 0.985 & 0.015 & 0.078 & 7.15 & 0.115 \\
\hline EWG-OL-33115-Q & 1 & 2 & 15 & P04AD22 & 2.81 & 0.074 & 2.14 & 4.91 & 0.295 \\
\hline EWG-OL-33115-Q & 1 & 2 & 16 & P04AD12 & 2.80 & 0.074 & 2.15 & 4.89 & 0.295 \\
\hline
\end{tabular}


Table A-1. AD Measurements of Set 1 Study Glasses - Part 1 (continued)

\begin{tabular}{|c|c|c|c|c|c|c|c|c|c|}
\hline Glass ID & Block & Sub-Blk & Sequence & Lab ID & $\mathrm{Bi}(\mathrm{wt} \%)$ & Cd (wt\%) & K (wt\%) & $\mathrm{Na}(\mathrm{wt} \%)$ & $\mathrm{Ni}(\mathrm{wt} \%)$ \\
\hline EWG-OL-14827-Q & 1 & 2 & 17 & P05AD12 & 2.68 & 0.072 & 2.59 & 3.69 & 0.226 \\
\hline EWG-HAI-Centroid-1-Q & 1 & 2 & 18 & P12AD22 & 0.886 & 0.076 & 0.559 & 8.73 & 0.137 \\
\hline EWG-OL-3872-Q & 1 & 2 & 19 & P02AD12 & 2.83 & 0.075 & $<0.010$ & 13.8 & 0.290 \\
\hline EWG-HAI-Centroid-1-Q & 1 & 2 & 20 & P12AD12 & 0.885 & 0.077 & 0.563 & 8.81 & 0.137 \\
\hline EWG-OL-5155-Q & 1 & 2 & 21 & P17AD12 & 3.00 & 0.078 & $<0.010$ & 3.86 & 0.134 \\
\hline EWG-OL-1672-Q & 1 & 2 & 22 & P18AD12 & 2.48 & 0.067 & $<0.010$ & 3.31 & 0.258 \\
\hline LRM & 1 & 2 & 23 & LRMAD123 & $<0.100$ & 0.108 & 1.23 & 16.1 & 0.114 \\
\hline LRM & 2 & 1 & 1 & LRMAD211 & $<0.100$ & 0.115 & 1.23 & 16.3 & 0.129 \\
\hline EWG-OL-1580-Q & 2 & 1 & 2 & P11AD11 & $<0.100$ & 0.075 & $<0.010$ & 3.84 & 0.292 \\
\hline EWG-HAI-Centroid-2-Q & 2 & 1 & 3 & P10AD11 & 0.789 & 0.068 & 0.396 & 7.69 & 0.117 \\
\hline EWG-OL-6198-Q & 2 & 1 & 4 & P16AD21 & $<0.100$ & 0.076 & 2.47 & 3.66 & 0.076 \\
\hline EWG-OL-1369-Q & 2 & 1 & 5 & P19AD21 & 2.66 & 0.075 & $<0.010$ & 3.65 & 0.291 \\
\hline EWG-OL-6198-Q & 2 & 1 & 6 & P16AD11 & $<0.100$ & 0.076 & 2.58 & 3.75 & 0.076 \\
\hline EWG-OL-31644-Q & 2 & 1 & 7 & P07AD21 & $<0.100$ & 0.074 & $<0.010$ & 3.70 & 0.225 \\
\hline EWG-OL-2463-Q & 2 & 1 & 8 & P15AD11 & $<0.100$ & 0.073 & 2.63 & 14.1 & 0.284 \\
\hline EWG-OL-1369-Q & 2 & 1 & 9 & P19AD11 & 2.65 & 0.075 & $<0.010$ & 3.85 & 0.289 \\
\hline EWG-OL-15968-Q & 2 & 1 & 10 & P14AD21 & $<0.100$ & 0.073 & $<0.010$ & 14.2 & 0.250 \\
\hline EWG-OL-29285-Q & 2 & 1 & 11 & P01AD21 & $<0.100$ & 0.076 & $<0.010$ & 3.89 & 0.295 \\
\hline LRM & 2 & 1 & 12 & LRMAD212 & $<0.100$ & 0.114 & 1.28 & 16.7 & 0.131 \\
\hline EWG-OL-2619-Q & 2 & 1 & 13 & P13AD11 & 2.80 & 0.076 & 2.29 & 3.82 & 0.235 \\
\hline EWG-OL-15968-Q & 2 & 1 & 14 & P14AD11 & $<0.100$ & 0.074 & $<0.010$ & 14.0 & 0.255 \\
\hline EWG-OL-1580-Q & 2 & 1 & 15 & P11AD21 & $<0.100$ & 0.076 & $<0.010$ & 3.83 & 0.291 \\
\hline EWG-HAI-Centroid-2-Q & 2 & 1 & 16 & P10AD21 & 0.791 & 0.068 & 0.430 & 8.13 & 0.117 \\
\hline EWG-OL-31644-Q & 2 & 1 & 17 & P07AD11 & $<0.100$ & 0.073 & $<0.010$ & 3.83 & 0.224 \\
\hline EWG-OL-29285-Q & 2 & 1 & 18 & P01AD11 & $<0.100$ & 0.075 & $<0.010$ & 3.83 & 0.294 \\
\hline EWG-OL-801-Q & 2 & 1 & 19 & P20AD21 & 2.67 & 0.075 & $<0.010$ & 13.2 & 0.295 \\
\hline EWG-OL-2463-Q & 2 & 1 & 20 & P15AD21 & $<0.100$ & 0.072 & 2.68 & 14.1 & 0.280 \\
\hline EWG-OL-801-Q & 2 & 1 & 21 & P20AD11 & 2.65 & 0.076 & $<0.010$ & 13.3 & 0.294 \\
\hline EWG-OL-2619-Q & 2 & 1 & 22 & P13AD21 & 2.79 & 0.077 & 2.24 & 3.77 & 0.236 \\
\hline LRM & 2 & 1 & 23 & LRMAD213 & $<0.100$ & 0.116 & 1.25 & 16.4 & 0.134 \\
\hline LRM & 2 & 2 & 1 & LRMAD221 & $<0.100$ & 0.108 & 1.18 & 15.5 & 0.121 \\
\hline EWG-OL-1580-Q & 2 & 2 & 2 & P11AD12 & $<0.100$ & 0.076 & $<0.010$ & 3.85 & 0.293 \\
\hline EWG-OL-15968-Q & 2 & 2 & 3 & P14AD12 & $<0.100$ & 0.073 & $<0.010$ & 13.3 & 0.254 \\
\hline EWG-OL-1369-Q & 2 & 2 & 4 & P19AD12 & 2.64 & 0.073 & $<0.010$ & 3.69 & 0.289 \\
\hline EWG-OL-2619-Q & 2 & 2 & 5 & P13AD22 & 2.77 & 0.077 & 2.10 & 3.59 & 0.236 \\
\hline EWG-HAI-Centroid-2-Q & 2 & 2 & 6 & P10AD12 & 0.794 & 0.068 & 0.395 & 7.68 & 0.117 \\
\hline EWG-OL-1369-Q & 2 & 2 & 7 & P19AD22 & 2.68 & 0.074 & $<0.010$ & 3.65 & 0.291 \\
\hline EWG-OL-2463-Q & 2 & 2 & 8 & P15AD22 & $<0.100$ & 0.073 & 2.46 & 13.2 & 0.284 \\
\hline EWG-OL-31644-Q & 2 & 2 & 9 & P07AD22 & $<0.100$ & 0.073 & $<0.010$ & 3.57 & 0.226 \\
\hline
\end{tabular}


Table A-1. AD Measurements of Set 1 Study Glasses - Part 1 (continued)

\begin{tabular}{|c|c|c|c|c|c|c|c|c|c|}
\hline Glass ID & Block & Sub-Blk & Sequence & Lab ID & $\mathrm{Bi}(\mathrm{wt} \%)$ & Cd (wt\%) & K (wt\%) & $\mathrm{Na}(\mathrm{wt} \%)$ & $\mathrm{Ni}(\mathrm{wt} \%)$ \\
\hline EWG-OL-801-Q & 2 & 2 & 10 & P20AD12 & 2.66 & 0.076 & $<0.010$ & 13.2 & 0.296 \\
\hline EWG-OL-31644-Q & 2 & 2 & 11 & P07AD12 & $<0.100$ & 0.074 & $<0.010$ & 3.51 & 0.226 \\
\hline LRM & 2 & 2 & 12 & LRMAD222 & $<0.100$ & 0.110 & 1.16 & 15.4 & 0.122 \\
\hline EWG-OL-801-Q & 2 & 2 & 13 & P20AD22 & 2.66 & 0.077 & $<0.010$ & 13.3 & 0.300 \\
\hline EWG-OL-29285-Q & 2 & 2 & 14 & P01AD12 & $<0.100$ & 0.075 & $<0.010$ & 3.64 & 0.296 \\
\hline EWG-OL-2463-Q & 2 & 2 & 15 & P15AD12 & $<0.100$ & 0.073 & 2.50 & 13.4 & 0.284 \\
\hline EWG-OL-1580-Q & 2 & 2 & 16 & P11AD22 & $<0.100$ & 0.075 & $<0.010$ & 3.81 & 0.294 \\
\hline EWG-OL-2619-Q & 2 & 2 & 17 & P13AD12 & 2.77 & 0.077 & 2.12 & 3.61 & 0.232 \\
\hline EWG-OL-29285-Q & 2 & 2 & 18 & P01AD22 & $<0.100$ & 0.076 & $<0.010$ & 3.66 & 0.297 \\
\hline EWG-OL-6198-Q & 2 & 2 & 19 & P16AD12 & $<0.100$ & 0.076 & 2.49 & 3.65 & 0.076 \\
\hline EWG-HAI-Centroid-2-Q & 2 & 2 & 20 & P10AD22 & 0.793 & 0.069 & 0.399 & 7.75 & 0.118 \\
\hline EWG-OL-15968-Q & 2 & 2 & 21 & P14AD22 & $<0.100$ & 0.073 & $<0.010$ & 13.5 & 0.255 \\
\hline EWG-OL-6198-Q & 2 & 2 & 22 & P16AD22 & $<0.100$ & 0.075 & 2.52 & 3.65 & 0.075 \\
\hline LRM & 2 & 2 & 23 & LRMAD223 & $<0.100$ & 0.110 & 1.17 & 15.5 & 0.122 \\
\hline
\end{tabular}


Table A-2. AD Measurements of Set 1 Study Glasses - Part 2

\begin{tabular}{|c|c|c|c|c|c|c|c|c|c|c|}
\hline Glass ID & Block & Sub-Blk & Sequence & Lab ID & P (wt\%) & $\mathrm{Pb}(\mathrm{wt} \%)$ & Ru (wt\%) & S (wt\%) & Sr (wt\%) & $\mathrm{Zr}(\mathrm{wt} \%)$ \\
\hline LRM & 1 & 1 & 1 & LRMAD111 & 0.246 & 0.072 & $<0.010$ & 0.099 & $<0.010$ & 0.664 \\
\hline EWG-OL-3872-Q & 1 & 1 & 2 & P02AD11 & 1.23 & 0.236 & $<0.010$ & 0.124 & 0.091 & $<0.100$ \\
\hline EWG-OL-5801-Q & 1 & 1 & 3 & P08AD11 & 1.19 & 0.254 & $<0.010$ & 0.129 & 0.098 & 2.41 \\
\hline RSM-EWG-1-034-Q & 1 & 1 & 4 & P09AD11 & 0.434 & 0.334 & $<0.010$ & 0.054 & $<0.010$ & 0.263 \\
\hline EWG-OL-26012-Q & 1 & 1 & 5 & P03AD21 & 1.21 & 0.257 & $<0.010$ & 0.095 & 0.099 & 2.93 \\
\hline EWG-HAI-Centroid-1-Q & 1 & 1 & 6 & P12AD11 & 0.204 & 0.249 & $<0.010$ & 0.101 & 0.099 & 0.664 \\
\hline EWG-OL-5155-Q & 1 & 1 & 7 & P17AD11 & $<0.100$ & 0.257 & $<0.010$ & 0.096 & 0.099 & 2.87 \\
\hline EWG-OL-3872-Q & 1 & 1 & 8 & P02AD21 & 1.23 & 0.243 & $<0.010$ & 0.124 & 0.093 & $<0.100$ \\
\hline EWG-OL-5155-Q & 1 & 1 & 9 & P17AD21 & $<0.100$ & 0.255 & $<0.010$ & 0.094 & 0.099 & 2.86 \\
\hline EWG-OL-1672-Q & 1 & 1 & 10 & P18AD21 & 1.05 & 0.226 & $<0.010$ & 0.014 & 0.093 & $<0.100$ \\
\hline EWG-OL-33115-Q & 1 & 1 & 11 & P04AD11 & 1.21 & 0.253 & $<0.010$ & 0.084 & 0.095 & $<0.100$ \\
\hline LRM & 1 & 1 & 12 & LRMAD112 & 0.230 & 0.072 & $<0.010$ & 0.114 & $<0.010$ & 0.657 \\
\hline EWG-OL-5801-Q & 1 & 1 & 13 & P08AD21 & 1.19 & 0.255 & $<0.010$ & 0.128 & 0.098 & 2.39 \\
\hline EWG-OL-26012-Q & 1 & 1 & 14 & P03AD11 & 1.22 & 0.258 & $<0.010$ & 0.098 & 0.099 & 2.91 \\
\hline EWG-OL-38552-Q & 1 & 1 & 15 & P06AD21 & 1.19 & 0.251 & $<0.010$ & 0.090 & 0.095 & 2.31 \\
\hline EWG-OL-38552-Q & 1 & 1 & 16 & P06AD11 & 1.19 & 0.250 & $<0.010$ & 0.090 & 0.095 & 2.32 \\
\hline EWG-OL-33115-Q & 1 & 1 & 17 & P04AD21 & 1.21 & 0.250 & $<0.010$ & 0.082 & 0.094 & $<0.100$ \\
\hline EWG-HAI-Centroid-1-Q & 1 & 1 & 18 & P12AD21 & 0.204 & 0.250 & $<0.010$ & 0.096 & 0.099 & 0.645 \\
\hline RSM-EWG-1-034-Q & 1 & 1 & 19 & P09AD21 & 0.435 & 0.335 & $<0.010$ & 0.053 & $<0.010$ & 0.261 \\
\hline EWG-OL-14827-Q & 1 & 1 & 20 & P05AD11 & $<0.100$ & 0.244 & $<0.010$ & 0.127 & 0.097 & $<0.100$ \\
\hline EWG-OL-14827-Q & 1 & 1 & 21 & P05AD21 & $<0.100$ & 0.245 & $<0.010$ & 0.123 & 0.096 & $<0.100$ \\
\hline EWG-OL-1672-Q & 1 & 1 & 22 & P18AD11 & 1.05 & 0.223 & $<0.010$ & 0.011 & 0.091 & $<0.100$ \\
\hline LRM & 1 & 1 & 23 & LRMAD113 & 0.246 & 0.067 & $<0.010$ & 0.113 & $<0.010$ & 0.647 \\
\hline LRM & 1 & 2 & 1 & LRMAD111 & 0.246 & 0.052 & $<0.010$ & 0.095 & $<0.010$ & 0.695 \\
\hline EWG-OL-14827-Q & 1 & 2 & 2 & P05AD22 & $<0.100$ & 0.252 & $<0.010$ & 0.126 & 0.097 & $<0.100$ \\
\hline EWG-OL-26012-Q & 1 & 2 & 3 & P03AD22 & 1.23 & 0.261 & $<0.010$ & 0.092 & 0.099 & 3.00 \\
\hline EWG-OL-26012-Q & 1 & 2 & 4 & P03AD12 & 1.23 & 0.261 & $<0.010$ & 0.093 & 0.100 & 3.01 \\
\hline EWG-OL-38552-Q & 1 & 2 & 5 & P06AD22 & 1.21 & 0.256 & $<0.010$ & 0.082 & 0.096 & 2.37 \\
\hline RSM-EWG-1-034-Q & 1 & 2 & 6 & P09AD22 & 0.439 & 0.342 & $<0.010$ & 0.050 & $<0.010$ & 0.291 \\
\hline EWG-OL-38552-Q & 1 & 2 & 7 & P06AD12 & 1.20 & 0.252 & $<0.010$ & 0.087 & 0.096 & 2.38 \\
\hline EWG-OL-5801-Q & 1 & 2 & 8 & P08AD12 & 1.20 & 0.257 & $<0.010$ & 0.129 & 0.098 & 2.45 \\
\hline EWG-OL-3872-Q & 1 & 2 & 9 & P02AD22 & 1.23 & 0.240 & $<0.010$ & 0.120 & 0.092 & $<0.100$ \\
\hline EWG-OL-1672-Q & 1 & 2 & 10 & P18AD22 & 1.05 & 0.225 & $<0.010$ & 0.007 & 0.091 & $<0.100$ \\
\hline EWG-OL-5801-Q & 1 & 2 & 11 & P08AD22 & 1.20 & 0.257 & $<0.010$ & 0.129 & 0.099 & 2.45 \\
\hline LRM & 1 & 2 & 12 & LRMAD122 & 0.225 & 0.055 & $<0.010$ & 0.057 & $<0.010$ & 0.690 \\
\hline EWG-OL-5155-Q & 1 & 2 & 13 & P17AD22 & $<0.100$ & 0.259 & $<0.010$ & 0.096 & 0.100 & 2.93 \\
\hline RSM-EWG-1-034-Q & 1 & 2 & 14 & P09AD12 & 0.439 & 0.337 & $<0.010$ & 0.051 & $<0.010$ & 0.288 \\
\hline EWG-OL-33115-Q & 1 & 2 & 15 & P04AD22 & 1.21 & 0.251 & $<0.010$ & 0.077 & 0.094 & $<0.100$ \\
\hline EWG-OL-33115-Q & 1 & 2 & 16 & P04AD12 & 1.21 & 0.251 & $<0.010$ & 0.082 & 0.093 & $<0.100$ \\
\hline
\end{tabular}


Table A-2. AD Measurements of Set 1 Study Glasses - Part 2 (continued)

\begin{tabular}{|c|c|c|c|c|c|c|c|c|c|c|}
\hline Glass ID & Block & Sub-Blk & Sequence & Lab ID & $P(w t \%)$ & $\mathrm{Pb}(\mathrm{wt} \%)$ & Ru (wt\%) & S (wt\%) & Sr (wt \%) & $\operatorname{Zr}(w t \%)$ \\
\hline EWG-OL-14827-Q & 1 & 2 & 17 & P05AD12 & $<0.100$ & 0.246 & $<0.010$ & 0.123 & 0.097 & $<0.100$ \\
\hline EWG-HAI-Centroid-1-Q & 1 & 2 & 18 & P12AD22 & 0.206 & 0.253 & $<0.010$ & 0.097 & 0.099 & 0.686 \\
\hline EWG-OL-3872-Q & 1 & 2 & 19 & P02AD12 & 1.22 & 0.237 & $<0.010$ & 0.122 & 0.091 & $<0.100$ \\
\hline EWG-HAI-Centroid-1-Q & 1 & 2 & 20 & P12AD12 & 0.203 & 0.252 & $<0.010$ & 0.096 & 0.098 & 0.699 \\
\hline EWG-OL-5155-Q & 1 & 2 & 21 & P17AD12 & $<0.100$ & 0.259 & $<0.010$ & 0.097 & 0.100 & 2.94 \\
\hline EWG-OL-1672-Q & 1 & 2 & 22 & P18AD12 & 1.06 & 0.226 & $<0.010$ & 0.010 & 0.090 & $<0.100$ \\
\hline LRM & 1 & 2 & 23 & LRMAD123 & 0.240 & 0.056 & $<0.010$ & 0.073 & $<0.010$ & 0.685 \\
\hline LRM & 2 & 1 & 1 & LRMAD211 & 0.191 & 0.069 & $<0.010$ & 0.081 & $<0.010$ & 0.714 \\
\hline EWG-OL-1580-Q & 2 & 1 & 2 & P11AD11 & 1.21 & 0.250 & $<0.010$ & 0.110 & 0.089 & 2.94 \\
\hline EWG-HAI-Centroid-2-Q & 2 & 1 & 3 & P10AD11 & 0.357 & 0.225 & $<0.010$ & 0.094 & 0.089 & 0.625 \\
\hline EWG-OL-6198-Q & 2 & 1 & 4 & P16AD21 & 1.20 & 0.256 & $<0.010$ & 0.123 & 0.097 & 2.76 \\
\hline EWG-OL-1369-Q & 2 & 1 & 5 & P19AD21 & 1.21 & 0.248 & $<0.010$ & 0.096 & 0.090 & 2.91 \\
\hline EWG-OL-6198-Q & 2 & 1 & 6 & P16AD11 & 1.21 & 0.259 & $<0.010$ & 0.129 & 0.100 & 2.75 \\
\hline EWG-OL-31644-Q & 2 & 1 & 7 & P07AD21 & $<0.100$ & 0.255 & $<0.010$ & 0.052 & 0.102 & $<0.100$ \\
\hline EWG-OL-2463-Q & 2 & 1 & 8 & P15AD11 & 1.20 & 0.250 & $<0.010$ & 0.133 & 0.098 & 2.89 \\
\hline EWG-OL-1369-Q & 2 & 1 & 9 & P19AD11 & 1.21 & 0.246 & $<0.010$ & 0.099 & 0.094 & 2.96 \\
\hline EWG-OL-15968-Q & 2 & 1 & 10 & P14AD21 & $<0.100$ & 0.251 & $<0.010$ & 0.117 & 0.097 & 2.95 \\
\hline EWG-OL-29285-Q & 2 & 1 & 11 & P01AD21 & 1.25 & 0.252 & $<0.010$ & 0.014 & 0.097 & 2.96 \\
\hline LRM & 2 & 1 & 12 & LRMAD212 & 0.188 & 0.069 & $<0.010$ & 0.123 & $<0.010$ & 0.699 \\
\hline EWG-OL-2619-Q & 2 & 1 & 13 & P13AD11 & $<0.100$ & 0.257 & $<0.010$ & 0.082 & 0.104 & 1.94 \\
\hline EWG-OL-15968-Q & 2 & 1 & 14 & P14AD11 & $<0.100$ & 0.254 & $<0.010$ & 0.124 & 0.098 & 2.92 \\
\hline EWG-OL-1580-Q & 2 & 1 & 15 & P11AD21 & 1.22 & 0.251 & $<0.010$ & 0.114 & 0.086 & 2.94 \\
\hline EWG-HAI-Centroid-2-Q & 2 & 1 & 16 & P10AD21 & 0.360 & 0.226 & $<0.010$ & 0.099 & 0.090 & 0.631 \\
\hline EWG-OL-31644-Q & 2 & 1 & 17 & P07AD11 & $<0.100$ & 0.254 & $<0.010$ & 0.050 & 0.101 & $<0.100$ \\
\hline EWG-OL-29285-Q & 2 & 1 & 18 & P01AD11 & 1.21 & 0.252 & $<0.010$ & 0.018 & 0.101 & 2.97 \\
\hline EWG-OL-801-Q & 2 & 1 & 19 & P20AD21 & 1.20 & 0.253 & $<0.010$ & 0.084 & 0.096 & $<0.100$ \\
\hline EWG-OL-2463-Q & 2 & 1 & 20 & P15AD21 & 1.18 & 0.247 & $<0.010$ & 0.129 & 0.099 & 2.90 \\
\hline EWG-OL-801-Q & 2 & 1 & 21 & P20AD11 & 1.19 & 0.255 & $<0.010$ & 0.083 & 0.097 & $<0.100$ \\
\hline EWG-OL-2619-Q & 2 & 1 & 22 & P13AD21 & $<0.100$ & 0.254 & $<0.010$ & 0.088 & 0.098 & 1.91 \\
\hline LRM & 2 & 1 & 23 & LRMAD213 & 0.195 & 0.073 & $<0.010$ & 0.105 & $<0.010$ & 0.702 \\
\hline LRM & 2 & 2 & 1 & LRMAD221 & 0.181 & 0.062 & $<0.010$ & 0.093 & $<0.010$ & 0.701 \\
\hline EWG-OL-1580-Q & 2 & 2 & 2 & P11AD12 & 1.22 & 0.251 & $<0.010$ & 0.114 & 0.092 & 2.92 \\
\hline EWG-OL-15968-Q & 2 & 2 & 3 & P14AD12 & $<0.100$ & 0.251 & $<0.010$ & 0.123 & 0.099 & 2.93 \\
\hline EWG-OL-1369-Q & 2 & 2 & 4 & P19AD12 & 1.21 & 0.245 & $<0.010$ & 0.100 & 0.095 & 2.95 \\
\hline EWG-OL-2619-Q & 2 & 2 & 5 & P13AD22 & $<0.100$ & 0.254 & $<0.010$ & 0.091 & 0.100 & 1.91 \\
\hline EWG-HAI-Centroid-2-Q & 2 & 2 & 6 & P10AD12 & 0.361 & 0.223 & $<0.010$ & 0.096 & 0.090 & 0.619 \\
\hline EWG-OL-1369-Q & 2 & 2 & 7 & P19AD22 & 1.22 & 0.245 & $<0.010$ & 0.100 & 0.091 & 2.93 \\
\hline EWG-OL-2463-Q & 2 & 2 & 8 & P15AD22 & 1.20 & 0.247 & $<0.010$ & 0.131 & 0.099 & 2.90 \\
\hline EWG-OL-31644-Q & 2 & 2 & 9 & P07AD22 & $<0.100$ & 0.253 & $<0.010$ & 0.050 & 0.102 & $<0.100$ \\
\hline
\end{tabular}


Table A-2. AD Measurements of Set 1 Study Glasses - Part 2 (continued)

\begin{tabular}{|c|c|c|c|c|c|c|c|c|c|c|}
\hline Glass ID & Block & Sub-Blk & Sequence & Lab ID & $P(w t \%)$ & $\mathrm{Pb}(\mathrm{wt} \%)$ & Ru (wt\%) & S (wt\%) & Sr (wt\%) & $\operatorname{Zr}(w t \%)$ \\
\hline EWG-OL-801-Q & 2 & 2 & 10 & P20AD12 & 1.20 & 0.255 & $<0.010$ & 0.087 & 0.098 & $<0.100$ \\
\hline EWG-OL-31644-Q & 2 & 2 & 11 & P07AD12 & $<0.100$ & 0.255 & $<0.010$ & 0.048 & 0.102 & $<0.100$ \\
\hline LRM & 2 & 2 & 12 & LRMAD222 & 0.185 & 0.071 & $<0.010$ & 0.120 & $<0.010$ & 0.690 \\
\hline EWG-OL-801-Q & 2 & 2 & 13 & P20AD22 & 1.21 & 0.258 & $<0.010$ & 0.087 & 0.098 & $<0.100$ \\
\hline EWG-OL-29285-Q & 2 & 2 & 14 & P01AD12 & 1.22 & 0.252 & $<0.010$ & 0.020 & 0.102 & 2.96 \\
\hline EWG-OL-2463-Q & 2 & 2 & 15 & P15AD12 & 1.20 & 0.249 & $<0.010$ & 0.129 & 0.099 & 2.86 \\
\hline EWG-OL-1580-Q & 2 & 2 & 16 & P11AD22 & 1.21 & 0.251 & $<0.010$ & 0.110 & 0.087 & 2.91 \\
\hline EWG-OL-2619-Q & 2 & 2 & 17 & P13AD12 & $<0.100$ & 0.254 & $<0.010$ & 0.085 & 0.099 & 1.90 \\
\hline EWG-OL-29285-Q & 2 & 2 & 18 & P01AD22 & 1.22 & 0.254 & $<0.010$ & 0.020 & 0.106 & 2.94 \\
\hline EWG-OL-6198-Q & 2 & 2 & 19 & P16AD12 & 1.20 & 0.258 & $<0.010$ & 0.126 & 0.098 & 2.72 \\
\hline EWG-HAI-Centroid-2-Q & 2 & 2 & 20 & P10AD22 & 0.362 & 0.228 & $<0.010$ & 0.099 & 0.092 & 0.609 \\
\hline EWG-OL-15968-Q & 2 & 2 & 21 & P14AD22 & $<0.100$ & 0.252 & $<0.010$ & 0.119 & 0.099 & 2.94 \\
\hline EWG-OL-6198-Q & 2 & 2 & 22 & P16AD22 & 1.21 & 0.255 & $<0.010$ & 0.125 & 0.099 & 2.74 \\
\hline LRM & 2 & 2 & 23 & LRMAD223 & 0.198 & 0.070 & $<0.010$ & 0.132 & $<0.010$ & 0.684 \\
\hline
\end{tabular}


Table A-3. PF Measurements of Set 1 Study Glasses

\begin{tabular}{|c|c|c|c|c|c|c|c|c|c|c|c|c|c|}
\hline Glass ID & Block & Sub-Blk & Sequence & Lab ID & $\begin{array}{c}\text { Al } \\
\text { (wt\%) }\end{array}$ & $\begin{array}{c}\text { B } \\
\text { (wt\%) }\end{array}$ & $\begin{array}{c}\text { Ca } \\
\text { (wt\%) }\end{array}$ & $\begin{array}{c}\mathrm{Cr} \\
\text { (wt\%) }\end{array}$ & $\begin{array}{c}\mathbf{F e} \\
(\mathrm{wt} \%)\end{array}$ & $\begin{array}{c}\mathbf{L i} \\
\text { (wt\%) }\end{array}$ & $\begin{array}{c}\text { Mg } \\
\text { (wt \%) }\end{array}$ & $\begin{array}{c}\text { Mn } \\
\text { (wt\%) }\end{array}$ & $\begin{array}{c}\mathbf{S i} \\
\text { (wt\%) }\end{array}$ \\
\hline LRM & 1 & 1 & 1 & LRMPF111 & 5.12 & 2.45 & 0.210 & 0.203 & 1.02 & $<0.100$ & $<0.100$ & $<0.100$ & 25.1 \\
\hline EWG-OL-1580-Q & 1 & 1 & 2 & P11PF11 & 7.92 & 2.45 & 7.19 & 0.952 & $<0.100$ & 2.79 & $<0.100$ & 2.40 & 19.7 \\
\hline RSM-EWG-1-034-Q & 1 & 1 & 3 & P09PF11 & 12.8 & 5.69 & 4.02 & 0.210 & 4.28 & 1.70 & $<0.100$ & $<0.100$ & 13.3 \\
\hline EWG-OL-31644-Q & 1 & 1 & 4 & P07PF21 & 7.93 & 2.52 & 7.45 & 0.997 & 6.95 & 0.640 & 2.26 & 2.41 & 18.9 \\
\hline EWG-OL-2619-Q & 1 & 1 & 5 & P13PF21 & 9.65 & 6.72 & 7.42 & $<0.100$ & 6.92 & $<0.100$ & $<0.100$ & 2.40 & 9.33 \\
\hline EWG-OL-1672-Q & 1 & 1 & 6 & P18PF11 & 13.7 & 9.57 & 6.49 & 0.916 & $<0.100$ & $<0.100$ & $<0.100$ & 2.08 & 8.53 \\
\hline EWG-HAI-Centroid-1-Q & 1 & 1 & 7 & P12PF11 & 11.7 & 4.73 & 2.69 & 0.493 & 3.94 & 1.43 & 0.134 & 0.799 & 14.7 \\
\hline EWG-OL-2619-Q & 1 & 1 & 8 & P13PF11 & 10.0 & 7.02 & 7.31 & $<0.100$ & 7.23 & $<0.100$ & $<0.100$ & 2.51 & 9.69 \\
\hline EWG-OL-15968-Q & 1 & 1 & 9 & P14PF11 & 8.06 & 6.96 & $<0.100$ & 0.956 & $<0.100$ & 2.45 & 2.30 & $<0.100$ & 13.7 \\
\hline EWG-OL-5155-Q & 1 & 1 & 10 & P17PF11 & 10.5 & 7.00 & $<0.100$ & 0.732 & 6.47 & 2.85 & 2.15 & 2.33 & 9.55 \\
\hline EWG-OL-15968-Q & 1 & 1 & 11 & P14PF21 & 7.82 & 6.66 & $<0.100$ & 0.937 & $<0.100$ & 2.40 & 2.25 & $<0.100$ & 13.3 \\
\hline LRM & 1 & 1 & 12 & LRMPF112 & 5.18 & 2.49 & 0.178 & 0.196 & 1.02 & $<0.100$ & $<0.100$ & $<0.100$ & 25.0 \\
\hline EWG-OL-5801-Q & 1 & 1 & 13 & P08PF11 & 9.69 & 6.79 & $<0.100$ & $<0.100$ & 6.85 & 2.79 & 2.22 & 2.40 & 9.23 \\
\hline RSM-EWG-1-034-Q & 1 & 1 & 14 & P09PF21 & 12.5 & 5.77 & 4.13 & 0.212 & 4.16 & 1.69 & $<0.100$ & $<0.100$ & 12.9 \\
\hline EWG-HAI-Centroid-1-Q & 1 & 1 & 15 & P12PF21 & 11.5 & 4.81 & 2.72 & 0.481 & 3.88 & 1.43 & 0.130 & 0.788 & 14.5 \\
\hline EWG-OL-1580-Q & 1 & 1 & 16 & P11PF21 & 7.93 & 2.58 & 7.52 & 0.998 & $<0.100$ & 2.76 & $<0.100$ & 2.41 & 19.4 \\
\hline EWG-HAI-Centroid-2-Q & 1 & 1 & 17 & P10PF21 & 10.6 & 7.32 & 2.30 & 0.413 & 3.58 & 1.31 & 0.101 & 0.728 & 13.6 \\
\hline EWG-OL-1672-Q & 1 & 1 & 18 & P18PF21 & 13.8 & 9.60 & 6.09 & 0.844 & $<0.100$ & $<0.100$ & $<0.100$ & 1.99 & 8.50 \\
\hline EWG-OL-5801-Q & 1 & 1 & 19 & P08PF21 & 9.51 & 6.61 & $<0.100$ & $<0.100$ & 6.70 & 2.74 & 2.13 & 2.35 & 9.14 \\
\hline EWG-OL-5155-Q & 1 & 1 & 20 & P17PF21 & 10.5 & 6.97 & $<0.100$ & 0.747 & 6.52 & 2.84 & 2.19 & 2.35 & 9.49 \\
\hline EWG-HAI-Centroid-2-Q & 1 & 1 & 21 & P10PF11 & 10.6 & 7.34 & 2.38 & 0.420 & 3.57 & 1.32 & 0.106 & 0.727 & 13.5 \\
\hline EWG-OL-31644-Q & 1 & 1 & 22 & P07PF11 & 8.26 & 2.69 & 7.76 & 0.998 & 7.07 & 0.682 & 2.28 & 2.47 & 19.4 \\
\hline LRM & 1 & 1 & 23 & LRMPF113 & 5.22 & 2.53 & 0.198 & 0.198 & 1.04 & $<0.100$ & $<0.100$ & $<0.100$ & 25.3 \\
\hline LRM & 1 & 2 & 1 & LRMPF111 & 5.14 & 2.43 & 0.245 & 0.220 & 0.966 & $<0.100$ & $<0.100$ & $<0.100$ & 25.2 \\
\hline RSM-EWG-1-034-Q & 1 & 2 & 2 & P09PF22 & 12.4 & 5.66 & 4.09 & 0.224 & 4.07 & 1.63 & $<0.100$ & $<0.100$ & 12.9 \\
\hline EWG-HAI-Centroid-1-Q & 1 & 2 & 3 & P12PF12 & 12.0 & 4.78 & 2.71 & 0.506 & 3.99 & 1.43 & 0.149 & 0.747 & 15.2 \\
\hline EWG-HAI-Centroid-1-Q & 1 & 2 & 4 & P12PF22 & 11.6 & 4.73 & 2.66 & 0.482 & 3.85 & 1.41 & 0.141 & 0.725 & 14.7 \\
\hline EWG-OL-1672-Q & 1 & 2 & 5 & P18PF22 & 13.9 & 9.70 & 6.11 & 0.855 & $<0.100$ & $<0.100$ & $<0.100$ & 1.91 & 8.60 \\
\hline EWG-OL-1580-Q & 1 & 2 & 6 & P11PF22 & 8.01 & 2.51 & 7.25 & 0.980 & $<0.100$ & 2.76 & $<0.100$ & 2.38 & 19.8 \\
\hline EWG-OL-5155-Q & 1 & 2 & 7 & P17PF12 & 10.4 & 6.78 & $<0.100$ & 0.727 & 6.38 & 2.76 & 2.10 & 2.25 & 9.57 \\
\hline EWG-OL-5155-Q & 1 & 2 & 8 & P17PF22 & 10.5 & 6.78 & $<0.100$ & 0.722 & 6.43 & 2.75 & 2.08 & 2.27 & 9.55 \\
\hline EWG-OL-15968-Q & 1 & 2 & 9 & P14PF12 & 7.93 & 6.71 & $<0.100$ & 0.945 & $<0.100$ & 2.36 & 2.25 & $<0.100$ & 13.6 \\
\hline EWG-OL-31644-Q & 1 & 2 & 10 & P07PF22 & 7.84 & 2.45 & 7.43 & 0.998 & 6.83 & 0.614 & 2.24 & 2.32 & 18.7 \\
\hline EWG-OL-5801-Q & 1 & 2 & 11 & P08PF12 & 9.71 & 6.75 & $<0.100$ & $<0.100$ & 6.83 & 2.75 & 2.17 & 2.34 & 9.32 \\
\hline LRM & 1 & 2 & 12 & LRMPF122 & 5.21 & 2.41 & 0.235 & 0.216 & 0.975 & $<0.100$ & $<0.100$ & $<0.100$ & 25.5 \\
\hline EWG-HAI-Centroid-2-Q & 1 & 2 & 13 & P10PF22 & 10.4 & 7.10 & 2.32 & 0.428 & 3.47 & 1.25 & 0.120 & 0.645 & 13.5 \\
\hline RSM-EWG-1-034-Q & 1 & 2 & 14 & P09PF12 & 12.9 & 5.74 & 3.99 & 0.222 & 4.25 & 1.68 & $<0.100$ & $<0.100$ & 13.4 \\
\hline EWG-OL-5801-Q & 1 & 2 & 15 & P08PF22 & 9.69 & 6.81 & $<0.100$ & $<0.100$ & 6.80 & 2.76 & 2.12 & 2.33 & 9.45 \\
\hline
\end{tabular}


Table A-3. PF Measurements of Set 1 Study Glasses (continued)

\begin{tabular}{|c|c|c|c|c|c|c|c|c|c|c|c|c|c|}
\hline Glass ID & Block & Sub-Blk & Sequence & Lab ID & $\begin{array}{c}\text { Al } \\
\text { (wt\%) }\end{array}$ & $\begin{array}{c}\text { B } \\
\text { (wt\%) }\end{array}$ & $\begin{array}{c}\text { Ca } \\
\text { (wt\%) }\end{array}$ & $\begin{array}{c}\mathrm{Cr} \\
\text { (wt\%) }\end{array}$ & $\begin{array}{c}\mathbf{F e} \\
(w t \%)\end{array}$ & $\begin{array}{c}\mathbf{L i} \\
\text { (wt\%) }\end{array}$ & $\begin{array}{c}\text { Mg } \\
\text { (wt\%) }\end{array}$ & $\begin{array}{c}\text { Mn } \\
\text { (wt\%) }\end{array}$ & $\begin{array}{c}\mathrm{Si} \\
\text { (wt\%) }\end{array}$ \\
\hline EWG-OL-2619-Q & 1 & 2 & 16 & P13PF22 & 9.77 & 6.72 & 7.01 & $<0.100$ & 6.96 & $<0.100$ & $<0.100$ & 2.37 & 9.44 \\
\hline EWG-HAI-Centroid-2-Q & 1 & 2 & 17 & P10PF12 & 10.7 & 7.39 & 2.37 & 0.432 & 3.56 & 1.30 & 0.122 & 0.668 & 13.7 \\
\hline EWG-OL-31644-Q & 1 & 2 & 18 & P07PF12 & 8.19 & 2.53 & 7.44 & 0.984 & 7.01 & 0.658 & 2.23 & 2.39 & 19.4 \\
\hline EWG-OL-15968-Q & 1 & 2 & 19 & P14PF22 & 7.86 & 6.71 & $<0.100$ & 0.948 & $<0.100$ & 2.34 & 2.26 & $<0.100$ & 13.5 \\
\hline EWG-OL-1580-Q & 1 & 2 & 20 & P11PF12 & 7.92 & 2.50 & 7.27 & 0.963 & $<0.100$ & 2.75 & $<0.100$ & 2.35 & 19.8 \\
\hline EWG-OL-2619-Q & 1 & 2 & 21 & P13PF12 & 9.93 & 6.96 & 7.11 & $<0.100$ & 7.16 & $<0.100$ & $<0.100$ & 2.43 & 9.61 \\
\hline EWG-OL-1672-Q & 1 & 2 & 22 & P18PF12 & 13.9 & 9.72 & 6.21 & 0.889 & $<0.100$ & $<0.100$ & $<0.100$ & 2.05 & 8.65 \\
\hline LRM & 1 & 2 & 23 & LRMPF123 & 5.18 & 2.42 & 0.228 & 0.215 & 0.971 & $<0.100$ & $<0.100$ & $<0.100$ & 25.3 \\
\hline LRM & 2 & 1 & 1 & LRMPF211 & 5.27 & 2.57 & 0.219 & 0.197 & 1.03 & $<0.100$ & $<0.100$ & $<0.100$ & 25.8 \\
\hline EWG-OL-38552-Q & 2 & 1 & 2 & P06PF21 & 14.0 & 2.62 & $<0.100$ & $<0.100$ & 2.61 & 2.85 & 2.22 & 2.46 & 15.8 \\
\hline EWG-OL-29285-Q & 2 & 1 & 3 & P01PF11 & 8.08 & 5.80 & 7.38 & $<0.100$ & $<0.100$ & $<0.100$ & 2.06 & $<0.100$ & 18.4 \\
\hline EWG-OL-26012-Q & 2 & 1 & 4 & P03PF11 & 7.94 & 2.51 & $<0.100$ & $<0.100$ & $<0.100$ & 0.784 & $<0.100$ & $<0.100$ & 20.1 \\
\hline EWG-OL-38552-Q & 2 & 1 & 5 & P06PF11 & 13.5 & 2.47 & $<0.100$ & $<0.100$ & 2.50 & 2.77 & 2.13 & 2.36 & 15.2 \\
\hline EWG-OL-29285-Q & 2 & 1 & 6 & P01PF21 & 7.95 & 5.75 & 7.16 & $<0.100$ & $<0.100$ & $<0.100$ & 2.11 & $<0.100$ & 18.2 \\
\hline EWG-OL-33115-Q & 2 & 1 & 7 & P04PF11 & 8.00 & 2.55 & 7.04 & $<0.100$ & 7.02 & $<0.100$ & 2.22 & $<0.100$ & 16.9 \\
\hline EWG-OL-1369-Q & 2 & 1 & 8 & P19PF11 & 7.93 & 2.48 & 7.08 & 1.02 & $<0.100$ & 2.77 & $<0.100$ & $<0.100$ & 20.0 \\
\hline EWG-OL-6198-Q & 2 & 1 & 9 & P16PF11 & 7.95 & 6.72 & $<0.100$ & 1.05 & 7.00 & 2.80 & 2.15 & $<0.100$ & 11.7 \\
\hline EWG-OL-801-Q & 2 & 1 & 10 & P20PF11 & 16.1 & 6.74 & $<0.100$ & $<0.100$ & $<0.100$ & $<0.100$ & $<0.100$ & 2.43 & 9.58 \\
\hline EWG-OL-1369-Q & 2 & 1 & 11 & P19PF21 & 8.01 & 2.55 & 6.96 & 1.03 & $<0.100$ & 2.84 & $<0.100$ & $<0.100$ & 20.2 \\
\hline LRM & 2 & 1 & 12 & LRMPF212 & 5.22 & 2.44 & 0.227 & 0.198 & 1.01 & $<0.100$ & $<0.100$ & $<0.100$ & 25.5 \\
\hline EWG-OL-2463-Q & 2 & 1 & 13 & P15PF11 & 10.2 & 2.47 & 6.80 & $<0.100$ & 6.87 & $<0.100$ & $<0.100$ & 2.37 & 9.41 \\
\hline EWG-OL-14827-Q & 2 & 1 & 14 & P05PF11 & 8.18 & 6.90 & 7.14 & 1.07 & $<0.100$ & 2.82 & $<0.100$ & 2.48 & 14.5 \\
\hline EWG-OL-801-Q & 2 & 1 & 15 & P20PF21 & 15.5 & 6.51 & $<0.100$ & $<0.100$ & $<0.100$ & $<0.100$ & $<0.100$ & 2.33 & 9.14 \\
\hline EWG-OL-3872-Q & 2 & 1 & 16 & P02PF11 & 7.90 & 2.50 & 6.92 & $<0.100$ & $<0.100$ & 2.78 & 2.20 & $<0.100$ & 14.6 \\
\hline EWG-OL-3872-Q & 2 & 1 & 17 & P02PF21 & 7.72 & 2.47 & 7.02 & $<0.100$ & $<0.100$ & 2.72 & 2.22 & $<0.100$ & 14.3 \\
\hline EWG-OL-26012-Q & 2 & 1 & 18 & P03PF21 & 7.93 & 2.44 & $<0.100$ & $<0.100$ & $<0.100$ & 0.780 & $<0.100$ & $<0.100$ & 19.8 \\
\hline EWG-OL-2463-Q & 2 & 1 & 19 & P15PF21 & 10.3 & 2.50 & 6.91 & $<0.100$ & 6.97 & $<0.100$ & $<0.100$ & 2.40 & 9.35 \\
\hline EWG-OL-33115-Q & 2 & 1 & 20 & P04PF21 & 8.14 & 2.59 & 6.97 & $<0.100$ & 7.15 & $<0.100$ & 2.20 & $<0.100$ & 17.1 \\
\hline EWG-OL-6198-Q & 2 & 1 & 21 & P16PF21 & 8.01 & 6.83 & $<0.100$ & 1.02 & 7.02 & 2.83 & 2.10 & $<0.100$ & 11.7 \\
\hline EWG-OL-14827-Q & 2 & 1 & 22 & P05PF21 & 7.92 & 6.77 & 6.76 & 1.01 & $<0.100$ & 2.74 & $<0.100$ & 2.39 & 14.1 \\
\hline LRM & 2 & 1 & 23 & LRMPF213 & 5.21 & 2.43 & 0.241 & 0.201 & 1.01 & $<0.100$ & $<0.100$ & $<0.100$ & 25.2 \\
\hline LRM & 2 & 2 & 1 & LRMPF221 & 5.06 & 2.45 & 0.216 & 0.200 & 0.961 & $<0.100$ & $<0.100$ & $<0.100$ & 24.8 \\
\hline EWG-OL-29285-Q & 2 & 2 & 2 & P01PF22 & 7.86 & 5.66 & 7.12 & $<0.100$ & $<0.100$ & $<0.100$ & 2.11 & $<0.100$ & 18.0 \\
\hline EWG-OL-3872-Q & 2 & 2 & 3 & P02PF12 & 7.77 & 2.52 & 7.04 & $<0.100$ & $<0.100$ & 2.67 & 2.23 & $<0.100$ & 14.5 \\
\hline EWG-OL-38552-Q & 2 & 2 & 4 & P06PF22 & 13.8 & 2.58 & $<0.100$ & $<0.100$ & 2.55 & 2.75 & 2.25 & 2.39 & 15.6 \\
\hline EWG-OL-6198-Q & 2 & 2 & 5 & P16PF12 & 8.03 & 6.95 & $<0.100$ & 1.07 & 7.08 & 2.77 & 2.18 & $<0.100$ & 11.8 \\
\hline EWG-OL-14827-Q & 2 & 2 & 6 & P05PF22 & 7.92 & 6.92 & 6.90 & 1.02 & $<0.100$ & 2.68 & $<0.100$ & 2.36 & 14.2 \\
\hline EWG-OL-38552-Q & 2 & 2 & 7 & P06PF12 & 13.7 & 2.58 & $<0.100$ & $<0.100$ & 2.51 & 2.74 & 2.18 & 2.35 & 15.4 \\
\hline
\end{tabular}


Table A-3. PF Measurements of Set 1 Study Glasses (continued)

\begin{tabular}{|c|c|c|c|c|c|c|c|c|c|c|c|c|c|}
\hline Glass ID & Block & Sub-Blk & Sequence & Lab ID & $\begin{array}{c}\text { Al } \\
\text { (wt\%) }\end{array}$ & $\begin{array}{c}\text { B } \\
\text { (wt\%) }\end{array}$ & $\begin{array}{c}\mathrm{Ca} \\
\text { (wt\%) }\end{array}$ & $\begin{array}{c}\mathrm{Cr} \\
\text { (wt\%) }\end{array}$ & $\begin{array}{c}\mathbf{F e} \\
\text { (wt\%) }\end{array}$ & $\begin{array}{c}\mathbf{L i} \\
\text { (wt\%) }\end{array}$ & $\begin{array}{c}\text { Mg } \\
\text { (wt\%) }\end{array}$ & $\begin{array}{c}\text { Mn } \\
\text { (wt\%) }\end{array}$ & $\begin{array}{c}\mathrm{Si} \\
\text { (wt\%) }\end{array}$ \\
\hline EWG-OL-2463-Q & 2 & 2 & 8 & P15PF12 & 10.4 & 2.58 & 7.02 & $<0.100$ & 6.99 & $<0.100$ & $<0.100$ & 2.37 & 9.57 \\
\hline EWG-OL-33115-Q & 2 & 2 & 9 & P04PF12 & 7.97 & 2.57 & 6.88 & $<0.100$ & 6.96 & $<0.100$ & 2.16 & $<0.100$ & 16.8 \\
\hline EWG-OL-14827-Q & 2 & 2 & 10 & P05PF12 & 8.17 & 6.99 & 6.88 & 1.03 & $<0.100$ & 2.75 & $<0.100$ & 2.44 & 14.5 \\
\hline EWG-OL-801-Q & 2 & 2 & 11 & P20PF22 & 15.2 & 6.29 & $<0.100$ & $<0.100$ & $<0.100$ & $<0.100$ & $<0.100$ & 2.25 & 8.95 \\
\hline LRM & 2 & 2 & 12 & LRMPF222 & 5.07 & 2.40 & 0.236 & 0.207 & 0.954 & $<0.100$ & $<0.100$ & $<0.100$ & 24.7 \\
\hline EWG-OL-1369-Q & 2 & 2 & 13 & P19PF22 & 7.87 & 2.58 & 7.11 & 1.04 & $<0.100$ & 2.74 & $<0.100$ & $<0.100$ & 19.9 \\
\hline EWG-OL-1369-Q & 2 & 2 & 14 & P19PF12 & 8.00 & 2.55 & 7.23 & 1.03 & $<0.100$ & 2.73 & $<0.100$ & $<0.100$ & 20.1 \\
\hline EWG-OL-6198-Q & 2 & 2 & 15 & P16PF22 & 7.90 & 6.73 & $<0.100$ & 1.04 & 6.93 & 2.74 & 2.12 & $<0.100$ & 11.6 \\
\hline EWG-OL-26012-Q & 2 & 2 & 16 & P03PF12 & 7.93 & 2.53 & $<0.100$ & $<0.100$ & $<0.100$ & 0.780 & $<0.100$ & $<0.100$ & 20.0 \\
\hline EWG-OL-801-Q & 2 & 2 & 17 & P20PF12 & 15.7 & 6.54 & $<0.100$ & $<0.100$ & $<0.100$ & $<0.100$ & $<0.100$ & 2.33 & 9.24 \\
\hline EWG-OL-26012-Q & 2 & 2 & 18 & P03PF22 & 7.85 & 2.47 & $<0.100$ & $<0.100$ & $<0.100$ & 0.767 & $<0.100$ & $<0.100$ & 19.7 \\
\hline EWG-OL-3872-Q & 2 & 2 & 19 & P02PF22 & 7.82 & 2.57 & 7.21 & $<0.100$ & $<0.100$ & 2.73 & 2.25 & $<0.100$ & 14.6 \\
\hline EWG-OL-2463-Q & 2 & 2 & 20 & P15PF22 & 10.3 & 2.55 & 7.16 & $<0.100$ & 6.95 & $<0.100$ & $<0.100$ & 2.36 & 9.32 \\
\hline EWG-OL-33115-Q & 2 & 2 & 21 & P04PF22 & 8.00 & 2.55 & 7.07 & $<0.100$ & 7.04 & $<0.100$ & 2.22 & $<0.100$ & 16.9 \\
\hline EWG-OL-29285-Q & 2 & 2 & 22 & P01PF12 & 7.98 & 5.70 & 7.52 & $<0.100$ & $<0.100$ & $<0.100$ & 2.10 & $<0.100$ & 18.1 \\
\hline LRM & 2 & 2 & 23 & LRMPF223 & 5.15 & 2.37 & 0.235 & 0.204 & 0.979 & $<0.100$ & $<0.100$ & $<0.100$ & 25.0 \\
\hline
\end{tabular}


Table A-4. Comparison of Measured and Targeted Compositions for Set 1 Study Glasses

\begin{tabular}{|c|c|c|c|c|c|}
\hline Glass ID & Oxide & $\begin{array}{l}\text { Measured } \\
\text { (wt \%) }\end{array}$ & $\begin{array}{c}\text { Targeted } \\
\text { (wt \%) }\end{array}$ & $\begin{array}{l}\text { Difference of Measured } \\
\text { versus Targeted }\end{array}$ & $\begin{array}{c}\text { \% Difference of Measured } \\
\text { versus Targeted }\end{array}$ \\
\hline EWG-HAI-Centroid-1-Q & $\mathrm{Al}_{2} \mathrm{O}_{3}$ & 22.1072 & 22.0000 & 0.1072 & $0.5 \%$ \\
\hline EWG-HAI-Centroid-1-Q & $\mathrm{B}_{2} \mathrm{O}_{3}$ & 15.3348 & 15.5000 & -0.1652 & $-1.1 \%$ \\
\hline EWG-HAI-Centroid-1-Q & $\mathrm{Bi}_{2} \mathrm{O}_{3}$ & 0.9752 & 1.0000 & -0.0248 & $-2.5 \%$ \\
\hline EWG-HAI-Centroid-1-Q & $\mathrm{CaO}$ & 3.7708 & 3.5000 & 0.2708 & $7.7 \%$ \\
\hline EWG-HAI-Centroid-1-Q & $\mathrm{CdO}$ & 0.0865 & 0.1000 & -0.0135 & $-13.5 \%$ \\
\hline EWG-HAI-Centroid-1-Q & $\mathrm{Cr}_{2} \mathrm{O}_{3}$ & 0.7169 & 0.7500 & -0.0331 & $-4.4 \%$ \\
\hline EWG-HAI-Centroid-1-Q & $\mathrm{Fe}_{2} \mathrm{O}_{3}$ & 5.5973 & 5.5000 & 0.0973 & $1.8 \%$ \\
\hline EWG-HAI-Centroid-1-Q & $\mathrm{K}_{2} \mathrm{O}$ & 0.6815 & 0.7000 & -0.0185 & $-2.6 \%$ \\
\hline EWG-HAI-Centroid-1-Q & $\mathrm{Li}_{2} \mathrm{O}$ & 3.0679 & 3.0000 & 0.0679 & $2.3 \%$ \\
\hline EWG-HAI-Centroid-1-Q & $\mathrm{MgO}$ & 0.2297 & 0.5000 & -0.2703 & $-54.1 \%$ \\
\hline EWG-HAI-Centroid-1-Q & $\mathrm{MnO}$ & 0.9874 & 1.0000 & -0.0126 & $-1.3 \%$ \\
\hline EWG-HAI-Centroid-1-Q & $\mathrm{Na}_{2} \mathrm{O}$ & 11.8894 & 11.5000 & 0.3894 & $3.4 \%$ \\
\hline EWG-HAI-Centroid-1-Q & $\mathrm{NiO}$ & 0.1743 & 0.4000 & -0.2257 & $-56.4 \%$ \\
\hline EWG-HAI-Centroid-1-Q & $\mathrm{P}_{2} \mathrm{O}_{5}$ & 0.4680 & 0.5000 & -0.0320 & $-6.4 \%$ \\
\hline EWG-HAI-Centroid-1-Q & $\mathrm{PbO}$ & 0.2704 & 0.3000 & -0.0296 & $-9.9 \%$ \\
\hline EWG-HAI-Centroid-1-Q & $\mathrm{RuO}_{2}$ & 0.0132 & 0.0100 & 0.0032 & $31.7 \%$ \\
\hline EWG-HAI-Centroid-1-Q & $\mathrm{SiO}_{2}$ & 31.6082 & 31.5000 & 0.1082 & $0.3 \%$ \\
\hline EWG-HAI-Centroid-1-Q & $\mathrm{SO}_{3}$ & 0.2434 & 0.3000 & -0.0566 & $-18.9 \%$ \\
\hline EWG-HAI-Centroid-1-Q & $\mathrm{SrO}$ & 0.1168 & 0.1200 & -0.0032 & $-2.7 \%$ \\
\hline EWG-HAI-Centroid-1-Q & $\mathrm{ZrO}_{2}$ & 0.9098 & 1.0000 & -0.0902 & $-9.0 \%$ \\
\hline EWG-HAI-Centroid-1-Q & Sum & 99.2486 & 99.1800 & 0.0686 & $0.1 \%$ \\
\hline EWG-HAI-Centroid-2-Q & $\mathrm{Al}_{2} \mathrm{O}_{3}$ & 19.9815 & 22.0000 & -2.0185 & $-9.2 \%$ \\
\hline EWG-HAI-Centroid-2-Q & $\mathrm{B}_{2} \mathrm{O}_{3}$ & 23.4650 & 15.5000 & 7.9650 & $51.4 \%$ \\
\hline EWG-HAI-Centroid-2-Q & $\mathrm{Bi}_{2} \mathrm{O}_{3}$ & 0.8826 & 1.0000 & -0.1174 & $-11.7 \%$ \\
\hline EWG-HAI-Centroid-2-Q & $\mathrm{CaO}$ & 3.2776 & 3.5000 & -0.2224 & $-6.4 \%$ \\
\hline EWG-HAI-Centroid-2-Q & $\mathrm{CdO}$ & 0.0780 & 0.1000 & -0.0220 & $-22.0 \%$ \\
\hline EWG-HAI-Centroid-2-Q & $\mathrm{Cr}_{2} \mathrm{O}_{3}$ & 0.6186 & 0.7500 & -0.1314 & $-17.5 \%$ \\
\hline EWG-HAI-Centroid-2-Q & $\mathrm{Fe}_{2} \mathrm{O}_{3}$ & 5.0683 & 5.5000 & -0.4317 & $-7.8 \%$ \\
\hline EWG-HAI-Centroid-2-Q & $\mathrm{K}_{2} \mathrm{O}$ & 0.4879 & 0.7000 & -0.2121 & $-30.3 \%$ \\
\hline EWG-HAI-Centroid-2-Q & $\mathrm{Li}_{2} \mathrm{O}$ & 2.7880 & 3.0000 & -0.2120 & $-7.1 \%$ \\
\hline EWG-HAI-Centroid-2-Q & $\mathrm{MgO}$ & 0.1861 & 0.5000 & -0.3139 & $-62.8 \%$ \\
\hline EWG-HAI-Centroid-2-Q & $\mathrm{MnO}$ & 0.8935 & 1.0000 & -0.1065 & $-10.6 \%$ \\
\hline EWG-HAI-Centroid-2-Q & $\mathrm{Na}_{2} \mathrm{O}$ & 10.5313 & 11.5000 & -0.9688 & $-8.4 \%$ \\
\hline EWG-HAI-Centroid-2-Q & $\mathrm{NiO}$ & 0.1492 & 0.4000 & -0.2508 & $-62.7 \%$ \\
\hline EWG-HAI-Centroid-2-Q & $\mathrm{P}_{2} \mathrm{O}_{5}$ & 0.8249 & 1.0000 & -0.1751 & $-17.5 \%$ \\
\hline EWG-HAI-Centroid-2-Q & $\mathrm{PbO}$ & 0.2429 & 0.3000 & -0.0571 & $-19.0 \%$ \\
\hline EWG-HAI-Centroid-2-Q & $\mathrm{RuO}_{2}$ & 0.0132 & 0.0100 & 0.0032 & $31.7 \%$ \\
\hline EWG-HAI-Centroid-2-Q & $\mathrm{SiO}_{2}$ & 29.0410 & 31.5000 & -2.4590 & $-7.8 \%$ \\
\hline EWG-HAI-Centroid-2-Q & $\mathrm{SO}_{3}$ & 0.2422 & 0.3000 & -0.0578 & $-19.3 \%$ \\
\hline EWG-HAI-Centroid-2-Q & SrO & 0.1067 & 0.1200 & -0.0133 & $-11.1 \%$ \\
\hline EWG-HAI-Centroid-2-Q & $\mathrm{ZrO}_{2}$ & 0.8388 & 1.0000 & -0.1612 & $-16.1 \%$ \\
\hline EWG-HAI-Centroid-2-Q & Sum & 99.7173 & 99.6800 & 0.0373 & $0.0 \%$ \\
\hline EWG-OL-1369-Q & $\mathrm{Al}_{2} \mathrm{O}_{3}$ & 15.0262 & 15.0000 & 0.0262 & $0.2 \%$ \\
\hline EWG-OL-1369-Q & $\mathrm{B}_{2} \mathrm{O}_{3}$ & 8.1785 & 8.0000 & 0.1785 & $2.2 \%$ \\
\hline EWG-OL-1369-Q & $\mathrm{Bi}_{2} \mathrm{O}_{3}$ & 2.9626 & 3.0000 & -0.0374 & $-1.2 \%$ \\
\hline EWG-OL-1369-Q & $\mathrm{CaO}$ & 9.9273 & 10.0000 & -0.0727 & $-0.7 \%$ \\
\hline EWG-OL-1369-Q & $\mathrm{CdO}$ & 0.0848 & 0.1000 & -0.0152 & $-15.2 \%$ \\
\hline EWG-OL-1369-Q & $\mathrm{Cr}_{2} \mathrm{O}_{3}$ & 1.5054 & 1.6000 & -0.0946 & $-5.9 \%$ \\
\hline EWG-OL-1369-Q & $\mathrm{Fe}_{2} \mathrm{O}_{3}$ & 0.1430 & 0.0000 & 0.1430 & \\
\hline EWG-OL-1369-Q & $\mathrm{K}_{2} \mathrm{O}$ & 0.0120 & 0.0000 & 0.0120 & \\
\hline EWG-OL-1369-Q & $\mathrm{Li}_{2} \mathrm{O}$ & 5.9635 & 6.0000 & -0.0365 & $-0.6 \%$ \\
\hline EWG-OL-1369-Q & $\mathrm{MgO}$ & 0.1658 & 0.0000 & 0.1658 & \\
\hline EWG-OL-1369-Q & $\mathrm{MnO}$ & 0.1291 & 0.0000 & 0.1291 & \\
\hline EWG-OL-1369-Q & $\mathrm{Na}_{2} \mathrm{O}$ & 5.0011 & 5.0000 & 0.0011 & $0.0 \%$ \\
\hline EWG-OL-1369-Q & $\mathrm{NiO}$ & 0.3690 & 0.4000 & -0.0310 & $-7.7 \%$ \\
\hline EWG-OL-1369-Q & $\mathrm{P}_{2} \mathrm{O}_{5}$ & 2.7783 & 3.0000 & -0.2217 & $-7.4 \%$ \\
\hline EWG-OL-1369-Q & $\mathrm{PbO}$ & 0.2650 & 0.3000 & -0.0350 & $-11.7 \%$ \\
\hline EWG-OL-1369-Q & $\mathrm{RuO}_{2}$ & 0.0132 & 0.0100 & 0.0032 & $31.7 \%$ \\
\hline EWG-OL-1369-Q & $\mathrm{SiO}_{2}$ & 42.8930 & 42.8550 & 0.0380 & $0.1 \%$ \\
\hline EWG-OL-1369-Q & $\mathrm{SO}_{3}$ & 0.2466 & 0.3000 & -0.0534 & $-17.8 \%$ \\
\hline
\end{tabular}


Table A-4. Comparison of Measured and Targeted Compositions for Set 1 Study Glasses (continued)

\begin{tabular}{|c|c|c|c|c|c|}
\hline Glass ID & Oxide & $\begin{array}{l}\text { Measured } \\
\text { (wt \%) }\end{array}$ & $\begin{array}{c}\text { Targeted } \\
\text { (wt \%) }\end{array}$ & $\begin{array}{c}\text { Difference of Measured } \\
\text { versus Targeted }\end{array}$ & $\begin{array}{c}\text { \% Difference of Measured } \\
\text { versus Targeted }\end{array}$ \\
\hline EWG-OL-1369-Q & $\mathrm{SrO}$ & 0.1094 & 0.1200 & -0.0106 & $-8.8 \%$ \\
\hline EWG-OL-1369-Q & $\mathrm{ZrO}_{2}$ & 3.9680 & 4.0000 & -0.0320 & $-0.8 \%$ \\
\hline EWG-OL-1369-Q & Sum & 99.7419 & 99.6850 & 0.0569 & $0.1 \%$ \\
\hline EWG-OL-14827-Q & $\mathrm{Al}_{2} \mathrm{O}_{3}$ & 15.2058 & 15.0000 & 0.2058 & $1.4 \%$ \\
\hline EWG-OL-14827-Q & $\mathrm{B}_{2} \mathrm{O}_{3}$ & 22.2012 & 22.0000 & 0.2012 & $0.9 \%$ \\
\hline EWG-OL-14827-Q & $\mathrm{Bi}_{2} \mathrm{O}_{3}$ & 2.9598 & 3.0000 & -0.0402 & $-1.3 \%$ \\
\hline EWG-OL-14827-Q & $\mathrm{CaO}$ & 9.6825 & 10.0000 & -0.3175 & $-3.2 \%$ \\
\hline EWG-OL-14827-Q & $\mathrm{CdO}$ & 0.0817 & 0.1000 & -0.0183 & $-18.3 \%$ \\
\hline EWG-OL-14827-Q & $\mathrm{Cr}_{2} \mathrm{O}_{3}$ & 1.5091 & 1.6000 & -0.0909 & $-5.7 \%$ \\
\hline EWG-OL-14827-Q & $\mathrm{Fe}_{2} \mathrm{O}_{3}$ & 0.1430 & 0.0000 & 0.1430 & \\
\hline EWG-OL-14827-Q & $\mathrm{K}_{2} \mathrm{O}$ & 3.1771 & 3.0000 & 0.1771 & $5.9 \%$ \\
\hline EWG-OL-14827-Q & $\mathrm{Li}_{2} \mathrm{O}$ & 5.9151 & 5.8994 & 0.0157 & $0.3 \%$ \\
\hline EWG-OL-14827-Q & $\mathrm{MgO}$ & 0.1658 & 0.0000 & 0.1658 & \\
\hline EWG-OL-14827-Q & $\mathrm{MnO}$ & 3.1215 & 3.0000 & 0.1215 & $4.0 \%$ \\
\hline EWG-OL-14827-Q & $\mathrm{Na}_{2} \mathrm{O}$ & 5.0786 & 5.0000 & 0.0786 & $1.6 \%$ \\
\hline EWG-OL-14827-Q & $\mathrm{NiO}$ & 0.2879 & 0.4000 & -0.1121 & $-28.0 \%$ \\
\hline EWG-OL-14827-Q & $\mathrm{P}_{2} \mathrm{O}_{5}$ & 0.2291 & 0.0000 & 0.2291 & \\
\hline EWG-OL-14827-Q & $\mathrm{PbO}$ & 0.2658 & 0.3000 & -0.0342 & $-11.4 \%$ \\
\hline EWG-OL-14827-Q & $\mathrm{RuO}_{2}$ & 0.0132 & 0.0100 & 0.0032 & $31.7 \%$ \\
\hline EWG-OL-14827-Q & $\mathrm{SiO}_{2}$ & 30.6455 & 29.9556 & 0.6899 & $2.3 \%$ \\
\hline EWG-OL-14827-Q & $\mathrm{SO}_{3}$ & 0.3115 & 0.3000 & 0.0115 & $3.8 \%$ \\
\hline EWG-OL-14827-Q & $\mathrm{SrO}$ & 0.1144 & 0.1200 & -0.0056 & $-4.7 \%$ \\
\hline EWG-OL-14827-Q & $\mathrm{ZrO}_{2}$ & 0.1351 & 0.0000 & 0.1351 & \\
\hline EWG-OL-14827-Q & Sum & 101.2436 & 99.6850 & 1.5586 & $1.6 \%$ \\
\hline EWG-OL-1580-Q & $\mathrm{Al}_{2} \mathrm{O}_{3}$ & 15.0121 & 15.0000 & 0.0121 & $0.1 \%$ \\
\hline EWG-OL-1580-Q & $\mathrm{B}_{2} \mathrm{O}_{3}$ & 8.0819 & 8.0000 & 0.0819 & $1.0 \%$ \\
\hline EWG-OL-1580-Q & $\mathrm{Bi}_{2} \mathrm{O}_{3}$ & 0.1115 & 0.0000 & 0.1115 & \\
\hline EWG-OL-1580-Q & $\mathrm{CaO}$ & 10.2247 & 10.0000 & 0.2247 & $2.2 \%$ \\
\hline EWG-OL-1580-Q & $\mathrm{CdO}$ & 0.0862 & 0.1000 & -0.0138 & $-13.8 \%$ \\
\hline EWG-OL-1580-Q & $\mathrm{Cr}_{2} \mathrm{O}_{3}$ & 1.4225 & 1.6000 & -0.1775 & $-11.1 \%$ \\
\hline EWG-OL-1580-Q & $\mathrm{Fe}_{2} \mathrm{O}_{3}$ & 0.1430 & 0.0000 & 0.1430 & \\
\hline EWG-OL-1580-Q & $\mathrm{K}_{2} \mathrm{O}$ & 0.0120 & 0.0000 & 0.0120 & \\
\hline EWG-OL-1580-Q & $\mathrm{Li}_{2} \mathrm{O}$ & 5.9528 & 6.0000 & -0.0472 & $-0.8 \%$ \\
\hline EWG-OL-1580-Q & $\mathrm{MgO}$ & 0.1658 & 0.0000 & 0.1658 & \\
\hline EWG-OL-1580-Q & $\mathrm{MnO}$ & 3.0795 & 3.0000 & 0.0795 & $2.7 \%$ \\
\hline EWG-OL-1580-Q & $\mathrm{Na}_{2} \mathrm{O}$ & 5.1662 & 5.0000 & 0.1662 & $3.3 \%$ \\
\hline EWG-OL-1580-Q & $\mathrm{NiO}$ & 0.3722 & 0.4000 & -0.0278 & $-6.9 \%$ \\
\hline EWG-OL-1580-Q & $\mathrm{P}_{2} \mathrm{O}_{5}$ & 2.7841 & 3.0000 & -0.2159 & $-7.2 \%$ \\
\hline EWG-OL-1580-Q & $\mathrm{PbO}$ & 0.2701 & 0.3000 & -0.0299 & $-10.0 \%$ \\
\hline EWG-OL-1580-Q & $\mathrm{RuO}_{2}$ & 0.0132 & 0.0100 & 0.0032 & $31.7 \%$ \\
\hline EWG-OL-1580-Q & $\mathrm{SiO}_{2}$ & 42.0907 & 42.8550 & -0.7643 & $-1.8 \%$ \\
\hline EWG-OL-1580-Q & $\mathrm{SO}_{3}$ & 0.2797 & 0.3000 & -0.0203 & $-6.8 \%$ \\
\hline EWG-OL-1580-Q & $\mathrm{SrO}$ & 0.1047 & 0.1200 & -0.0153 & $-12.8 \%$ \\
\hline EWG-OL-1580-Q & $\mathrm{ZrO}_{2}$ & 3.9545 & 4.0000 & -0.0455 & $-1.1 \%$ \\
\hline EWG-OL-1580-Q & Sum & 99.3273 & 99.6850 & -0.3577 & $-0.4 \%$ \\
\hline EWG-OL-15968-Q & $\mathrm{Al}_{2} \mathrm{O}_{3}$ & 14.9601 & 15.0000 & -0.0399 & $-0.3 \%$ \\
\hline EWG-OL-15968-Q & $\mathrm{B}_{2} \mathrm{O}_{3}$ & 21.7665 & 22.0000 & -0.2335 & $-1.1 \%$ \\
\hline EWG-OL-15968-Q & $\mathrm{Bi}_{2} \mathrm{O}_{3}$ & 0.1115 & 0.0000 & 0.1115 & \\
\hline EWG-OL-15968-Q & $\mathrm{CaO}$ & 0.1399 & 0.0000 & 0.1399 & \\
\hline EWG-OL-15968-Q & $\mathrm{CdO}$ & 0.0837 & 0.1000 & -0.0163 & $-16.3 \%$ \\
\hline EWG-OL-15968-Q & $\mathrm{Cr}_{2} \mathrm{O}_{3}$ & 1.3834 & 1.6000 & -0.2166 & $-13.5 \%$ \\
\hline EWG-OL-15968-Q & $\mathrm{Fe}_{2} \mathrm{O}_{3}$ & 0.1430 & 0.0000 & 0.1430 & \\
\hline EWG-OL-15968-Q & $\mathrm{K}_{2} \mathrm{O}$ & 0.0120 & 0.0000 & 0.0120 & \\
\hline EWG-OL-15968-Q & $\mathrm{Li}_{2} \mathrm{O}$ & 5.1400 & 5.1490 & -0.0090 & $-0.2 \%$ \\
\hline EWG-OL-15968-Q & $\mathrm{MgO}$ & 3.7560 & 4.0000 & -0.2440 & $-6.1 \%$ \\
\hline EWG-OL-15968-Q & $\mathrm{MnO}$ & 0.1291 & 0.0000 & 0.1291 & \\
\hline EWG-OL-15968-Q & $\mathrm{Na}_{2} \mathrm{O}$ & 18.5350 & 18.0000 & 0.5350 & $3.0 \%$ \\
\hline EWG-OL-15968-Q & $\mathrm{NiO}$ & 0.3226 & 0.4000 & -0.0774 & $-19.4 \%$ \\
\hline EWG-OL-15968-Q & $\mathrm{P}_{2} \mathrm{O}_{5}$ & 0.2291 & 0.0000 & 0.2291 & \\
\hline
\end{tabular}


Table A-4. Comparison of Measured and Targeted Compositions for Set 1 Study Glasses (continued)

\begin{tabular}{|c|c|c|c|c|c|}
\hline Glass ID & Oxide & $\begin{array}{l}\text { Measured } \\
\text { (wt \%) }\end{array}$ & $\begin{array}{l}\text { Targeted } \\
\text { (wt \%) }\end{array}$ & $\begin{array}{c}\text { Difference of Measured } \\
\text { versus Targeted }\end{array}$ & $\begin{array}{c}\text { \% Difference of Measured } \\
\text { versus Targeted }\end{array}$ \\
\hline EWG-OL-15968-Q & $\mathrm{PbO}$ & 0.2715 & 0.3000 & -0.0285 & $-9.5 \%$ \\
\hline EWG-OL-15968-Q & $\mathrm{RuO}_{2}$ & 0.0132 & 0.0100 & 0.0032 & $31.7 \%$ \\
\hline EWG-OL-15968-Q & $\mathrm{SiO}_{2}$ & 28.9340 & 28.7060 & 0.2280 & $0.8 \%$ \\
\hline EWG-OL-15968-Q & $\mathrm{SO}_{3}$ & 0.3015 & 0.3000 & 0.0015 & $0.5 \%$ \\
\hline EWG-OL-15968-Q & $\mathrm{SrO}$ & 0.1162 & 0.1200 & -0.0038 & $-3.2 \%$ \\
\hline EWG-OL-15968-Q & $\mathrm{ZrO}_{2}$ & 3.9646 & 4.0000 & -0.0354 & $-0.9 \%$ \\
\hline EWG-OL-15968-Q & Sum & 100.3130 & 99.6850 & 0.6280 & $0.6 \%$ \\
\hline EWG-OL-1672-Q & $\mathrm{Al}_{2} \mathrm{O}_{3}$ & 26.1223 & 30.0000 & -3.8777 & $-12.9 \%$ \\
\hline EWG-OL-1672-Q & $\mathrm{B}_{2} \mathrm{O}_{3}$ & 31.0640 & 22.0000 & 9.0640 & $41.2 \%$ \\
\hline EWG-OL-1672-Q & $\mathrm{Bi}_{2} \mathrm{O}_{3}$ & 2.7313 & 3.0000 & -0.2687 & $-9.0 \%$ \\
\hline EWG-OL-1672-Q & $\mathrm{CaO}$ & 8.7100 & 10.0000 & -1.2900 & $-12.9 \%$ \\
\hline EWG-OL-1672-Q & $\mathrm{CdO}$ & 0.0754 & 0.1000 & -0.0246 & $-24.6 \%$ \\
\hline EWG-OL-1672-Q & $\mathrm{Cr}_{2} \mathrm{O}_{3}$ & 1.2804 & 1.6000 & -0.3196 & $-20.0 \%$ \\
\hline EWG-OL-1672-Q & $\mathrm{Fe}_{2} \mathrm{O}_{3}$ & 0.1430 & 0.0000 & 0.1430 & \\
\hline EWG-OL-1672-Q & $\mathrm{K}_{2} \mathrm{O}$ & 0.0120 & 0.0000 & 0.0120 & \\
\hline EWG-OL-1672-Q & $\mathrm{Li}_{2} \mathrm{O}$ & 0.2153 & 0.0000 & 0.2153 & \\
\hline EWG-OL-1672-Q & $\mathrm{MgO}$ & 0.1658 & 0.0000 & 0.1658 & \\
\hline EWG-OL-1672-Q & $\mathrm{MnO}$ & 2.5921 & 3.0000 & -0.4079 & $-13.6 \%$ \\
\hline EWG-OL-1672-Q & $\mathrm{Na}_{2} \mathrm{O}$ & 4.5293 & 5.0000 & -0.4707 & $-9.4 \%$ \\
\hline EWG-OL-1672-Q & $\mathrm{NiO}$ & 0.3274 & 0.4000 & -0.0726 & $-18.2 \%$ \\
\hline EWG-OL-1672-Q & $\mathrm{P}_{2} \mathrm{O}_{5}$ & 2.4117 & 3.0000 & -0.5883 & $-19.6 \%$ \\
\hline EWG-OL-1672-Q & $\mathrm{PbO}$ & 0.2424 & 0.3000 & -0.0576 & $-19.2 \%$ \\
\hline EWG-OL-1672-Q & $\mathrm{RuO}_{2}$ & 0.0132 & 0.0100 & 0.0032 & $31.7 \%$ \\
\hline EWG-OL-1672-Q & $\mathrm{SiO}_{2}$ & 18.3338 & 20.8550 & -2.5212 & $-12.1 \%$ \\
\hline EWG-OL-1672-Q & $\mathrm{SO}_{3}$ & 0.0262 & 0.3000 & -0.2738 & $-91.3 \%$ \\
\hline EWG-OL-1672-Q & $\mathrm{SrO}$ & 0.1079 & 0.1200 & -0.0121 & $-10.1 \%$ \\
\hline EWG-OL-1672-Q & $\mathrm{ZrO}_{2}$ & 0.1351 & 0.0000 & 0.1351 & \\
\hline EWG-OL-1672-Q & Sum & 99.2385 & 99.6850 & -0.4465 & $-0.4 \%$ \\
\hline EWG-OL-2463-Q & $\mathrm{Al}_{2} \mathrm{O}_{3}$ & 19.4619 & 19.4550 & 0.0069 & $0.0 \%$ \\
\hline EWG-OL-2463-Q & $\mathrm{B}_{2} \mathrm{O}_{3}$ & 8.1302 & 8.0000 & 0.1302 & $1.6 \%$ \\
\hline EWG-OL-2463-Q & $\mathrm{Bi}_{2} \mathrm{O}_{3}$ & 0.1115 & 0.0000 & 0.1115 & \\
\hline EWG-OL-2463-Q & $\mathrm{CaO}$ & 9.7559 & 10.0000 & -0.2441 & $-2.4 \%$ \\
\hline EWG-OL-2463-Q & $\mathrm{CdO}$ & 0.0831 & 0.1000 & -0.0169 & $-16.9 \%$ \\
\hline EWG-OL-2463-Q & $\mathrm{Cr}_{2} \mathrm{O}_{3}$ & 0.1462 & 0.0000 & 0.1462 & \\
\hline EWG-OL-2463-Q & $\mathrm{Fe}_{2} \mathrm{O}_{3}$ & 9.9293 & 10.0000 & -0.0707 & $-0.7 \%$ \\
\hline EWG-OL-2463-Q & $\mathrm{K}_{2} \mathrm{O}$ & 3.0928 & 3.0000 & 0.0928 & $3.1 \%$ \\
\hline EWG-OL-2463-Q & $\mathrm{Li}_{2} \mathrm{O}$ & 0.2153 & 0.0000 & 0.2153 & \\
\hline EWG-OL-2463-Q & $\mathrm{MgO}$ & 0.1658 & 0.0000 & 0.1658 & \\
\hline EWG-OL-2463-Q & $\mathrm{MnO}$ & 3.0666 & 3.0000 & 0.0666 & $2.2 \%$ \\
\hline EWG-OL-2463-Q & $\mathrm{Na}_{2} \mathrm{O}$ & 18.4676 & 18.0000 & 0.4676 & $2.6 \%$ \\
\hline EWG-OL-2463-Q & $\mathrm{NiO}$ & 0.3601 & 0.4000 & -0.0399 & $-10.0 \%$ \\
\hline EWG-OL-2463-Q & $\mathrm{P}_{2} \mathrm{O}_{5}$ & 2.7382 & 3.0000 & -0.2618 & $-8.7 \%$ \\
\hline EWG-OL-2463-Q & $\mathrm{PbO}$ & 0.2674 & 0.3000 & -0.0326 & $-10.9 \%$ \\
\hline EWG-OL-2463-Q & $\mathrm{RuO}_{2}$ & 0.0132 & 0.0100 & 0.0032 & $31.7 \%$ \\
\hline EWG-OL-2463-Q & $\mathrm{SiO}_{2}$ & 20.1362 & 20.0000 & 0.1362 & $0.7 \%$ \\
\hline EWG-OL-2463-Q & $\mathrm{SO}_{3}$ & 0.3258 & 0.3000 & 0.0258 & $8.6 \%$ \\
\hline EWG-OL-2463-Q & $\mathrm{SrO}$ & 0.1168 & 0.1200 & -0.0032 & $-2.7 \%$ \\
\hline EWG-OL-2463-Q & $\mathrm{ZrO}_{2}$ & 3.9004 & 4.0000 & -0.0996 & $-2.5 \%$ \\
\hline EWG-OL-2463-Q & Sum & 100.4843 & 99.6850 & 0.7993 & $0.8 \%$ \\
\hline EWG-OL-26012-Q & $\mathrm{Al}_{2} \mathrm{O}_{3}$ & 14.9507 & 15.0000 & -0.0493 & $-0.3 \%$ \\
\hline EWG-OL-26012-Q & $\mathrm{B}_{2} \mathrm{O}_{3}$ & 8.0095 & 8.0000 & 0.0095 & $0.1 \%$ \\
\hline EWG-OL-26012-Q & $\mathrm{Bi}_{2} \mathrm{O}_{3}$ & 3.0072 & 3.0000 & 0.0072 & $0.2 \%$ \\
\hline EWG-OL-26012-Q & $\mathrm{CaO}$ & 0.1399 & 0.0000 & 0.1399 & \\
\hline EWG-OL-26012-Q & $\mathrm{CdO}$ & 0.0868 & 0.1000 & -0.0132 & $-13.2 \%$ \\
\hline EWG-OL-26012-Q & $\mathrm{Cr}_{2} \mathrm{O}_{3}$ & 0.1462 & 0.0000 & 0.1462 & \\
\hline EWG-OL-26012-Q & $\mathrm{Fe}_{2} \mathrm{O}_{3}$ & 0.1430 & 0.0000 & 0.1430 & \\
\hline EWG-OL-26012-Q & $\mathrm{K}_{2} \mathrm{O}$ & 3.1289 & 3.0000 & 0.1289 & $4.3 \%$ \\
\hline EWG-OL-26012-Q & $\mathrm{Li}_{2} \mathrm{O}$ & 1.6744 & 1.6800 & -0.0056 & $-0.3 \%$ \\
\hline EWG-OL-26012-Q & $\mathrm{MgO}$ & 0.1658 & 0.0000 & 0.1658 & \\
\hline
\end{tabular}


Table A-4. Comparison of Measured and Targeted Compositions for Set 1 Study Glasses (continued)

\begin{tabular}{|c|c|c|c|c|c|}
\hline Glass ID & Oxide & $\begin{array}{l}\text { Measured } \\
\text { (wt \%) }\end{array}$ & $\begin{array}{l}\text { Targeted } \\
\text { (wt \%) }\end{array}$ & $\begin{array}{c}\text { Difference of Measured } \\
\text { versus Targeted }\end{array}$ & $\begin{array}{c}\text { \% Difference of Measured } \\
\text { versus Targeted }\end{array}$ \\
\hline EWG-OL-26012-Q & $\mathrm{MnO}$ & 0.1291 & 0.0000 & 0.1291 & \\
\hline EWG-OL-26012-Q & $\mathrm{Na}_{2} \mathrm{O}$ & 18.1306 & 18.0000 & 0.1306 & $0.7 \%$ \\
\hline EWG-OL-26012-Q & $\mathrm{NiO}$ & 0.3872 & 0.4000 & -0.0128 & $-3.2 \%$ \\
\hline EWG-OL-26012-Q & $\mathrm{P}_{2} \mathrm{O}_{5}$ & 2.8012 & 3.0000 & -0.1988 & $-6.6 \%$ \\
\hline EWG-OL-26012-Q & $\mathrm{PbO}$ & 0.2793 & 0.3000 & -0.0207 & $-6.9 \%$ \\
\hline EWG-OL-26012-Q & $\mathrm{RuO}_{2}$ & 0.0132 & 0.0100 & 0.0032 & $31.7 \%$ \\
\hline EWG-OL-26012-Q & $\mathrm{SiO}_{2}$ & 42.5721 & 42.7750 & -0.2029 & $-0.5 \%$ \\
\hline EWG-OL-26012-Q & $\mathrm{SO}_{3}$ & 0.2360 & 0.3000 & -0.0640 & $-21.3 \%$ \\
\hline EWG-OL-26012-Q & $\mathrm{SrO}$ & 0.1174 & 0.1200 & -0.0026 & $-2.2 \%$ \\
\hline EWG-OL-26012-Q & $\mathrm{ZrO}_{2}$ & 4.0017 & 4.0000 & 0.0017 & $0.0 \%$ \\
\hline EWG-OL-26012-Q & Sum & 100.1201 & 99.6850 & 0.4351 & $0.4 \%$ \\
\hline EWG-OL-2619-Q & $\mathrm{Al}_{2} \mathrm{O}_{3}$ & 18.5880 & 18.4550 & 0.1330 & $0.7 \%$ \\
\hline EWG-OL-2619-Q & $\mathrm{B}_{2} \mathrm{O}_{3}$ & 22.0724 & 22.0000 & 0.0724 & $0.3 \%$ \\
\hline EWG-OL-2619-Q & $\mathrm{Bi}_{2} \mathrm{O}_{3}$ & 3.1019 & 3.0000 & 0.1019 & $3.4 \%$ \\
\hline EWG-OL-2619-Q & $\mathrm{CaO}$ & 10.0917 & 10.0000 & 0.0917 & $0.9 \%$ \\
\hline EWG-OL-2619-Q & $\mathrm{CdO}$ & 0.0877 & 0.1000 & -0.0123 & $-12.3 \%$ \\
\hline EWG-OL-2619-Q & $\mathrm{Cr}_{2} \mathrm{O}_{3}$ & 0.1462 & 0.0000 & 0.1462 & \\
\hline EWG-OL-2619-Q & $\mathrm{Fe}_{2} \mathrm{O}_{3}$ & 10.1044 & 10.0000 & 0.1044 & $1.0 \%$ \\
\hline EWG-OL-2619-Q & $\mathrm{K}_{2} \mathrm{O}$ & 2.6351 & 3.0000 & -0.3649 & $-12.2 \%$ \\
\hline EWG-OL-2619-Q & $\mathrm{Li}_{2} \mathrm{O}$ & 0.2153 & 0.0000 & 0.2153 & \\
\hline EWG-OL-2619-Q & $\mathrm{MgO}$ & 0.1658 & 0.0000 & 0.1658 & \\
\hline EWG-OL-2619-Q & $\mathrm{MnO}$ & 3.1344 & 3.0000 & 0.1344 & $4.5 \%$ \\
\hline EWG-OL-2619-Q & $\mathrm{Na}_{2} \mathrm{O}$ & 4.9842 & 5.0000 & -0.0158 & $-0.3 \%$ \\
\hline EWG-OL-2619-Q & $\mathrm{NiO}$ & 0.2987 & 0.4000 & -0.1013 & $-25.3 \%$ \\
\hline EWG-OL-2619-Q & $\mathrm{P}_{2} \mathrm{O}_{5}$ & 0.2291 & 0.0000 & 0.2291 & \\
\hline EWG-OL-2619-Q & $\mathrm{PbO}$ & 0.2744 & 0.3000 & -0.0256 & $-8.5 \%$ \\
\hline EWG-OL-2619-Q & $\mathrm{RuO}_{2}$ & 0.0132 & 0.0100 & 0.0032 & $31.7 \%$ \\
\hline EWG-OL-2619-Q & $\mathrm{SiO}_{2}$ & 20.3608 & 20.0000 & 0.3608 & $1.8 \%$ \\
\hline EWG-OL-2619-Q & $\mathrm{SO}_{3}$ & 0.2160 & 0.3000 & -0.0840 & $-28.0 \%$ \\
\hline EWG-OL-2619-Q & $\mathrm{SrO}$ & 0.1186 & 0.1200 & -0.0014 & $-1.2 \%$ \\
\hline EWG-OL-2619-Q & $\mathrm{ZrO}_{2}$ & 2.5868 & 4.0000 & -1.4132 & $-35.3 \%$ \\
\hline EWG-OL-2619-Q & Sum & 99.4246 & 99.6850 & -0.2604 & $-0.3 \%$ \\
\hline EWG-OL-29285-Q & $\mathrm{Al}_{2} \mathrm{O}_{3}$ & 15.0546 & 15.0000 & 0.0546 & $0.4 \%$ \\
\hline EWG-OL-29285-Q & $\mathrm{B}_{2} \mathrm{O}_{3}$ & 18.4420 & 18.5762 & -0.1342 & $-0.7 \%$ \\
\hline EWG-OL-29285-Q & $\mathrm{Bi}_{2} \mathrm{O}_{3}$ & 0.1115 & 0.0000 & 0.1115 & \\
\hline EWG-OL-29285-Q & $\mathrm{CaO}$ & 10.2072 & 10.0000 & 0.2072 & $2.1 \%$ \\
\hline EWG-OL-29285-Q & $\mathrm{CdO}$ & 0.0862 & 0.1000 & -0.0138 & $-13.8 \%$ \\
\hline EWG-OL-29285-Q & $\mathrm{Cr}_{2} \mathrm{O}_{3}$ & 0.1462 & 0.0000 & 0.1462 & \\
\hline EWG-OL-29285-Q & $\mathrm{Fe}_{2} \mathrm{O}_{3}$ & 0.1430 & 0.0000 & 0.1430 & \\
\hline EWG-OL-29285-Q & $\mathrm{K}_{2} \mathrm{O}$ & 0.0120 & 0.0000 & 0.0120 & \\
\hline EWG-OL-29285-Q & $\mathrm{Li}_{2} \mathrm{O}$ & 0.2153 & 0.0000 & 0.2153 & \\
\hline EWG-OL-29285-Q & $\mathrm{MgO}$ & 3.4741 & 4.0000 & -0.5259 & $-13.1 \%$ \\
\hline EWG-OL-29285-Q & $\mathrm{MnO}$ & 0.1291 & 0.0000 & 0.1291 & \\
\hline EWG-OL-29285-Q & $\mathrm{Na}_{2} \mathrm{O}$ & 5.0617 & 5.0000 & 0.0617 & $1.2 \%$ \\
\hline EWG-OL-29285-Q & $\mathrm{NiO}$ & 0.3760 & 0.4000 & -0.0240 & $-6.0 \%$ \\
\hline EWG-OL-29285-Q & $\mathrm{P}_{2} \mathrm{O}_{5}$ & 2.8070 & 3.0000 & -0.1930 & $-6.4 \%$ \\
\hline EWG-OL-29285-Q & $\mathrm{PbO}$ & 0.2720 & 0.3000 & -0.0280 & $-9.3 \%$ \\
\hline EWG-OL-29285-Q & $\mathrm{RuO}_{2}$ & 0.0132 & 0.0100 & 0.0032 & $31.7 \%$ \\
\hline EWG-OL-29285-Q & $\mathrm{SiO}_{2}$ & 38.8818 & 38.8788 & 0.0030 & $0.0 \%$ \\
\hline EWG-OL-29285-Q & $\mathrm{SO}_{3}$ & 0.0449 & 0.3000 & -0.2551 & $-85.0 \%$ \\
\hline EWG-OL-29285-Q & $\mathrm{SrO}$ & 0.1200 & 0.1200 & 0.0000 & $0.0 \%$ \\
\hline EWG-OL-29285-Q & $\mathrm{ZrO}_{2}$ & 3.9950 & 4.0000 & -0.0050 & $-0.1 \%$ \\
\hline EWG-OL-29285-Q & Sum & 99.5928 & 99.6850 & -0.0922 & $-0.1 \%$ \\
\hline EWG-OL-31644-Q & $\mathrm{Al}_{2} \mathrm{O}_{3}$ & 15.2199 & 15.0000 & 0.2199 & $1.5 \%$ \\
\hline EWG-OL-31644-Q & $\mathrm{B}_{2} \mathrm{O}_{3}$ & 8.2027 & 8.0000 & 0.2027 & $2.5 \%$ \\
\hline EWG-OL-31644-Q & $\mathrm{Bi}_{2} \mathrm{O}_{3}$ & 0.1115 & 0.0000 & 0.1115 & \\
\hline EWG-OL-31644-Q & $\mathrm{CaO}$ & 10.5220 & 10.0000 & 0.5220 & $5.2 \%$ \\
\hline EWG-OL-31644-Q & $\mathrm{CdO}$ & 0.0840 & 0.1000 & -0.0160 & $-16.0 \%$ \\
\hline EWG-OL-31644-Q & $\mathrm{Cr}_{2} \mathrm{O}_{3}$ & 1.4532 & 1.6000 & -0.1468 & $-9.2 \%$ \\
\hline
\end{tabular}


Table A-4. Comparison of Measured and Targeted Compositions for Set 1 Study Glasses (continued)

\begin{tabular}{|c|c|c|c|c|c|}
\hline Glass ID & Oxide & $\begin{array}{l}\text { Measured } \\
\text { (wt \%) }\end{array}$ & $\begin{array}{c}\text { Targeted } \\
\text { (wt \%) }\end{array}$ & $\begin{array}{c}\text { Difference of Measured } \\
\text { versus Targeted }\end{array}$ & $\begin{array}{c}\text { \% Difference of Measured } \\
\text { versus Targeted }\end{array}$ \\
\hline EWG-OL-31644-Q & $\mathrm{Fe}_{2} \mathrm{O}_{3}$ & 9.9579 & 10.0000 & -0.0421 & $-0.4 \%$ \\
\hline EWG-OL-31644-Q & $\mathrm{K}_{2} \mathrm{O}$ & 0.0120 & 0.0000 & 0.0120 & \\
\hline EWG-OL-31644-Q & $\mathrm{Li}_{2} \mathrm{O}$ & 1.3962 & 1.3761 & 0.0201 & $1.5 \%$ \\
\hline EWG-OL-31644-Q & $\mathrm{MgO}$ & 3.7353 & 4.0000 & -0.2647 & $-6.6 \%$ \\
\hline EWG-OL-31644-Q & $\mathrm{MnO}$ & 3.0957 & 3.0000 & 0.0957 & $3.2 \%$ \\
\hline EWG-OL-31644-Q & $\mathrm{Na}_{2} \mathrm{O}$ & 4.9236 & 5.0000 & -0.0764 & $-1.5 \%$ \\
\hline EWG-OL-31644-Q & $\mathrm{NiO}$ & 0.2866 & 0.4000 & -0.1134 & $-28.3 \%$ \\
\hline EWG-OL-31644-Q & $\mathrm{P}_{2} \mathrm{O}_{5}$ & 0.2291 & 0.0000 & 0.2291 & \\
\hline EWG-OL-31644-Q & $\mathrm{PbO}$ & 0.2739 & 0.3000 & -0.0261 & $-8.7 \%$ \\
\hline EWG-OL-31644-Q & $\mathrm{RuO}_{2}$ & 0.0132 & 0.0100 & 0.0032 & $31.7 \%$ \\
\hline EWG-OL-31644-Q & $\mathrm{SiO}_{2}$ & 40.8606 & 40.4789 & 0.3817 & $0.9 \%$ \\
\hline EWG-OL-31644-Q & $\mathrm{SO}_{3}$ & 0.1248 & 0.3000 & -0.1752 & $-58.4 \%$ \\
\hline EWG-OL-31644-Q & $\mathrm{SrO}$ & 0.1203 & 0.1200 & 0.0003 & $0.3 \%$ \\
\hline EWG-OL-31644-Q & $\mathrm{ZrO}_{2}$ & 0.1351 & 0.0000 & 0.1351 & \\
\hline EWG-OL-31644-Q & Sum & 100.7575 & 99.6850 & 1.0725 & $1.1 \%$ \\
\hline EWG-OL-33115-Q & $\mathrm{Al}_{2} \mathrm{O}_{3}$ & 15.1680 & 15.0000 & 0.1680 & $1.1 \%$ \\
\hline EWG-OL-33115-Q & $\mathrm{B}_{2} \mathrm{O}_{3}$ & 8.2590 & 8.0000 & 0.2590 & $3.2 \%$ \\
\hline EWG-OL-33115-Q & $\mathrm{Bi}_{2} \mathrm{O}_{3}$ & 3.0936 & 3.0000 & 0.0936 & $3.1 \%$ \\
\hline EWG-OL-33115-Q & $\mathrm{CaO}$ & 9.7804 & 10.0000 & -0.2196 & $-2.2 \%$ \\
\hline EWG-OL-33115-Q & $\mathrm{CdO}$ & 0.0842 & 0.1000 & -0.0158 & $-15.8 \%$ \\
\hline EWG-OL-33115-Q & $\mathrm{Cr}_{2} \mathrm{O}_{3}$ & 0.1462 & 0.0000 & 0.1462 & \\
\hline EWG-OL-33115-Q & $\mathrm{Fe}_{2} \mathrm{O}_{3}$ & 10.0687 & 10.0000 & 0.0687 & $0.7 \%$ \\
\hline EWG-OL-33115-Q & $\mathrm{K}_{2} \mathrm{O}$ & 2.6260 & 3.0000 & -0.3740 & $-12.5 \%$ \\
\hline EWG-OL-33115-Q & $\mathrm{Li}_{2} \mathrm{O}$ & 0.2153 & 0.0000 & 0.2153 & \\
\hline EWG-OL-33115-Q & $\mathrm{MgO}$ & 3.6483 & 4.0000 & -0.3517 & $-8.8 \%$ \\
\hline EWG-OL-33115-Q & $\mathrm{MnO}$ & 0.1291 & 0.0000 & 0.1291 & \\
\hline EWG-OL-33115-Q & $\mathrm{Na}_{2} \mathrm{O}$ & 6.7265 & 6.5593 & 0.1672 & $2.5 \%$ \\
\hline EWG-OL-33115-Q & $\mathrm{NiO}$ & 0.3748 & 0.4000 & -0.0252 & $-6.3 \%$ \\
\hline EWG-OL-33115-Q & $\mathrm{P}_{2} \mathrm{O}_{5}$ & 2.7726 & 3.0000 & -0.2274 & $-7.6 \%$ \\
\hline EWG-OL-33115-Q & $\mathrm{PbO}$ & 0.2706 & 0.3000 & -0.0294 & $-9.8 \%$ \\
\hline EWG-OL-33115-Q & $\mathrm{RuO}_{2}$ & 0.0132 & 0.0100 & 0.0032 & $31.7 \%$ \\
\hline EWG-OL-33115-Q & $\mathrm{SiO}_{2}$ & 36.2077 & 35.8957 & 0.3120 & $0.9 \%$ \\
\hline EWG-OL-33115-Q & $\mathrm{SO}_{3}$ & 0.2029 & 0.3000 & -0.0971 & $-32.4 \%$ \\
\hline EWG-OL-33115-Q & $\mathrm{SrO}$ & 0.1112 & 0.1200 & -0.0088 & $-7.4 \%$ \\
\hline EWG-OL-33115-Q & $\mathrm{ZrO}_{2}$ & 0.1351 & 0.0000 & 0.1351 & \\
\hline EWG-OL-33115-Q & Sum & 100.0332 & 99.6850 & 0.3482 & $0.3 \%$ \\
\hline EWG-OL-38552-Q & $\mathrm{Al}_{2} \mathrm{O}_{3}$ & 25.9806 & 26.0000 & -0.0194 & $-0.1 \%$ \\
\hline EWG-OL-38552-Q & $\mathrm{B}_{2} \mathrm{O}_{3}$ & 8.2510 & 8.0000 & 0.2510 & $3.1 \%$ \\
\hline EWG-OL-38552-Q & $\mathrm{Bi}_{2} \mathrm{O}_{3}$ & 3.0824 & 3.0000 & 0.0824 & $2.7 \%$ \\
\hline EWG-OL-38552-Q & $\mathrm{CaO}$ & 0.1399 & 0.0000 & 0.1399 & \\
\hline EWG-OL-38552-Q & $\mathrm{CdO}$ & 0.0860 & 0.1000 & -0.0140 & $-14.0 \%$ \\
\hline EWG-OL-38552-Q & $\mathrm{Cr}_{2} \mathrm{O}_{3}$ & 0.1462 & 0.0000 & 0.1462 & \\
\hline EWG-OL-38552-Q & $\mathrm{Fe}_{2} \mathrm{O}_{3}$ & 3.6350 & 3.6173 & 0.0177 & $0.5 \%$ \\
\hline EWG-OL-38552-Q & $\mathrm{K}_{2} \mathrm{O}$ & 0.0120 & 0.0000 & 0.0120 & \\
\hline EWG-OL-38552-Q & $\mathrm{Li}_{2} \mathrm{O}$ & 5.9797 & 6.0000 & -0.0203 & $-0.3 \%$ \\
\hline EWG-OL-38552-Q & $\mathrm{MgO}$ & 3.6400 & 4.0000 & -0.3600 & $-9.0 \%$ \\
\hline EWG-OL-38552-Q & $\mathrm{MnO}$ & 3.0860 & 3.0000 & 0.0860 & $2.9 \%$ \\
\hline EWG-OL-38552-Q & $\mathrm{Na}_{2} \mathrm{O}$ & 5.0617 & 5.0000 & 0.0617 & $1.2 \%$ \\
\hline EWG-OL-38552-Q & $\mathrm{NiO}$ & 0.3709 & 0.4000 & -0.0291 & $-7.3 \%$ \\
\hline EWG-OL-38552-Q & $\mathrm{P}_{2} \mathrm{O}_{5}$ & 2.7440 & 3.0000 & -0.2560 & $-8.5 \%$ \\
\hline EWG-OL-38552-Q & $\mathrm{PbO}$ & 0.2717 & 0.3000 & -0.0283 & $-9.4 \%$ \\
\hline EWG-OL-38552-Q & $\mathrm{RuO}_{2}$ & 0.0132 & 0.0100 & 0.0032 & $31.7 \%$ \\
\hline EWG-OL-38552-Q & $\mathrm{SiO}_{2}$ & 33.1592 & 32.8377 & 0.3214 & $1.0 \%$ \\
\hline EWG-OL-38552-Q & $\mathrm{SO}_{3}$ & 0.2179 & 0.3000 & -0.0821 & $-27.4 \%$ \\
\hline EWG-OL-38552-Q & $\mathrm{SrO}$ & 0.1129 & 0.1200 & -0.0071 & $-5.9 \%$ \\
\hline EWG-OL-38552-Q & $\mathrm{ZrO}_{2}$ & 3.1676 & 4.0000 & -0.8324 & $-20.8 \%$ \\
\hline EWG-OL-38552-Q & Sum & 99.1578 & 99.6850 & -0.5272 & $-0.5 \%$ \\
\hline EWG-OL-3872-Q & $\mathrm{Al}_{2} \mathrm{O}_{3}$ & 14.7428 & 15.0000 & -0.2572 & $-1.7 \%$ \\
\hline EWG-OL-3872-Q & $\mathrm{B}_{2} \mathrm{O}_{3}$ & 8.0980 & 8.0000 & 0.0980 & $1.2 \%$ \\
\hline
\end{tabular}


Table A-4. Comparison of Measured and Targeted Compositions for Set 1 Study Glasses (continued)

\begin{tabular}{|c|c|c|c|c|c|}
\hline Glass ID & Oxide & $\begin{array}{c}\text { Measured } \\
\text { (wt \%) }\end{array}$ & $\begin{array}{c}\text { Targeted } \\
(\mathrm{wt} \%)\end{array}$ & $\begin{array}{c}\text { Difference of Measured } \\
\text { versus Targeted }\end{array}$ & $\begin{array}{c}\text { \% Difference of Measured } \\
\text { versus Targeted }\end{array}$ \\
\hline EWG-OL-3872-Q & $\mathrm{Bi}_{2} \mathrm{O}_{3}$ & 3.1298 & 3.0000 & 0.1298 & $4.3 \%$ \\
\hline EWG-OL-3872-Q & $\mathrm{CaO}$ & 9.8609 & 10.0000 & -0.1391 & $-1.4 \%$ \\
\hline EWG-OL-3872-Q & $\mathrm{CdO}$ & 0.0854 & 0.1000 & -0.0146 & $-14.6 \%$ \\
\hline EWG-OL-3872-Q & $\mathrm{Cr}_{2} \mathrm{O}_{3}$ & 0.1462 & 0.0000 & 0.1462 & \\
\hline EWG-OL-3872-Q & $\mathrm{Fe}_{2} \mathrm{O}_{3}$ & 0.1430 & 0.0000 & 0.1430 & \\
\hline EWG-OL-3872-Q & $\mathrm{K}_{2} \mathrm{O}$ & 0.0120 & 0.0000 & 0.0120 & \\
\hline EWG-OL-3872-Q & $\mathrm{Li}_{2} \mathrm{O}$ & 5.8667 & 6.0000 & -0.1333 & $-2.2 \%$ \\
\hline EWG-OL-3872-Q & $\mathrm{MgO}$ & 3.6897 & 4.0000 & -0.3103 & $-7.8 \%$ \\
\hline EWG-OL-3872-Q & $\mathrm{MnO}$ & 0.1291 & 0.0000 & 0.1291 & \\
\hline EWG-OL-3872-Q & $\mathrm{Na}_{2} \mathrm{O}$ & 18.5687 & 18.0000 & 0.5687 & $3.2 \%$ \\
\hline EWG-OL-3872-Q & $\mathrm{NiO}$ & 0.3703 & 0.4000 & -0.0297 & $-7.4 \%$ \\
\hline EWG-OL-3872-Q & $\mathrm{P}_{2} \mathrm{O}_{5}$ & 2.8127 & 3.0000 & -0.1873 & $-6.2 \%$ \\
\hline EWG-OL-3872-Q & $\mathrm{PbO}$ & 0.2575 & 0.3000 & -0.0425 & $-14.2 \%$ \\
\hline EWG-OL-3872-Q & $\mathrm{RuO}_{2}$ & 0.0132 & 0.0100 & 0.0032 & $31.7 \%$ \\
\hline EWG-OL-3872-Q & $\mathrm{SiO}_{2}$ & 31.0199 & 31.4550 & -0.4351 & $-1.4 \%$ \\
\hline EWG-OL-3872-Q & $\mathrm{SO}_{3}$ & 0.3059 & 0.3000 & 0.0059 & $2.0 \%$ \\
\hline EWG-OL-3872-Q & $\mathrm{SrO}$ & 0.1085 & 0.1200 & -0.0115 & $-9.6 \%$ \\
\hline EWG-OL-3872-Q & $\mathrm{ZrO}_{2}$ & 0.1351 & 0.0000 & 0.1351 & \\
\hline EWG-OL-3872-Q & Sum & 99.4952 & 99.6850 & -0.1898 & $-0.2 \%$ \\
\hline EWG-OL-5155-Q & $\mathrm{Al}_{2} \mathrm{O}_{3}$ & 19.7925 & 19.8550 & -0.0625 & $-0.3 \%$ \\
\hline EWG-OL-5155-Q & $\mathrm{B}_{2} \mathrm{O}_{3}$ & 22.1610 & 22.0000 & 0.1610 & $0.7 \%$ \\
\hline EWG-OL-5155-Q & $\mathrm{Bi}_{2} \mathrm{O}_{3}$ & 3.2998 & 3.0000 & 0.2998 & $10.0 \%$ \\
\hline EWG-OL-5155-Q & $\mathrm{CaO}$ & 0.1399 & 0.0000 & 0.1399 & \\
\hline EWG-OL-5155-Q & $\mathrm{CdO}$ & 0.0880 & 0.1000 & -0.0120 & $-12.0 \%$ \\
\hline EWG-OL-5155-Q & $\mathrm{Cr}_{2} \mathrm{O}_{3}$ & 1.0699 & 1.6000 & -0.5301 & $-33.1 \%$ \\
\hline EWG-OL-5155-Q & $\mathrm{Fe}_{2} \mathrm{O}_{3}$ & 9.2216 & 10.0000 & -0.7784 & $-7.8 \%$ \\
\hline EWG-OL-5155-Q & $\mathrm{K}_{2} \mathrm{O}$ & 0.0120 & 0.0000 & 0.0120 & \\
\hline EWG-OL-5155-Q & $\mathrm{Li}_{2} \mathrm{O}$ & 6.0281 & 6.0000 & 0.0281 & $0.5 \%$ \\
\hline EWG-OL-5155-Q & $\mathrm{MgO}$ & 3.5322 & 4.0000 & -0.4678 & $-11.7 \%$ \\
\hline EWG-OL-5155-Q & $\mathrm{MnO}$ & 2.9698 & 3.0000 & -0.0302 & $-1.0 \%$ \\
\hline EWG-OL-5155-Q & $\mathrm{Na}_{2} \mathrm{O}$ & 5.1056 & 5.0000 & 0.1056 & $2.1 \%$ \\
\hline EWG-OL-5155-Q & $\mathrm{NiO}$ & 0.1715 & 0.4000 & -0.2285 & $-57.1 \%$ \\
\hline EWG-OL-5155-Q & $\mathrm{P}_{2} \mathrm{O}_{5}$ & 0.2291 & 0.0000 & 0.2291 & \\
\hline EWG-OL-5155-Q & $\mathrm{PbO}$ & 0.2774 & 0.3000 & -0.0226 & $-7.5 \%$ \\
\hline EWG-OL-5155-Q & $\mathrm{RuO}_{2}$ & 0.0132 & 0.0100 & 0.0032 & $31.7 \%$ \\
\hline EWG-OL-5155-Q & $\mathrm{SiO}_{2}$ & 20.4089 & 20.0000 & 0.4089 & $2.0 \%$ \\
\hline EWG-OL-5155-Q & $\mathrm{SO}_{3}$ & 0.2391 & 0.3000 & -0.0609 & $-20.3 \%$ \\
\hline EWG-OL-5155-Q & $\mathrm{SrO}$ & 0.1177 & 0.1200 & -0.0023 & $-1.9 \%$ \\
\hline EWG-OL-5155-Q & $\mathrm{ZrO}_{2}$ & 3.9173 & 4.0000 & -0.0827 & $-2.1 \%$ \\
\hline EWG-OL-5155-Q & Sum & 98.7944 & 99.6850 & -0.8906 & $-0.9 \%$ \\
\hline EWG-OL-5801-Q & $\mathrm{Al}_{2} \mathrm{O}_{3}$ & 18.2337 & 18.4550 & -0.2213 & $-1.2 \%$ \\
\hline EWG-OL-5801-Q & $\mathrm{B}_{2} \mathrm{O}_{3}$ & 21.7021 & 22.0000 & -0.2979 & $-1.4 \%$ \\
\hline EWG-OL-5801-Q & $\mathrm{Bi}_{2} \mathrm{O}_{3}$ & 0.1115 & 0.0000 & 0.1115 & \\
\hline EWG-OL-5801-Q & $\mathrm{CaO}$ & 0.1399 & 0.0000 & 0.1399 & \\
\hline EWG-OL-5801-Q & $\mathrm{CdO}$ & 0.0874 & 0.1000 & -0.0126 & $-12.6 \%$ \\
\hline EWG-OL-5801-Q & $\mathrm{Cr}_{2} \mathrm{O}_{3}$ & 0.1462 & 0.0000 & 0.1462 & \\
\hline EWG-OL-5801-Q & $\mathrm{Fe}_{2} \mathrm{O}_{3}$ & 9.7148 & 10.0000 & -0.2852 & $-2.9 \%$ \\
\hline EWG-OL-5801-Q & $\mathrm{K}_{2} \mathrm{O}$ & 3.1320 & 3.0000 & 0.1320 & $4.4 \%$ \\
\hline EWG-OL-5801-Q & $\mathrm{Li}_{2} \mathrm{O}$ & 5.9420 & 6.0000 & -0.0580 & $-1.0 \%$ \\
\hline EWG-OL-5801-Q & $\mathrm{MgO}$ & 3.5819 & 4.0000 & -0.4181 & $-10.5 \%$ \\
\hline EWG-OL-5801-Q & $\mathrm{MnO}$ & 3.0408 & 3.0000 & 0.0408 & $1.4 \%$ \\
\hline EWG-OL-5801-Q & $\mathrm{Na}_{2} \mathrm{O}$ & 5.0921 & 5.0000 & 0.0921 & $1.8 \%$ \\
\hline EWG-OL-5801-Q & $\mathrm{NiO}$ & 0.3658 & 0.4000 & -0.0342 & $-8.5 \%$ \\
\hline EWG-OL-5801-Q & $\mathrm{P}_{2} \mathrm{O}_{5}$ & 2.7382 & 3.0000 & -0.2618 & $-8.7 \%$ \\
\hline EWG-OL-5801-Q & $\mathrm{PbO}$ & 0.2755 & 0.3000 & -0.0245 & $-8.2 \%$ \\
\hline EWG-OL-5801-Q & $\mathrm{RuO}_{2}$ & 0.0132 & 0.0100 & 0.0032 & $31.7 \%$ \\
\hline EWG-OL-5801-Q & $\mathrm{SiO}_{2}$ & 19.8634 & 20.0000 & -0.1366 & $-0.7 \%$ \\
\hline EWG-OL-5801-Q & $\mathrm{SO}_{3}$ & 0.3215 & 0.3000 & 0.0215 & $7.2 \%$ \\
\hline EWG-OL-5801-Q & $\mathrm{SrO}$ & 0.1162 & 0.1200 & -0.0038 & $-3.2 \%$ \\
\hline
\end{tabular}


Table A-4. Comparison of Measured and Targeted Compositions for Set 1 Study Glasses (continued)

\begin{tabular}{|c|c|c|c|c|c|}
\hline Glass ID & Oxide & $\begin{array}{c}\text { Measured } \\
\text { (wt \%) }\end{array}$ & $\begin{array}{c}\text { Targeted } \\
\text { (wt \%) }\end{array}$ & $\begin{array}{c}\text { Difference of Measured } \\
\text { versus Targeted }\end{array}$ & $\begin{array}{c}\text { \% Difference of Measured } \\
\text { versus Targeted }\end{array}$ \\
\hline EWG-OL-5801-Q & $\mathrm{ZrO}_{2}$ & 3.2757 & 4.0000 & -0.7243 & $-18.1 \%$ \\
\hline EWG-OL-5801-Q & Sum & 97.8938 & 99.6850 & -1.7912 & $-1.8 \%$ \\
\hline EWG-OL-6198-Q & $\mathrm{Al}_{2} \mathrm{O}_{3}$ & 15.0640 & 15.0000 & 0.0640 & $0.4 \%$ \\
\hline EWG-OL-6198-Q & $\mathrm{B}_{2} \mathrm{O}_{3}$ & 21.9195 & 22.0000 & -0.0805 & $-0.4 \%$ \\
\hline EWG-OL-6198-Q & $\mathrm{Bi}_{2} \mathrm{O}_{3}$ & 0.1115 & 0.0000 & 0.1115 & \\
\hline EWG-OL-6198-Q & $\mathrm{CaO}$ & 0.1399 & 0.0000 & 0.1399 & \\
\hline EWG-OL-6198-Q & $\mathrm{CdO}$ & 0.0865 & 0.1000 & -0.0135 & $-13.5 \%$ \\
\hline EWG-OL-6198-Q & $\mathrm{Cr}_{2} \mathrm{O}_{3}$ & 1.5274 & 1.6000 & -0.0726 & $-4.5 \%$ \\
\hline EWG-OL-6198-Q & $\mathrm{Fe}_{2} \mathrm{O}_{3}$ & 10.0186 & 10.0000 & 0.0186 & $0.2 \%$ \\
\hline EWG-OL-6198-Q & $\mathrm{K}_{2} \mathrm{O}$ & 3.0296 & 3.0000 & 0.0296 & $1.0 \%$ \\
\hline EWG-OL-6198-Q & $\mathrm{Li}_{2} \mathrm{O}$ & 5.9958 & 6.0000 & -0.0042 & $-0.1 \%$ \\
\hline EWG-OL-6198-Q & $\mathrm{MgO}$ & 3.5446 & 4.0000 & -0.4554 & $-11.4 \%$ \\
\hline EWG-OL-6198-Q & $\mathrm{MnO}$ & 0.1291 & 0.0000 & 0.1291 & \\
\hline EWG-OL-6198-Q & $\mathrm{Na}_{2} \mathrm{O}$ & 4.9573 & 5.0000 & -0.0427 & $-0.9 \%$ \\
\hline EWG-OL-6198-Q & $\mathrm{NiO}$ & 0.0964 & 0.4000 & -0.3036 & $-75.9 \%$ \\
\hline EWG-OL-6198-Q & $\mathrm{P}_{2} \mathrm{O}_{5}$ & 2.7611 & 3.0000 & -0.2389 & $-8.0 \%$ \\
\hline EWG-OL-6198-Q & $\mathrm{PbO}$ & 0.2768 & 0.3000 & -0.0232 & $-7.7 \%$ \\
\hline EWG-OL-6198-Q & $\mathrm{RuO}_{2}$ & 0.0132 & 0.0100 & 0.0032 & $31.7 \%$ \\
\hline EWG-OL-6198-Q & $\mathrm{SiO}_{2}$ & 25.0298 & 24.8550 & 0.1748 & $0.7 \%$ \\
\hline EWG-OL-6198-Q & $\mathrm{SO}_{3}$ & 0.3140 & 0.3000 & 0.0140 & $4.7 \%$ \\
\hline EWG-OL-6198-Q & $\mathrm{SrO}$ & 0.1165 & 0.1200 & -0.0035 & $-2.9 \%$ \\
\hline EWG-OL-6198-Q & $\mathrm{ZrO}_{2}$ & 3.7046 & 4.0000 & -0.2954 & $-7.4 \%$ \\
\hline EWG-OL-6198-Q & Sum & 98.8362 & 99.6850 & -0.8488 & $-0.9 \%$ \\
\hline EWG-OL-801-Q & $\mathrm{Al}_{2} \mathrm{O}_{3}$ & 29.5234 & 30.0000 & -0.4766 & $-1.6 \%$ \\
\hline EWG-OL-801-Q & $\mathrm{B}_{2} \mathrm{O}_{3}$ & 20.9937 & 21.4550 & -0.4613 & $-2.1 \%$ \\
\hline EWG-OL-801-Q & $\mathrm{Bi}_{2} \mathrm{O}_{3}$ & 2.9654 & 3.0000 & -0.0346 & $-1.2 \%$ \\
\hline EWG-OL-801-Q & $\mathrm{CaO}$ & 0.1399 & 0.0000 & 0.1399 & \\
\hline EWG-OL-801-Q & $\mathrm{CdO}$ & 0.0868 & 0.1000 & -0.0132 & $-13.2 \%$ \\
\hline EWG-OL-801-Q & $\mathrm{Cr}_{2} \mathrm{O}_{3}$ & 0.1462 & 0.0000 & 0.1462 & \\
\hline EWG-OL-801-Q & $\mathrm{Fe}_{2} \mathrm{O}_{3}$ & 0.1430 & 0.0000 & 0.1430 & \\
\hline EWG-OL-801-Q & $\mathrm{K}_{2} \mathrm{O}$ & 0.0120 & 0.0000 & 0.0120 & \\
\hline EWG-OL-801-Q & $\mathrm{Li}_{2} \mathrm{O}$ & 0.2153 & 0.0000 & 0.2153 & \\
\hline EWG-OL-801-Q & $\mathrm{MgO}$ & 0.1658 & 0.0000 & 0.1658 & \\
\hline EWG-OL-801-Q & $\mathrm{MnO}$ & 3.0150 & 3.0000 & 0.0150 & $0.5 \%$ \\
\hline EWG-OL-801-Q & $\mathrm{Na}_{2} \mathrm{O}$ & 17.8610 & 18.0000 & -0.1390 & $-0.8 \%$ \\
\hline EWG-OL-801-Q & $\mathrm{NiO}$ & 0.3770 & 0.4000 & -0.0230 & $-5.8 \%$ \\
\hline EWG-OL-801-Q & $\mathrm{P}_{2} \mathrm{O}_{5}$ & 2.7497 & 3.0000 & -0.2503 & $-8.3 \%$ \\
\hline EWG-OL-801-Q & $\mathrm{PbO}$ & 0.2750 & 0.3000 & -0.0250 & $-8.3 \%$ \\
\hline EWG-OL-801-Q & $\mathrm{RuO}_{2}$ & 0.0132 & 0.0100 & 0.0032 & $31.7 \%$ \\
\hline EWG-OL-801-Q & $\mathrm{SiO}_{2}$ & 19.7404 & 20.0000 & -0.2596 & $-1.3 \%$ \\
\hline EWG-OL-801-Q & $\mathrm{SO}_{3}$ & 0.2129 & 0.3000 & -0.0871 & $-29.0 \%$ \\
\hline EWG-OL-801-Q & $\mathrm{SrO}$ & 0.1150 & 0.1200 & -0.0050 & $-4.2 \%$ \\
\hline EWG-OL-801-Q & $\mathrm{ZrO}_{2}$ & 0.1351 & 0.0000 & 0.1351 & \\
\hline EWG-OL-801-Q & Sum & 98.8857 & 99.6850 & -0.7993 & $-0.8 \%$ \\
\hline LRM & $\mathrm{Al}_{2} \mathrm{O}_{3}$ & 9.7671 & 10.0000 & -0.2329 & $-2.3 \%$ \\
\hline LRM & $\mathrm{B}_{2} \mathrm{O}_{3}$ & 7.8861 & 8.0000 & -0.1139 & $-1.4 \%$ \\
\hline LRM & $\mathrm{Bi}_{2} \mathrm{O}_{3}$ & 0.1115 & 0.0000 & 0.1115 & \\
\hline LRM & $\mathrm{CaO}$ & 0.3111 & 0.5000 & -0.1889 & $-37.8 \%$ \\
\hline LRM & $\mathrm{CdO}$ & 0.1235 & 0.2000 & -0.0765 & $-38.3 \%$ \\
\hline LRM & $\mathrm{Cr}_{2} \mathrm{O}_{3}$ & 0.2990 & 0.2000 & 0.0990 & $49.5 \%$ \\
\hline LRM & $\mathrm{Fe}_{2} \mathrm{O}_{3}$ & 1.4221 & 1.0000 & 0.4221 & $42.2 \%$ \\
\hline LRM & $\mathrm{K}_{2} \mathrm{O}$ & 1.4706 & 1.5000 & -0.0294 & $-2.0 \%$ \\
\hline LRM & $\mathrm{Li}_{2} \mathrm{O}$ & 0.2153 & 0.1000 & 0.1153 & $115.3 \%$ \\
\hline LRM & $\mathrm{MgO}$ & 0.1658 & 0.1000 & 0.0658 & $65.8 \%$ \\
\hline LRM & $\mathrm{MnO}$ & 0.1291 & 0.1000 & 0.0291 & $29.1 \%$ \\
\hline LRM & $\mathrm{Na}_{2} \mathrm{O}$ & 21.5455 & 20.0000 & 1.5455 & $7.7 \%$ \\
\hline LRM & $\mathrm{NiO}$ & 0.1564 & 0.1000 & 0.0564 & $56.4 \%$ \\
\hline LRM & $\mathrm{P}_{2} \mathrm{O}_{5}$ & 0.4909 & 0.5000 & -0.0091 & $-1.8 \%$ \\
\hline LRM & $\mathrm{PbO}$ & 0.0707 & 0.1000 & -0.0293 & $-29.3 \%$ \\
\hline
\end{tabular}


Table A-4. Comparison of Measured and Targeted Compositions for Set 1 Study Glasses (continued)

\begin{tabular}{|c|c|c|c|c|c|}
\hline Glass ID & Oxide & $\begin{array}{l}\text { Measured } \\
\text { (wt \%) }\end{array}$ & $\begin{array}{l}\text { Targeted } \\
\text { (wt \%) }\end{array}$ & $\begin{array}{c}\text { Difference of Measured } \\
\text { versus Targeted }\end{array}$ & $\begin{array}{c}\text { \% Difference of Measured } \\
\text { versus Targeted }\end{array}$ \\
\hline LRM & $\mathrm{RuO}_{2}$ & 0.0132 & 0.0000 & 0.0132 & \\
\hline LRM & $\mathrm{SiO}_{2}$ & 53.9104 & 54.0000 & -0.0896 & $-0.2 \%$ \\
\hline LRM & $\mathrm{SO}_{3}$ & 0.2507 & 0.2000 & 0.0507 & $25.4 \%$ \\
\hline LRM & $\mathrm{SrO}$ & 0.0118 & 0.0000 & 0.0118 & \\
\hline LRM & $\mathrm{ZrO}_{2}$ & 0.9262 & 1.0000 & -0.0738 & $-7.4 \%$ \\
\hline LRM & Sum & 99.2771 & 97.6000 & 1.6771 & $1.7 \%$ \\
\hline RSM-EWG-1-034-Q & $\mathrm{Al}_{2} \mathrm{O}_{3}$ & 23.9022 & 23.9700 & -0.0678 & $-0.3 \%$ \\
\hline RSM-EWG-1-034-Q & $\mathrm{B}_{2} \mathrm{O}_{3}$ & 18.4017 & 19.1900 & -0.7883 & $-4.1 \%$ \\
\hline RSM-EWG-1-034-Q & $\mathrm{Bi}_{2} \mathrm{O}_{3}$ & 1.0900 & 1.1400 & -0.0500 & $-4.4 \%$ \\
\hline RSM-EWG-1-034-Q & $\mathrm{CaO}$ & 5.6773 & 5.5800 & 0.0973 & $1.7 \%$ \\
\hline RSM-EWG-1-034-Q & $\mathrm{CdO}$ & 0.0166 & 0.0200 & -0.0034 & $-17.2 \%$ \\
\hline RSM-EWG-1-034-Q & $\mathrm{Cr}_{2} \mathrm{O}_{3}$ & 0.3172 & 0.5200 & -0.2028 & $-39.0 \%$ \\
\hline RSM-EWG-1-034-Q & $\mathrm{Fe}_{2} \mathrm{O}_{3}$ & 5.9904 & 5.9000 & 0.0904 & $1.5 \%$ \\
\hline RSM-EWG-1-034-Q & $\mathrm{K}_{2} \mathrm{O}$ & 0.0952 & 0.1400 & -0.0448 & $-32.0 \%$ \\
\hline RSM-EWG-1-034-Q & $\mathrm{Li}_{2} \mathrm{O}$ & 3.6061 & 3.5700 & 0.0361 & $1.0 \%$ \\
\hline RSM-EWG-1-034-Q & $\mathrm{MgO}$ & 0.1658 & 0.1200 & 0.0458 & $38.2 \%$ \\
\hline RSM-EWG-1-034-Q & $\mathrm{MnO}$ & 0.1291 & 0.0000 & 0.1291 & \\
\hline RSM-EWG-1-034-Q & $\mathrm{Na}_{2} \mathrm{O}$ & 9.6449 & 9.5800 & 0.0649 & $0.7 \%$ \\
\hline RSM-EWG-1-034-Q & $\mathrm{NiO}$ & 0.1482 & 0.4000 & -0.2518 & $-62.9 \%$ \\
\hline RSM-EWG-1-034-Q & $\mathrm{P}_{2} \mathrm{O}_{5}$ & 1.0008 & 1.0500 & -0.0492 & $-4.7 \%$ \\
\hline RSM-EWG-1-034-Q & $\mathrm{PbO}$ & 0.3630 & 0.4100 & -0.0470 & $-11.5 \%$ \\
\hline RSM-EWG-1-034-Q & $\mathrm{RuO}_{2}$ & 0.0132 & 0.0000 & 0.0132 & \\
\hline RSM-EWG-1-034-Q & $\mathrm{SiO}_{2}$ & 28.0783 & 27.0000 & 1.0783 & $4.0 \%$ \\
\hline RSM-EWG-1-034-Q & $\mathrm{SO}_{3}$ & 0.1298 & 0.2000 & -0.0702 & $-35.1 \%$ \\
\hline RSM-EWG-1-034-Q & $\mathrm{SrO}$ & 0.0118 & 0.0000 & 0.0118 & \\
\hline RSM-EWG-1-034-Q & $\mathrm{ZrO}_{2}$ & 0.3725 & 0.3900 & -0.0175 & $-4.5 \%$ \\
\hline RSM-EWG-1-034-Q & Sum & 99.1541 & 99.1800 & -0.0259 & $0.0 \%$ \\
\hline
\end{tabular}




\section{Exhibit A-1. Plots of Oxide Measurements in Analytical Sequence Grouped by Analytical Block}

Oxide=Al2O3 (wt\%), Prep Method=PF

Variability Chart for measured

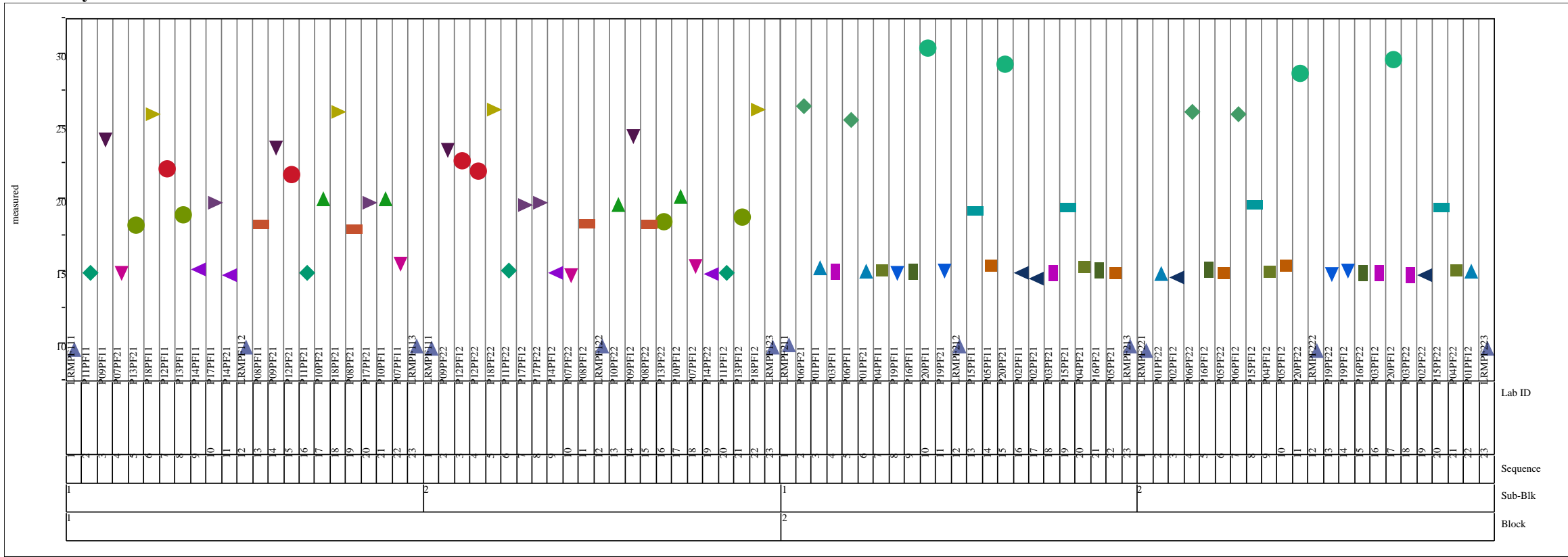




\section{Exhibit A-1. Plots of Oxide Measurements in Analytical Sequence Grouped by Analytical Block (continued)}

Oxide=B2O3 (wt \%), Prep Method=PF

Variability Chart for measured

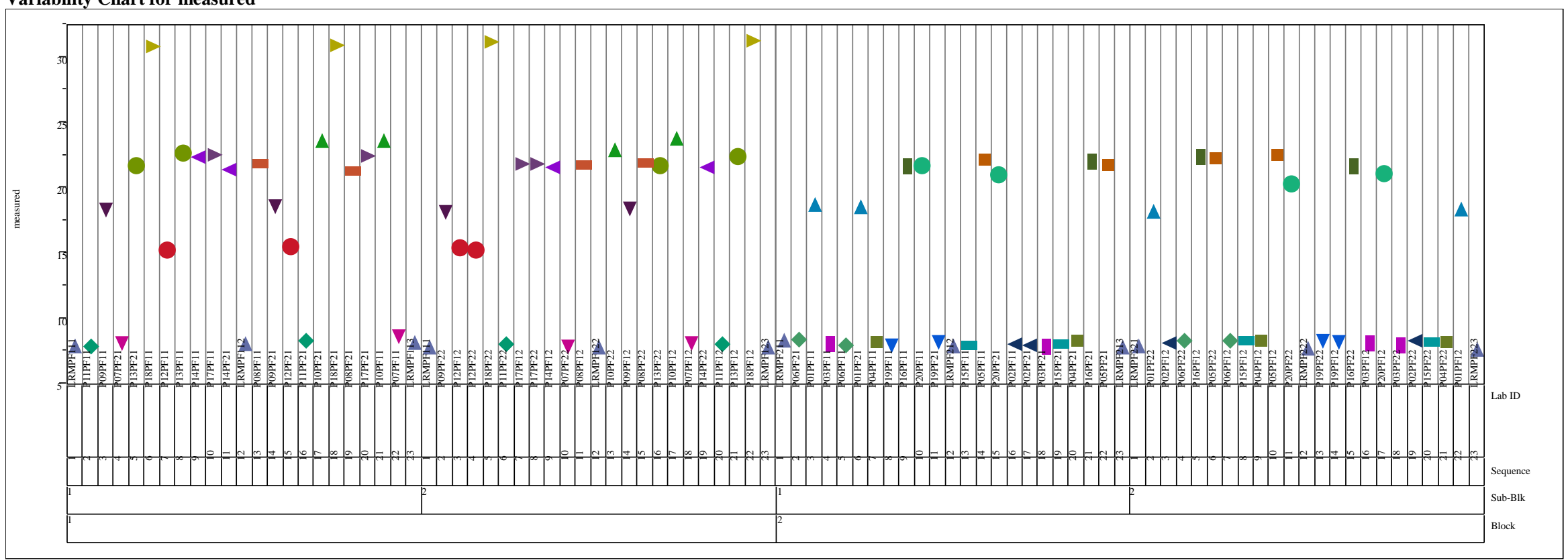




\section{Exhibit A-1. Plots of Oxide Measurements in Analytical Sequence Grouped by Analytical Block (continued)}

Oxide=Bi2O3 (wt\%), Prep Method=AD

Variability Chart for measured

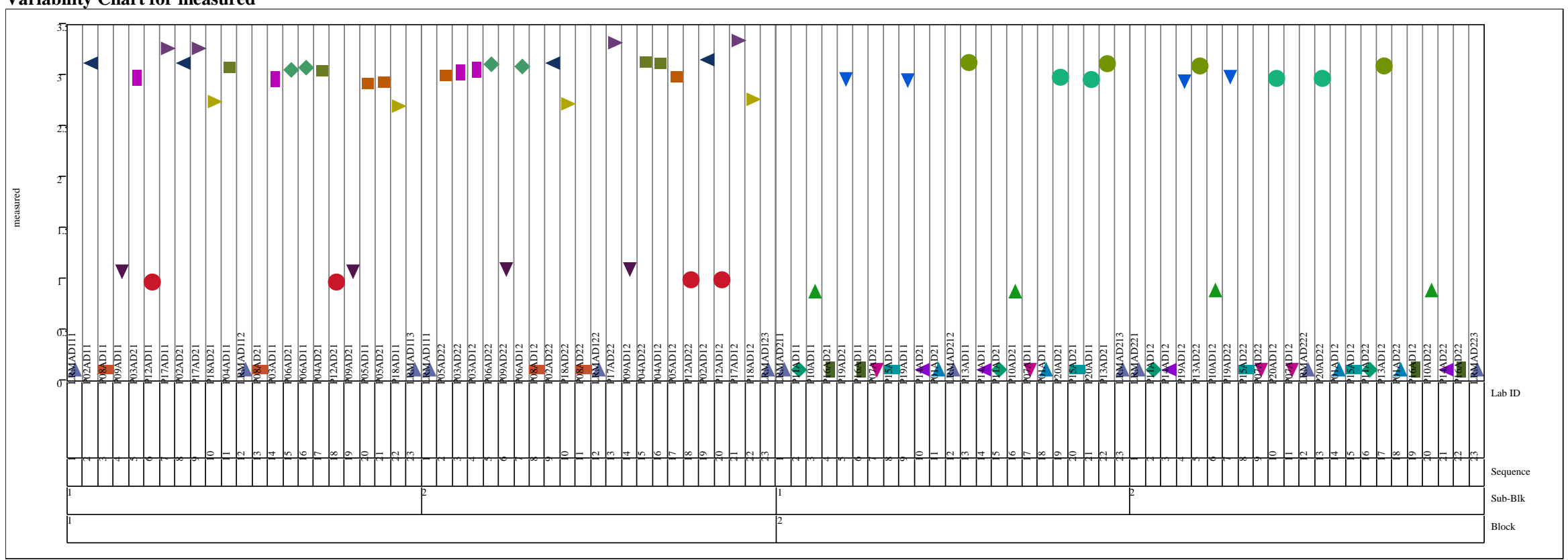


Exhibit A-1. Plots of Oxide Measurements in Analytical Sequence Grouped by Analytical Block (continued)

Oxide=CaO (wt \%), Prep Method $=$ PF

Variability Chart for measured

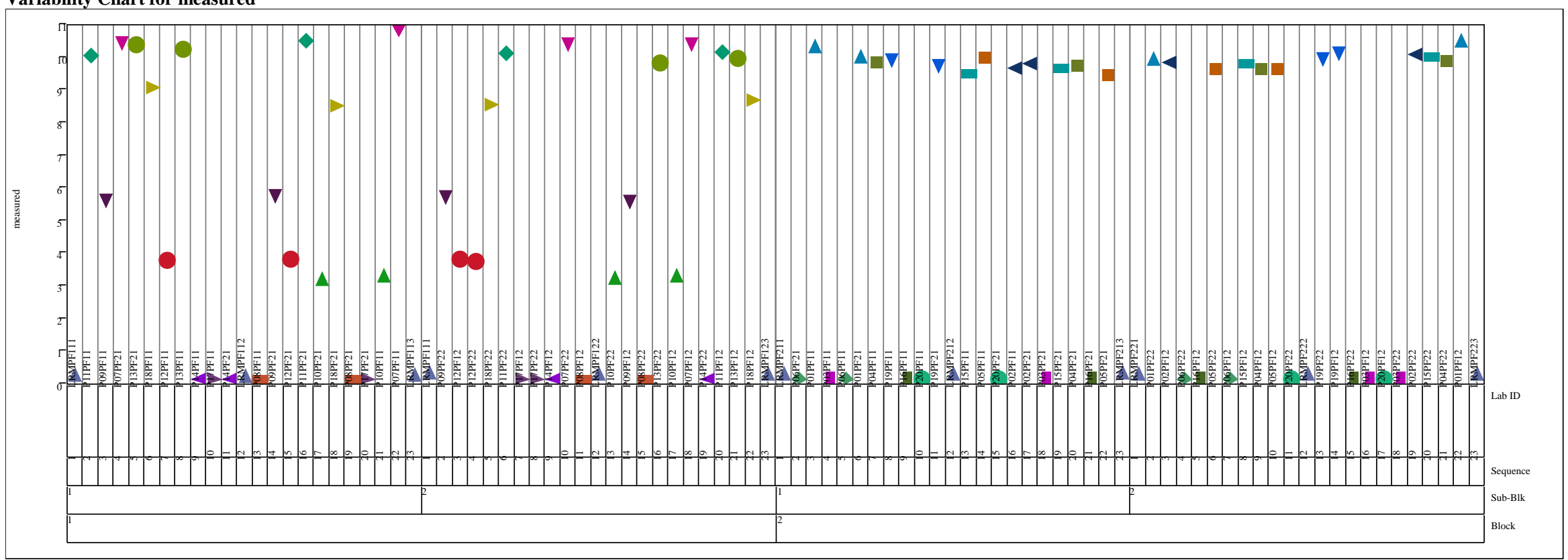




\section{Exhibit A-1. Plots of Oxide Measurements in Analytical Sequence Grouped by Analytical Block (continued)}

Oxide $=$ CdO (wt $\%$ ), Prep Method $=A D$

Variability Chart for measured

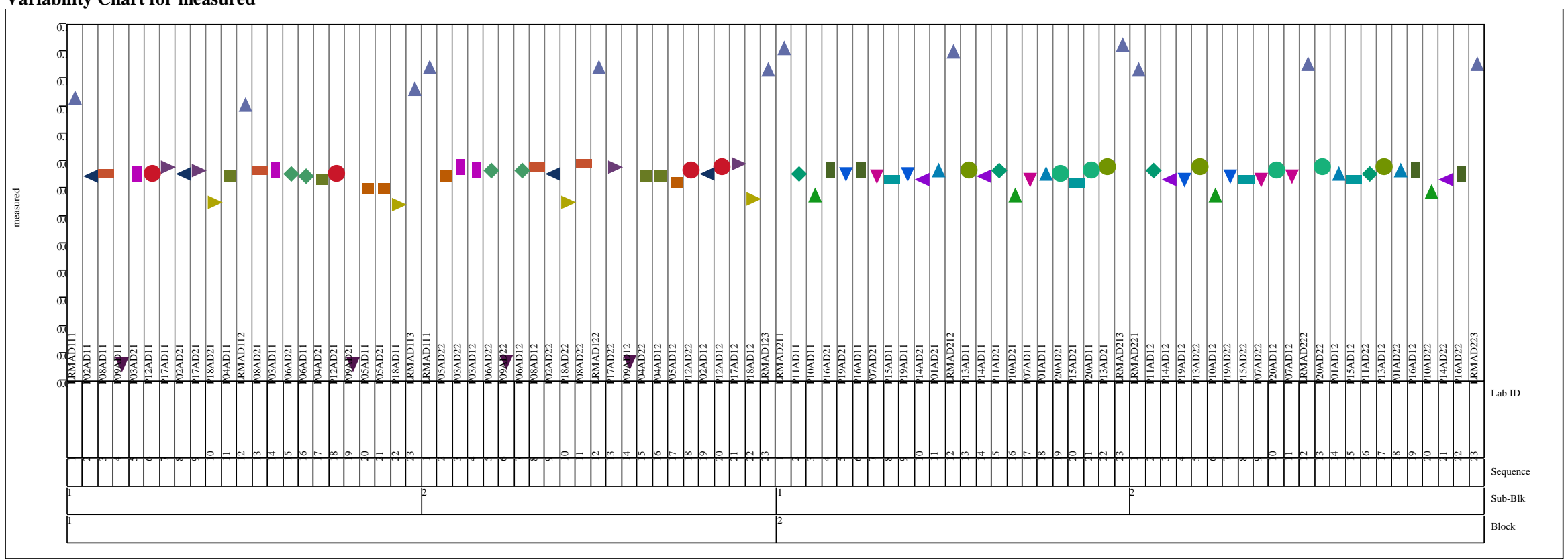




\section{Exhibit A-1. Plots of Oxide Measurements in Analytical Sequence Grouped by Analytical Block (continued)}

Oxide $=\mathrm{Cr} 2 \mathrm{O} 3$ (wt\%), Prep Method $=$ PF

Variability Chart for measured

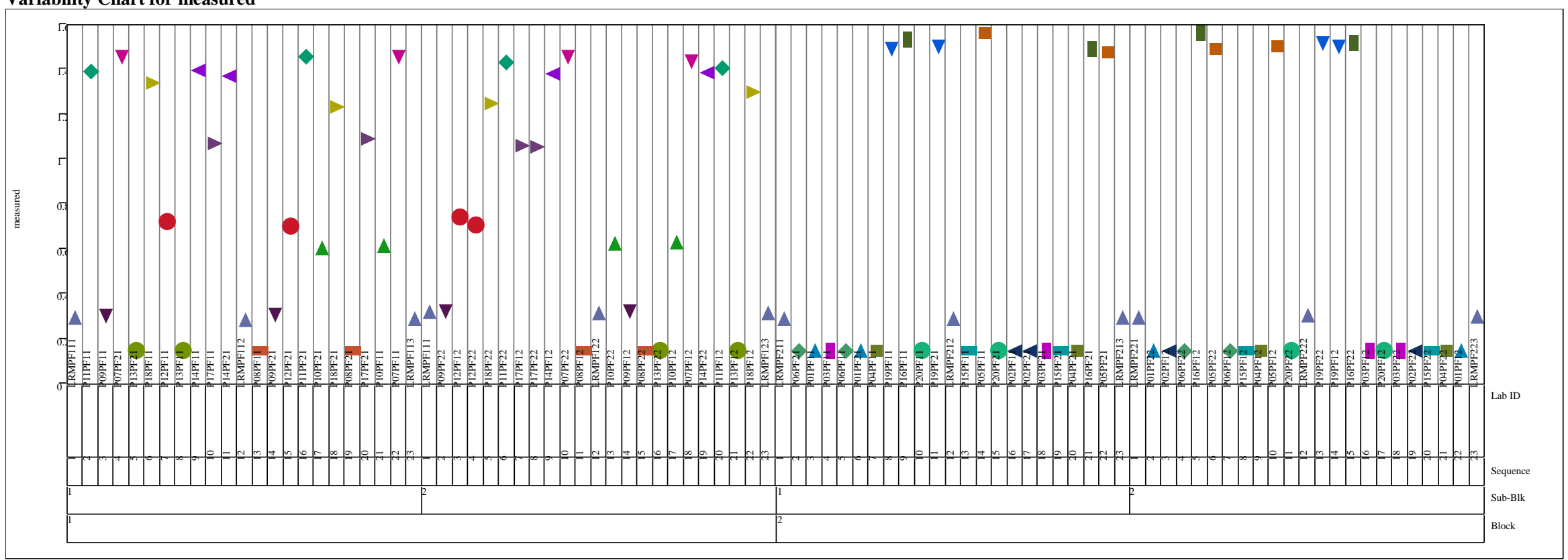




\section{Exhibit A-1. Plots of Oxide Measurements in Analytical Sequence Grouped by Analytical Block (continued)}

Oxide=Fe2O3 (wt \%), Prep Method=PF

Variability Chart for measured

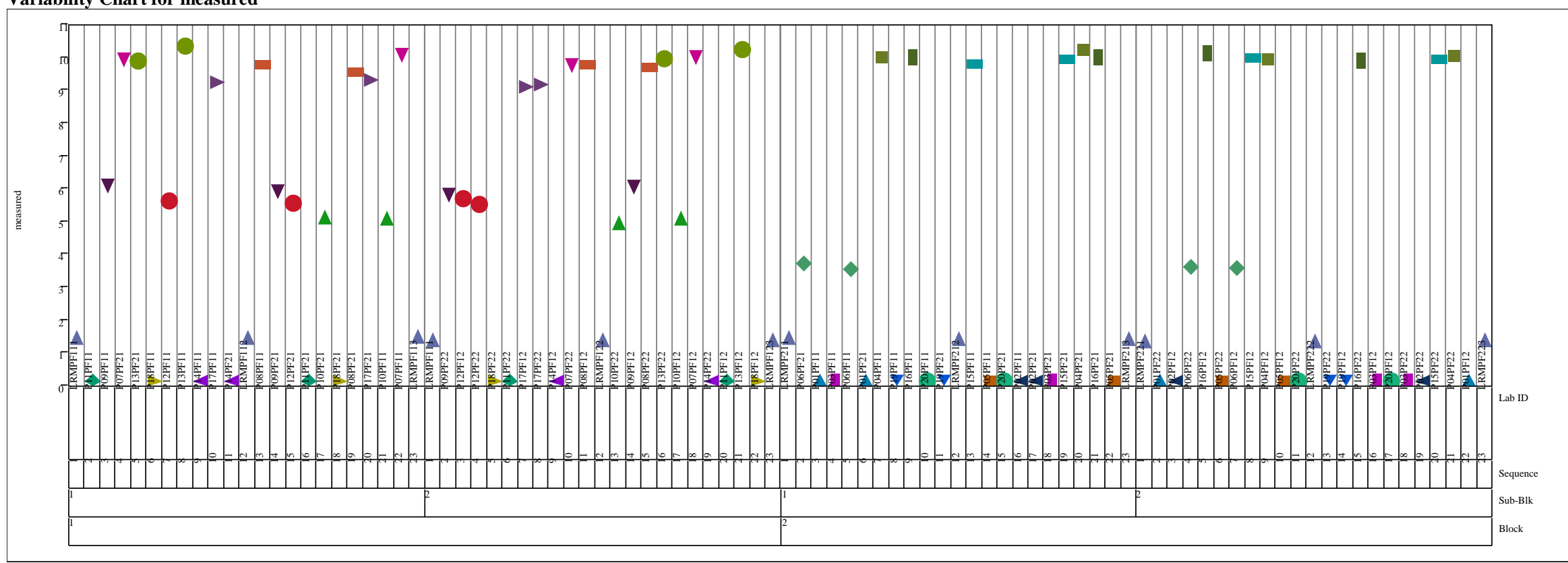




\section{Exhibit A-1. Plots of Oxide Measurements in Analytical Sequence Grouped by Analytical Block (continued)}

Oxide=K2O (wt \%), Prep Method=AD

Variability Chart for measured

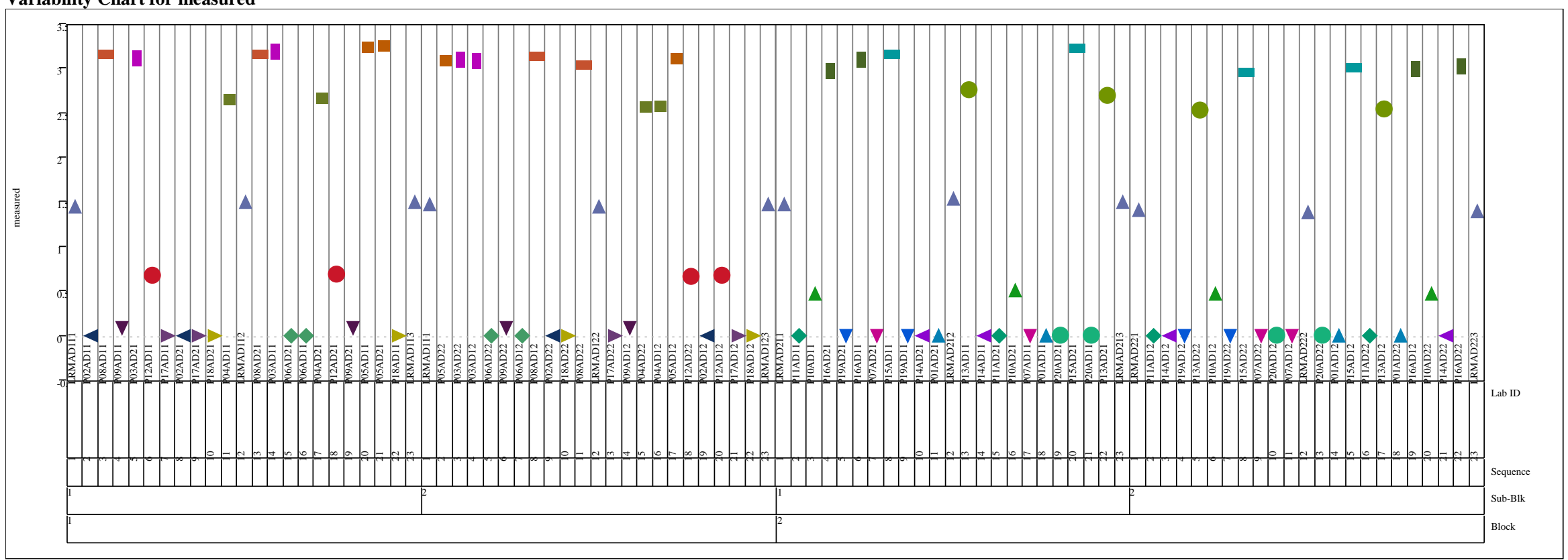




\section{Exhibit A-1. Plots of Oxide Measurements in Analytical Sequence Grouped by Analytical Block (continued)}

Oxide=Li2O (wt\%), Prep Method=PF

Variability Chart for measured

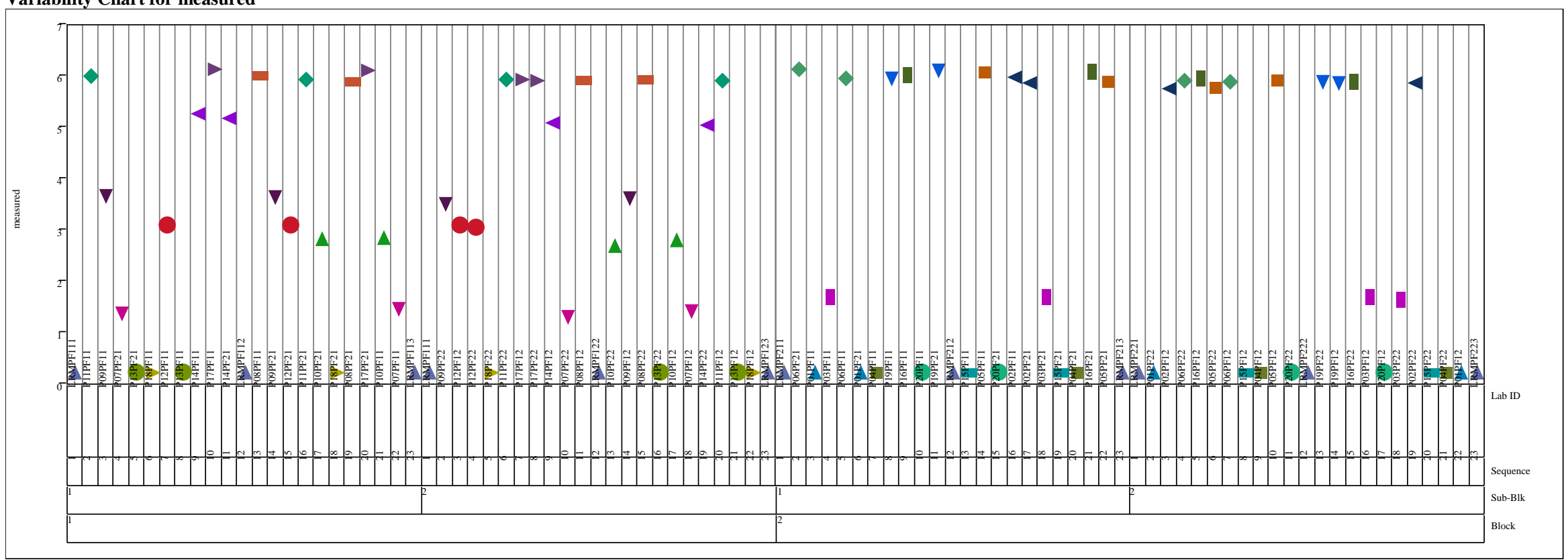




\section{Exhibit A-1. Plots of Oxide Measurements in Analytical Sequence Grouped by Analytical Block (continued)}

Oxide=MgO (wt\%), Prep Method $=$ PF

Variability Chart for measured

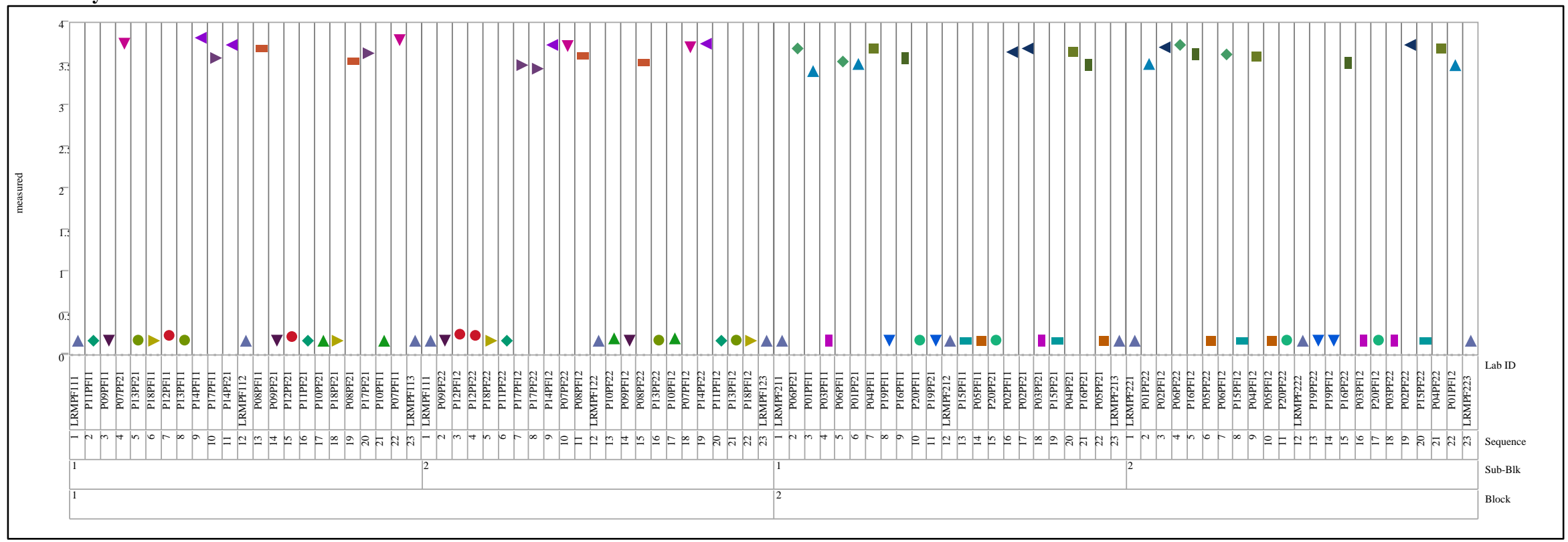




\section{Exhibit A-1. Plots of Oxide Measurements in Analytical Sequence Grouped by Analytical Block (continued)}

Oxide=MnO (wt\%), Prep Method=PF

Variability Chart for measured

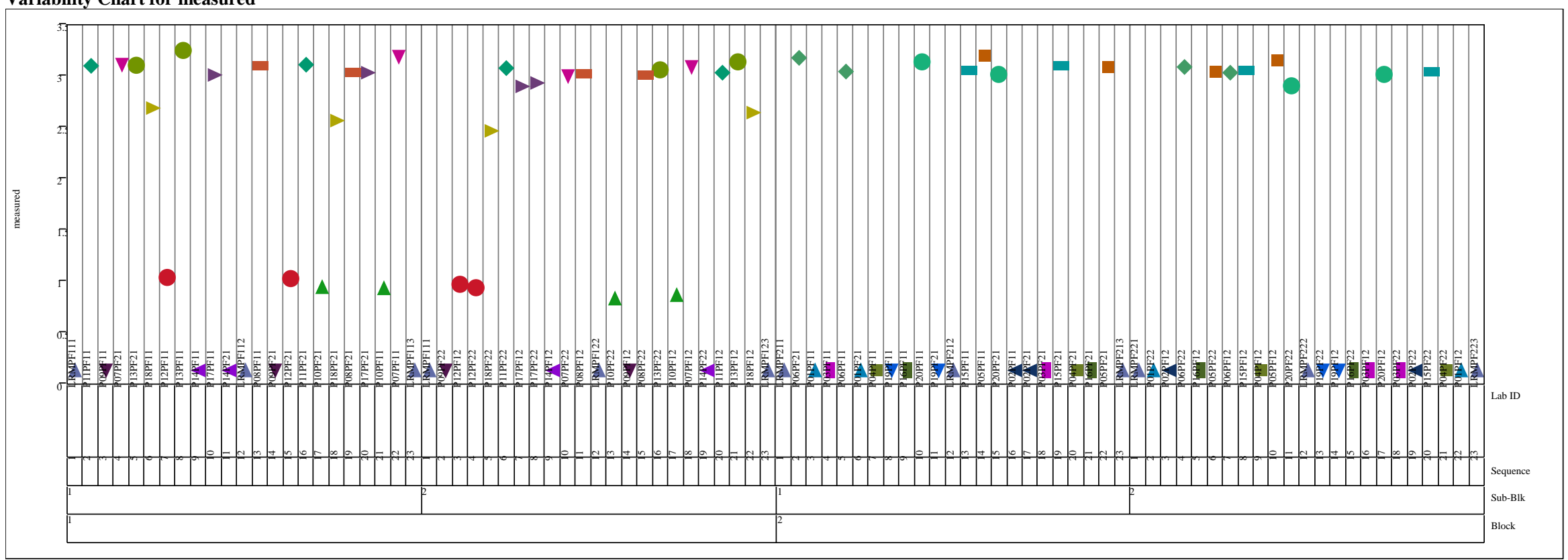




\section{Exhibit A-1. Plots of Oxide Measurements in Analytical Sequence Grouped by Analytical Block (continued)}

Oxide $=$ Na2O (wt $\%$ ), Prep Method $=A D$

Variability Chart for measured

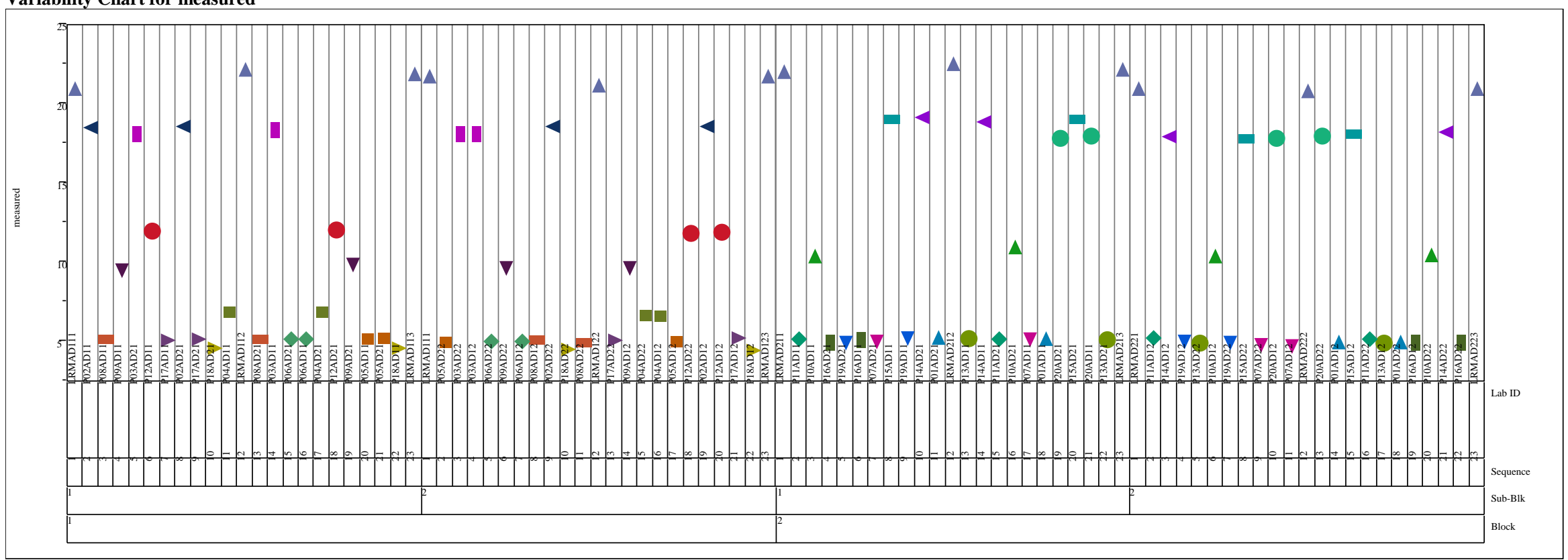




\section{Exhibit A-1. Plots of Oxide Measurements in Analytical Sequence Grouped by Analytical Block (continued)}

Oxide=NiO (wt \%), Prep Method=AD

Variability Chart for measured

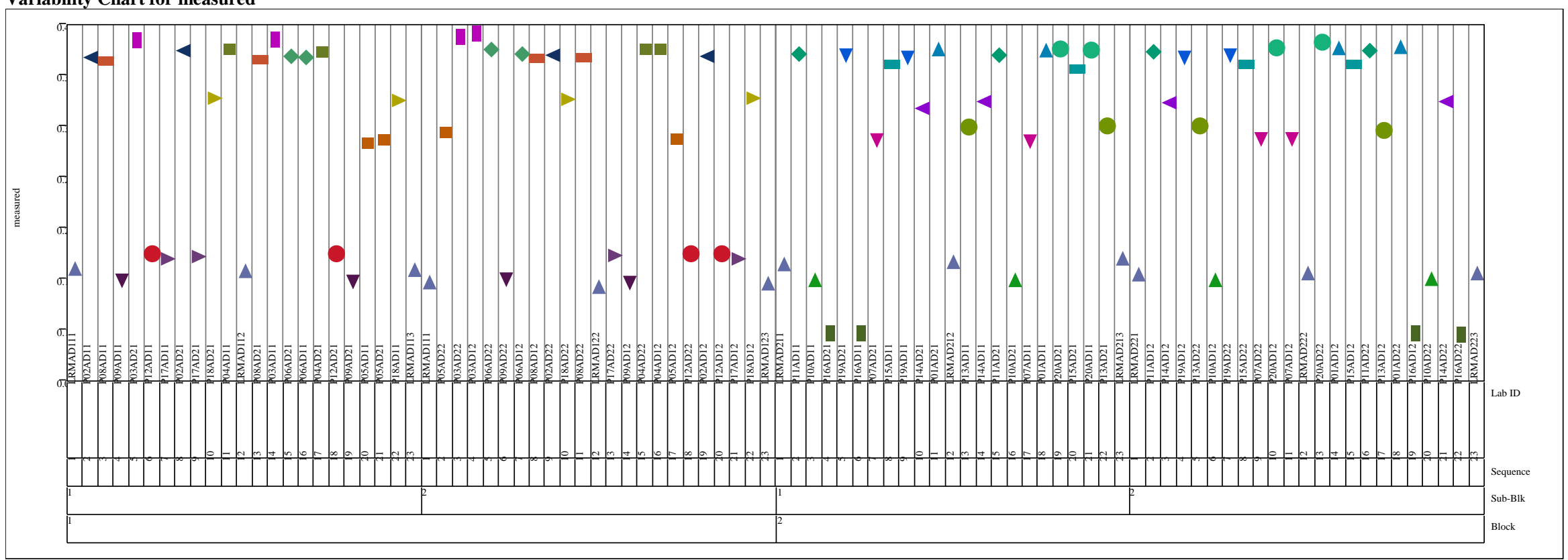


Exhibit A-1. Plots of Oxide Measurements in Analytical Sequence Grouped by Analytical Block (continued)

Oxide=P2O5 (wt\%), Prep Method=AD

Variability Chart for measured

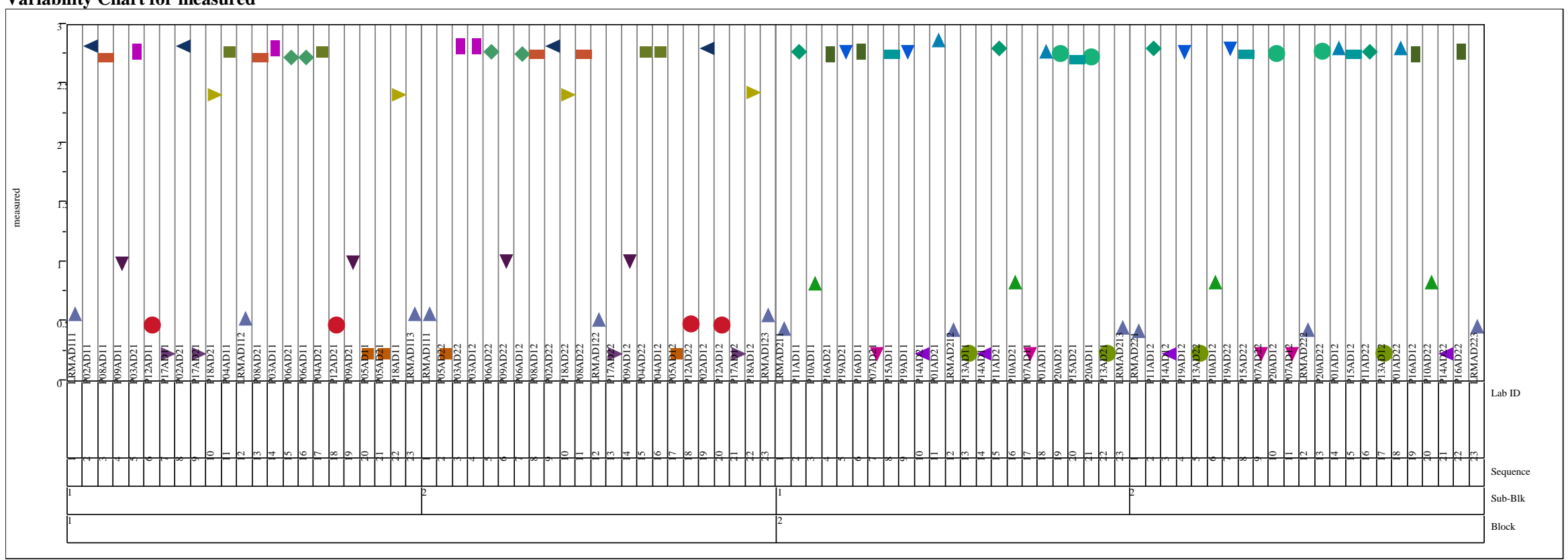




\section{Exhibit A-1. Plots of Oxide Measurements in Analytical Sequence Grouped by Analytical Block (continued)}

Oxide=PbO (wt \%), Prep Method $=$ AD

Variability Chart for measured

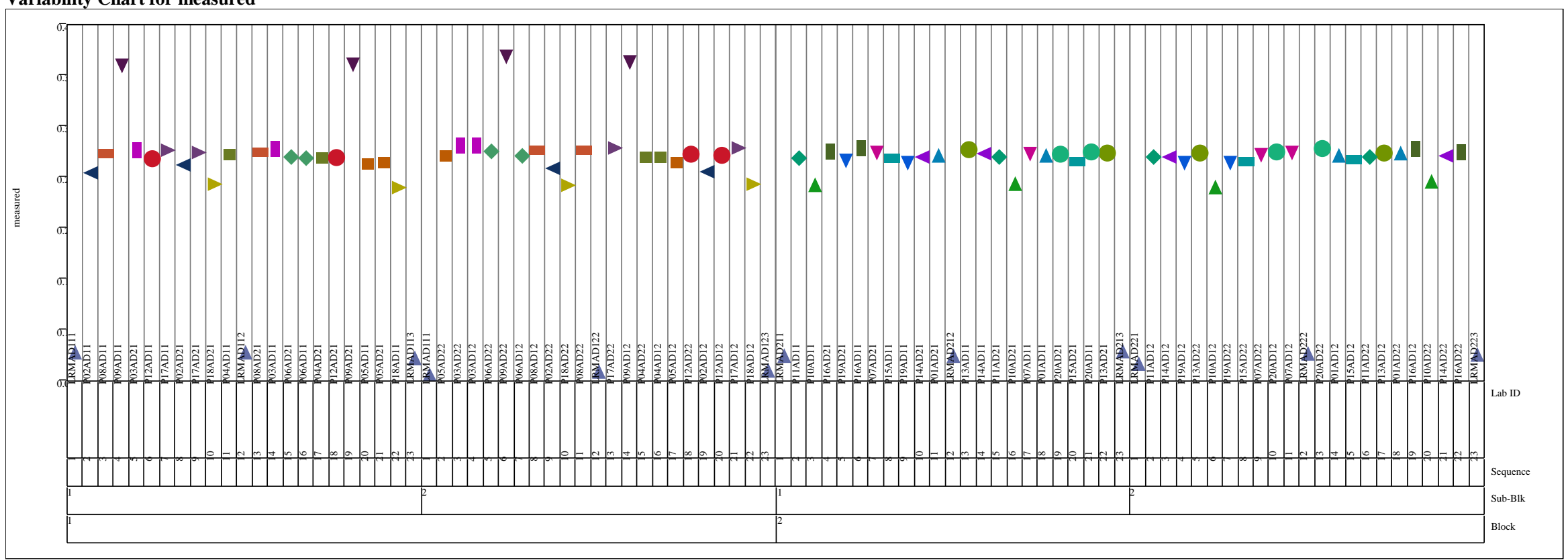


Exhibit A-1. Plots of Oxide Measurements in Analytical Sequence Grouped by Analytical Block (continued)

Oxide=RuO2 (wt\%), Prep Method=AD

Variability Chart for measured

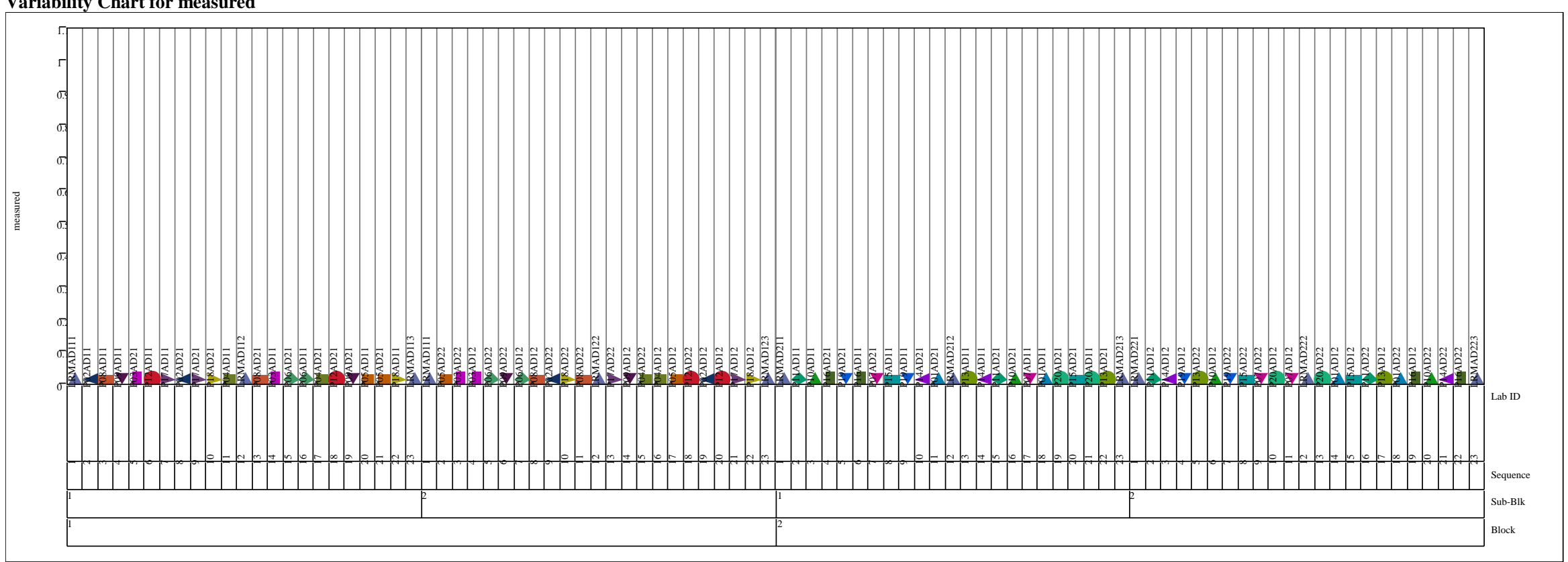




\section{Exhibit A-1. Plots of Oxide Measurements in Analytical Sequence Grouped by Analytical Block (continued)}

Oxide=SiO2 (wt\%), Prep Method=PF

Variability Chart for measured

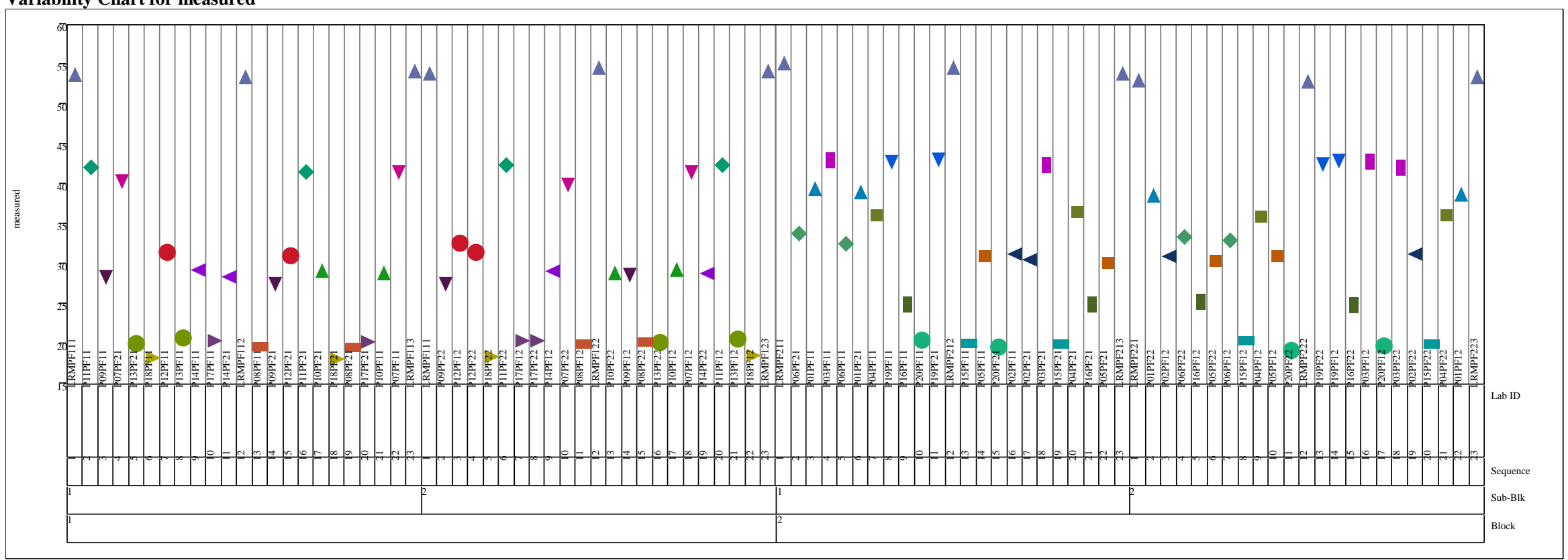




\section{Exhibit A-1. Plots of Oxide Measurements in Analytical Sequence Grouped by Analytical Block (continued)}

Oxide=SO3 (wt \%), Prep Method=AD

Variability Chart for measured

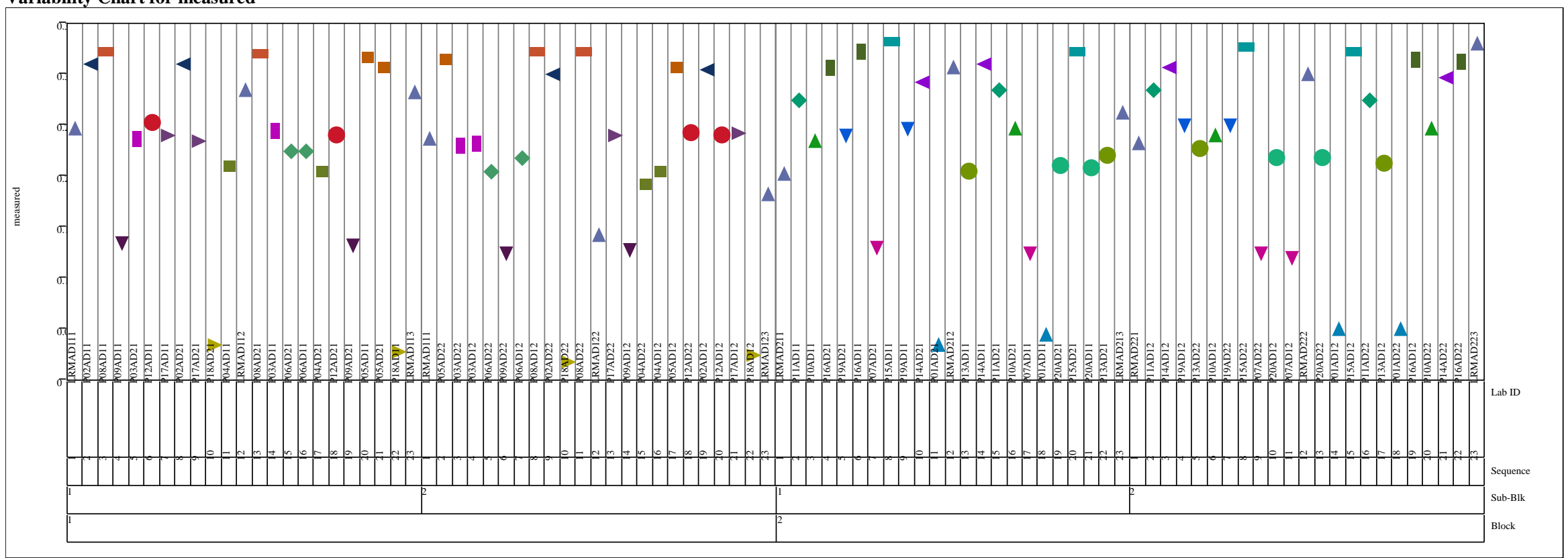


Exhibit A-1. Plots of Oxide Measurements in Analytical Sequence Grouped by Analytical Block (continued)

Oxide=SrO (wt \%), Prep Method $=$ AD

Variability Chart for measured

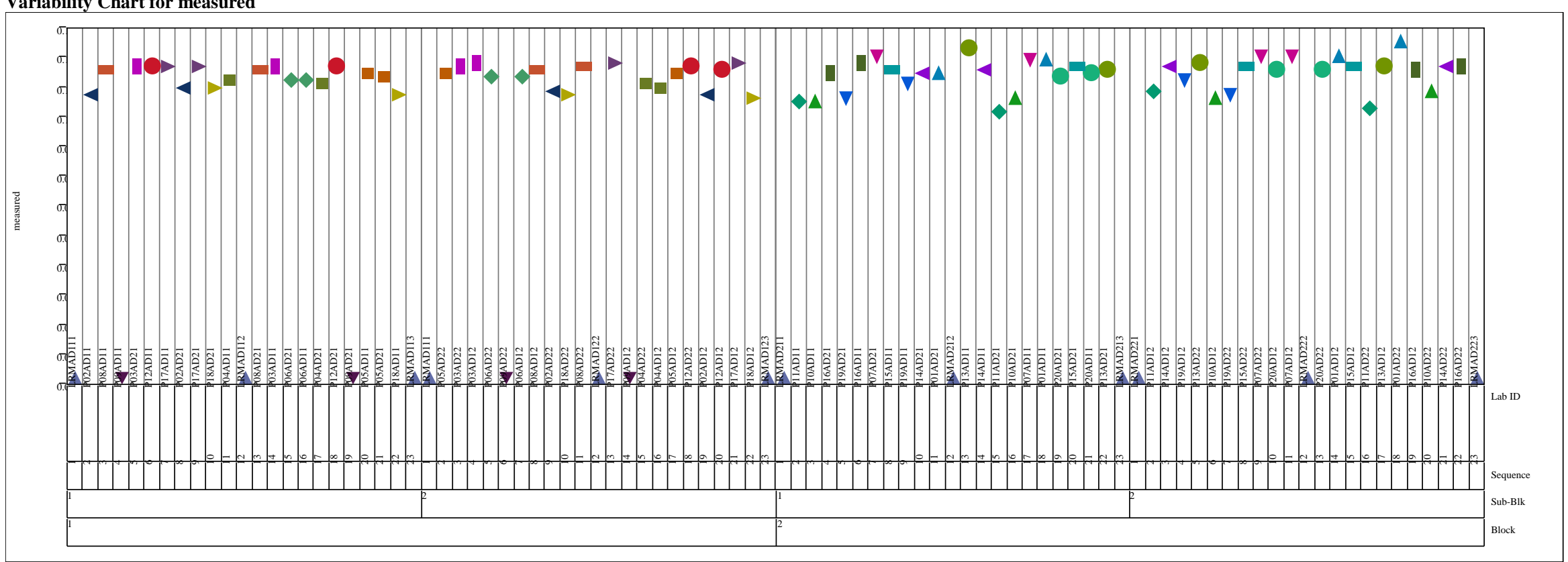




\section{Exhibit A-1. Plots of Oxide Measurements in Analytical Sequence Grouped by Analytical Block (continued)}

Oxide=ZrO2 (wt \%), Prep Method=AD

Variability Chart for measured

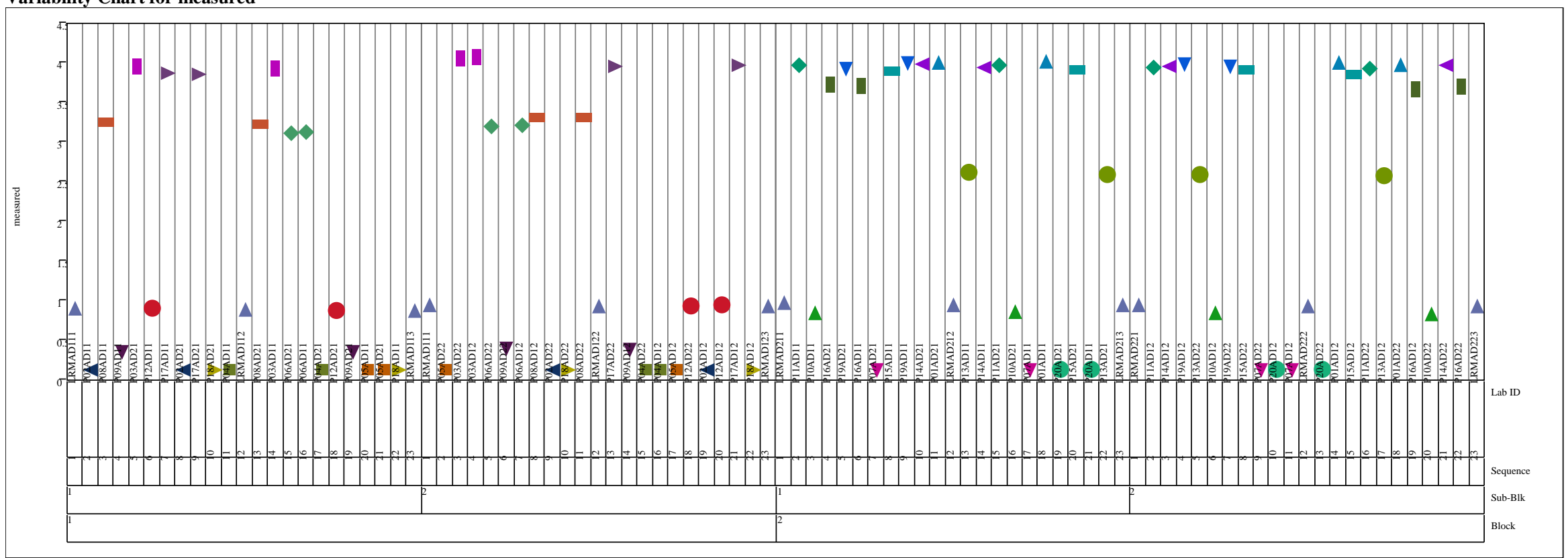




\section{Exhibit A-2. Plots of Oxide Measurements by Glass Identifier Grouped by Targeted Concentrations}

Oxide=Al2O3 (wt\%), Prep Method=PF

Variability Chart for measured

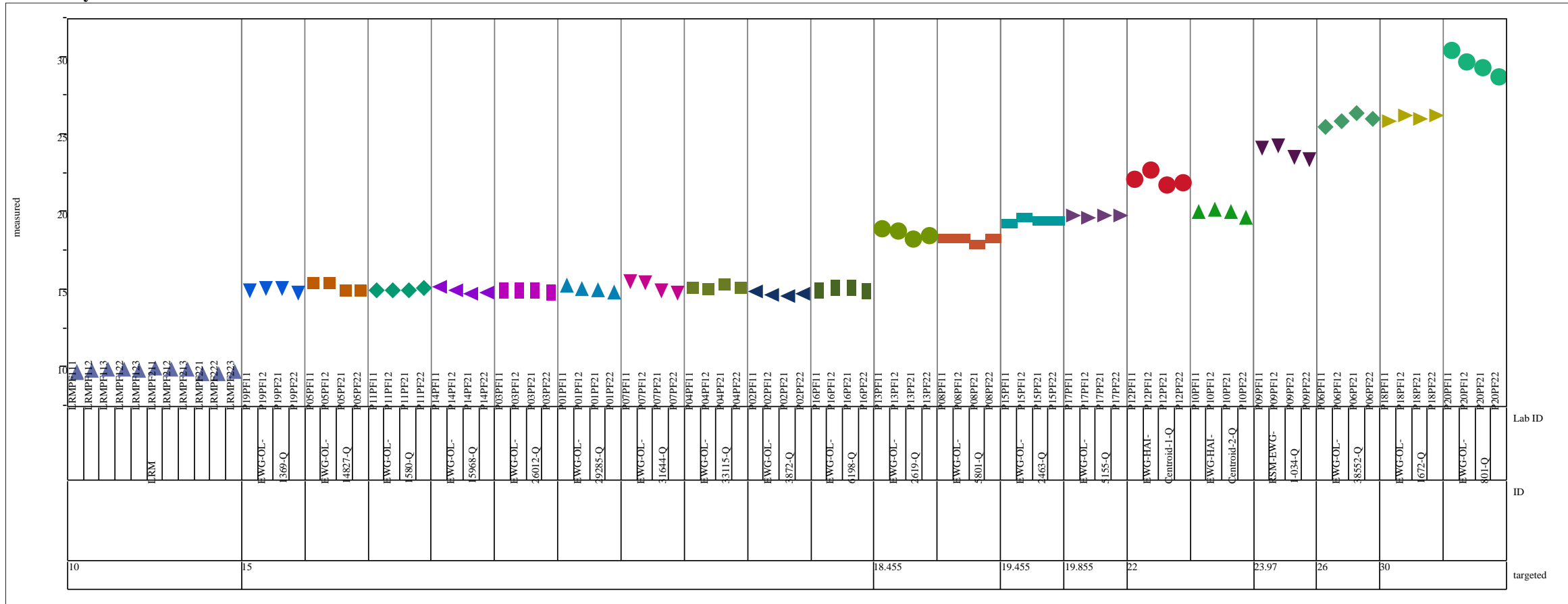


Exhibit A-2. Plots of Oxide Measurements by Glass Identifier Grouped by Targeted Concentrations. (continued)

Oxide=B2O3 (wt \%), Prep Method=PF

Variability Chart for measured

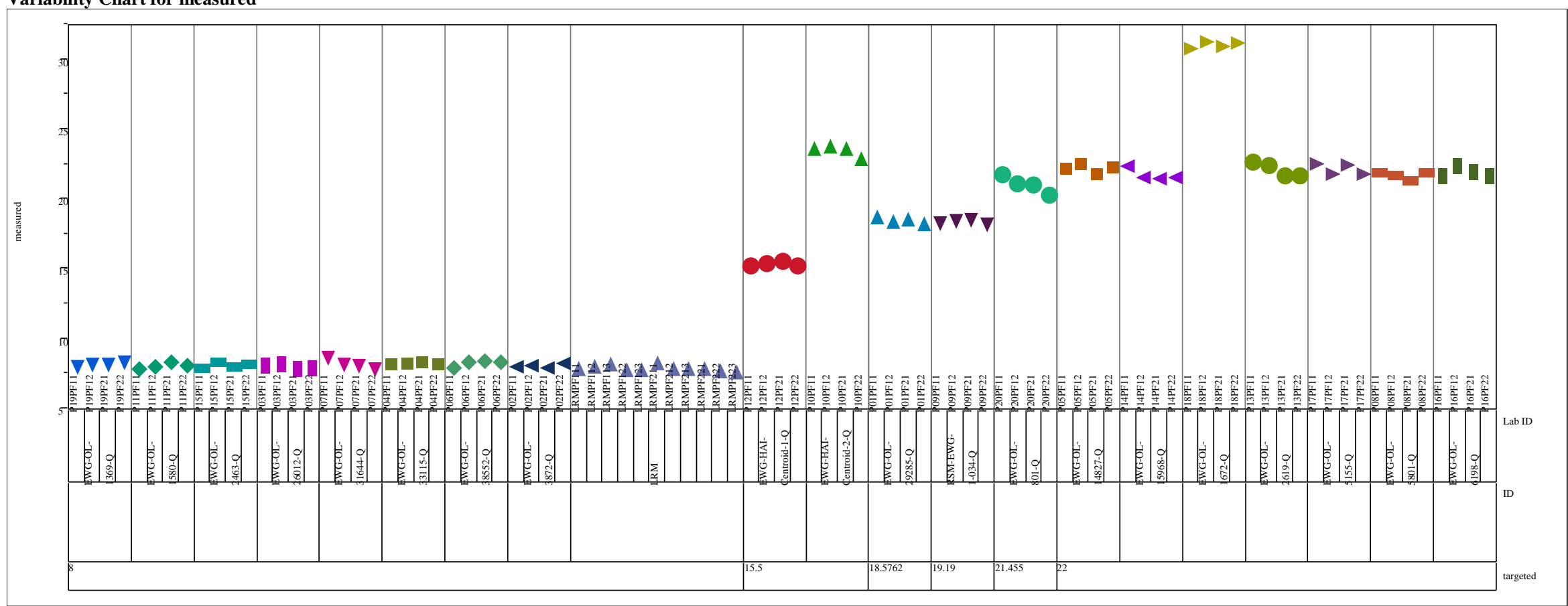


Exhibit A-2. Plots of Oxide Measurements by Glass Identifier Grouped by Targeted Concentrations. (continued)

Oxide=Bi2O3 (wt\%), Prep Method=AD

Variability Chart for measured

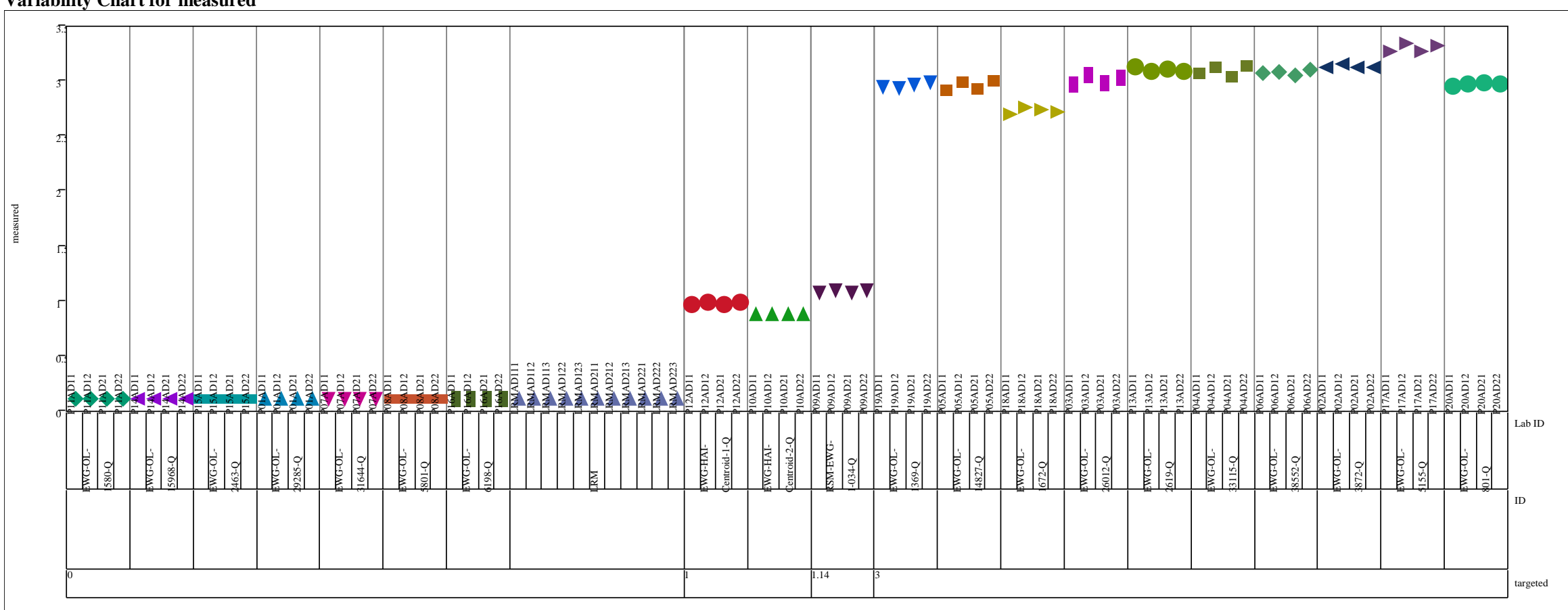


Exhibit A-2. Plots of Oxide Measurements by Glass Identifier Grouped by Targeted Concentrations. (continued)

Oxide $=\mathrm{CaO}(\mathrm{wt} \%)$, Prep Method $=\mathbf{P F}$

Variability Chart for measured

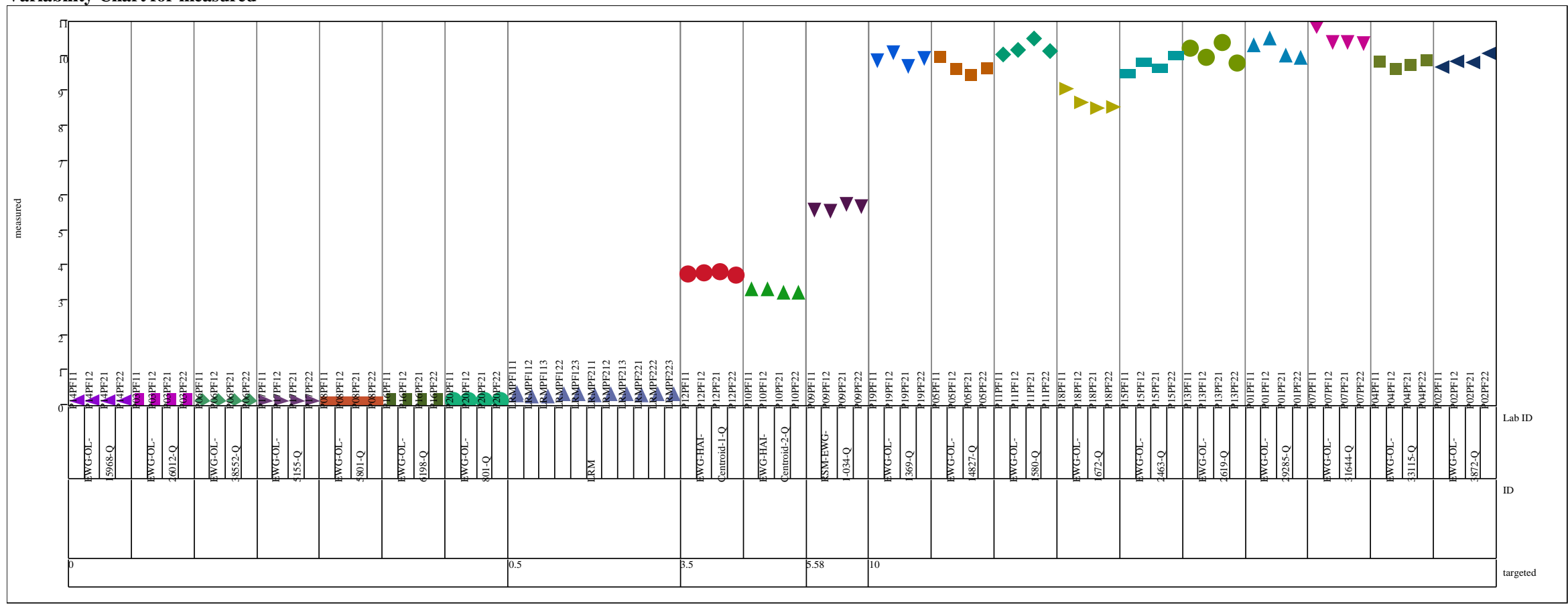


Exhibit A-2. Plots of Oxide Measurements by Glass Identifier Grouped by Targeted Concentrations. (continued)

Oxide $=\mathbf{C d O}($ wt $\%)$, Prep Method $=$ AD

Variability Chart for measured

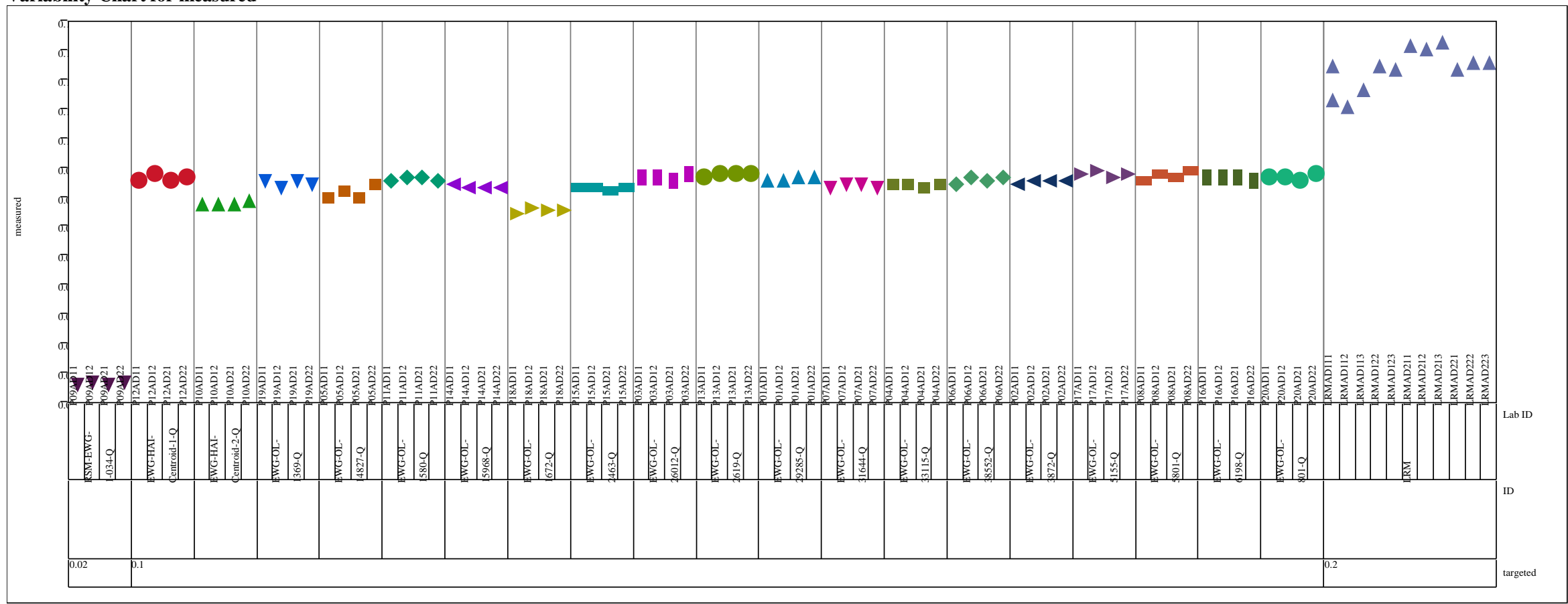


Exhibit A-2. Plots of Oxide Measurements by Glass Identifier Grouped by Targeted Concentrations. (continued)

Oxide $=\mathrm{Cr} 2 \mathrm{O} 3$ (wt \%), Prep Method $=$ PF

Variability Chart for measured

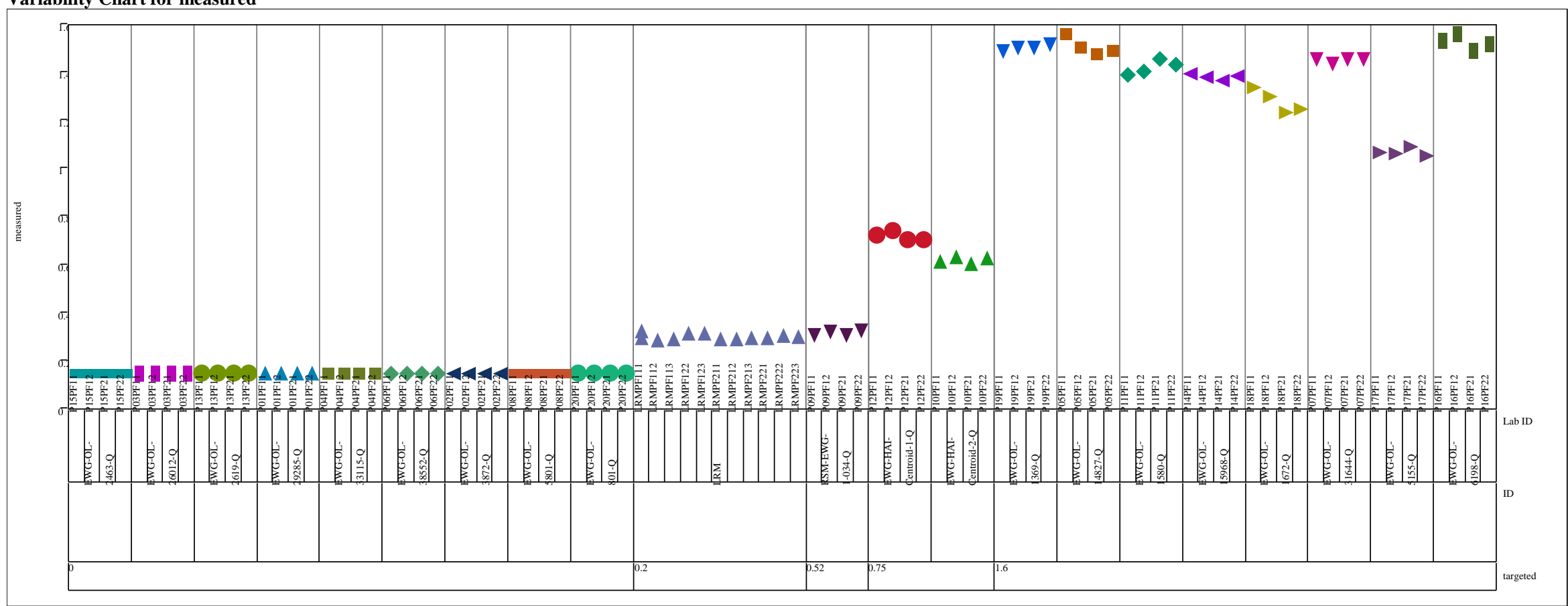


Exhibit A-2. Plots of Oxide Measurements by Glass Identifier Grouped by Targeted Concentrations. (continued) Oxide=Fe2O3 (wt \%), Prep Method=PF Variability Chart for measured

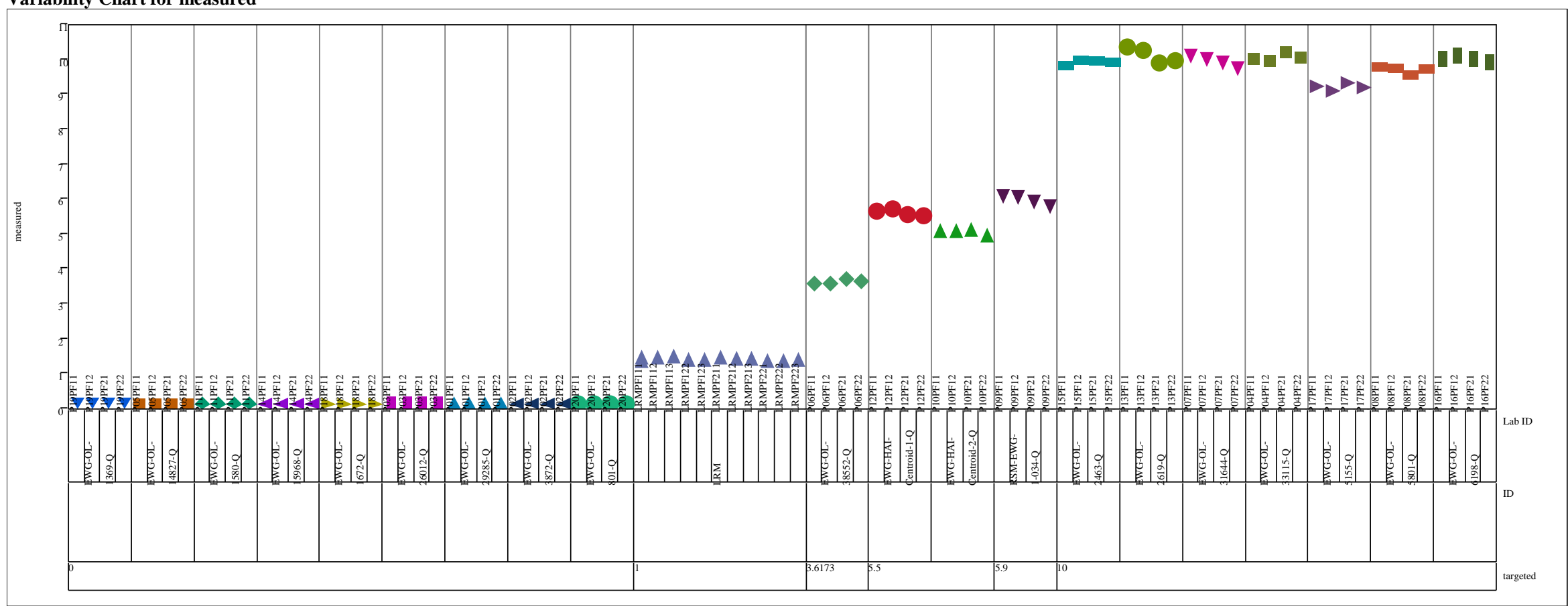


Exhibit A-2. Plots of Oxide Measurements by Glass Identifier Grouped by Targeted Concentrations. (continued)

Oxide=K2O (wt \%), Prep Method=AD

Variability Chart for measured

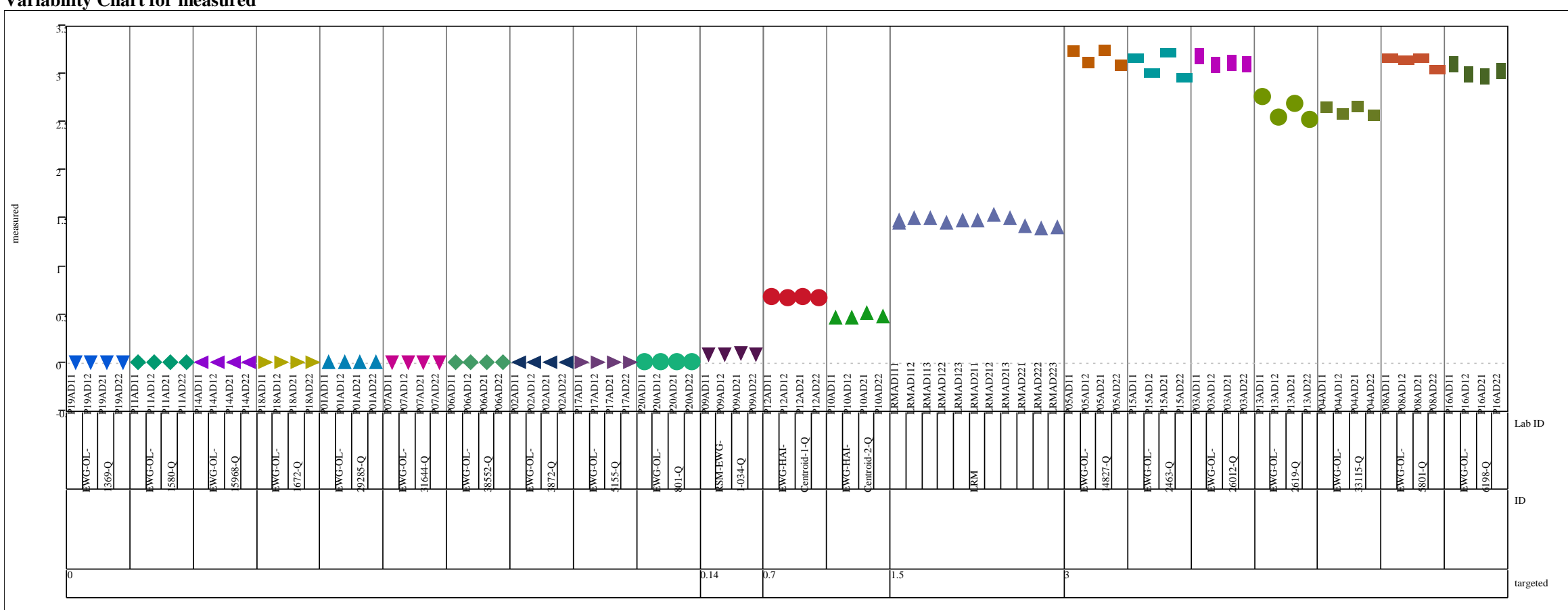


Exhibit A-2. Plots of Oxide Measurements by Glass Identifier Grouped by Targeted Concentrations. (continued)

Oxide=Li2O (wt\%), Prep Method=PF

Variability Chart for measured

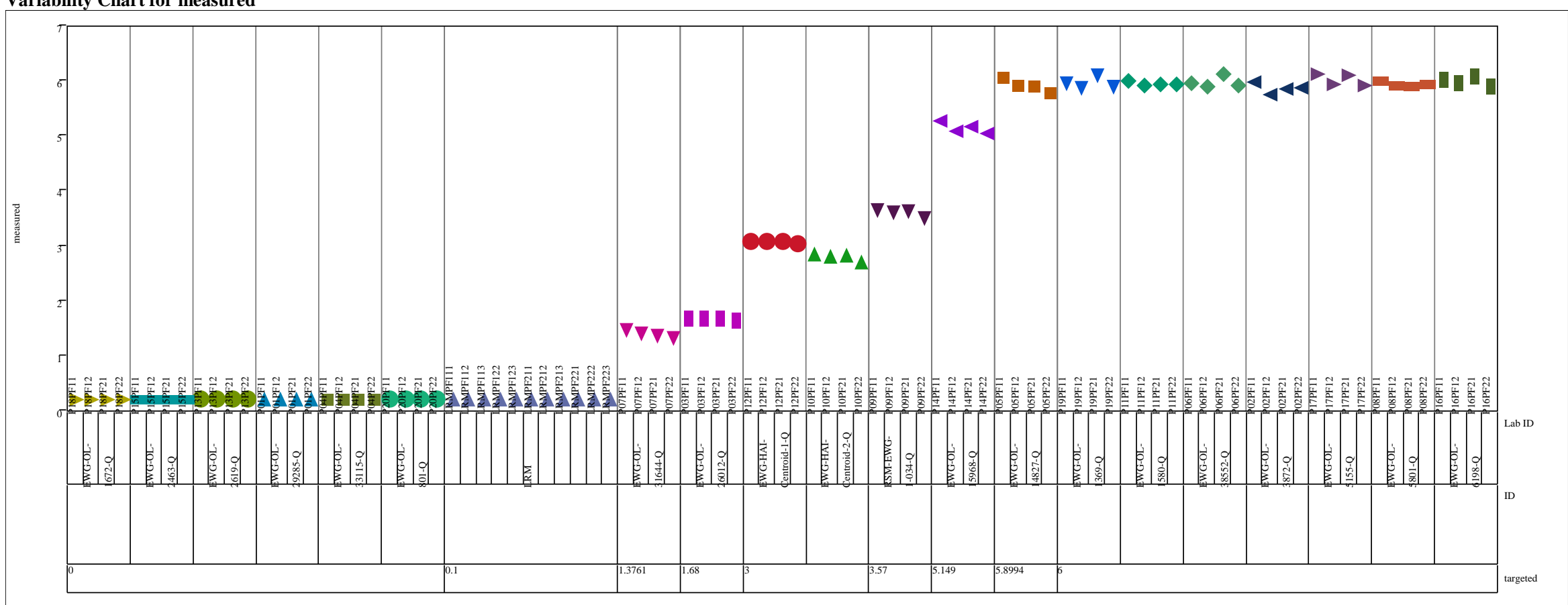


Exhibit A-2. Plots of Oxide Measurements by Glass Identifier Grouped by Targeted Concentrations. (continued)

Oxide=MgO (wt\%), Prep Method $=$ PF

Variability Chart for measured

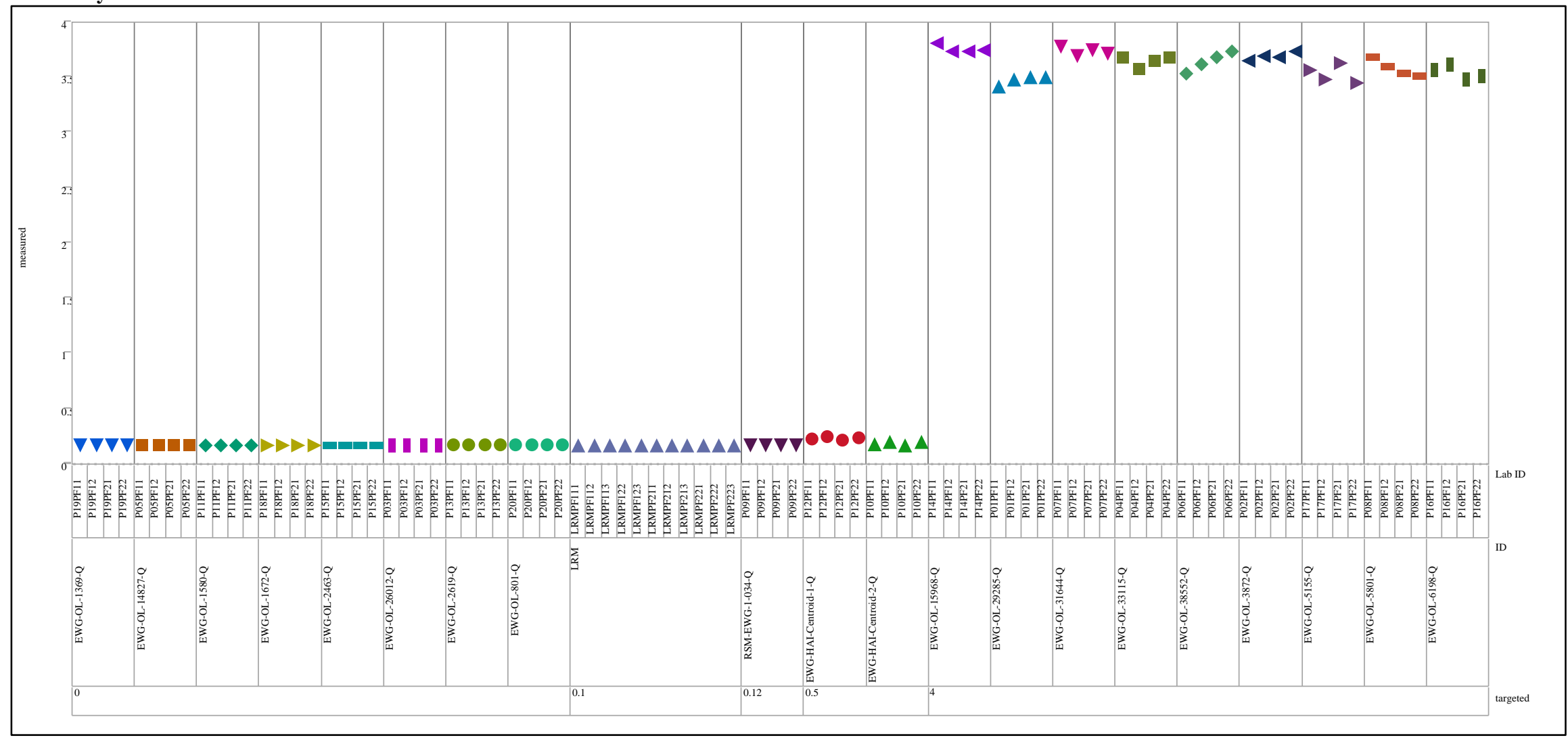


Exhibit A-2. Plots of Oxide Measurements by Glass Identifier Grouped by Targeted Concentrations. (continued) Oxide=MnO (wt\%), Prep Method=PF Variability Chart for measured

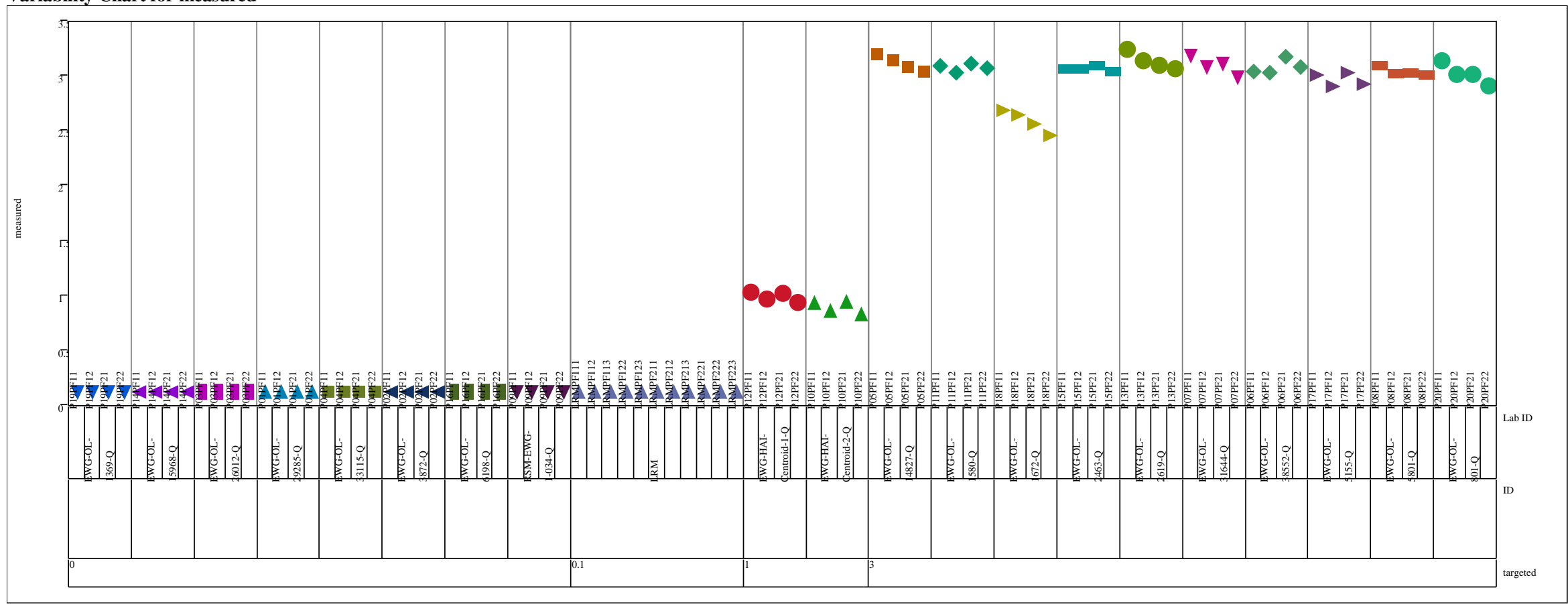


Exhibit A-2. Plots of Oxide Measurements by Glass Identifier Grouped by Targeted Concentrations. (continued)

Oxide=Na2O (wt \%), Prep Method $=$ AD

Variability Chart for measured

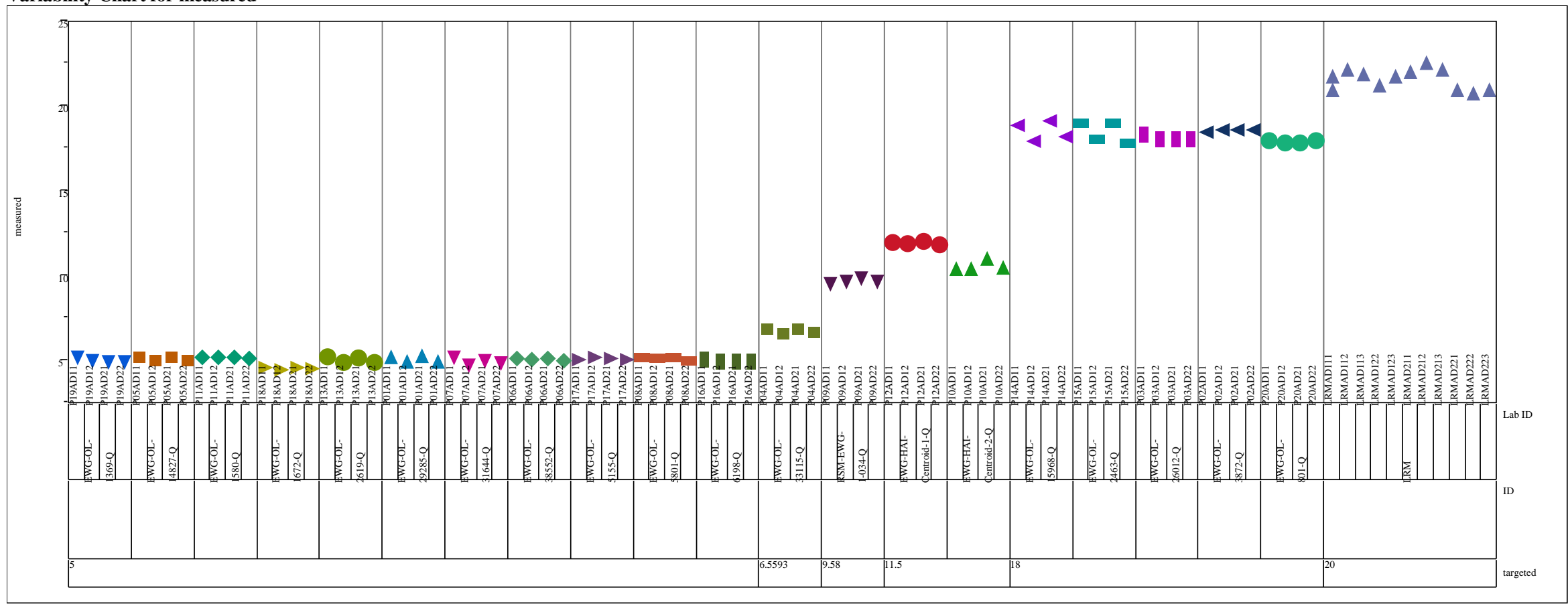


Exhibit A-2. Plots of Oxide Measurements by Glass Identifier Grouped by Targeted Concentrations. (continued)

Oxide=NiO (wt \%), Prep Method=AD

Variability Chart for measured

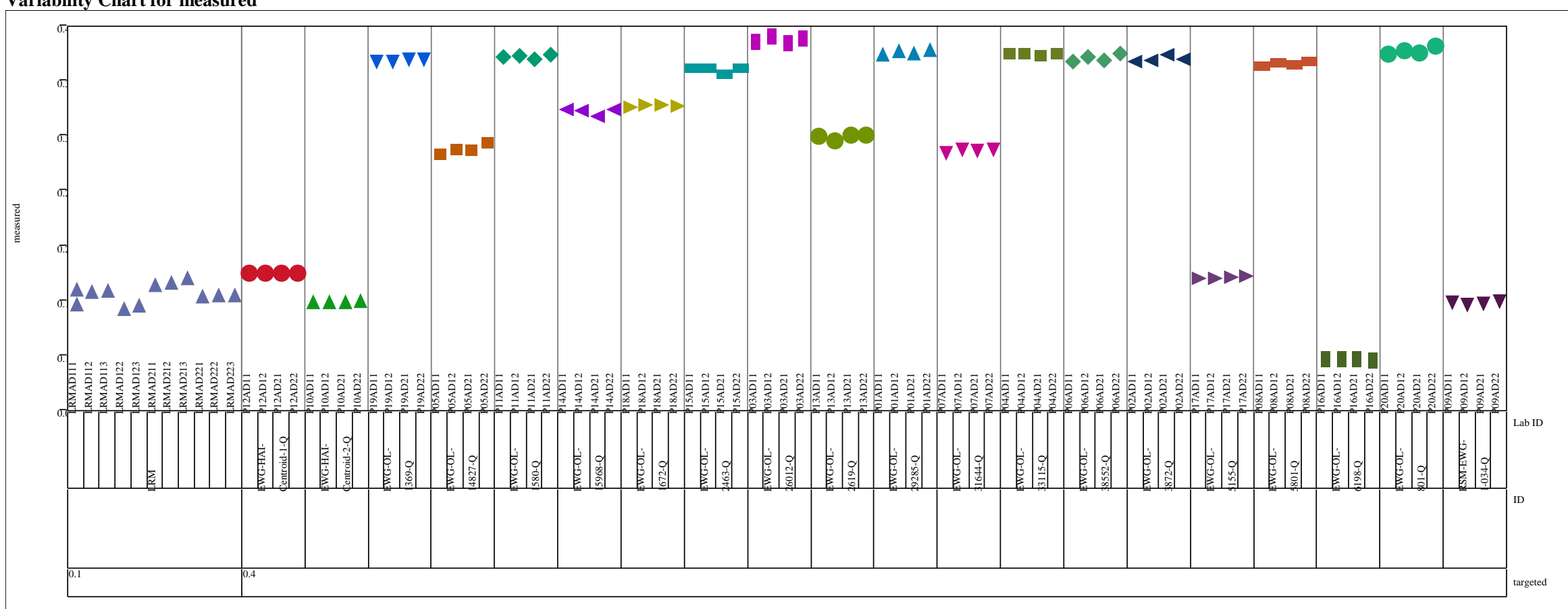


Exhibit A-2. Plots of Oxide Measurements by Glass Identifier Grouped by Targeted Concentrations. (continued)

Oxide $=$ P2O5 (wt \%), Prep Method=AD

Variability Chart for measured

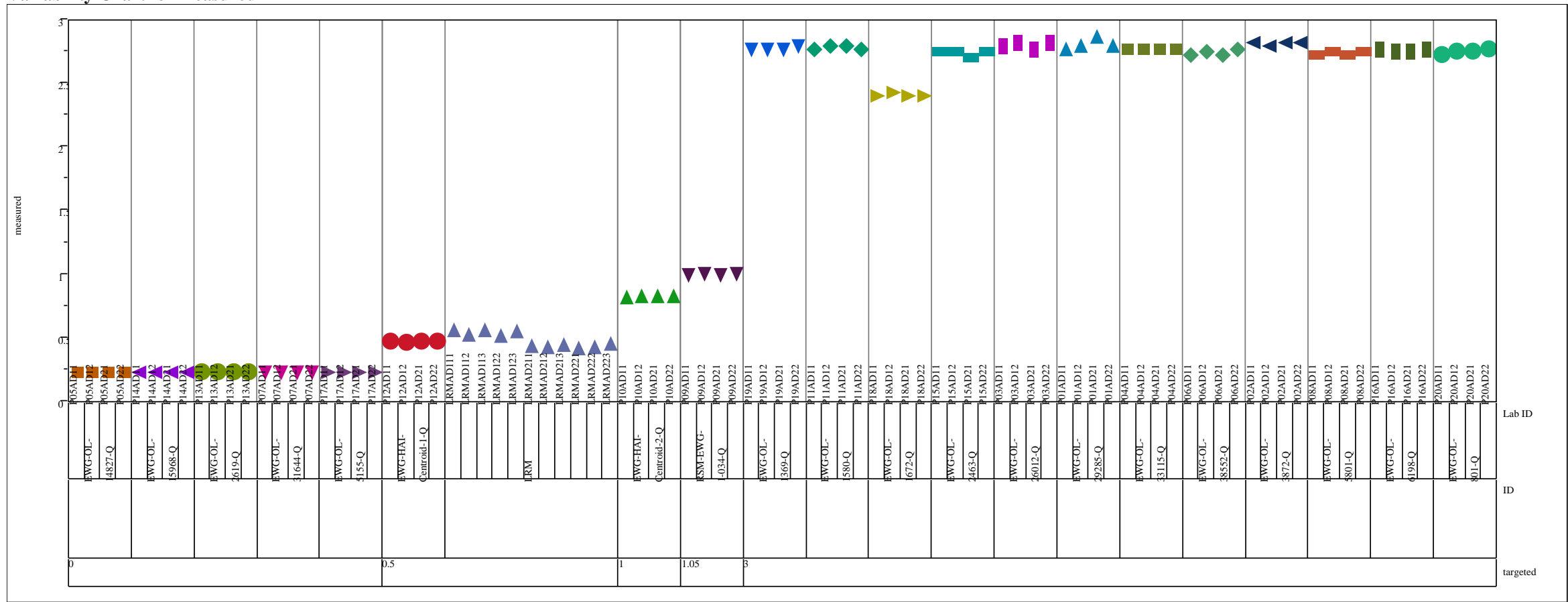


Exhibit A-2. Plots of Oxide Measurements by Glass Identifier Grouped by Targeted Concentrations. (continued)

Oxide $=$ PbO (wt \%), Prep Method=AD

Variability Chart for measured

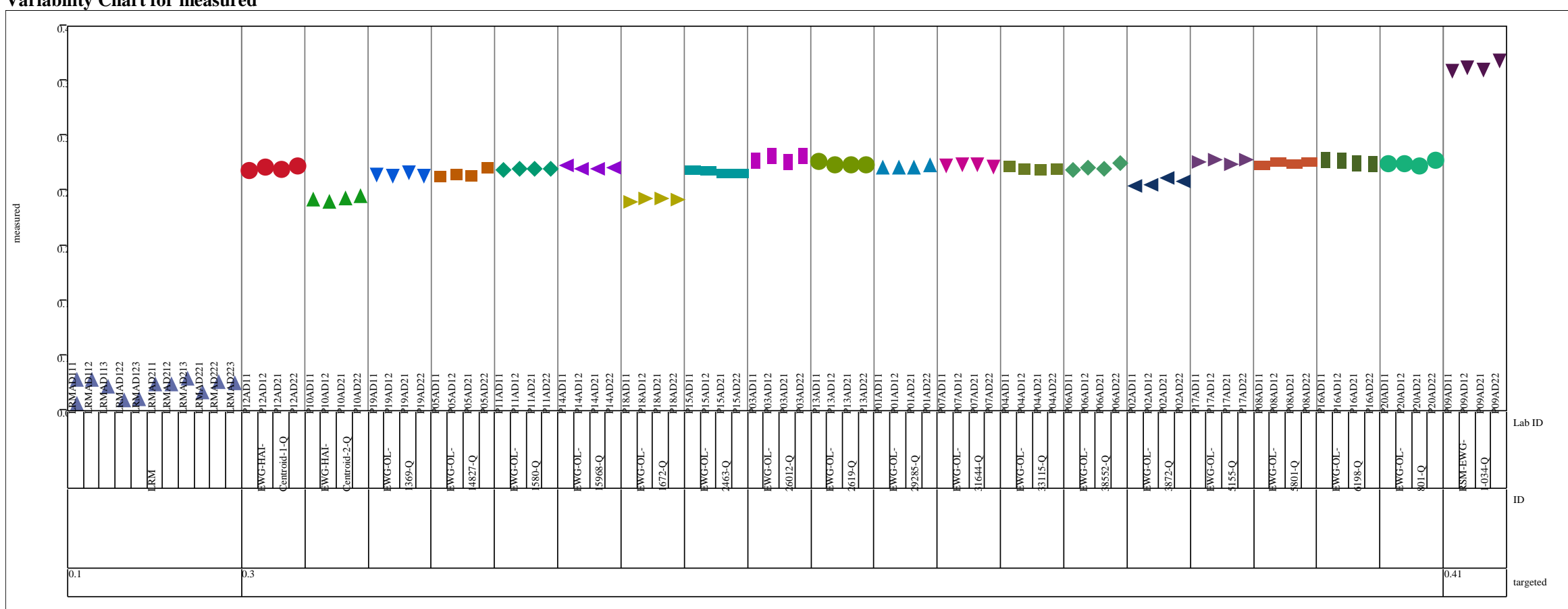


Exhibit A-2. Plots of Oxide Measurements by Glass Identifier Grouped by Targeted Concentrations. (continued)

Oxide=SiO2 (wt\%), Prep Method=PF

Variability Chart for measured

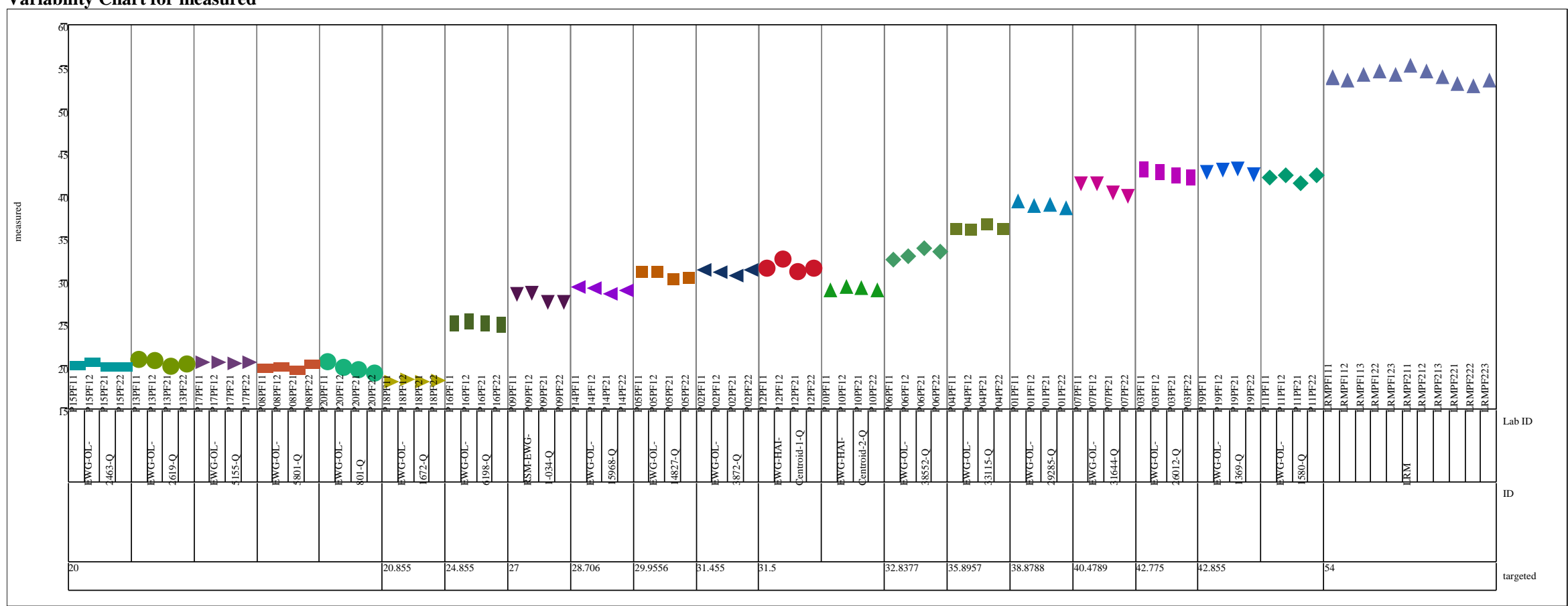


Exhibit A-2. Plots of Oxide Measurements by Glass Identifier Grouped by Targeted Concentrations. (continued)

Oxide=SO3 (wt \%), Prep Method=AD

Variability Chart for measured

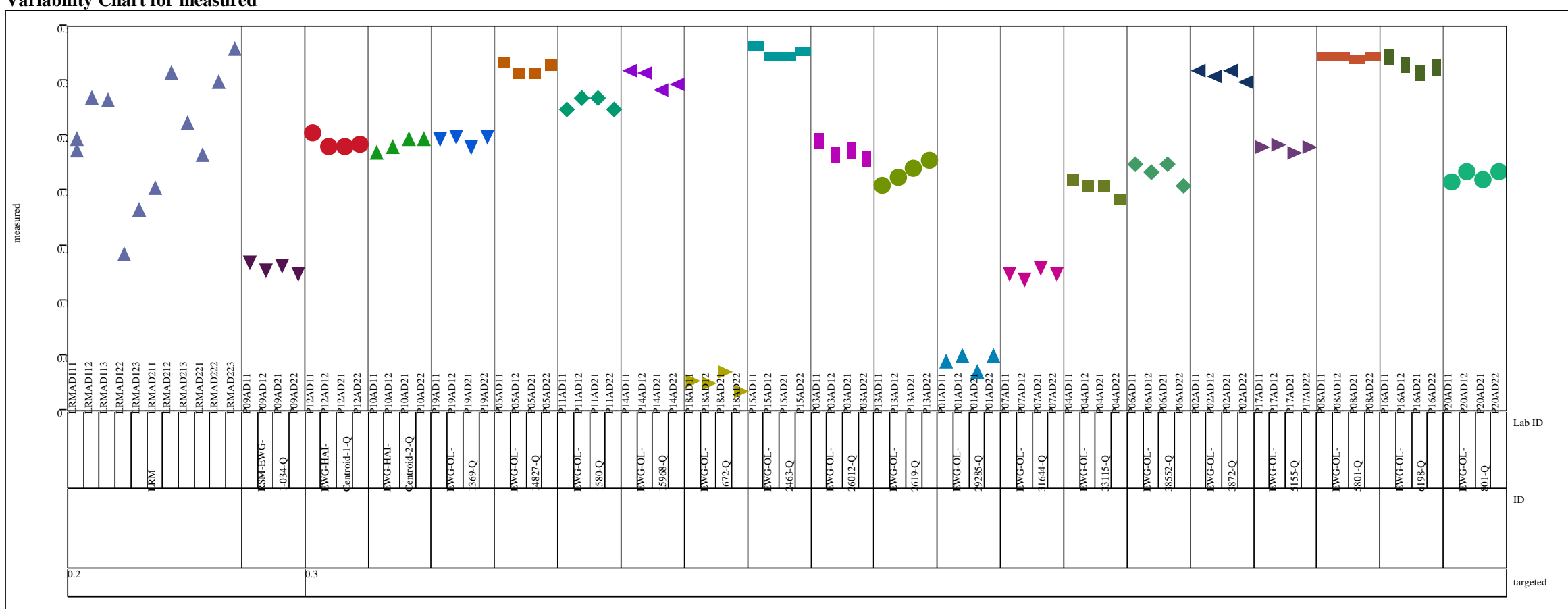


Exhibit A-2. Plots of Oxide Measurements by Glass Identifier Grouped by Targeted Concentrations. (continued)

Oxide $=$ SrO (wt \%), Prep Method $=$ AD

Variability Chart for measured

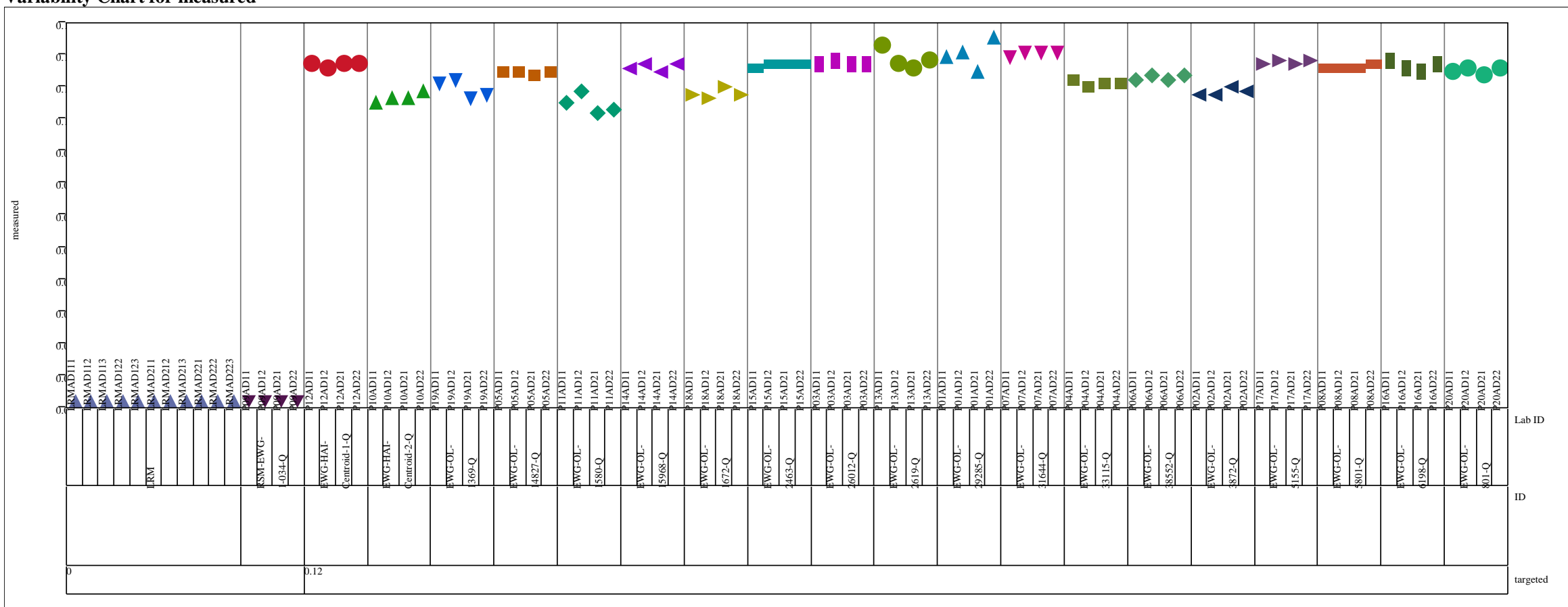


Exhibit A-2. Plots of Oxide Measurements by Glass Identifier Grouped by Targeted Concentrations. (continued)

Oxide=ZrO2 (wt\%), Prep Method=AD

Variability Chart for measured

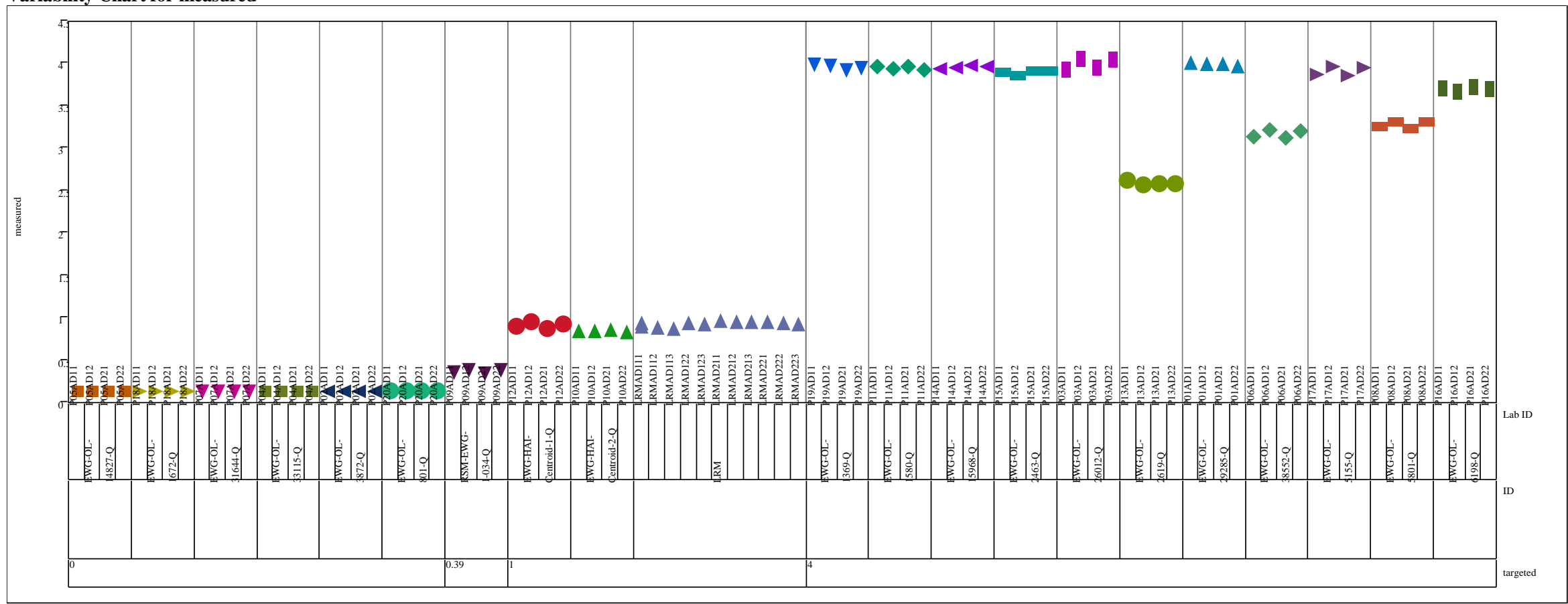


Exhibit A-3. Analysis of Variance of Analytical Block Effects for Measurements of the LRM Standard Glass

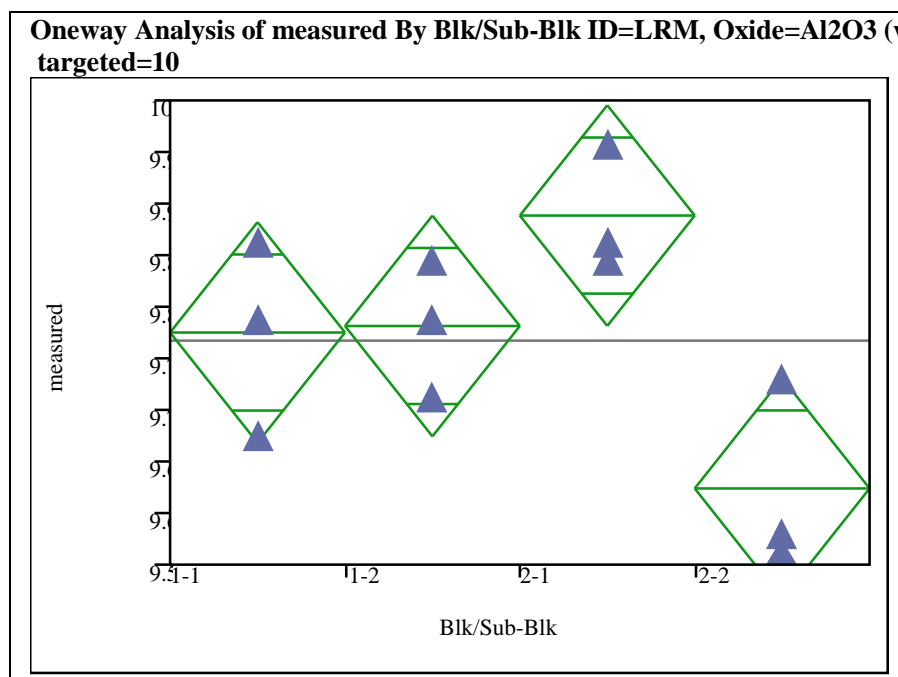

\section{Oneway Anova}

Summary of Fit

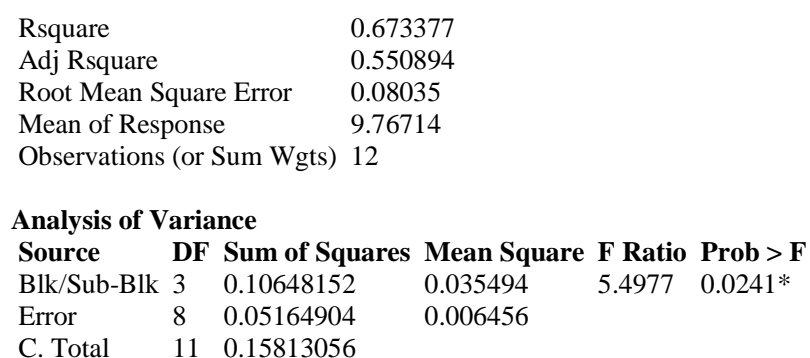

Observations (or Sum Wgts) 12

Analysis of Variance

\begin{tabular}{lllllll} 
Analysis of & \multicolumn{2}{l}{$\begin{array}{llll}\text { Variance } \\
\text { Source }\end{array}$} & DF & Sum of Squares & Mean Square & F Ratio Prob > F \\
Blk/Sub-Blk & 3 & 0.10648152 & 0.035494 & 5.4977 & $0.0241^{*}$ \\
Error & 8 & 0.05164904 & 0.006456 & & \\
C. Total & 11 & 0.15813056 & & &
\end{tabular}

\section{Means for Oneway Anova}

Level Number Mean Std Error Lower 95\% Upper 95\%
$1-1 \quad 3$
$\begin{array}{llll}\text { Mean } & \text { Std Error } & \text { Lower } 95 \% & \text { Upper } 95 \% \\ 9.77501 & 0.04639 & 9.6680 & 9.8820 \\ 9.78131 & 0.04639 & 9.6743 & 9.8883\end{array}$

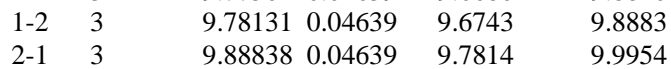

$\begin{array}{llllll}2-2 & 3 & 9.62385 & 0.04639 & 9.5169 & 9.7308\end{array}$

Std Error uses a pooled estimate of error variance

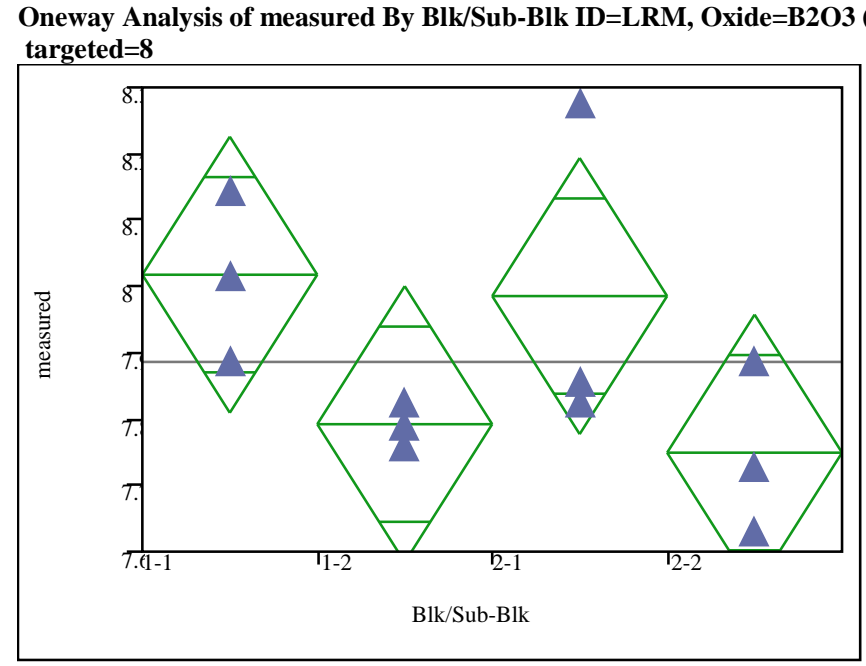

Oneway Anova

Summary of Fit

$\begin{array}{ll}\text { Adj Rsquare } & 0.252222 \\ \text { Root Mean Square Error } & 0.156367\end{array}$

Mean of Response $\quad 7.886072$

Observations (or Sum Wgts) 12

Analysis of Variance

$\begin{array}{llllll}\text { Source } & \text { DF } & \text { Sum of Squares } & \text { Mean Square } & \text { F Ratio } & \text { Prob }>\text { F } \\ \text { Blk/Sub-Blk } & 3 & 0.16406974 & 0.054690 & 2.2367 & 0.1613\end{array}$

$\begin{array}{llll}\text { Blk/Sub-Blk } & 3 & 0.16406974 & 0.054690 \\ \text { Error } & 8 & 0.19560500 & 0.024451\end{array}$

C. Total $11 \quad 0.35967474$

\section{Means for Oneway Anova}

Level Number Mean Std Error Lower 95\% Upper 95\%
$1-1$
$\begin{array}{lll}8.01755 & 0.09028 & 7.8094\end{array}$
8.2257
$1-2 \quad 3$
$\begin{array}{lll}7.79216 & 0.09028 & 7.5840\end{array}$
8.0003
$\begin{array}{llllll}2-1 & 3 & 7.98535 & 0.09028 & 7.7772 & 8.1935\end{array}$
$\begin{array}{llllll}2-2 & 3 & 7.74923 & 0.09028 & 7.5410 & 7.9574\end{array}$

Std Error uses a pooled estimate of error variance 
Exhibit A-3. Analysis of Variance of Analytical Block Effects for Measurements of the LRM Standard Glass (continued)

Oneway Analysis of measured By Blk/Sub-Blk ID=LRM, Oxide=Bi2O3 (wt\%), targeted $=0$

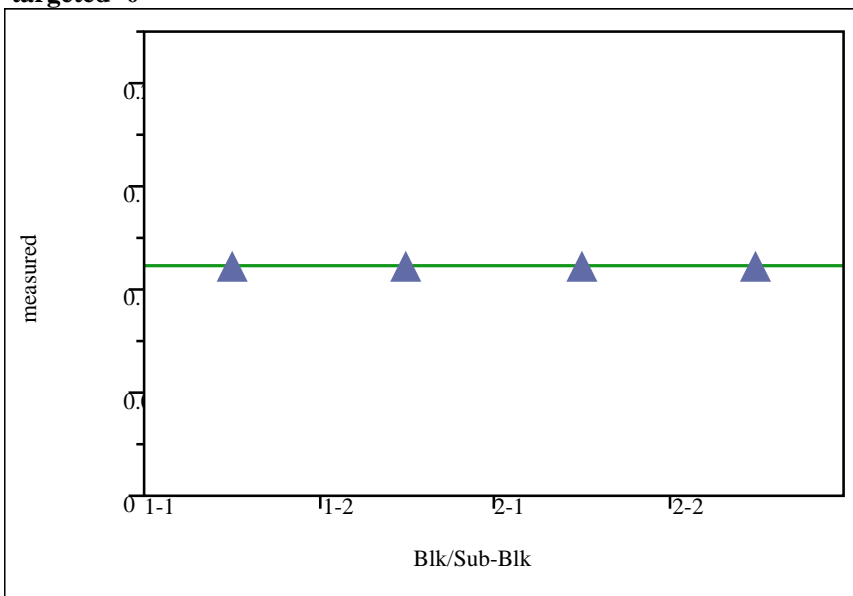

Oneway Anova

Summary of Fit

Rsquare

Adj Rsquare

Root Mean Square Error

Mean of Response

0.11148

Observations (or Sum Wgts) 12

\section{Analysis of Variance}

Source DF Sum of Squares Mean Square F Ratio Prob $>$ F

Blk/Sub-Blk 3 0

$\begin{array}{llll}\text { Error } & 8 & 0 & 0\end{array}$

C. Total $11 \quad 0$

Means for Oneway Anova

Level Number Mean Std Error Lower 95\% Upper 95\%

$\begin{array}{llllll}1-1 & 3 & 0.111480 & 0 & 0.11148 & 0.11148\end{array}$

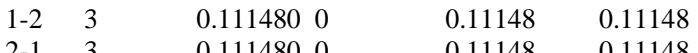

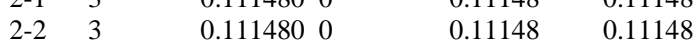

Std Error uses a pooled estimate of error variance
Oneway Analysis of measured By Blk/Sub-Blk ID=LRM, Oxide=CaO (wt\%), targeted $=0.5$

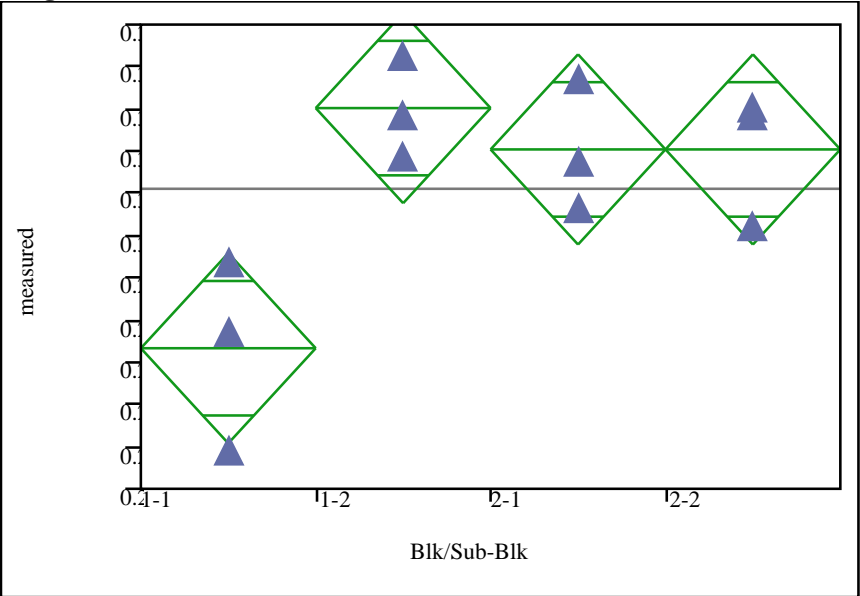

Oneway Anova

Summary of Fit

$\begin{array}{ll}\text { Rsquare } & 0.720249 \\ \text { Adj Rsquare } & 0.615342 \\ \text { Root Mean Square Error } & 0.016926 \\ \text { Mean of Response } & 0.311089 \\ \text { Observations (or Sum Wgts) } & 12\end{array}$

Analysis of Variance

Source DF Sum of Squares Mean Square F Ratio Prob $>$ F

$\begin{array}{lllll}\text { Blk/Sub-Blk } 3 & 0.00590069 & 0.001967 & 6.8656 & 0.0133 *\end{array}$

$\begin{array}{llll}\text { Error } & 8 & 0.00229189 & 0.000286\end{array}$

C. Total $11 \quad 0.00819258$

Means for Oneway Anova

Level Number Mean Std Error Lower 95\% Upper 95\%

$\begin{array}{llllll}1-1 & 3 & 0.273310 & 0.00977 & 0.25078 & 0.29585\end{array}$

$\begin{array}{llllll}1-2 & 3 & 0.330211 & 0.00977 & 0.30768 & 0.35275\end{array}$

$\begin{array}{llllll}2-1 & 3 & 0.320417 & 0.00977 & 0.29788 & 0.34295\end{array}$

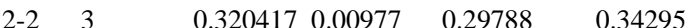

Std Error uses a pooled estimate of error variance 
Exhibit A-3. Analysis of Variance of Analytical Block Effects for Measurements of the LRM Standard Glass (continued)

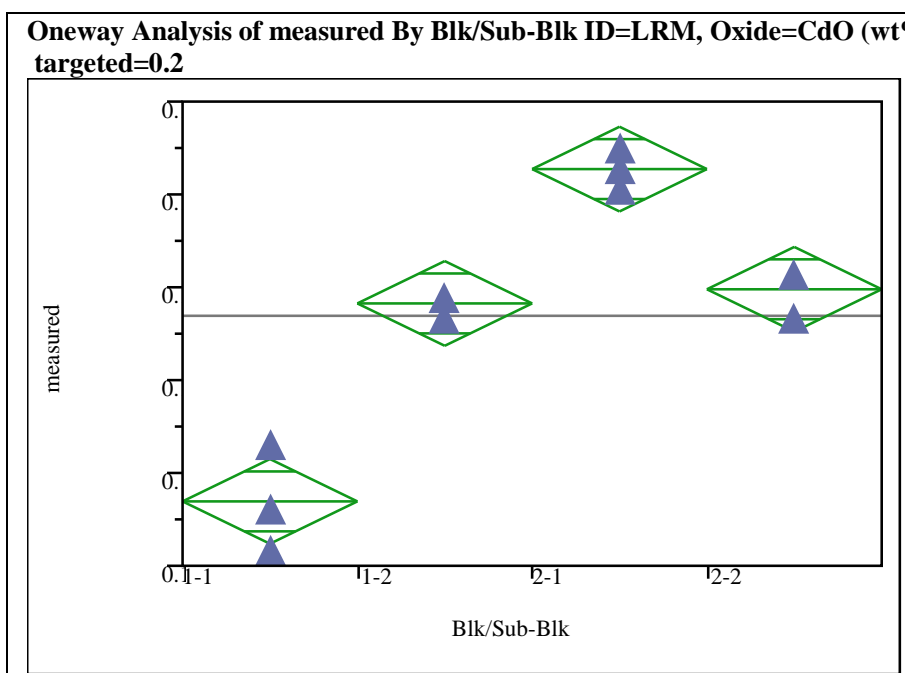

Oneway Anova

Summary of Fit

$\begin{array}{lr}\text { Rsquare } & 0.95465 \\ \text { Adj Rsquare } & 0.937644 \\ \text { Root Mean Square Error } & 0.001713 \\ \text { Mean of Response } & 0.123464 \\ \text { Observations (or Sum Wgts) } & 12\end{array}$

Analysis of Variance

Source DF Sum of Squares Mean Square F Ratio Prob $>$ F

Blk/Sub-Blk $3 \quad 0.00049443 \quad 0.00016556 .1358<.0001^{*}$

$\begin{array}{llll}\text { Error } & 8 & 0.00002349 & 2.936 \mathrm{e}-6\end{array}$

C. Total $\quad 11 \quad 0.00051792$

Oneway Analysis of measured By Blk/Sub-Blk ID=LRM, Oxide=Cr2O3 (wt\%), targeted $=0.2$

Means for Oneway Anova

Level Number Mean Std Error Lower 95\% Upper 95\%

$\begin{array}{llllll}1-1 & 3 & 0.113468 & 0.00099 & 0.11119 & 0.11575\end{array}$

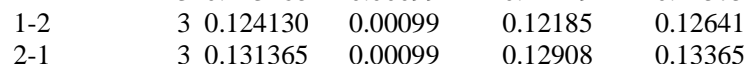

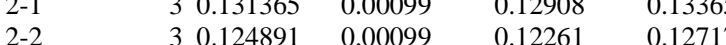

Std Error uses a pooled estimate of error variance

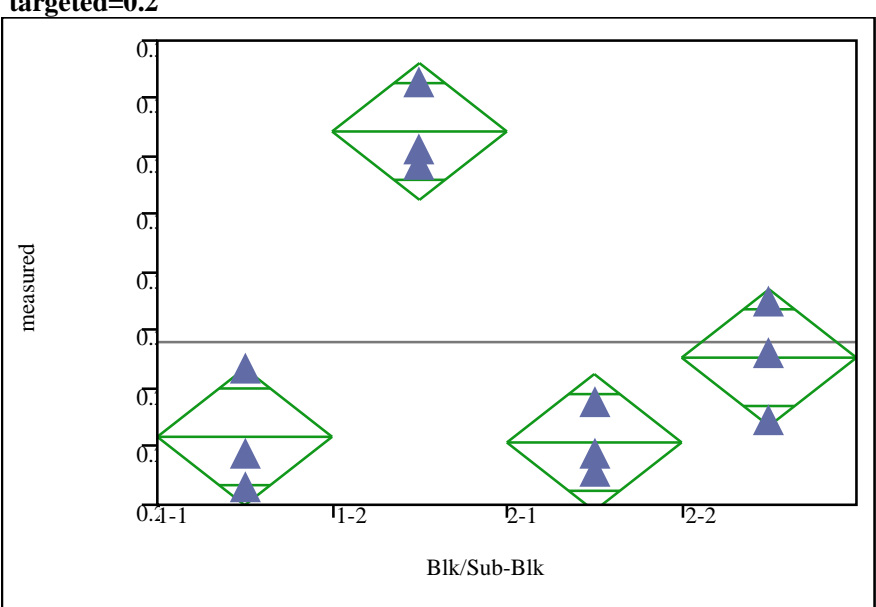

Oneway Anova

Summary of Fit

$\begin{array}{lr}\text { Rsquare } & 0.900486 \\ \text { Adj Rsquare } & 0.863169 \\ \text { Root Mean Square Error } & 0.004425 \\ \text { Mean of Response } & 0.299019 \\ \text { Observations (or Sum Wgts) } & 12\end{array}$

Analysis of Variance

Source DF Sum of Squares Mean Square F Ratio Prob $>$ F

$\begin{array}{llllll}\text { Blk/Sub-Blk } & 3 & 0.00141760 & 0.00047324 .1303 & 0.0002 *\end{array}$

$\begin{array}{llll}\text { Error } & 8 & 0.00015666 & 0.000020\end{array}$

$\begin{array}{lll}\text { C. Total } & 11 & 0.00157426\end{array}$

Means for Oneway Anova

Level Number Mean Std Error Lower 95\% Upper 95\%

$\begin{array}{lllllr}1-1 & 3 & 0.290858 & 0.00255 & 0.28497 & 0.29675\end{array}$

$\begin{array}{llllll}1-2 & 3 & 0.317167 & 0.00255 & 0.31128 & 0.32306\end{array}$

$\begin{array}{llllll}2-1 & 3 & 0.290371 & 0.00255 & 0.28448 & 0.29626\end{array}$

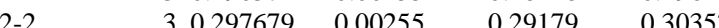

Std Error uses a pooled estimate of error variance 
Exhibit A-3. Analysis of Variance of Analytical Block Effects for Measurements of the LRM Standard Glass (continued)

Oneway Analysis of measured By Blk/Sub-Blk ID=LRM, Oxide=Fe2O3 (wt\%), targeted $=1$

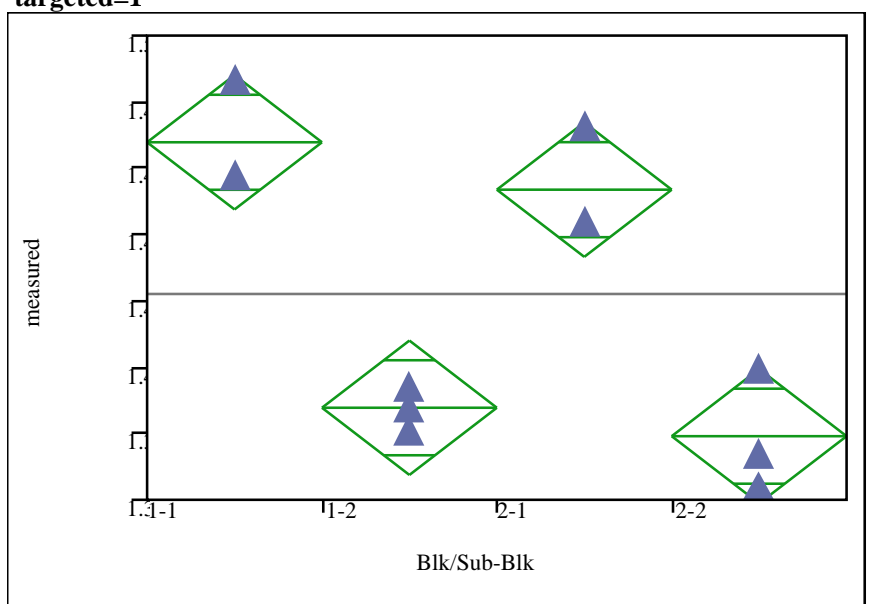

Oneway Anova

Summary of Fit
Rsquare
0.908034
Adj Rsquare
0.873546
Root Mean Square Error 0.01522
Mean of Response 1.422075
Observations (or Sum Wgts) 12
Analysis of Variance
Source DF Sum of Squares Mean Square F Ratio Prob $>$ F
Blk/Sub-Blk $3 \quad 0.01829826 \quad 0.00609926 .3294 \quad 0.0002 *$
$\begin{array}{llll}\text { Error } & 8 & 0.00185326 & 0.000232\end{array}$
$\begin{array}{lll}\text { C. Total } & 11 & 0.02015153\end{array}$

Means for Oneway Anova

Level Number Mean Std Error Lower 95\% Upper 95\%

$\begin{array}{lllrrr}1-1 & 3 & 1.46783 & 0.00879 & 1.4476 & 1.4881 \\ 1-2 & 3 & 1.38776 & 0.00879 & 1.3675 & 1.4080\end{array}$

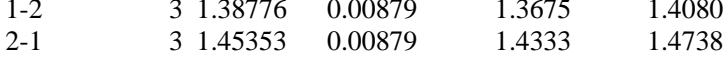

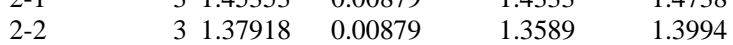

Std Error uses a pooled estimate of error variance
Oneway Analysis of measured By Blk/Sub-Blk ID=LRM, Oxide=K2O (wt\%), targeted $=1.5$

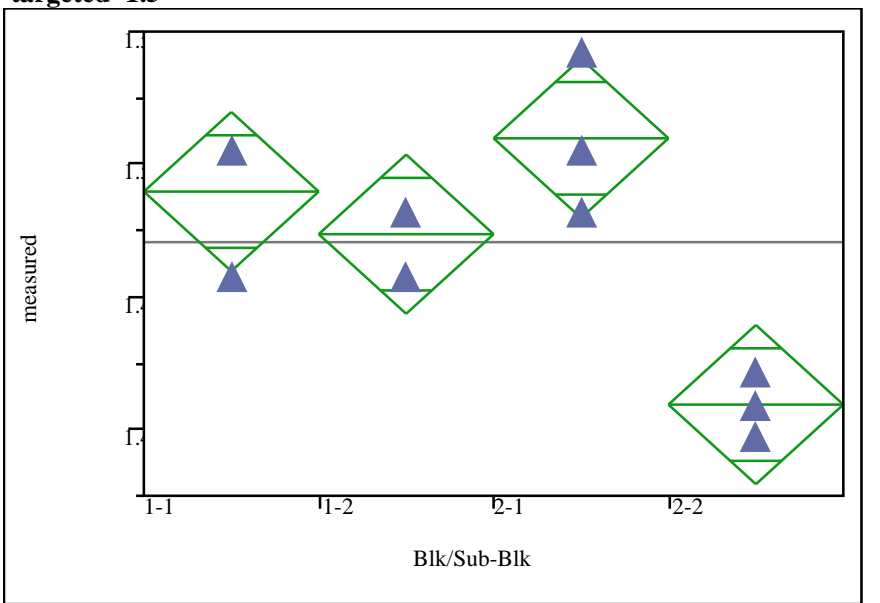

Oneway Anova

Summary of Fit

$\begin{array}{lr}\text { Rsquare } & 0.806786 \\ \text { Adj Rsquare } & 0.73433 \\ \text { Root Mean Square Error } & 0.022536 \\ \text { Mean of Response } & 1.470616 \\ \text { Observations (or Sum Wgts) } & 12\end{array}$

Analysis of Variance

Source DF Sum of Squares Mean Square F Ratio Prob $>$ F

$\begin{array}{llllll}\text { Blk/Sub-Blk } & 3 & 0.01696532 & 0.005655 & 11.1349 & 0.0032 *\end{array}$

$\begin{array}{llll}\text { Error } & 8 & 0.00406297 & 0.000508\end{array}$

$\begin{array}{lll}\text { C. Total } & 11 & 0.02102829\end{array}$

Means for Oneway Anova

Level Number Mean Std Error Lower 95\% Upper 95\%

$\begin{array}{llrrrr}1-1 & 3 & 1.48969 & 0.01301 & 1.4597 & 1.5197\end{array}$

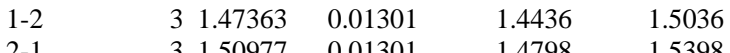

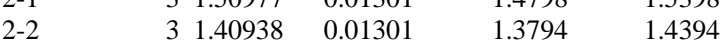

Std Error uses a pooled estimate of error variance 
Exhibit A-3. Analysis of Variance of Analytical Block Effects for Measurements of the LRM Standard Glass (continued)

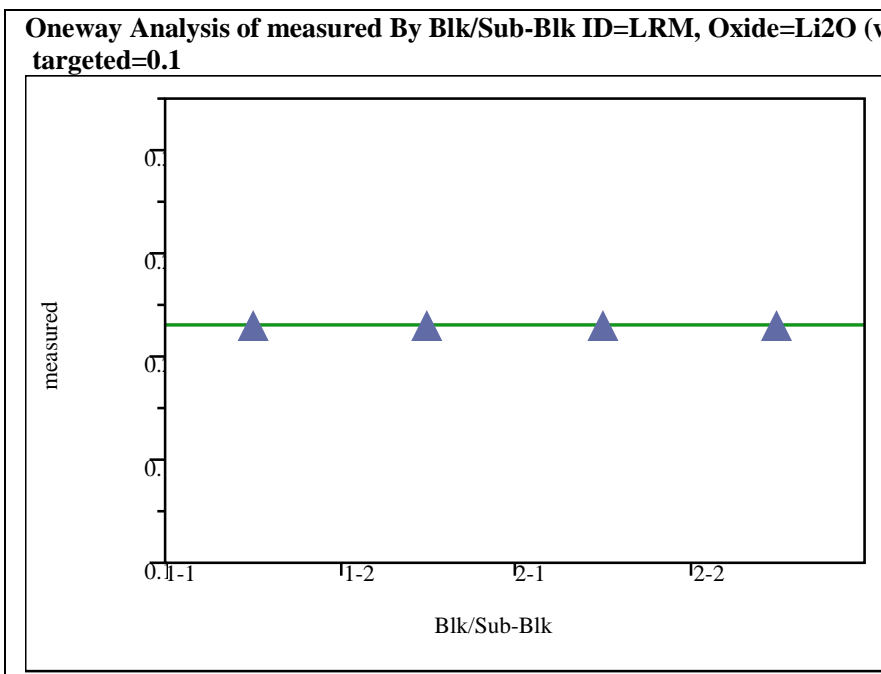

Oneway Anova

Summary of Fit

Rsquare

Adj Rsquare

Root Mean Square Error $\quad 0$

Mean of Response 0.21529

Observations (or Sum Wgts) 12

Analysis of Variance

Source DF Sum of Squares Mean Square F Ratio Prob $>$ F

Blk/Sub-Blk 3

$\begin{array}{lll}3 & 0 & 0 \\ 8 & 0 & 0\end{array}$

Error

11

0

Means for Oneway Anova

Level Number Mean Std Error Lower 95\% Upper 95\%

$\begin{array}{lllllr}1-1 & 3 & 0.215290 & 0 & 0.21529 & 0.21529 \\ 1-2 & 3 & 0.215290 & 0 & 0.21529 & 0.21529\end{array}$

$\begin{array}{llllll}2-1 & 3 & 0.215290 & 0 & 0.21529 & 0.21529\end{array}$

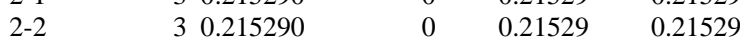

Std Error uses a pooled estimate of error variance

Oneway Analysis of measured By Blk/Sub-Blk ID=LRM, Oxide=MgO (wt\%), targeted $=0.1$

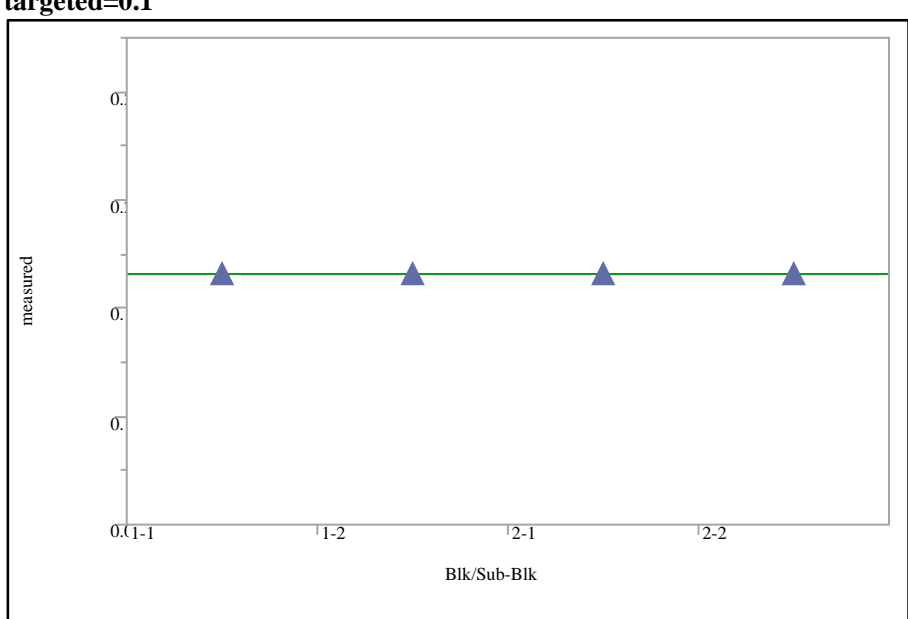

Oneway Anova

Summary of Fit

Rsquare

Adj Rsquare

Root Mean Square Error

Mean of Response

Observations (or Sum Wgts)

16583

Analysis of Variance

Source DF Sum of Squares Mean Square F Ratio Prob > F

Blk/Sub-Blk 3

Error

C. Total

8
11

0
0

Means for Oneway Anova

Level Number Mean Std Error Lower 95\% Upper 95\%

$\begin{array}{llllll}1-1 & 3 & 0.165830 & 0 & 0.16583 & 0.16583\end{array}$

$\begin{array}{llllll}1-2 & 3 & 0.165830 & 0 & 0.16583 & 0.16583\end{array}$

$\begin{array}{llllll}1-2 & 3 & 0.165830 & 0 & 0.16583 & 0.16583 \\ 2-1 & 3 & 0.165830 & 0 & 0.16583 & 0.16583\end{array}$

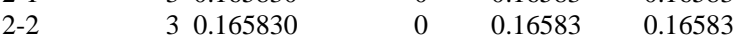

Std Error uses a pooled estimate of error variance 
Exhibit A-3. Analysis of Variance of Analytical Block Effects for Measurements of the LRM Standard Glass (continued)

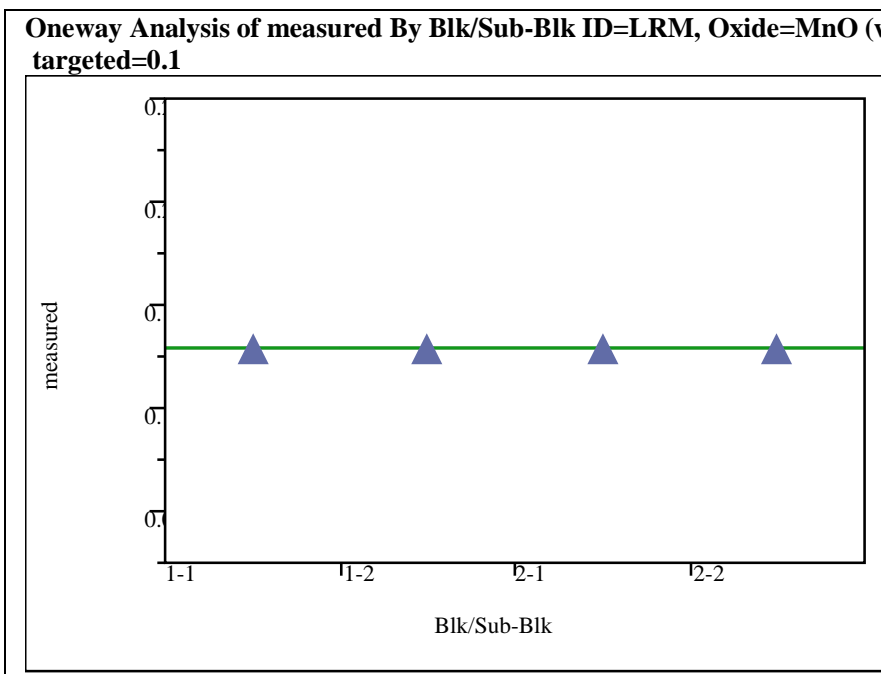

Oneway Anova

Summary of Fit

Rsquare

Adj Rsquare

Root Mean Square Error $\quad 0$

Mean of Response 0.12912

Observations (or Sum Wgts) 12

Analysis of Variance

Source DF Sum of Squares Mean Square F Ratio Prob > F

Blk/Sub-Blk 3

0

Error

C. Total

11

0

Oneway Analysis of measured By Blk/Sub-Blk ID=LRM, Oxide=Na2O (wt\%), targeted $=20$

Means for Oneway Anova

Level Number Mean Std Error Lower 95\% Upper 95\%

$\begin{array}{lrrrrr}\text { Lever } & \text { Number } & \text { Mean } & \text { Std Error } & \text { Lower 95\% } & \text { Upper 95\% } \\ 1-1 & 3 & 0.129120 & 0 & 0.12912 & 0.12912 \\ 1-2 & 3 & 0.129120 & 0 & 0.12912 & 0.12912 \\ 2-1 & 3 & 0.129120 & 0 & 0.12912 & 0.12912 \\ 2-2 & 3 & 0.129120 & 0 & 0.12912 & 0.12912\end{array}$

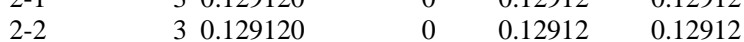

Std Error uses a pooled estimate of error variance

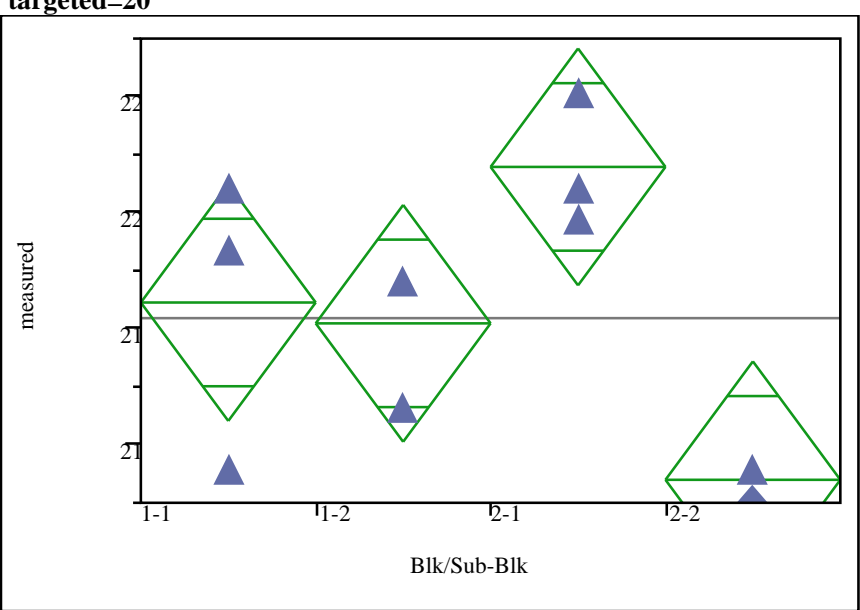

Oneway Anova

Summary of Fit

$\begin{array}{lr}\text { Rsquare } & 0.700155 \\ \text { Adj Rsquare } & 0.587713 \\ \text { Root Mean Square Error } & 0.383253 \\ \text { Mean of Response } & 21.54553 \\ \text { Observations (or Sum Wgts) } & 12\end{array}$

Analysis of Variance

Source DF Sum of Squares Mean Square F Ratio Prob $>$ F

$\begin{array}{llllll}\text { Blk/Sub-Blk } & 3 & 2.7438270 & 0.914609 & 6.2268 & 0.0173 *\end{array}$

$\begin{array}{llll}\text { Error } & 8 & 1.1750606 & 0.146883\end{array}$

C. Total $\quad 11 \quad 3.9188876$

Means for Oneway Anova

Level Number Mean Std Error Lower 95\% Upper 95\%

$\begin{array}{llllrr}1-1 & 3 & 21.6129 & 0.22127 & 21.103 & 22.123\end{array}$

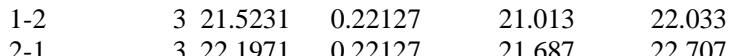

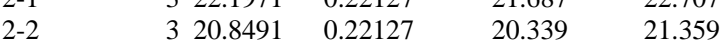

Std Error uses a pooled estimate of error variance 
Exhibit A-3. Analysis of Variance of Analytical Block Effects for Measurements of the LRM Standard Glass (continued)

Oneway Analysis of measured By Blk/Sub-Blk ID=LRM, Oxide=NiO (wt\%), targeted $=0.1$

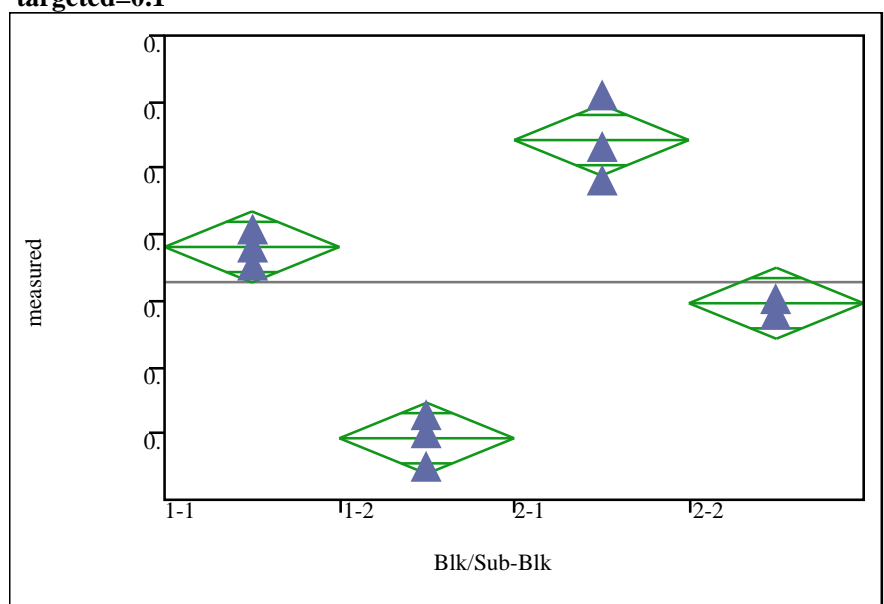

Oneway Anova

Summary of Fit

$\begin{array}{lr}\text { Rsquare } & 0.960546 \\ \text { Adj Rsquare } & 0.94575 \\ \text { Root Mean Square Error } & 0.002012 \\ \text { Mean of Response } & 0.156411 \\ \text { Observations (or Sum Wgts) } & 12\end{array}$

Analysis of Variance

Source DF Sum of Squares Mean Square F Ratio Prob $>$ F

Blk/Sub-Blk $3 \quad 0.00078844 \quad 0.00026364 .9222<.0001^{*}$

$\begin{array}{llll}\text { Error } & 8 & 0.00003239 & 4.048 \mathrm{e}-6\end{array}$

C. Total $\quad 11 \quad 0.00082083$

Means for Oneway Anova

Level Number Mean Std Error Lower 95\% Upper 95\%

$\begin{array}{llllll}1-1 & 3 & 0.159063 & 0.00116 & 0.15638 & 0.16174\end{array}$

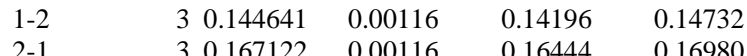

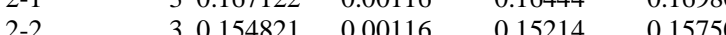

Std Error uses a pooled estimate of error variance
Oneway Analysis of measured By Blk/Sub-Blk ID=LRM, Oxide=P2O5 (wt\%), targeted $=0.5$

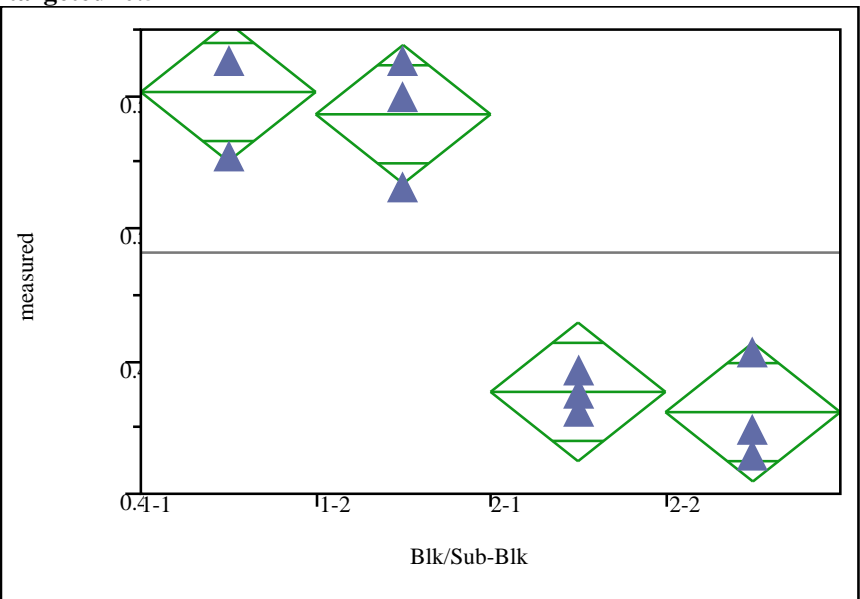

Oneway Anova

Summary of Fit

$\begin{array}{lr}\text { Rsquare } & 0.92543 \\ \text { Adj Rsquare } & 0.897466 \\ \text { Root Mean Square Error } & 0.019634 \\ \text { Mean of Response } & 0.490932 \\ \text { Observations (or Sum Wgts) } & 12\end{array}$

Analysis of Variance

Source DF Sum of Squares Mean Square F Ratio Prob $>$ F

$\begin{array}{llllll}\text { Blk/Sub-Blk } & 3 & 0.03827056 & 0.01275733 .0938 & <.0001 *\end{array}$

$\begin{array}{llll}\text { Error } & 8 & 0.00308380 & 0.000385\end{array}$

C. Total $\quad 11 \quad 0.04135436$

Means for Oneway Anova

Level Number Mean Std Error Lower 95\% Upper 95\%

$\begin{array}{lllllr}1-1 & 3 & 0.551464 & 0.01134 & 0.52532 & 0.57760\end{array}$

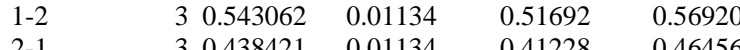

30.430783

Std Error uses a pooled estimate of error variance 
Exhibit A-3. Analysis of Variance of Analytical Block Effects for Measurements of the LRM Standard Glass (continued)

Oneway Analysis of measured By Blk/Sub-Blk ID=LRM, Oxide=PbO (wt\%), targeted $=0.1$

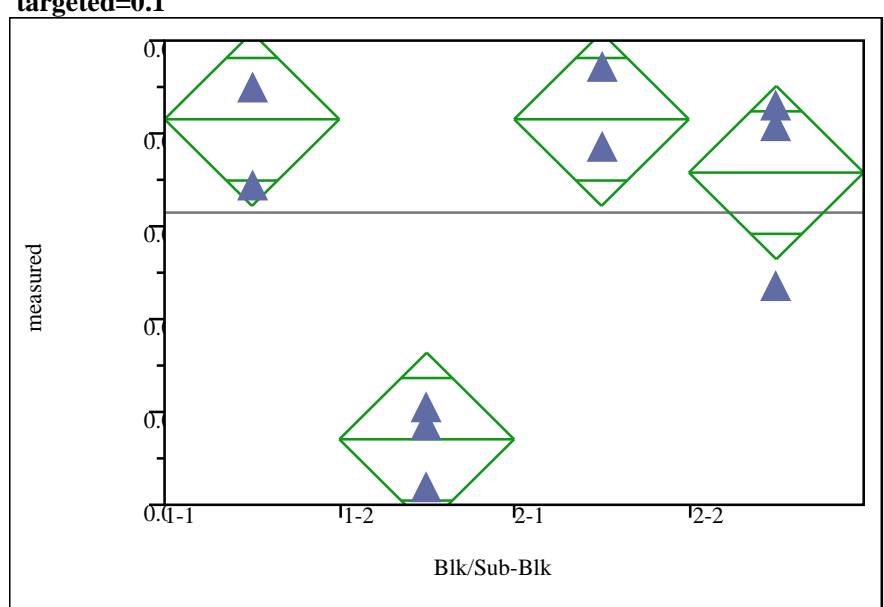

Oneway Anova

Summary of Fit

$\begin{array}{lr}\text { Rsquare } & 0.861806 \\ \text { Adj Rsquare } & 0.809984 \\ \text { Root Mean Square Error } & 0.003504 \\ \text { Mean of Response } & 0.070736 \\ \text { Observations (or Sum Wgts) } & 12\end{array}$

Analysis of Variance

Source DF Sum of Squares Mean Square F Ratio Prob > F

Blk/Sub-Blk $3 \quad 0.00061267 \quad 0.00020416 .6299 \quad 0.0008^{*}$

$\begin{array}{llll}\text { Error } & 8 & 0.00009824 & 0.000012\end{array}$

C. Total $\quad 11 \quad 0.00071091$

Means for Oneway Anova

Level Number Mean Std Error Lower 95\% Upper 95\%

$\begin{array}{llllll}1-1 & 3 & 0.075763 & 0.00202 & 0.07110 & 0.08043\end{array}$

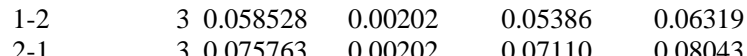

$2-2 \quad 30.072891-0.00202-0.06822-0.07756$

Std Error uses a pooled estimate of error variance
Oneway Analysis of measured By Blk/Sub-Blk ID=LRM, Oxide=RuO2 (wt\%), targeted $=\mathbf{0}$

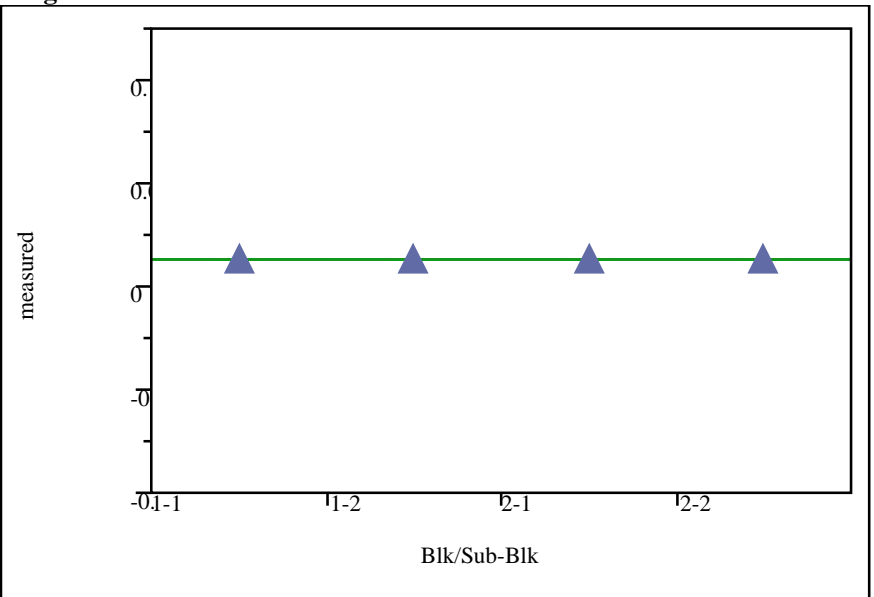

Oneway Anova

Summary of Fit

Rsquare

Adj Rsquare

0
-0.375

Root Mean Square Error $\quad 2.12 \mathrm{e}-18$
$\quad$

$\begin{array}{lr}\text { Mean of Response } & 0.013166 \\ \text { Observations (or Sum Wgts) } & 12\end{array}$

Analysis of Variance

Source DF Sum of Squares Mean Square F Ratio Prob $>$ F

$\begin{array}{lrrrrr}\text { Blk/Sub-Blk } & 3 & 0 & 0 & 0.0000 & 1.0000\end{array}$

$\begin{array}{llll}\text { Error } & 8 & 3.6111 \mathrm{e}-35 & 4.514 \mathrm{e}-36\end{array}$

C. Total $11 \quad 3.6111 \mathrm{e}-35$

Means for Oneway Anova

Level Number Mean Std Error Lower 95\% Upper 95\%

$\begin{array}{lllllr}1-1 & 3 & 0.013166 & 1.227 \mathrm{e}-18 & 0.01317 & 0.01317\end{array}$

$\begin{array}{llllll}1-2 & 3 & 0.013166 & 1.227 \mathrm{e}-18 & 0.01317 & 0.01317\end{array}$

$\begin{array}{llllll}2-1 & 3 & 0.013166 & 1.227 \mathrm{e}-18 & 0.01317 & 0.01317\end{array}$

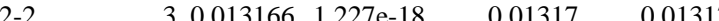

Std Error uses a pooled estimate of error variance 
Exhibit A-3. Analysis of Variance of Analytical Block Effects for Measurements of the LRM Standard Glass (continued)

Oneway Analysis of measured By Blk/Sub-Blk ID=LRM, Oxide=SiO2 (wt\%), targeted $=54$

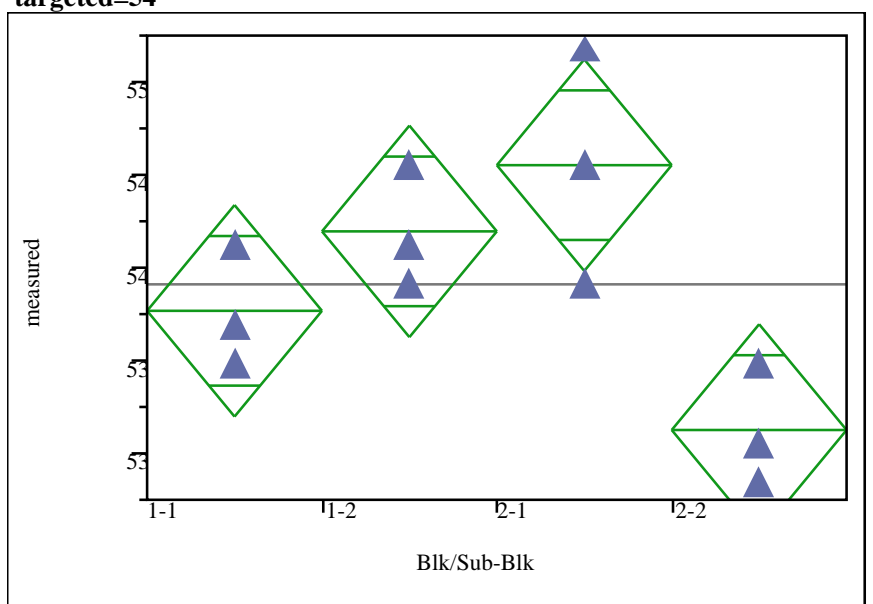

Oneway Anova

Summary of Fit

$$
\begin{array}{lr}
\text { Rsquare } & 0.698113 \\
\text { Adj Rsquare } & 0.584906 \\
\text { Root Mean Square Error } & 0.42786 \\
\text { Mean of Response } & 53.91036 \\
\text { Observations (or Sum Wgts) } & 12
\end{array}
$$

\section{Analysis of Variance}

Source DF Sum of Squares Mean Square F Ratio Prob $>$ F

Blk/Sub-Blk $3 \quad 3.3866873 \quad 1.12890 \quad 6.1667 \quad 0.0178^{*}$

Error $\quad 8 \quad 1.4645134$

\begin{tabular}{|c|c|c|c|c|}
\hline $1-1$ & 353.7677 & 0.24703 & 53.198 & 54.337 \\
\hline $1-2$ & 354.1956 & 0.24703 & 53.626 & 54.765 \\
\hline $2-1$ & 354.5522 & 0.24703 & 53.983 & 55.122 \\
\hline $2-2$ & 353.1260 & 0.24703 & 52.556 & 53.696 \\
\hline
\end{tabular}

0.18306

C. Total $\quad 11 \quad 4.8512008$

Means for Oneway Anova

Level Number Mean Std Error Lower 95\% Upper 95\%

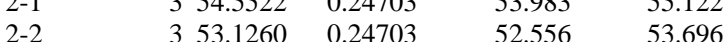

Std Error uses a pooled estimate of error variance
Oneway Analysis of measured By Blk/Sub-Blk ID=LRM, Oxide=SO3 (wt\%), targeted $=0.2$

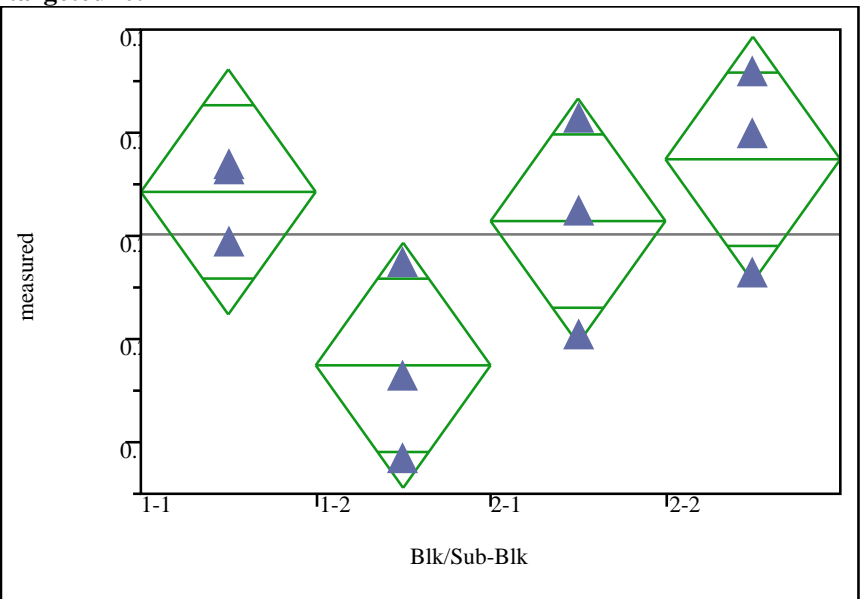

Oneway Anova

Summary of Fit

$\begin{array}{lr}\text { Rsquare } & 0.522931 \\ \text { Adj Rsquare } & 0.34403 \\ \text { Root Mean Square Error } & 0.044619 \\ \text { Mean of Response } & 0.25073 \\ \text { Observations (or Sum Wgts) } & 12\end{array}$

Analysis of Variance

Source DF Sum of Squares Mean Square F Ratio Prob $>$ F

$\begin{array}{llllll}\text { Blk/Sub-Blk } & 3 & 0.01745819 & 0.005819 & 2.9230 & 0.1001\end{array}$

$\begin{array}{llll}\text { Error } & 8 & 0.01592709 & 0.001991\end{array}$

C. Total $\quad 11 \quad 0.03338528$

Means for Oneway Anova

Level Number Mean Std Error Lower 95\% Upper 95\%

$\begin{array}{llllll}1-1 & 3 & 0.271330 & 0.02576 & 0.21192 & 0.33073\end{array}$

$\begin{array}{llllll}1-2 & 3 & 0.187268 & 0.02576 & 0.12786 & 0.24667\end{array}$

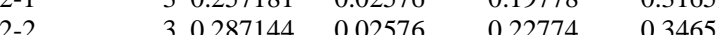

Std Error uses a pooled estimate of error variance 
Exhibit A-3. Analysis of Variance of Analytical Block Effects for Measurements of the LRM Standard Glass (continued)

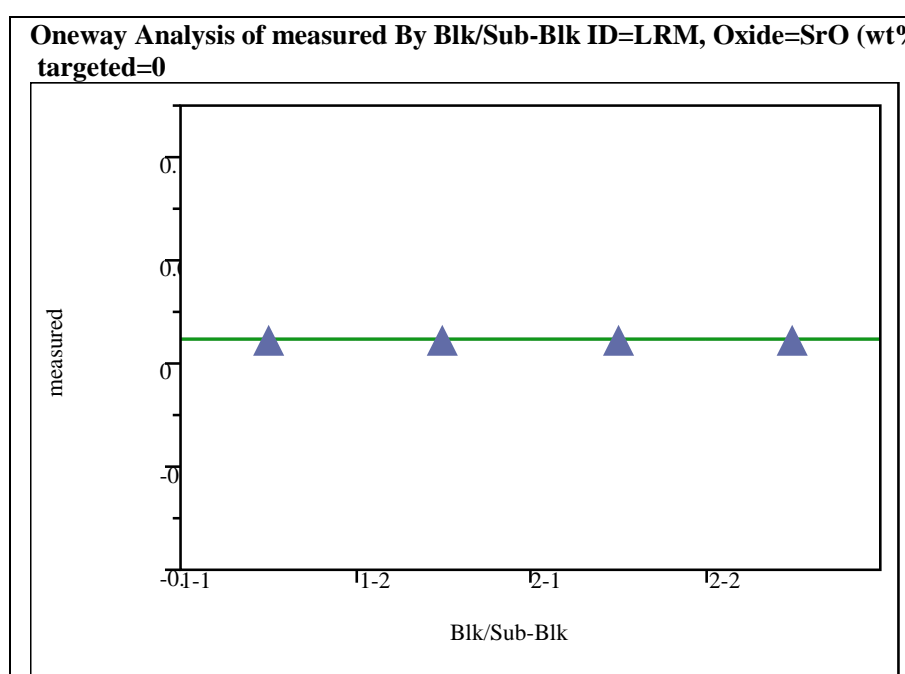

Oneway Anova

Summary of Fit

Rsquare

Adj Rsquare

Root Mean Square Error $\quad 0$

Mean of Response 0.011826

$\begin{array}{lr}\text { Observations (or Sum Wgts) } & 12\end{array}$

Analysis of Variance

Source DF Sum of Squares Mean Square F Ratio Prob $>$ F

Blk/Sub-Blk 3

0

Error

C. Total

11

Oneway Analysis of measured By Blk/Sub-Blk ID=LRM, Oxide=ZrO2 (wt\%), targeted $=1$

Means for Oneway Anova

Level Number Mean Std Error Lower 95\% Upper 95\%

$\begin{array}{lllllr}1-1 & 3 & 0.011826 & 0 & 0.01183 & 0.01183\end{array}$

$\begin{array}{llllll}1-2 & 3 & 0.011826 & 0 & 0.01183 & 0.01183 \\ 2-1 & 3 & 0.011826 & 0 & 0.01183 & 0.01183\end{array}$

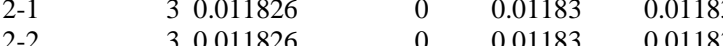

Std Error uses a pooled estimate of error variance

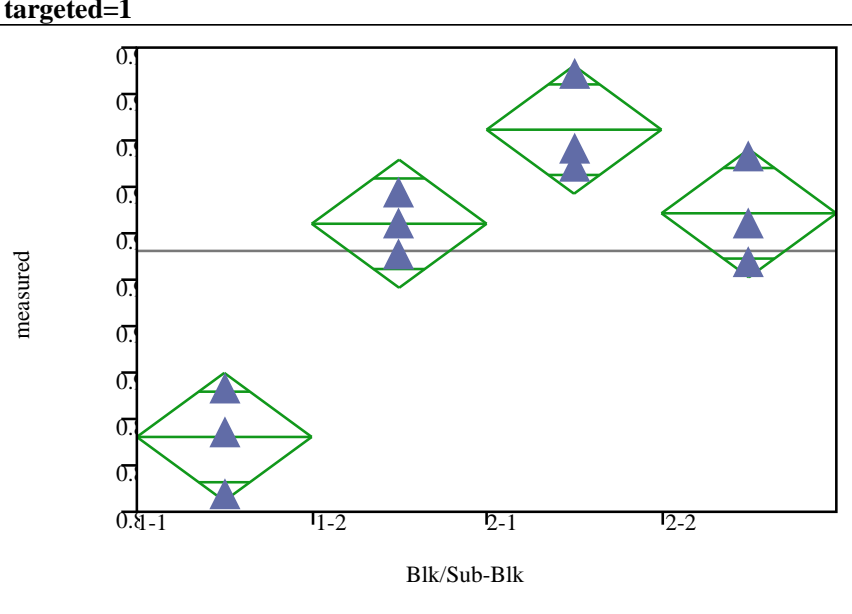

Oneway Anova

Summary of Fit

$\begin{array}{lr}\text { Rsquare } & 0.892949 \\ \text { Adj Rsquare } & 0.852805 \\ \text { Root Mean Square Error } & 0.010361 \\ \text { Mean of Response } & 0.926199 \\ \text { Observations (or Sum Wgts) } & 12\end{array}$

Analysis of Variance

Source DF Sum of Squares Mean Square F Ratio Prob $>$ F

$\begin{array}{llllll}\text { Blk/Sub-Blk } & 3 & 0.00716362 & 0.00238822 .2436 & 0.0003 *\end{array}$

$\begin{array}{llll}\text { Error } & 8 & 0.00085881 & 0.000107\end{array}$

$\begin{array}{lll}\text { C. Total } & 11 & 0.00802242\end{array}$

Means for Oneway Anova

Level Number Mean Std Error Lower 95\% Upper 95\%

$\begin{array}{lllllr}1-1 & 3 & 0.886125 & 0.00598 & 0.87233 & 0.89992\end{array}$

$\begin{array}{llllll}1-2 & 3 & 0.932052 & 0.00598 & 0.91826 & 0.94585\end{array}$

$\begin{array}{llllll}2-1 & 3 & 0.952314 & 0.00598 & 0.93852 & 0.96611\end{array}$

$\begin{array}{lllll}2-2 & 3 & 0.934303 & 0.00598 & 0.92051\end{array}$

Std Error uses a pooled estimate of error variance 


\section{Exhibit A-4. Measured versus Targeted Concentrations by Glass ID by Oxide.}

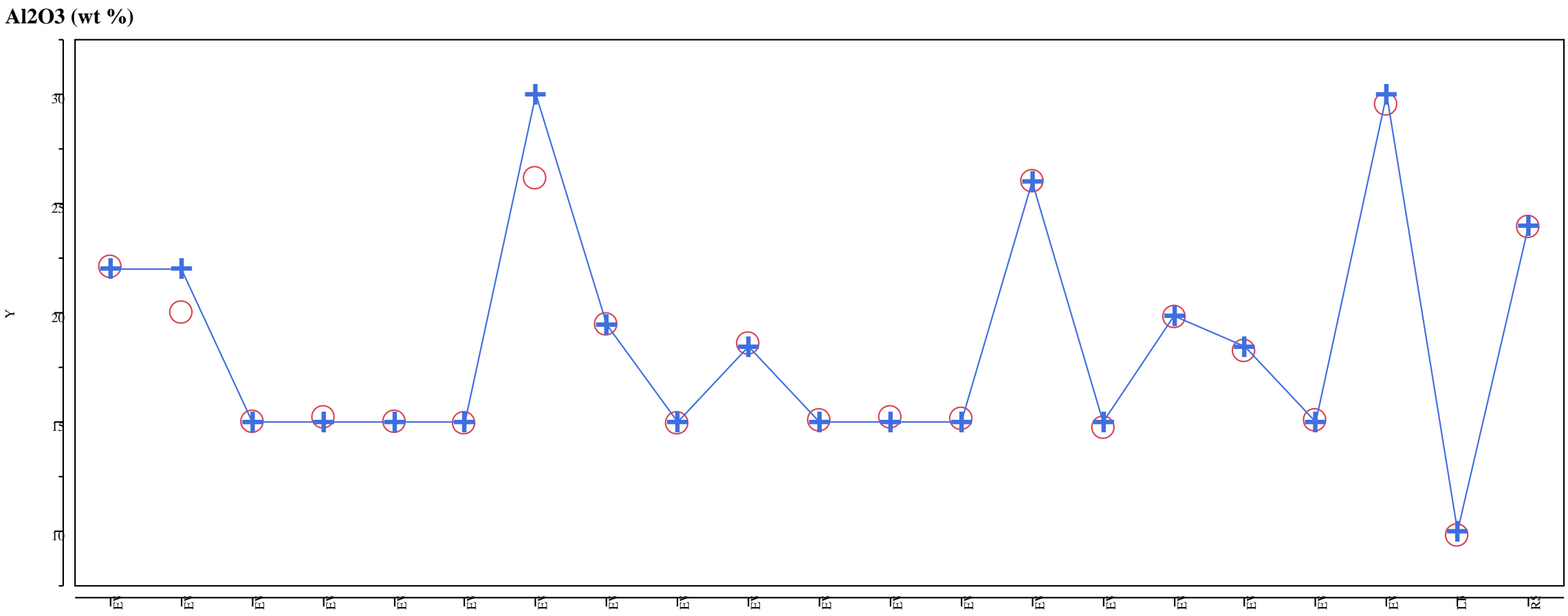

Y $\bigcirc$ Measured $\quad+-$ Targeted 
Exhibit A-4. Measured versus Targeted Concentrations by Glass ID by Oxide. (continued)

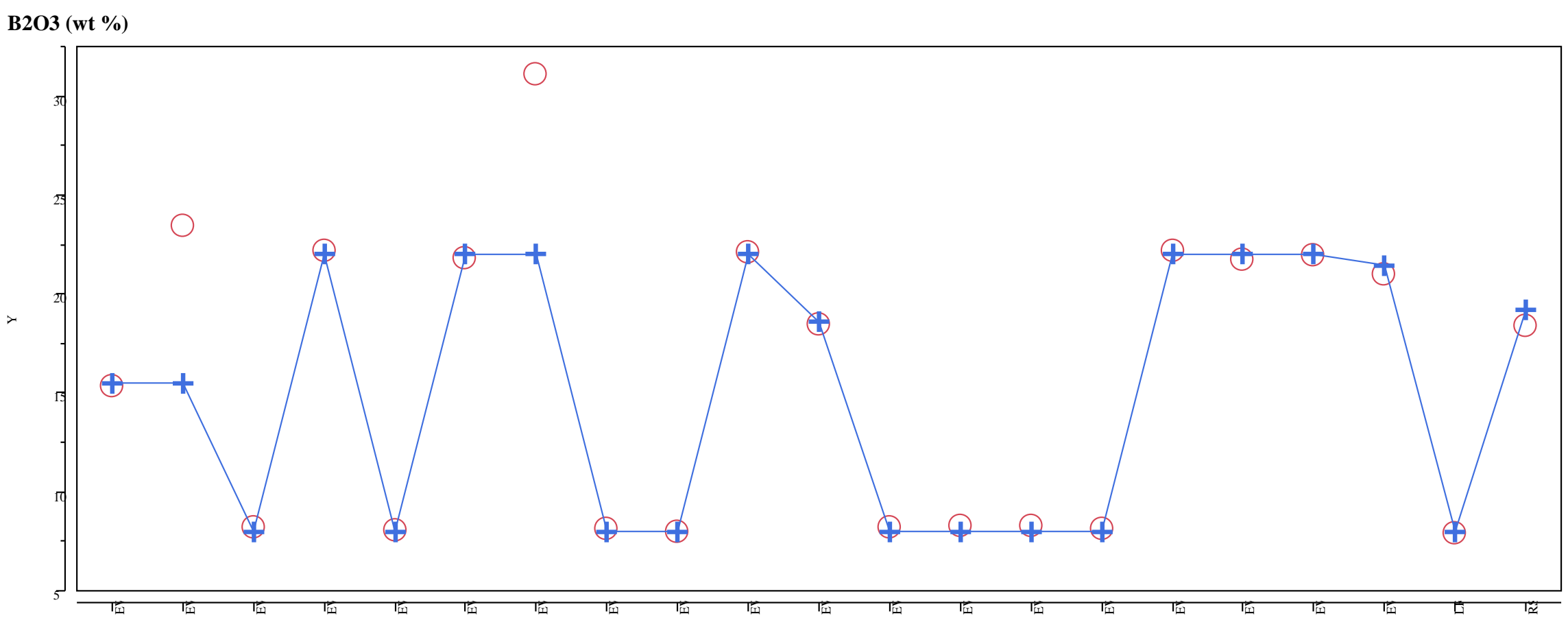


Exhibit A-4. Measured versus Targeted Concentrations by Glass ID by Oxide. (continued)

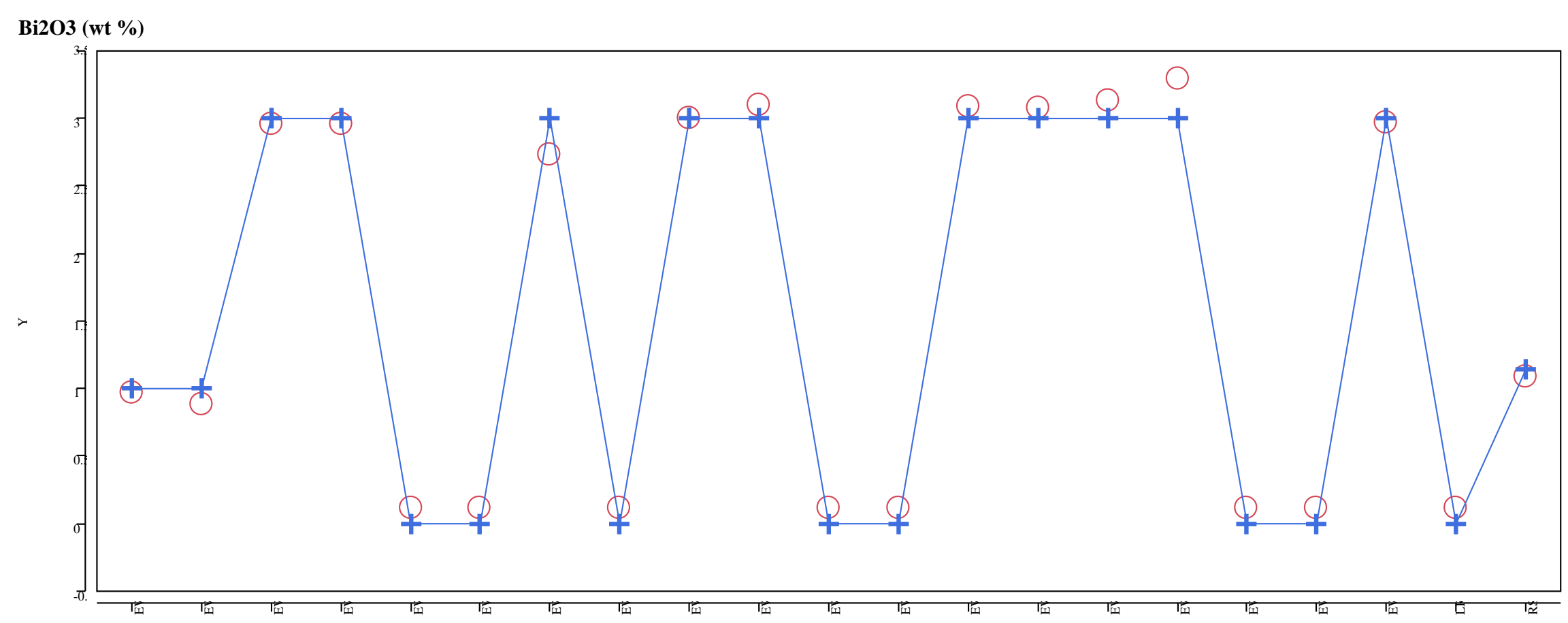

Y $\bigcirc$ Measured $\quad+-$ Targeted 
Exhibit A-4. Measured versus Targeted Concentrations by Glass ID by Oxide. (continued)

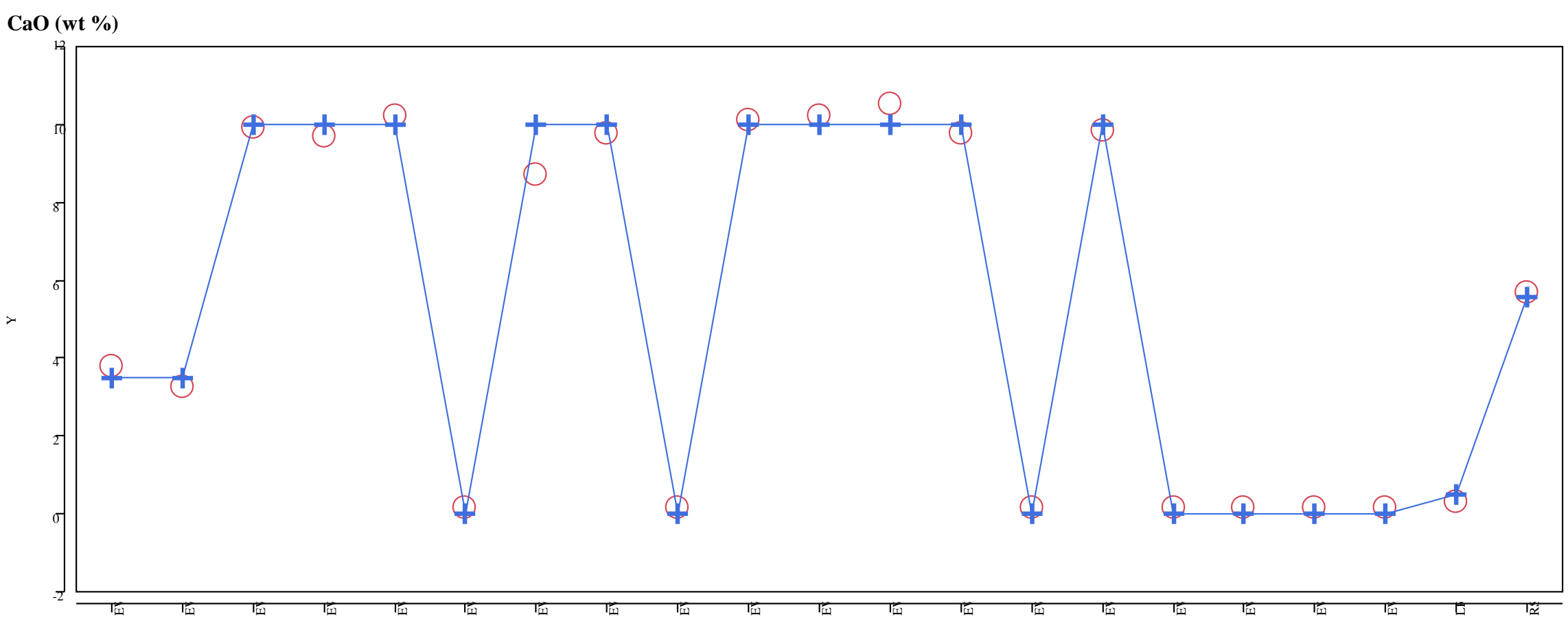


Exhibit A-4. Measured versus Targeted Concentrations by Glass ID by Oxide. (continued)

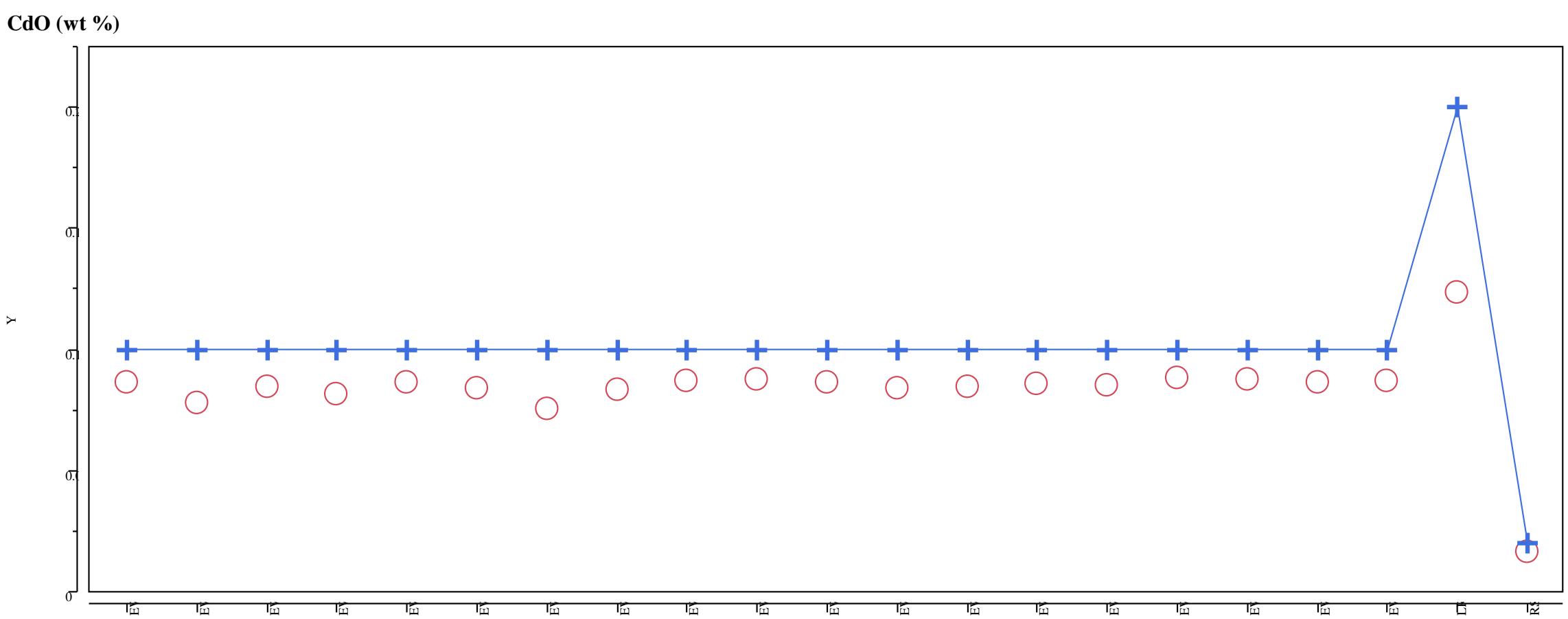


Exhibit A-4. Measured versus Targeted Concentrations by Glass ID by Oxide. (continued)

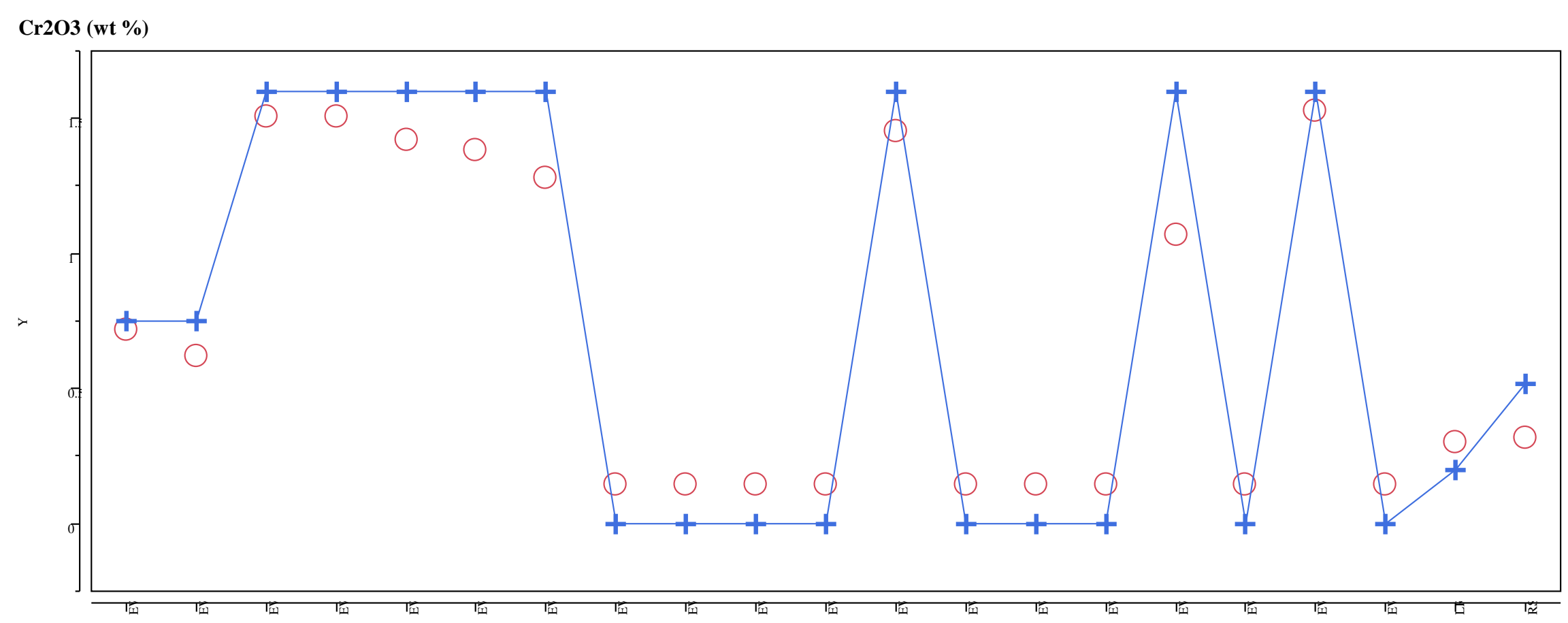


Exhibit A-4. Measured versus Targeted Concentrations by Glass ID by Oxide. (continued)

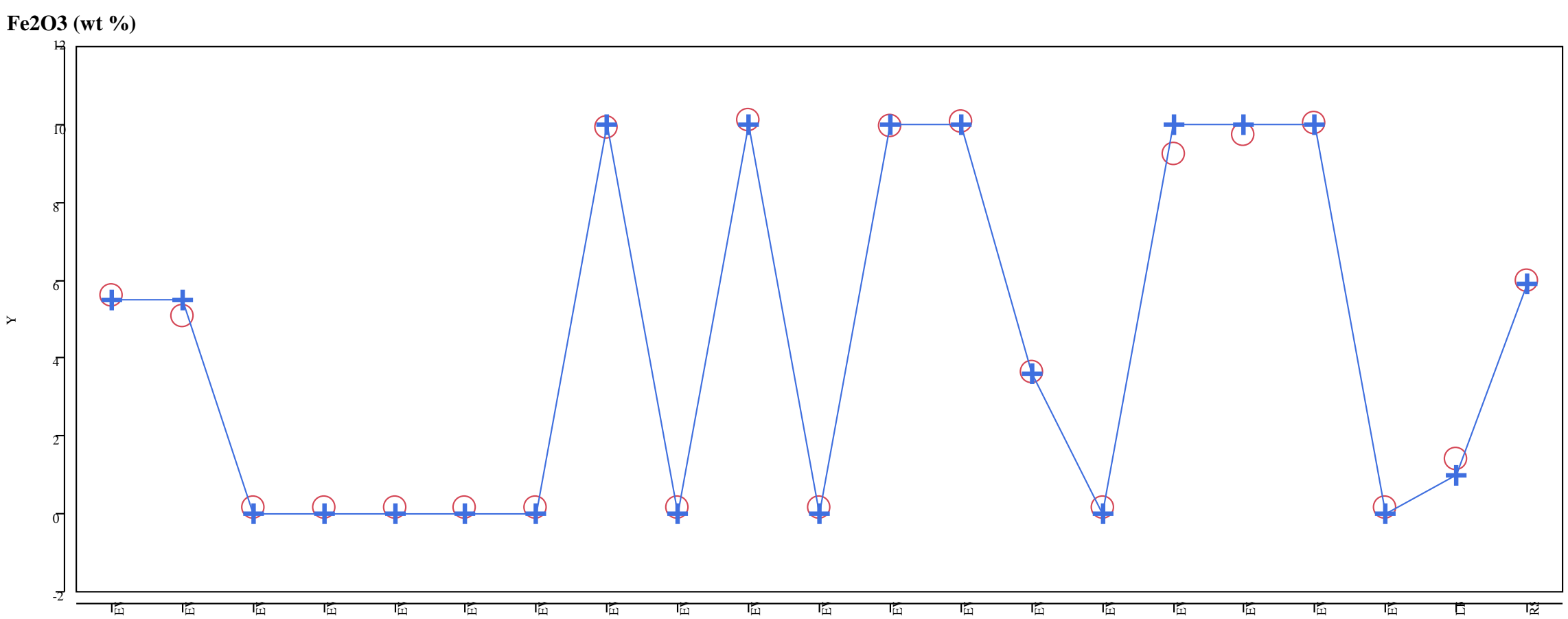


Exhibit A-4. Measured versus Targeted Concentrations by Glass ID by Oxide. (continued)

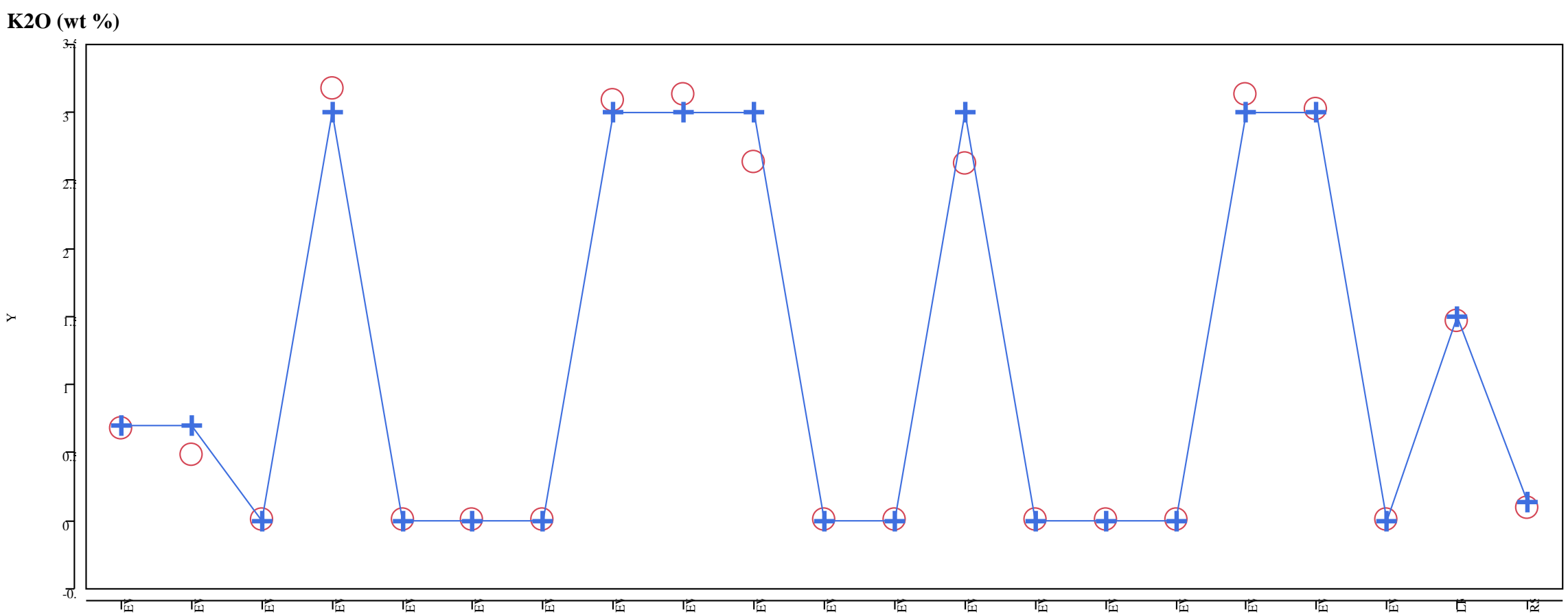

Y $\bigcirc$ Measured $\quad+-$ Targeted 
Exhibit A-4. Measured versus Targeted Concentrations by Glass ID by Oxide. (continued)

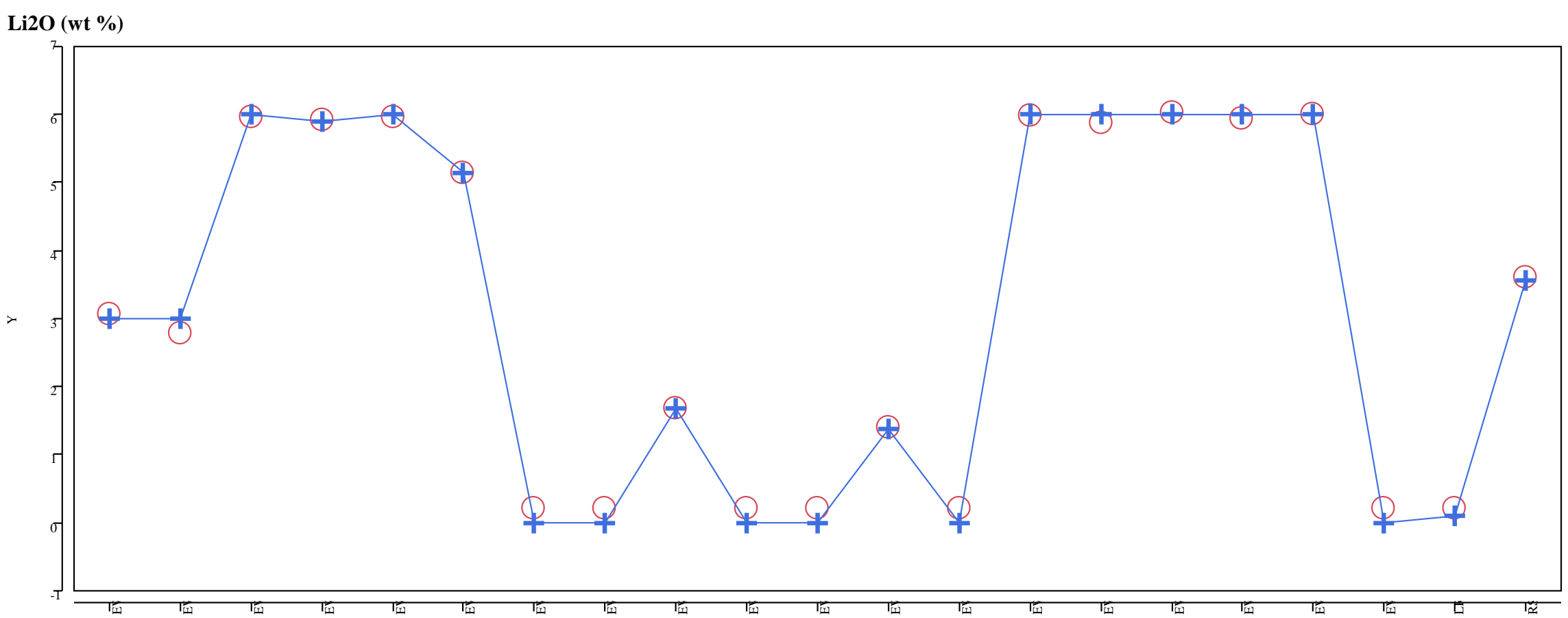


Exhibit A-4. Measured versus Targeted Concentrations by Glass ID by Oxide. (continued)

\section{MgO (wt \%)}

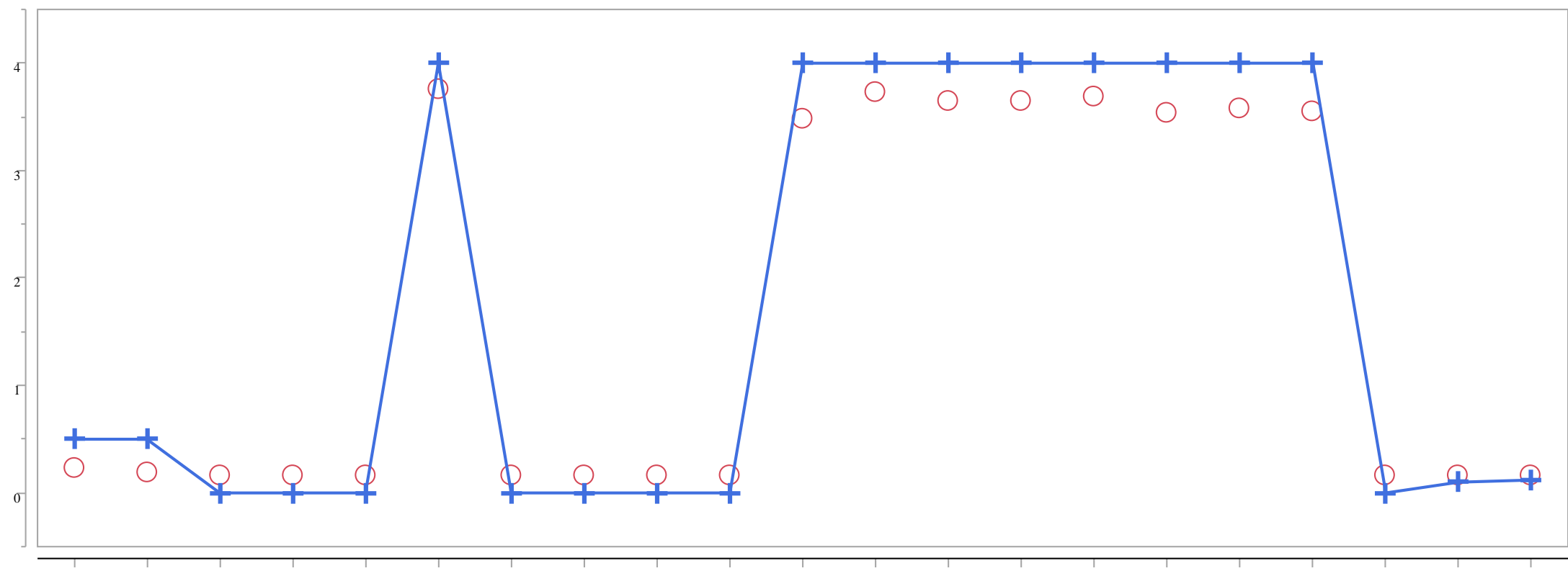

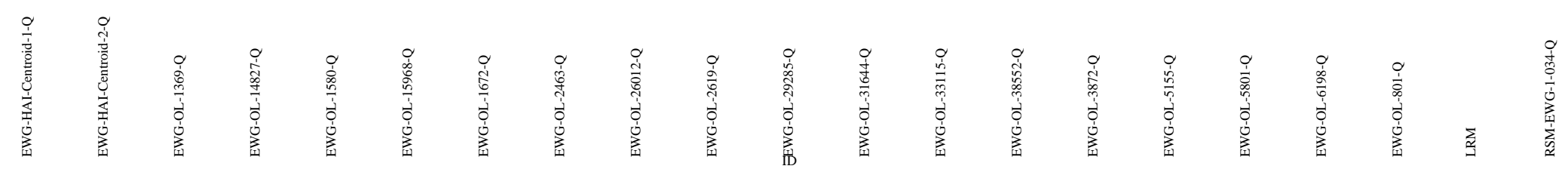

Y $\bigcirc$ Measured $\quad+$ Targeted $_{\text {T }}$ 
Exhibit A-4. Measured versus Targeted Concentrations by Glass ID by Oxide. (continued)

\section{MnO (wt \%)}

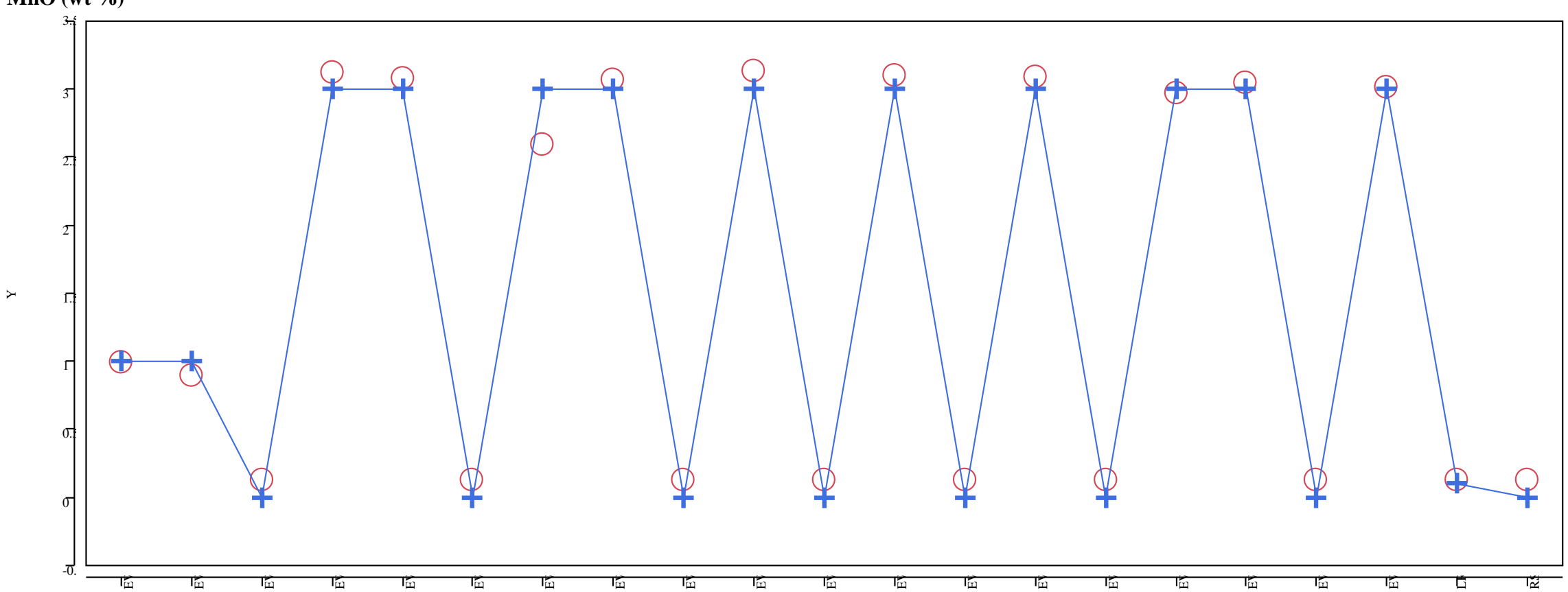

ID

Y $\bigcirc$ Measured $\quad+-$ Targeted 
Exhibit A-4. Measured versus Targeted Concentrations by Glass ID by Oxide. (continued)

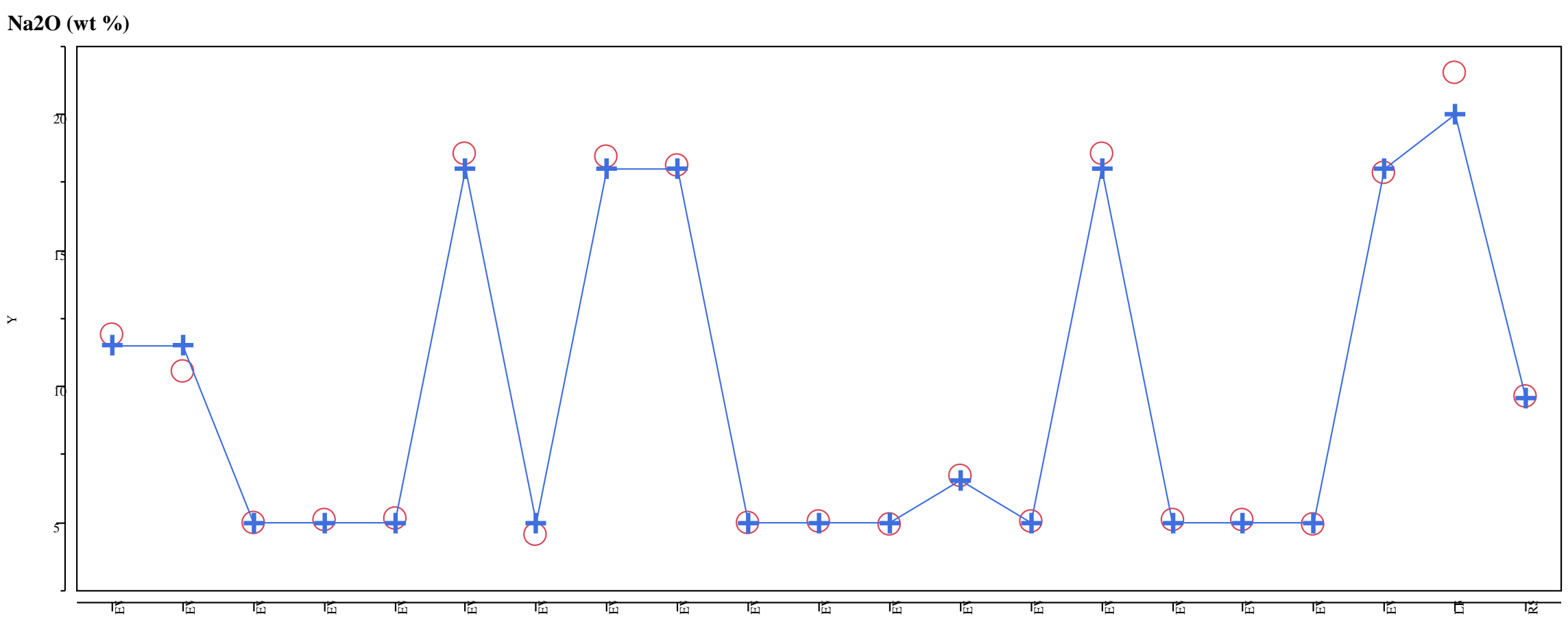


Exhibit A-4. Measured versus Targeted Concentrations by Glass ID by Oxide. (continued)

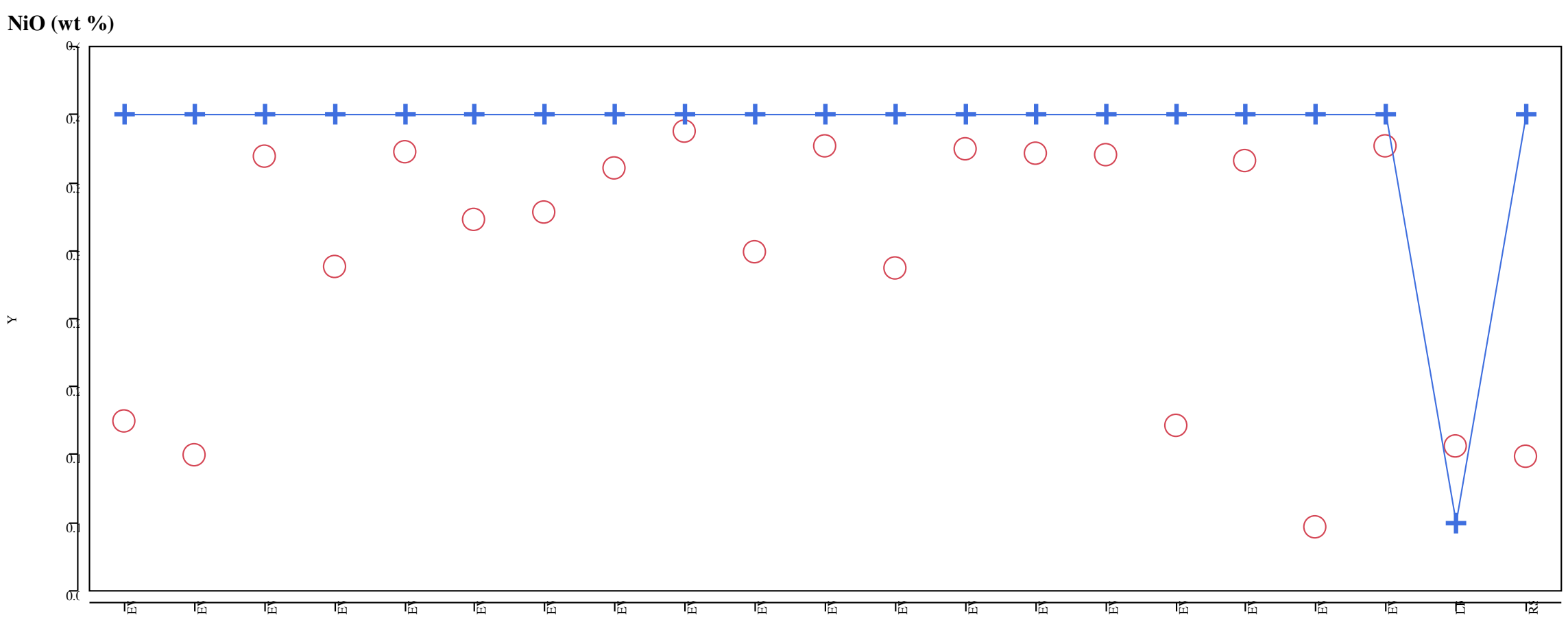


Exhibit A-4. Measured versus Targeted Concentrations by Glass ID by Oxide. (continued)

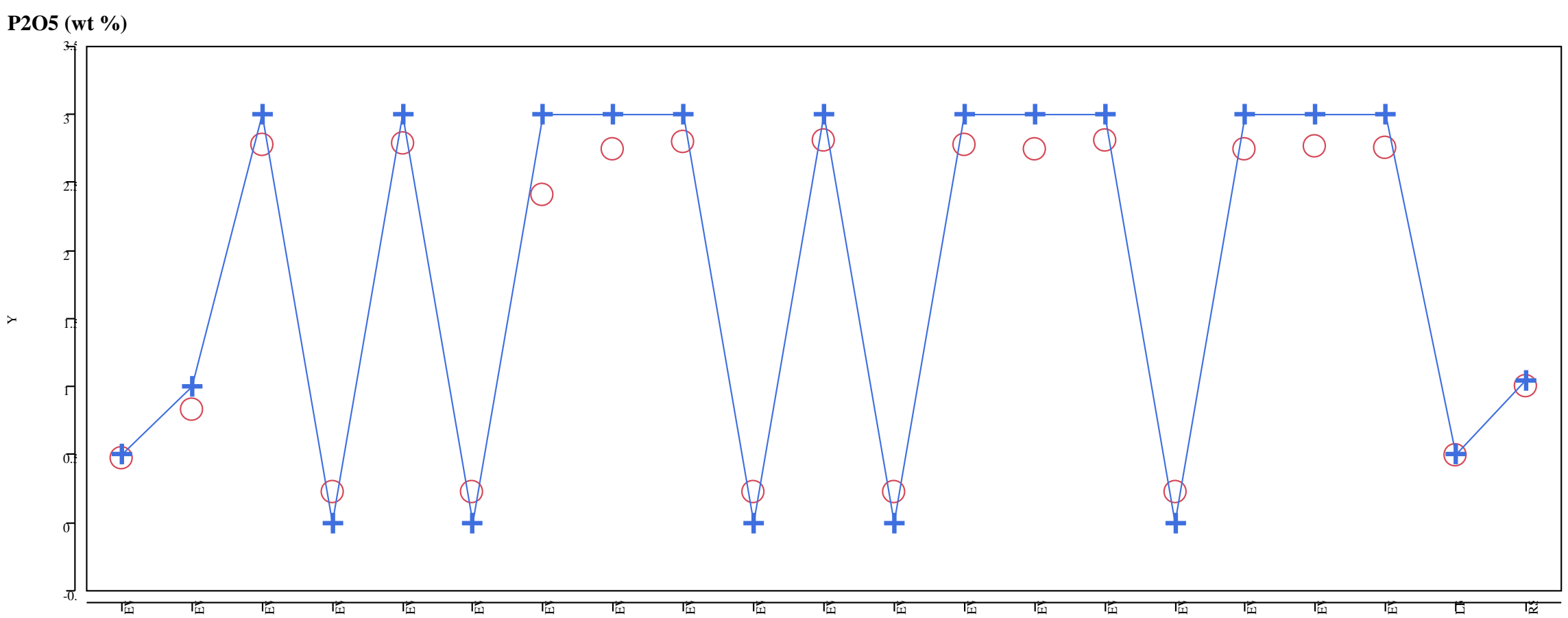


Exhibit A-4. Measured versus Targeted Concentrations by Glass ID by Oxide. (continued)

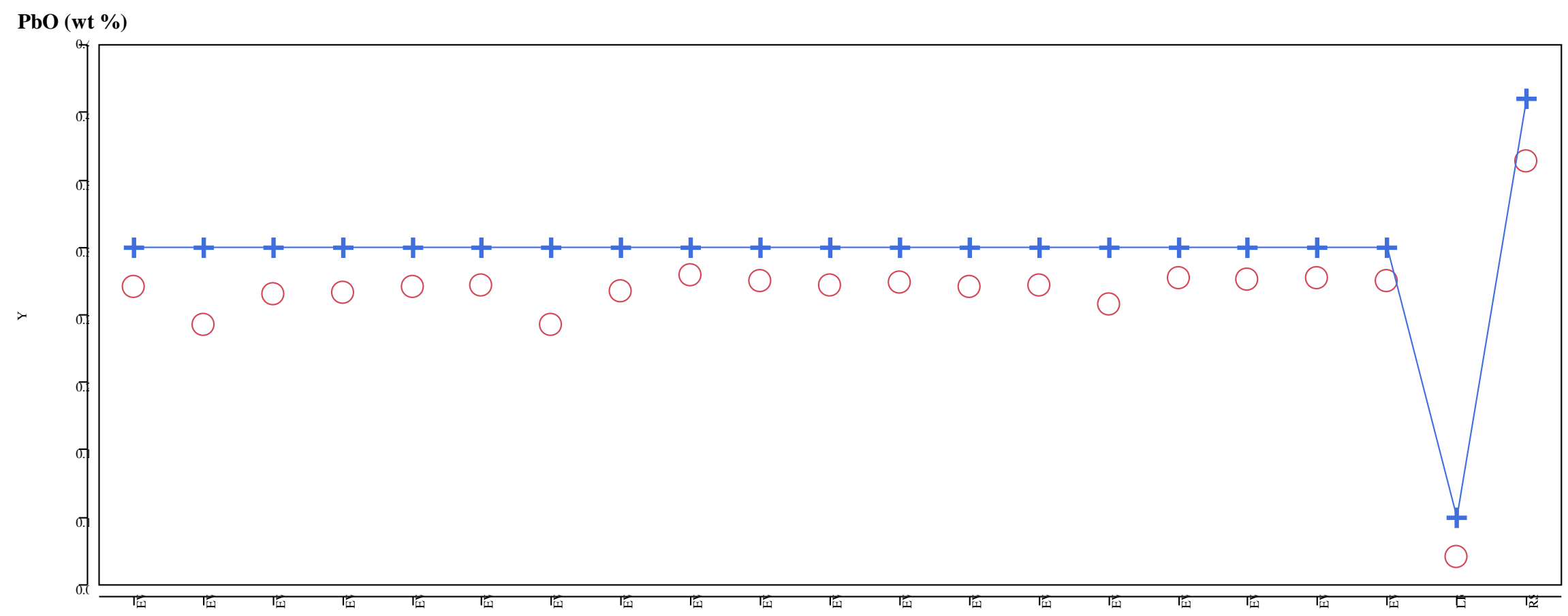


Exhibit A-4. Measured versus Targeted Concentrations by Glass ID by Oxide. (continued)

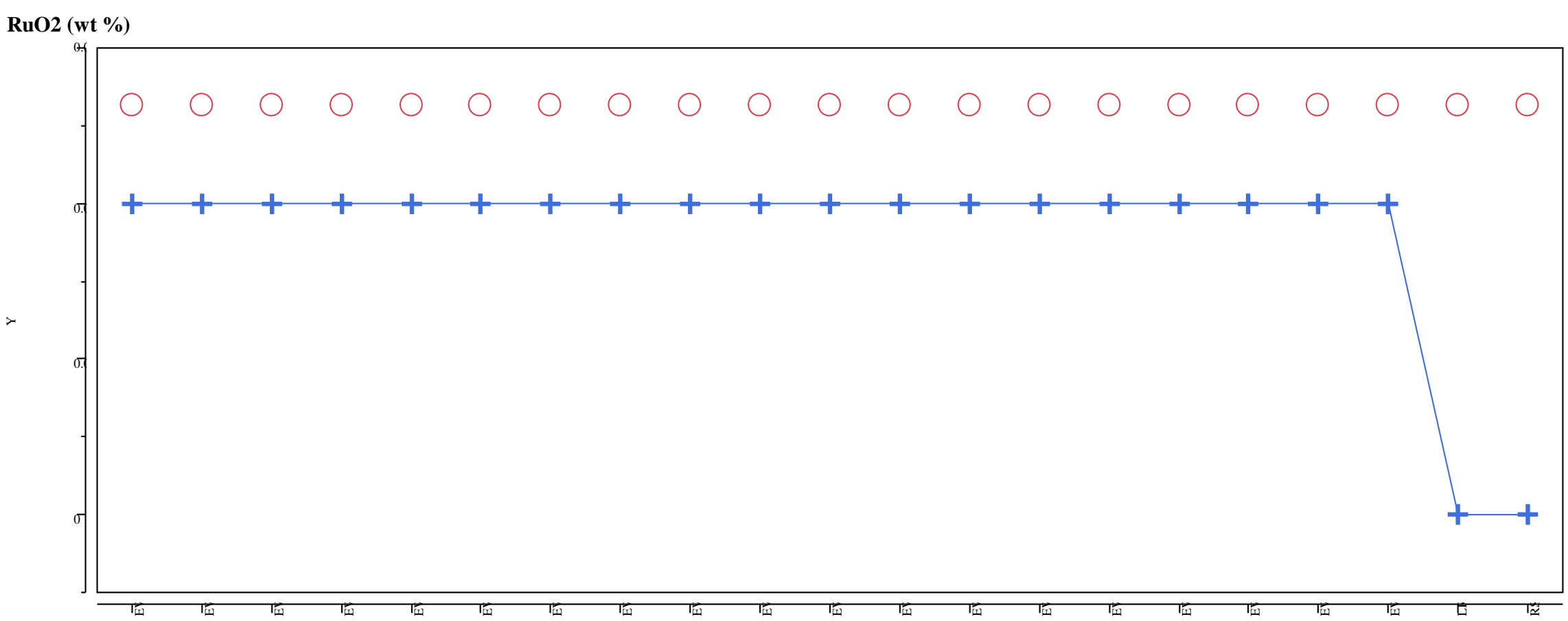


Exhibit A-4. Measured versus Targeted Concentrations by Glass ID by Oxide. (continued)

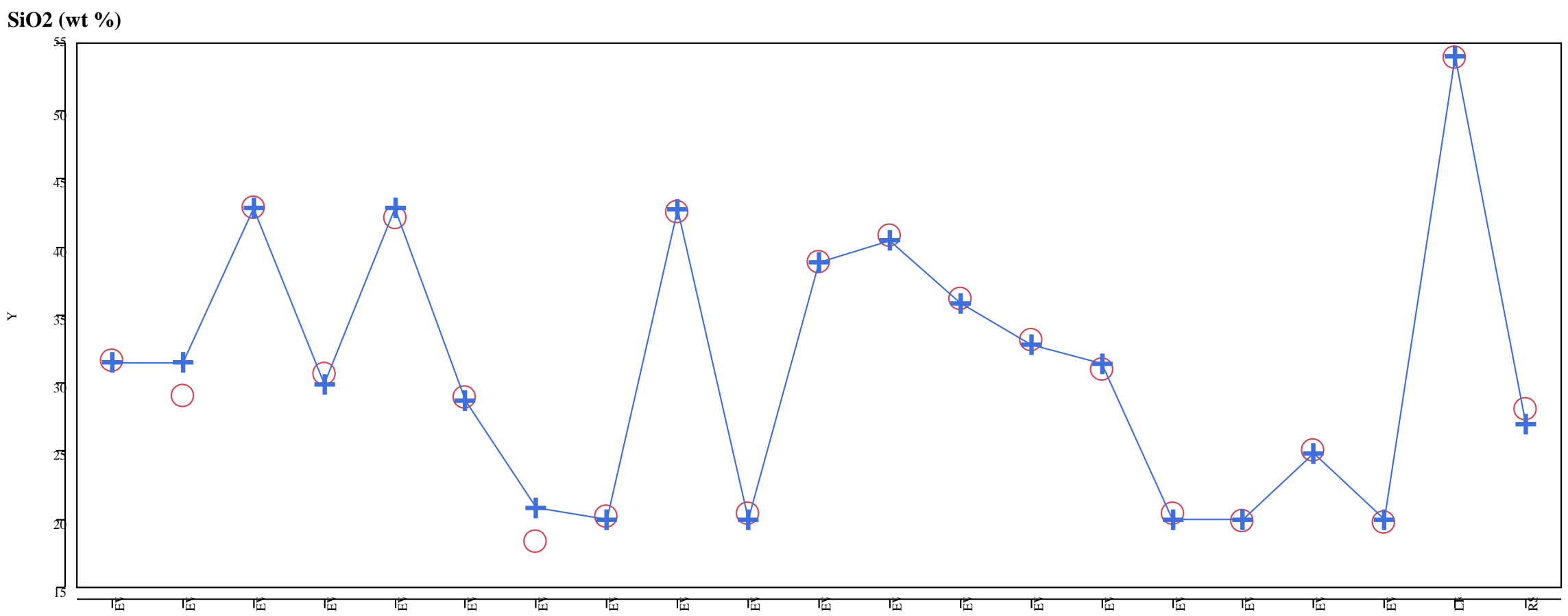


Exhibit A-4. Measured versus Targeted Concentrations by Glass ID by Oxide. (continued)

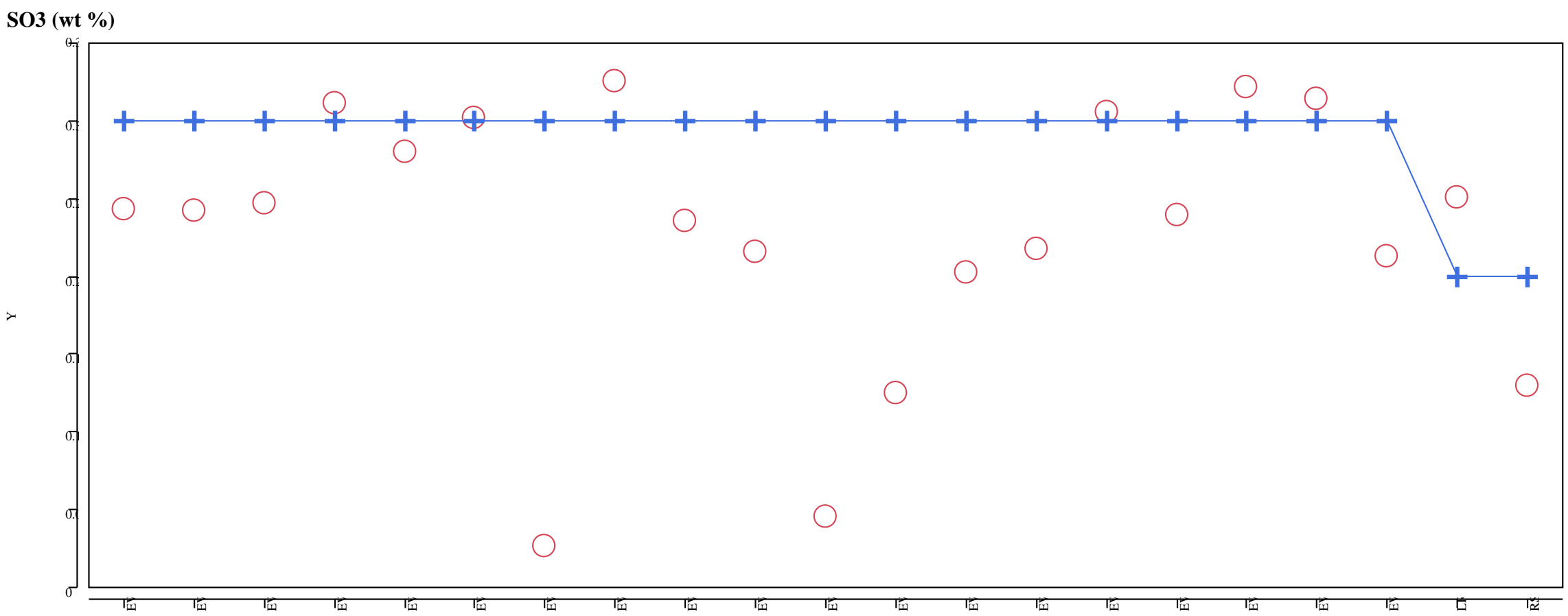


Exhibit A-4. Measured versus Targeted Concentrations by Glass ID by Oxide. (continued)

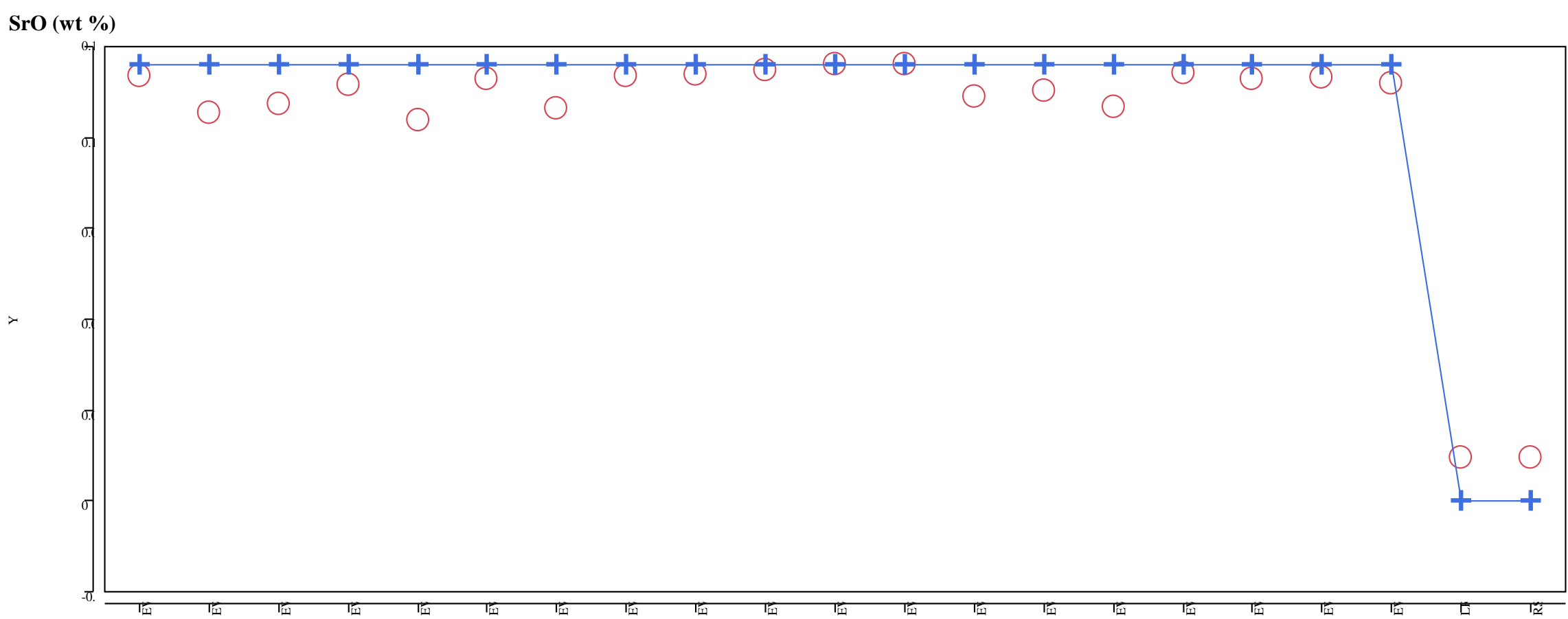


Exhibit A-4. Measured versus Targeted Concentrations by Glass ID by Oxide. (continued)

\section{$\mathrm{ZrO2}$ (wt \%)}

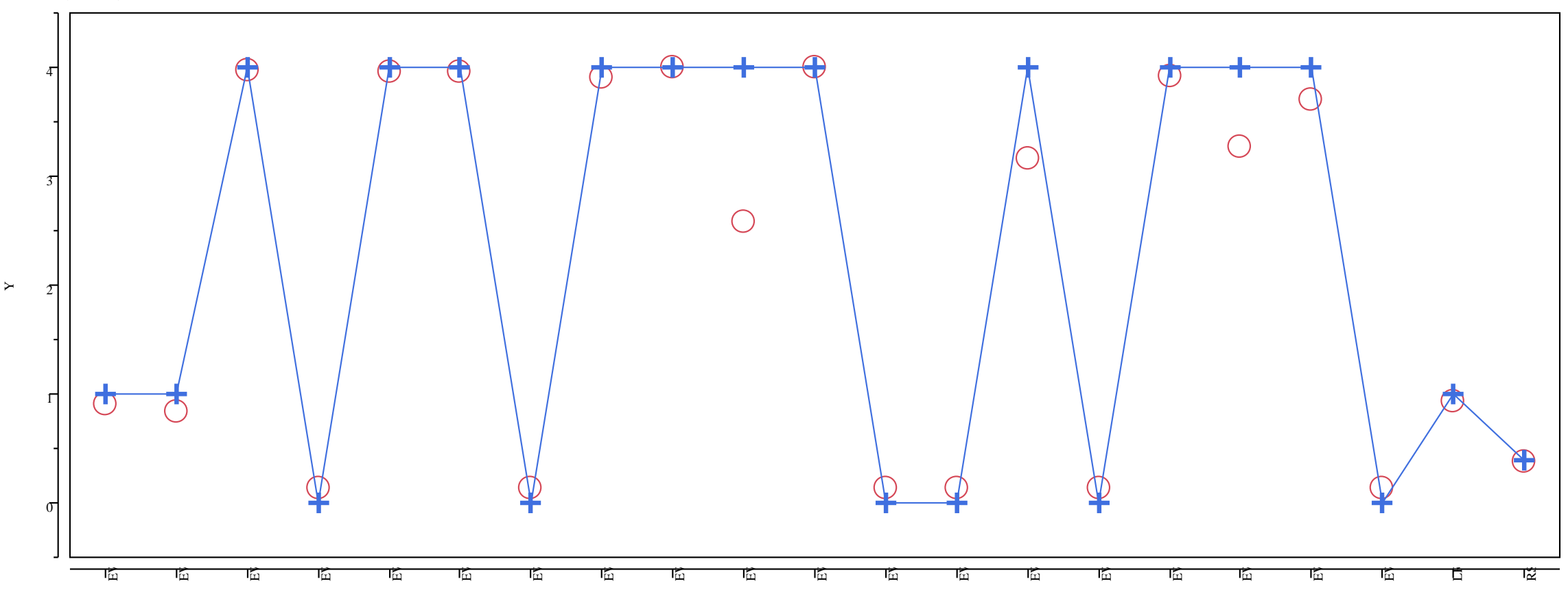


Exhibit A-4. Measured versus Targeted Concentrations by Glass ID by Oxide. (continued)

\section{Sum of Oxides}

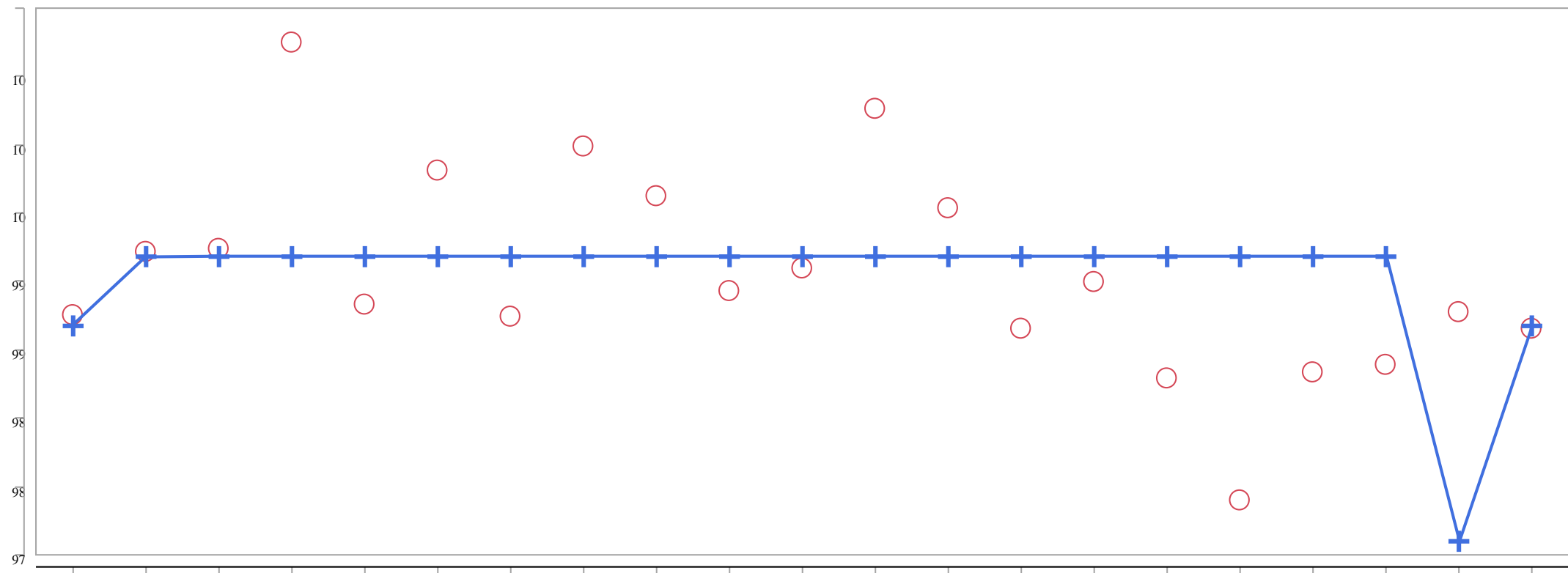

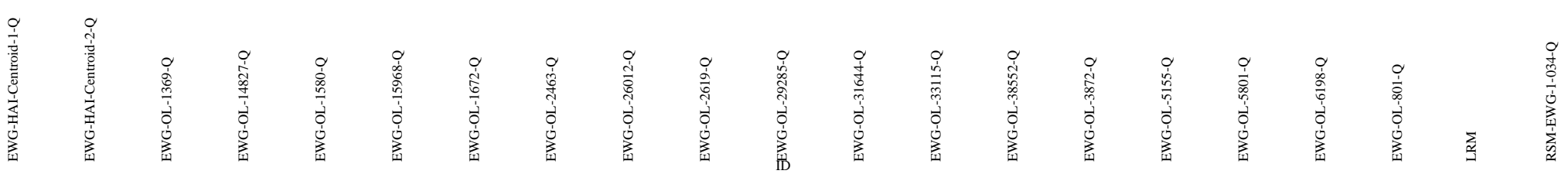

Y

$\bigcirc$ Measured $\quad+{ }^{\text {Targeted }}$ 
SRNL-STI-2014-00063

Revision 0

Appendix B. Tables and Exhibits Supporting the PCT Results 
Table B-1. PCT Measurements for Set 1 Glasses (ar - as received)

\begin{tabular}{|c|c|c|c|c|c|c|c|c|c|c|c|c|c|c|c|c|c|c|c|}
\hline Glass ID & Heat Treatment & Set & Block & Seq & Lab ID & $\begin{array}{c}\text { B } \\
\text { ar }\end{array}$ & $\begin{array}{l}\text { Ca } \\
\text { ar }\end{array}$ & \begin{tabular}{|l|}
$\mathbf{K}$ \\
ar
\end{tabular} & $\begin{array}{l}\mathbf{L i} \\
\mathbf{a r}\end{array}$ & $\begin{array}{l}\text { Na } \\
\text { ar }\end{array}$ & \begin{tabular}{|l|}
$\mathbf{P}$ \\
ar
\end{tabular} & $\begin{array}{l}\text { Si } \\
\text { ar }\end{array}$ & $\begin{array}{c}\text { B } \\
(\mathrm{ppm})\end{array}$ & $\begin{array}{c}\text { Ca } \\
\text { (ppm) }\end{array}$ & $\begin{array}{c}\mathbf{K} \\
(\mathrm{ppm})\end{array}$ & $\begin{array}{c}\mathbf{L i} \\
(\mathbf{p p m})\end{array}$ & $\begin{array}{c}\mathrm{Na} \\
\text { (ppm) }\end{array}$ & $\begin{array}{c}\mathbf{P} \\
(\mathrm{ppm})\end{array}$ & $\begin{array}{c}\mathbf{S i} \\
(\mathbf{p p m})\end{array}$ \\
\hline Std Soln & ref & 1 & 1 & 1 & std-a1-1 & 20.4 & $<1.00$ & 10.4 & 10.6 & 82.1 & $<1.00$ & 50.8 & 20.400 & 1.000 & 10.400 & 10.600 & 82.100 & 1.000 & 50.800 \\
\hline ARM-1 & ref & 1 & 1 & 2 & A58 & 10.0 & $<1.00$ & $<1.00$ & 8.29 & \begin{tabular}{|l|}
20.6 \\
\end{tabular} & $<1.00$ & \begin{tabular}{|l|}
35.6 \\
\end{tabular} & 16.667 & 1.667 & 1.667 & 13.817 & 34.334 & 1.667 & 59.335 \\
\hline blank & ref & 1 & 1 & 3 & A16 & $<1.00$ & $<1.00$ & $<1.00$ & $<1.00$ & $<1.00$ & $<1.00$ & $<1.00$ & 1.667 & 1.667 & 1.667 & 1.667 & 1.667 & 1.667 & 1.667 \\
\hline EWG-OL-2619 & quenched & 1 & 1 & 4 & $\mathrm{~A} 45$ & 26.4 & 5.68 & 8.43 & $<1.00$ & 15.5 & $<1.00$ & 2.55 & 44.001 & 9.467 & 14.050 & 1.667 & 25.834 & 1.667 & 4.250 \\
\hline EWG-OL-2463 & quenched & 1 & 1 & 5 & A57 & 230 & 1.02 & 154 & $\begin{array}{l}<1.00 \\
\end{array}$ & 1150 & $<1.00$ & 14.3 & 383.341 & 1.700 & 256.672 & 1.667 & 1916.705 & 1.667 & 23.834 \\
\hline EWG-OL-2619 & $\mathrm{CCC}$ & 1 & 1 & 6 & A62 & 33.3 & 6.13 & 10.5 & $<1.00$ & \begin{tabular}{|l|}
19.8 \\
\end{tabular} & $<1.00$ & 3.04 & 55.501 & 10.217 & 17.500 & 1.667 & 33.001 & 1.667 & 5.067 \\
\hline EWG-HAI-Centroid-2 & CCC & 1 & 1 & 7 & A33 & 120 & $<1.00$ & 1.94 & 20.2 & 90.1 & $<1.00$ & 22.6 & 200.004 & 1.667 & 3.233 & 33.667 & 150.170 & 1.667 & 37.667 \\
\hline EWG-OL-14827 & quenched & 1 & 1 & 8 & A64 & 28.6 & 3.68 & 9.92 & 13.6 & 17.1 & $<1.00$ & 12.0 & 47.668 & 6.133 & 16.534 & 22.667 & 28.501 & 1.667 & 20.000 \\
\hline EWG-OL-14827 & CCC & 1 & 1 & 9 & $\mathrm{~A} 07$ & 26.5 & 3.89 & 9.00 & 12.5 & 15.4 & $<1.00$ & 11.5 & 44.168 & 6.483 & 15.000 & 20.834 & 25.667 & 1.667 & 19.167 \\
\hline EWG-O & quenched & 1 & 1 & 10 & A66 & 3.64 & 1.46 & $<1.00$ & 5.34 & 3.60 & $<1.00$ & 12.0 & 6.067 & 2.433 & 1.667 & 8.900 & 6.000 & 1.667 & 20.000 \\
\hline EWG-HAI-Ce & uenched & 1 & 1 & 11 & A04 & 109 & $<1.00$ & 1.90 & 18.6 & 82.2 & $<1.00$ & 20.2 & 181.670 & 1.667 & 3.167 & 31.001 & 137.003 & 1.667 & 33.667 \\
\hline EWG-OI & CCC & 1 & 1 & 12 & A21 & 473 & $<1.00$ & 0.524 & $<1.00$ & 603 & $<1.00$ & 22.3 & 788.349 & 1.667 & 0.873 & 260.005 & 1005.020 & 1.667 & 37.167 \\
\hline EWG & quenched & 1 & 1 & 13 & A08 & 59.1 & 4.63 & $<1.00$ & $<1.00$ & 21.8 & $<1.00$ & 3.14 & 98.502 & 7.717 & 1.667 & 1.667 & & 1.667 & 5.233 \\
\hline Std Soln & ref & 1 & 1 & 14 & std-a1-2 & 20.4 & $<1.00$ & 9.82 & 9.93 & 76.2 & $<1.00$ & 48.2 & 20.400 & 1.000 & 9.820 & 9.930 & 76.200 & 1.000 & 48.200 \\
\hline EWG-HAI-Centroid-1 & quenched & 1 & 1 & 15 & A55 & 12.2 & $<1.00$ & 1.27 & 4.29 & 19.7 & $<1.00$ & 19.0 & 20.334 & 1.667 & 2.117 & 7.150 & 32.834 & 1.667 & 31.667 \\
\hline EWG-HAI-Centroid-1 & сCC & 1 & 1 & 16 & A63 & 10.5 & $<1.00$ & 1.06 & 3.31 & 14.8 & $<1.00$ & 12.8 & 17.500 & 1.667 & 1.767 & 5.517 & 24.667 & 1.667 & 21.334 \\
\hline EWG-OL-26012 & quenched & 1 & 1 & 17 & A19 & 7.40 & $<1.00$ & 3.43 & $<1.00$ & 44.1 & 2.09 & 33.0 & 12.334 & 1.667 & 5.717 & 1.667 & 73.501 & 3.483 & 55.001 \\
\hline EWG-C & uenched & 1 & 1 & 18 & A36 & 277 & $<1.00$ & $<1.00$ & 97.8 & 415 & $<1.00$ & 35.9 & 461.676 & 1.667 & 1.667 & 163.003 & 691 & 1.667 & 59.835 \\
\hline EWG & & 1 & 1 & 19 & $\mathrm{~A} 43$ & 8.98 & $<1.00$ & 6.23 & 26.3 & 138 & 79.2 & 43.3 & 14.967 & 1.667 & 10.384 & 43.834 & 230.005 & 132.003 & 72.168 \\
\hline EWG & & 1 & 1 & 20 & A09 & 5.03 & 1.84 & $<1.00$ & 5.39 & 3.59 & $<1.00$ & 11.9 & 8.384 & 3.067 & 1.667 & 8.984 & 5.983 & 1.667 & 19.834 \\
\hline EWG-OL-1580 & $\mathrm{CC}$ & 1 & 1 & 21 & $\mathrm{~A} 10$ & 4.56 & 1.85 & $<1.00$ & 5.12 & 3.78 & $<1.00$ & 12.2 & 7.600 & 3.083 & 1.667 & 8.534 & 6.300 & 1.667 & 20.334 \\
\hline EA & ref & 1 & 1 & 22 & A06 & 19.6 & $<1.00$ & $<1.00$ & 6.89 & 52.1 & $<1.00$ & 35.9 & 326.667 & 16.667 & 16.667 & 114.834 & 868.335 & 16.667 & 598.335 \\
\hline EWG-OL-1580 & quenched & 1 & 1 & 23 & A40 & 4.55 & 1.54 & $<1.00$ & 5.43 & 4.34 & $<1.00$ & 12.8 & \begin{tabular}{|l|}
7.583 \\
\end{tabular} & 2.567 & 1.667 & 9.050 & 7.233 & 1.667 & 21.334 \\
\hline EWG-OL-1672 & CCC & 1 & 1 & 24 & A18 & 61.9 & 4.50 & $<1.00$ & $<1.00$ & 23.3 & $<1.00$ & 3.66 & 103.169 & 7.500 & 1.667 & 1.667 & 38.834 & 1.667 & 6.100 \\
\hline EWG-OL-2463 & CCC & 1 & 1 & 25 & A29 & 503 & 6.97 & 77.5 & $<1.00$ & 2480 & $<1.00$ & 11.2 & 838.350 & 11.617 & 129.169 & 1.667 & 4133.416 & 1.667 & 18.667 \\
\hline Std Soln & ref & 1 & 1 & 26 & std-a1-3 & 22.2 & $<1.00$ & 10.4 & 10.6 & 81.3 & $<1.00$ & 51.1 & 22.200 & 1.000 & 10.400 & 10.600 & \begin{tabular}{|l|}
81.300 \\
\end{tabular} & 1.000 & 51.100 \\
\hline Std So & ref & 1 & 2 & 1 & std-a2-1 & 19.8 & $<1.00$ & 9.78 & 10.2 & 82.4 & $<1.00$ & 48.9 & 19.800 & 1.000 & 9.780 & 10.200 & 82.400 & 1.000 & 48.900 \\
\hline EWG-OL & onch & 1 & 2 & 2 & A25 & 2.44 & $<1.00$ & $<1.00$ & 5.21 & 3.23 & $<1.00$ & 11.1 & 4.067 & 1.667 & 1.667 & 8.684 & 5.383 & 1.667 & 18.500 \\
\hline EWG & nched & 1 & 2 & 3 & A56 & 281 & $<1.00$ & $<1.00$ & 98.1 & 419 & $<1.00$ & 32.5 & 468.343 & 1.667 & 1.667 & 163.503 & 698.347 & 1.667 & 54.168 \\
\hline EWG-HAI-Centroid-1 & enched & 1 & 2 & 4 & A42 & 11.0 & $<1.00$ & $<1.00$ & 4.18 & 20.0 & $<1.00$ & 17.5 & 18.334 & 1.667 & 1.667 & 6.967 & 33.334 & 1.667 & 29.167 \\
\hline EWG-OL-1369 & CCC & 1 & 2 & 5 & A23 & 3.47 & 1.38 & $<1.00$ & 5.39 & 3.22 & $<1.00$ & 11.1 & 5.783 & 2.300 & 1.667 & 8.984 & 5.367 & 1.667 & 18.500 \\
\hline EWG-OL-2619 & CCC & 1 & 2 & 6 & A47 & 23.7 & 4.33 & 7.11 & $<1.00$ & 14.4 & $<1.00$ & 1.58 & 39.501 & 7.217 & 11.850 & 1.667 & 24.000 & 1.667 & 2.633 \\
\hline EWG-OL-2463 & CCC & 1 & 2 & 7 & A03 & 493 & 6.52 & 77.6 & $<1.00$ & 2450 & $<1.00$ & 9.52 & 821.683 & 10.867 & 129.336 & 1.667 & 4083.415 & 1.667 & 15.867 \\
\hline EWG-OL-1580 & $\mathrm{CCC}$ & 1 & 2 & 8 & A52 & 3.73 & 1.31 & $<1.00$ & 4.93 & 3.70 & $<1.00$ & 11.7 & 6.217 & 2.183 & 1.667 & 8.217 & 6.167 & 1.667 & 19.500 \\
\hline EWG-HAI-Centroid-2 & quenched & 1 & 2 & 9 & A20 & 108 & $<1.00$ & 1.18 & 18.1 & 83.4 & $<1.00$ & 19.1 & 180.004 & 1.667 & 1.967 & 30.167 & 139.003 & 1.667 & 31.834 \\
\hline EWG-OL-2619 & quenched & 1 & 2 & 10 & A26 & 25.7 & 5.19 & 7.81 & $<1.00$ & 15.4 & $<1.00$ & 2.36 & 42.834 & 8.650 & 13.017 & 1.667 & 25.667 & 1.667 & 3.933 \\
\hline ARM-1 & ref & 1 & 2 & 11 & A31 & 9.61 & $<1.00$ & $<1.00$ & 8.00 & 20.3 & $<1.00$ & 33.6 & 16.017 & 1.667 & 1.667 & 13.334 & 33.834 & 1.667 & 56.001 \\
\hline EWG-OL-26012 & quenched & 1 & 2 & 12 & A67 & 6.16 & $<1.00$ & 2.61 & $<1.00$ & 43.7 & 2.22 & 30.7 & 10.267 & 1.667 & 4.350 & 1.667 & 72.835 & 3.700 & 51.168 \\
\hline
\end{tabular}


Table B-1. PCT Measurements for Set 1 Glasses (ar - as received) (continued)

\begin{tabular}{|c|c|c|c|c|c|c|c|c|c|c|c|c|c|c|c|c|c|c|c|}
\hline Glass ID & Heat Treatment & Set & Block & Seq & Lab ID & $\begin{array}{l}\text { B } \\
\text { ar }\end{array}$ & $\begin{array}{l}\text { Ca } \\
\text { ar }\end{array}$ & $\begin{array}{l}\mathrm{K} \\
\text { ar }\end{array}$ & $\begin{array}{l}\mathbf{L i} \\
\text { ar }\end{array}$ & $\begin{array}{l}\text { Na } \\
\text { ar }\end{array}$ & $\begin{array}{c}\mathbf{P} \\
\text { ar }\end{array}$ & $\begin{array}{l}\mathrm{Si} \\
\text { ar }\end{array}$ & $\begin{array}{c}\text { B } \\
(\mathbf{p p m})\end{array}$ & $\begin{array}{c}\text { Ca } \\
\text { (ppm) }\end{array}$ & $\begin{array}{c}\mathbf{K} \\
(\mathbf{p p m})\end{array}$ & $\begin{array}{c}\mathbf{L i} \\
(\mathbf{p p m})\end{array}$ & $\begin{array}{c}\text { Na } \\
(\mathbf{p p m})\end{array}$ & $\begin{array}{c}\mathbf{P} \\
(\mathbf{p p m})\end{array}$ & $\begin{array}{c}\mathrm{Si} \\
(\mathbf{p p m})\end{array}$ \\
\hline EA & ref & 1 & 2 & 13 & A14 & 15.8 & $<1.00$ & $<1.00$ & 5.81 & 45.6 & $<1.00$ & 29.7 & 263.334 & 16.667 & 16.667 & 96.834 & 760.002 & 16.667 & 495.001 \\
\hline Std Soln & ref & 1 & 2 & 14 & std-a2-2 & 19.2 & $<1.00$ & 9.45 & 9.90 & 79.2 & $<1.00$ & 47.3 & 19.200 & 1.000 & 9.450 & 9.900 & 79.200 & 1.000 & 47.300 \\
\hline EWG-OL-1672 & $\mathrm{CCC}$ & 1 & 2 & 15 & A22 & 62.2 & 3.85 & $<1.00$ & $<1.00$ & 23.4 & $<1.00$ & 3.42 & 103.669 & 6.417 & 1.667 & 1.667 & 39.001 & 1.667 & 5.700 \\
\hline EWG-HAI-Centroid-1 & CCC & 1 & 2 & 16 & A44 & 9.59 & $<1.00$ & $<1.00$ & 3.33 & 15.4 & $<1.00$ & 12.3 & 15.984 & 1.667 & 1.667 & 5.550 & 25.667 & 1.667 & 20.500 \\
\hline EWG-OL-1580 & quenched & 1 & 2 & 17 & A12 & 3.00 & 1.10 & $<1.00$ & 4.81 & 3.76 & $<1.00$ & 10.8 & 5.000 & 1.833 & 1.667 & 8.017 & 6.267 & 1.667 & 18.000 \\
\hline EWG-OL-15968 & сCC & 1 & 2 & 18 & A54 & 462 & 1.43 & $<1.00$ & 149 & 585 & $<1.00$ & \begin{tabular}{|l|l|}
21.7 \\
\end{tabular} & 770.015 & 2.383 & 1.667 & 248.338 & 975.020 & 1.667 & 36.167 \\
\hline EWG-HAI-Centroid-2 & $\mathrm{CCC}$ & 1 & 2 & 19 & A49 & 123 & $<1.00$ & 1.23 & 19.7 & 91.4 & 1.06 & 21.7 & 205.004 & 1.667 & 2.050 & 32.834 & 152.336 & 1.767 & 36.167 \\
\hline EWG & $\mathrm{CCC}$ & 1 & 2 & 20 & A39 & 7.72 & $<1.00$ & 5.50 & 25.1 & 138 & 74.9 & 40.3 & 12.867 & 1.667 & 9.167 & 41.834 & 230.005 & 124.836 & 67.168 \\
\hline EWC & quenched & 1 & 2 & 21 & A46 & 56.8 & \begin{tabular}{|l|}
4.22 \\
\end{tabular} & $<1.00$ & $<1.00$ & 22.2 & $<1.00$ & 2.76 & 94.669 & 7.033 & 1.667 & 1.667 & 37.001 & \begin{tabular}{|l|}
1.667 \\
\end{tabular} & 4.600 \\
\hline EWG- & CCC & 1 & 2 & 22 & A50 & 25.1 & 3.44 & 8.29 & 12.0 & \begin{tabular}{|l|l|}
15.3 \\
\end{tabular} & $<1.00$ & 10.5 & 41.834 & 5.733 & 13.817 & 20.000 & 25.501 & 1.667 & 17.500 \\
\hline EWG-OL- & quenched & 1 & 2 & 23 & A28 & 212 & $<1.00$ & 144 & $<1.00$ & 1060 & $<1.00$ & 14.2 & 353.340 & 1.667 & 240.005 & 1.667 & 1766.702 & 1.667 & 23.667 \\
\hline EWG-OL-14827 & quenched & 1 & 2 & 24 & A59 & 29.3 & 3.30 & 9.56 & 13.4 & \begin{tabular}{|l|}
17.3 \\
\end{tabular} & $<1.00$ & 11.3 & 48.834 & 5.500 & 15.934 & 22.334 & 28.834 & 1.667 & 18.834 \\
\hline Std Soln & ref & 1 & 2 & 25 & std-a2-3 & 20.5 & $<1.00$ & 9.79 & 10.3 & 82.3 & $<1.00$ & \begin{tabular}{|l|}
49.1 \\
\end{tabular} & 20.500 & 1.000 & 9.790 & 10.300 & 82.300 & 1.000 & 49.100 \\
\hline Std Soln & ref & 1 & 3 & 1 & std-a3-1 & 20.0 & $<1.00$ & 9.90 & 10.3 & 80.9 & $<1.00$ & 48.5 & 20.000 & 1.000 & 9.900 & 10.300 & 80.900 & 1.000 & 48.500 \\
\hline EWG-OL-2619 & quenched & 1 & 3 & 2 & A51 & 26.4 & 5.55 & 8.31 & $<1.00$ & 15.8 & $<1.00$ & 1.72 & 44.001 & 9.250 & 13.850 & 1.667 & 26.334 & 1.667 & 2.867 \\
\hline EWC & & 1 & 3 & 3 & & 25.3 & 3.60 & 8.57 & 12.2 & 15.4 & $<1.00$ & 10.1 & 42.168 & 6.000 & 14.284 & 20.334 & 25.667 & 1.667 & 16.834 \\
\hline EWG-C & & 1 & 3 & 4 & $\mathrm{~A} 48$ & 7.35 & $<1.00$ & 5.86 & 26.1 & 139 & 76.6 & 40.7 & 12.250 & 1.667 & 9.767 & 43.501 & 231.671 & 127.669 & 67.835 \\
\hline blank & & 1 & 3 & 5 & A37 & $<1.00$ & $<1.00$ & $<1.00$ & $<1.00$ & $<1.00$ & $<1.00$ & $<1.00$ & 1.667 & 1.667 & 1.667 & 1.667 & 1.667 & 1.667 & 1.667 \\
\hline EWG-OL-1580 & quenched & 1 & 3 & 6 & A34 & 3.04 & 1.26 & $<1.00$ & 5.27 & \begin{tabular}{|l|}
3.94 \\
\end{tabular} & $<1.00$ & 11.6 & 5.067 & 2.100 & 1.667 & 8.784 & 6.567 & 1.667 & 19.334 \\
\hline EWG-OL-1369 & quenched & 1 & 3 & 7 & A13 & 2.70 & 1.13 & $<1.00$ & 5.55 & 3.43 & $<1.00$ & 11.6 & 4.500 & 1.883 & 1.667 & 9.250 & 5.717 & 1.667 & 19.334 \\
\hline EWG-OL-15968 & quenched & 1 & 3 & 8 & A02 & 295 & $<1.00$ & $<1.00$ & 98.6 & 422 & $<1.00$ & 31.9 & 491.677 & 1.667 & 1.667 & 164.337 & 703.347 & 1.667 & 53.168 \\
\hline EWG-OL-2463 & сCC & 1 & 3 & 9 & A32 & 484 & 6.70 & 78.4 & $<1.00$ & 2490 & $<1.00$ & 10.0 & 806.683 & 11.167 & 130.669 & 1.667 & 4150.083 & 1.667 & 16.667 \\
\hline EWG-HAI-Centroid-1 & $\mathrm{CCC}$ & 1 & 3 & 10 & A60 & 10.3 & $<1.00$ & $<1.00$ & \begin{tabular}{|l|}
3.34 \\
\end{tabular} & \begin{tabular}{|l|}
15.2 \\
\end{tabular} & $<1.00$ & 12.0 & 17.167 & 1.667 & \begin{tabular}{|l}
1.667 \\
\end{tabular} & 5.567 & 25.334 & 1.667 & 20.000 \\
\hline & ref & 1 & 3 & 11 & A30 & 16.2 & $<1.00$ & $<1.00$ & 5.87 & 45.6 & $<1.00$ & 29.5 & 270.001 & 16.667 & 16.667 & 97.834 & 760.002 & 16.667 & 491.668 \\
\hline EWG-O & denche & 1 & 3 & 12 & 41 & 57.7 & \begin{tabular}{|l|}
4.39 \\
\end{tabular} & $<1.00$ & $<1.00$ & 22.4 & $<1.00$ & 2.54 & \begin{tabular}{|l|}
96.169 \\
\end{tabular} & 7.317 & 1.667 & 1.667 & 37.334 & 1.667 & 4.233 \\
\hline EWG- & & 1 & 3 & 13 & A27 & 447 & $<1.00$ & $<1.00$ & 145 & 568 & $<1.00$ & 22.4 & 745.015 & 1.667 & 1.667 & 241.672 & 946.686 & 1.667 & 37.334 \\
\hline St & & 1 & 3 & 14 & std-a3-2 & 20.8 & $<1.00$ & 9.91 & 10.3 & 81.2 & $<1.00$ & 48.3 & 20.800 & 1.000 & 9.910 & 10.300 & 81.200 & 1.000 & 48.300 \\
\hline EWG-OL-14827 & uenched & 1 & 3 & 15 & A38 & 29.3 & 3.41 & 9.71 & 13.6 & 17.3 & $<1.00$ & 10.7 & 48.834 & 5.683 & 16.184 & 22.667 & 28.834 & 1.667 & 17.834 \\
\hline EWG-OL-26012 & quenched & 1 & 3 & 16 & A65 & 6.57 & $<1.00$ & 2.72 & $<1.00$ & 43.3 & 2.06 & 30.5 & 10.950 & 1.667 & 4.533 & 1.667 & 72.168 & 3.433 & 50.834 \\
\hline EWG-OL-1369 & CCC & 1 & 3 & 17 & A61 & 4.22 & 1.56 & $<1.00$ & 5.48 & 3.45 & $<1.00$ & 11.5 & 7.033 & 2.600 & 1.667 & 9.134 & 5.750 & 1.667 & 19.167 \\
\hline EWG-HAI-Centroid-2 & CCC & 1 & 3 & 18 & A15 & 126 & $<1.00$ & 1.53 & 20.4 & 93.5 & $<1.00$ & 21.8 & 210.004 & 1.667 & 2.550 & 34.001 & 155.836 & 1.667 & 36.334 \\
\hline EWG-HAI-Centroid-1 & quenched & 1 & 3 & 19 & A05 & 11.3 & $<1.00$ & $<1.00$ & 4.25 & 19.9 & $<1.00$ & \begin{tabular}{|l|}
17.1 \\
\end{tabular} & 18.834 & 1.667 & 1.667 & 7.083 & 33.167 & 1.667 & 28.501 \\
\hline ARM-1 & ref & 1 & 3 & 20 & A68 & 10.1 & $<1.00$ & $<1.00$ & 8.32 & 20.9 & $<1.00$ & 34.5 & 16.834 & 1.667 & 1.667 & 13.867 & 34.834 & 1.667 & 57.501 \\
\hline EWG-HAI-Centroid-2 & quenched & 1 & 3 & 21 & A24 & 109 & $<1.00$ & 1.43 & 18.3 & 83.6 & $<1.00$ & 18.8 & 181.670 & 1.667 & 2.383 & 30.501 & 139.336 & 1.667 & 31.334 \\
\hline EWG-OL-1580 & CCC & 1 & 3 & 22 & A11 & 3.71 & 1.54 & $<1.00$ & 5.22 & 3.57 & $<1.00$ & 11.2 & \begin{tabular}{|l|}
6.183 \\
\end{tabular} & 2.567 & 1.667 & 8.700 & 5.950 & 1.667 & 18.667 \\
\hline EWG-OL-1672 & $\mathrm{CCC}$ & 1 & 3 & 23 & 17 & 63.8 & 4.01 & $<1.00$ & $<1.00$ & 23.8 & $<1.00$ & 2.33 & 106.335 & 6.683 & 1.667 & 1.667 & 39.667 & 1.667 & 3.883 \\
\hline & & 1 & 3 & 24 & A35 & 33.3 & 5.97 & 10.4 & $<1.00$ & 20.0 & $<1.00$ & 2.13 & 55.501 & 9.950 & 17.334 & 1.667 & & 1.667 & 3.550 \\
\hline EWG-OL-2463 & quenched & 1 & 3 & 25 & A01 & 226 & $<1.00$ & 149 & $<1.00$ & 1120 & $<1.00$ & 13.2 & 376.674 & 1.667 & 248.338 & 1.667 & 1866.704 & 1.667 & 22.000 \\
\hline
\end{tabular}


Table B-1. PCT Measurements for Set 1 Glasses (ar - as received) (continued)

\begin{tabular}{|c|c|c|c|c|c|c|c|c|c|c|c|c|c|c|c|c|c|c|c|}
\hline Glass ID & Heat Treatment & Set & Block & Seq & Lab ID & $\begin{array}{l}\text { B } \\
\text { ar }\end{array}$ & $\begin{array}{l}\text { Ca } \\
\text { ar }\end{array}$ & $\begin{array}{l}\text { K } \\
\text { ar }\end{array}$ & $\begin{array}{l}\mathbf{L i} \\
\text { ar }\end{array}$ & $\begin{array}{l}\text { Na } \\
\text { ar }\end{array}$ & $\begin{array}{l}\mathbf{P} \\
\text { ar }\end{array}$ & $\begin{array}{l}\mathrm{Si} \\
\text { ar }\end{array}$ & $\begin{array}{c}\text { B } \\
(\mathrm{ppm})\end{array}$ & $\begin{array}{c}\text { Ca } \\
\text { (ppm) }\end{array}$ & $\begin{array}{c}\mathbf{K} \\
(\mathbf{p p m})\end{array}$ & $\begin{array}{c}\mathbf{L i} \\
(\mathbf{p p m})\end{array}$ & $\begin{array}{c}\text { Na } \\
\text { (ppm) }\end{array}$ & $\begin{array}{c}\mathbf{P} \\
(\mathbf{p p m})\end{array}$ & $\begin{array}{c}\mathrm{Si} \\
\text { (ppm) }\end{array}$ \\
\hline Std Soln & ref & 1 & 3 & 26 & std-a3-3 & 20.7 & $<1.00$ & 10.1 & 10.4 & 83.0 & $<1.00$ & 49.4 & 20.700 & 1.000 & 10.100 & 10.400 & 83.000 & 1.000 & 49.400 \\
\hline Std Soln & ref & 2 & 1 & 1 & std-b1-1 & $\mid 19.4$ & $<1.00$ & 9.94 & 10.2 & 79.1 & $<1.00$ & 48.1 & 19.400 & .000 & 9.940 & 10.200 & 99.100 & 1.000 & \\
\hline EWG-OL-33115 & $\mathrm{CCC}$ & 2 & 1 & 2 & B10 & 4.44 & $<1.00$ & 3.08 & $<1.00$ & \begin{tabular}{|l|}
10.6 \\
\end{tabular} & $<1.00$ & 13.7 & 7.400 & \begin{tabular}{|l|l}
1.667 \\
\end{tabular} & 5.133 & 1.667 & 17.667 & 1.667 & .834 \\
\hline EWG-OL- & quenched & 2 & 1 & 3 & B25 & 20.9 & 1.86 & $<1.00$ & 28.7 & 171 & $<1.00$ & 46.6 & 34.834 & 2.900 & 1.667 & 47.834 & 285.006 & 1.667 & 77.668 \\
\hline EWG-OL-33115 & uenched & 2 & 1 & 4 & B35 & 3.79 & 1.65 & 3.13 & $<1.00$ & 11.3 & $<1.00$ & 10.6 & 6.317 & 2.550 & 5.217 & 1.667 & 18.834 & 1.667 & 17.667 \\
\hline EWG-OL-38552 & $\mathrm{ccc}$ & 2 & 1 & 5 & B37 & 240 & $<1.00$ & $<1.00$ & 44.4 & 220 & 2.43 & 4.38 & 400.008 & 1.667 & 1.667 & 74.001 & 366.674 & 4.050 & 7.300 \\
\hline EWG-OL-6198 & CCC & 2 & 1 & 6 & B17 & 253 & $<1.00$ & 15.2 & 78.2 & 81.9 & 12.7 & 20.9 & 421.675 & 1.667 & 25.334 & 130.336 & \begin{tabular}{|l|}
136.503 \\
\end{tabular} & 21.167 & 34.834 \\
\hline EWG & uenc & 2 & 1 & 7 & B04 & 11.3 & $<1.00$ & $<1.00$ & 12.6 & 8.55 & 4.39 & 21.2 & 18.834 & .667 & 1.667 & 21.000 & & 7.317 & 5.334 \\
\hline EWG & & 2 & 1 & $\varepsilon$ & & 81.8 & $<1.00$ & $<1.00$ & 32.9 & 37.0 & $<1.00$ & 14.7 & 136.336 & 667 & 1.667 & 54.834 & & 1.667 & 4.500 \\
\hline ARN & $\mathrm{r}$ & 2 & $\mathrm{I}$ & $\mathrm{s}$ & $\mathrm{B}$ & 10.5 & $<1.00$ & $<1.00$ & 7.60 & 19.0 & 1.11 & 32.3 & 17.500 & 217 & 1.667 & 12.667 & 667 & 1.850 & 8.834 \\
\hline EWG-C & nched & 2 & . & 10 & B52 & 217 & $<1.00$ & $<1.00$ & $<1.00$ & 373 & 13.7 & 12.9 & 361.674 & .667 & 1.667 & \begin{tabular}{|l|}
1.667 \\
\end{tabular} & 679 & 22.834 & .500 \\
\hline RSM-E & & 2 & 1 & 11 & B43 & 16.0 & $<1.00$ & $<1.00$ & 4.55 & \begin{tabular}{|l|}
17.3 \\
\end{tabular} & 1.59 & 13.7 & 667 & 1.667 & 1.667 & 7.583 & & 2.650 & .834 \\
\hline EWG-O & uenched & 2 & 1 & 12 & B50 & 82.2 & $<1.00$ & \begin{tabular}{|l|}
10.1 \\
\end{tabular} & 33.4 & 35.7 & 4.40 & 17.3 & 137.003 & 1.667 & 16.834 & 55.668 & 59.501 & 7.333 & 28.834 \\
\hline EWG-OL-801 & & 2 & 1 & 13 & B01 & 256 & $<1.00$ & $<1.00$ & $<1.00$ & 410 & 17.9 & 13.3 & 426.675 & 1.667 & 1.667 & 1.667 & 683.347 & 29.834 & 22.167 \\
\hline & & 2 & 1 & 14 & std-b1-2 & 21.2 & $<1.00$ & 9.92 & 10.1 & 80.4 & $<1.00$ & 46.9 & 21.200 & 1.000 & 9.920 & 10.100 & & 1.000 & 46.900 \\
\hline EWG-OL-5801 & & 2 & 1 & 15 & & 97.8 & $<1.00$ & 17.7 & 39.1 & 48.0 & 8.94 & 12.0 & & 1.667 & 29.501 & & & 14.900 & 20.000 \\
\hline EWG & & 2 & 1 & 1 & & 7.10 & 2.45 & $<1.00$ & $<1.00$ & 4.88 & $<1.00$ & 6.32 & & .783 & 1.667 & & & 1.667 & 0.534 \\
\hline EWG- & & 2 & 1 & 1 & $\mathrm{H}$ & 265 & 21.9 & $<1.00$ & 107 & 104 & $<1.00$ & $<1.00$ & & 1.667 & \begin{tabular}{|l|}
1.667 \\
\end{tabular} & 178.337 & & 1.667 & \\
\hline $\mathrm{E}$ & & 2 & 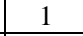 & 18 & B62 & 32.4 & $<1.00$ & $<1.00$ & 10.9 & 88.2 & $<1.00$ & 48.0 & 540.001 & 16.667 & 16.667 & 181.667 & 1470.003 & 16.667 & 300.002 \\
\hline EWG-OL & & 2 & 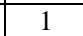 & 19 & B46 & 7.21 & 2.28 & $<1.00$ & $<1.00$ & 4.53 & $<1.00$ & 6.96 & 12.017 & 3.433 & \begin{tabular}{|l|}
1.667 \\
\end{tabular} & \begin{tabular}{|l|}
1.667 \\
\end{tabular} & 7.550 & 1.667 & 11.600 \\
\hline EWG-C & & 2 & 1 & 20 & $\mathrm{E}$ & 4.96 & 1.58 & $<1.00$ & 2.02 & 3.43 & $<1.00$ & 9.83 & 267 & 2.367 & 1.667 & 3.367 & .717 & 1.667 & 16.384 \\
\hline blank & ref & 2 & 1 & 21 & B45 & 1.65 & $<1.00$ & $<1.00$ & $<1.00$ & $<1.00$ & $<1.00$ & $<1.00$ & 2.750 & 1.667 & 1.667 & 1.667 & 1.667 & 1.667 & 1.667 \\
\hline EWG-OL-3872 & & 2 & 1 & 22 & B51 & 600 & \begin{tabular}{|l|}
1.22 \\
\end{tabular} & $<1.00$ & 268 & 2210 & \begin{tabular}{|l|}
2.38 \\
\end{tabular} & 117 & 1000.020 & 1.783 & 1.667 & 446.676 & 3683.407 & 3.967 & 195.004 \\
\hline RSM-EWG-1-034 & & 2 & 1 & 23 & & 16.8 & $<1.00$ & $<1.00$ & 4.79 & 15.8 & 1.43 & 10.6 & & 1.667 & 1.667 & 7.983 & & 2.383 & 17.667 \\
\hline EWG & & 2 & 1 & 24 & & 227 & $<1.00$ & \begin{tabular}{|l|}
26.8 \\
\end{tabular} & \begin{tabular}{|l}
74.9 \\
\end{tabular} & 105 & 7.08 & 1.11 & & .667 & 44.668 & 124.836 & & 11.800 & .850 \\
\hline EWG-O & nch & 2 & 1 & 25 & 306 & 6.37 & 3.64 & $<1.00$ & $<1.00$ & 5.33 & $<1.00$ & 9.13 & 617 & 5.783 & 1.667 & 1.667 & 8.884 & 1.667 & 15.217 \\
\hline & & 2 & 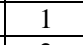 & 26 & std-b1-3 & 22.0 & $<1.00$ & \begin{tabular}{|l|}
10.3 \\
\end{tabular} & 10.5 & 83.8 & $<1.00$ & 48.1 & 000 & 1.000 & 10.300 & 10.500 & 800 & 1.000 & 48.100 \\
\hline & & 2 & 2 & 1 & std-b2-1 & 20.5 & $<1.00$ & 10.4 & 10.6 & 82.2 & $<1.00$ & 49.8 & 20.500 & 1.000 & 10.400 & 10.600 & 82.200 & 1.000 & 49.800 \\
\hline EWG-OL-29285 & & 2 & 2 & 2 & B26 & 5.39 & 2.29 & $<1.00$ & $<1.00$ & 4.53 & $<1.00$ & 6.71 & 8.984 & 3.417 & \begin{tabular}{|l|}
1.667 \\
\end{tabular} & 1.667 & 7.550 & 1.667 & 11.184 \\
\hline EWG-OL-3872 & uenche & 2 & 2 & 3 & $\mathrm{~B}$ & 21.1 & 1.73 & $<1.00$ & 28.8 & 171 & $<1.00$ & 45.2 & 35.167 & 2.400 & 1.667 & 48.001 & 285.006 & 1.667 & 5.335 \\
\hline EWG-OL-3872 & & 2 & 2 & 4 & 354 & 596 & 1.09 & $<1.00$ & 265 & 2180 & 2.32 & 119 & 93.353 & 1.667 & 1.667 & 441.676 & 3633.406 & 3.867 & 198.337 \\
\hline EWG-OL-6198 & quenched & 2 & 2 & 5 & $\mathrm{~B} 21$ & 92.7 & $<1.00$ & 10.4 & 34.8 & 36.6 & 4.37 & 17.9 & 154.503 & 1.667 & 17.334 & 58.001 & \begin{tabular}{|l|}
61.001 \\
\end{tabular} & 7.283 & 29.834 \\
\hline EWG-OL-31644 & quenched & 2 & 2 & 6 & B22 & 4.10 & \begin{tabular}{|l|}
3.47 \\
\end{tabular} & $<1.00$ & $<1.00$ & 5.27 & $<1.00$ & 8.42 & 6.833 & 5.500 & \begin{tabular}{|l|}
1.667 \\
\end{tabular} & 1.667 & & 1.667 & 14.034 \\
\hline EWG-OL-31644 & & 2 & 2 & 7 & B11 & 4.05 & 1.42 & $<1.00$ & 2.01 & 3.40 & $<1.00$ & 9.35 & 6.750 & 1.933 & 1.667 & 3.350 & & 1.667 & 15.584 \\
\hline EWG-OL & ncnea & 2 & 2 & 8 & B66 & 96.7 & $<1.00$ & 17.7 & 38.6 & 46.7 & \begin{tabular}{|l|}
8.88 \\
\end{tabular} & 12.0 & 161.170 & 1.667 & 29.501 & 64.335 & 77.835 & 14.800 & 20.000 \\
\hline EWG-OL-38552 & quencned & 2 & 2 & 9 & $\mathrm{~B}$ & 9.67 & $<1.00$ & $<1.00$ & 12.9 & 8.76 & 4.28 & 20.2 & 5.117 & 1.667 & 1.667 & 21.500 & 4.600 & 7.133 & \begin{tabular}{|l|l}
33.667 \\
\end{tabular} \\
\hline EWG-OL-5155 & & 2 & 2 & 10 & & 287 & $<1.00$ & $<1.00$ & 111 & 105 & $<1.00$ & $<1.00$ & 3.343 & 1.667 & 1.667 & 185.004 & & 1.667 & 1.667 \\
\hline & $\mathrm{r}$ & 2 & 2 & 11 & B58 & 33.8 & $<1.00$ & $<1.00$ & 11.1 & 91.7 & $<1.00$ & 49.1 & 3.334 & 6.667 & 16.667 & 185.000 & 1528.336 & 16.667 & 318.335 \\
\hline 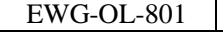 & $\operatorname{ccc}+y_{1}$ & 2 & 2 & 12 & B53 & 249 & 27.8 & 1.00 & & 409 & 18.7 & 12.9 & 415.008 & $1.06 / \mathrm{s}$ & 1.667 & 1.667 & 681.680 & & 21.500 \\
\hline
\end{tabular}


Table B-1. PCT Measurements for Set 1 Glasses (ar - as received) (continued)

\begin{tabular}{|c|c|c|c|c|c|c|c|c|c|c|c|c|c|c|c|c|c|c|c|}
\hline Glass ID & Heat Treatment & Set & Block & Seq & Lab ID & $\begin{array}{c}\text { B } \\
\text { ar }\end{array}$ & $\begin{array}{l}\text { Ca } \\
\text { ar }\end{array}$ & $\begin{array}{l}\text { K } \\
\text { ar }\end{array}$ & $\begin{array}{l}\mathbf{L i} \\
\text { ar }\end{array}$ & $\begin{array}{l}\mathrm{Na} \\
\text { ar }\end{array}$ & $\begin{array}{c}\mathbf{P} \\
\text { ar }\end{array}$ & $\begin{array}{l}\mathrm{Si} \\
\text { ar }\end{array}$ & $\begin{array}{c}\text { B } \\
(\mathrm{ppm})\end{array}$ & $\begin{array}{c}\text { Ca } \\
\text { (ppm) }\end{array}$ & $\begin{array}{c}\mathbf{K} \\
(\mathbf{p p m})\end{array}$ & $\begin{array}{c}\mathbf{L i} \\
(\mathbf{p p m})\end{array}$ & $\begin{array}{c}\text { Na } \\
\text { (ppm) }\end{array}$ & $\begin{array}{c}\mathbf{P} \\
(\mathrm{ppm})\end{array}$ & $\begin{array}{c}\mathrm{Si} \\
(\mathbf{p p m})\end{array}$ \\
\hline EWG-OL-33115 & quenched & 2 & 2 & 13 & B19 & 5.03 & 1.59 & 3.18 & $<1.00$ & 11.4 & $<1.00$ & 9.06 & 8.384 & 2.083 & 5.300 & 1.667 & 19.000 & 1.667 & 15.100 \\
\hline Std Soln & ref & 2 & 2 & 14 & \begin{tabular}{|l|} 
std-b2-2 \\
\end{tabular} & 20.6 & $<1.00$ & 10.3 & 10.4 & 81.6 & $<1.00$ & 48.4 & 20.600 & 1.000 & 10.300 & 10.400 & 81.600 & 1.000 & 48.400 \\
\hline EWG-OL-5801 & CCC & 2 & 2 & 15 & B07 & 221 & $<1.00$ & 26.0 & 72.7 & 99.0 & 7.12 & $<1.00$ & 368.341 & 1.667 & 43.334 & 121.169 & 165.003 & 11.867 & 1.667 \\
\hline EWG-OL-29285 & quenched & 2 & 2 & 16 & B56 & 7.05 & 2.33 & $<1.00$ & $<1.00$ & 4.61 & $<1.00$ & 6.37 & 11.750 & 3.533 & 1.667 & 1.667 & 7.683 & 1.667 & 10.617 \\
\hline EWG-OL-5155 & quenched & 2 & 2 & 17 & B38 & 83.3 & $<1.00$ & $<1.00$ & 33.1 & 37.3 & $<1.00$ & 13.4 & 138.836 & 1.667 & 1.667 & 55.168 & 62.168 & 1.667 & 22.334 \\
\hline EWG-OL-38552 & CCC & 2 & 2 & 18 & B27 & 239 & $<1.00$ & $<1.00$ & 45.2 & 216 & 1.80 & $<1.00$ & 398.341 & 1.667 & 1.667 & 75.335 & 360.007 & 3.000 & 1.667 \\
\hline ARM-1 & ref & 2 & 2 & 19 & B63 & 10.9 & $<1.00$ & $<1.00$ & 7.73 & 19.3 & $<1.00$ & 32.4 & 18.167 & 1.667 & 1.667 & 12.884 & 32.167 & 1.667 & 54.001 \\
\hline RSM-EWG-1-034 & quenched & 2 & 2 & 20 & B48 & 13.8 & $<1.00$ & $<1.00$ & 4.81 & 15.2 & 1.23 & 11.0 & 23.000 & 1.667 & 1.667 & 8.017 & 25.334 & 2.050 & 18.334 \\
\hline EWG-OL-6198 & CCC & 2 & 2 & 21 & B18 & 261 & $<1.00$ & 15.5 & 79.1 & 83.5 & 11.3 & 19.7 & 435.009 & 1.667 & 25.834 & 131.836 & 139.169 & 18.834 & 32.834 \\
\hline RSM-EWG-1-034 & CCC & 2 & 2 & 22 & B16 & 16.1 & $<1.00$ & $<1.00$ & 4.56 & 17.5 & 1.44 & 12.9 & 26.834 & 1.667 & 1.667 & \begin{tabular}{|l|}
7.600 \\
\end{tabular} & 29.167 & 2.400 & 21.500 \\
\hline EWG-OL-801 & quenched & 2 & 2 & 23 & B09 & 227 & $<1.00$ & $<1.00$ & $<1.00$ & 365 & 14.0 & 12.4 & 378.341 & 1.667 & 1.667 & 1.667 & 608.346 & 23.334 & 20.667 \\
\hline EWG-OL-33115 & CCC & 2 & 2 & 24 & B20 & 5.78 & $<1.00$ & 3.11 & $<1.00$ & 10.4 & $<1.00$ & 12.1 & 9.634 & 1.667 & 5.183 & 1.667 & 17.334 & 1.667 & 20.167 \\
\hline Std Soln & ref & 2 & 2 & 25 & std-b2-3 & 21.1 & $<1.00$ & 10.4 & 10.4 & 81.3 & $<1.00$ & 49.0 & 21.100 & 1.000 & 10.400 & 10.400 & 81.300 & 1.000 & 49.000 \\
\hline Std Soln & ref & 2 & 3 & 1 & std-b3-1 & 19.9 & $<1.00$ & 10.1 & 10.2 & 80.5 & $<1.00$ & 48.2 & 19.900 & 1.000 & 10.100 & 10.200 & 80.500 & 1.000 & 48.200 \\
\hline EWG-OL & quenched & 2 & 3 & 2 & B24 & 8.74 & $<1.00$ & $<1.00$ & 12.8 & 8.66 & 4.18 & 20.3 & 14.567 & 1.667 & 1.667 & 21.334 & 14.434 & 6.967 & 33.834 \\
\hline EWG-OL-801 & CCC & 2 & 3 & 3 & B02 & 268 & 40.1 & $<1.00$ & $<1.00$ & 420 & 18.7 & 12.7 & 446.676 & 1.667 & 1.667 & 1.667 & 700.014 & 31.167 & 21.167 \\
\hline EWG-OL-5801 & quenched & 2 & 3 & 4 & B55 & 102 & $<1.00$ & 17.8 & 38.3 & 46.7 & 9.22 & 12.3 & 170.003 & 1.667 & 29.667 & 63.835 & 77.835 & 15.367 & 20.500 \\
\hline ARM-1 & ref & 2 & 3 & 5 & B39 & 9.77 & $<1.00$ & $<1.00$ & 7.66 & 19.2 & $<1.00$ & 31.8 & 16.284 & 1.667 & 1.667 & 12.767 & 32.001 & 1.667 & 53.001 \\
\hline EA & ref & 2 & 3 & 6 & B65 & 32.8 & 1.02 & $<1.00$ & 11.5 & 92.2 & $<1.00$ & 51.6 & 546.668 & 16.667 & 16.667 & 191.667 & 1536.670 & 16.667 & 860.002 \\
\hline EWG-OL-6198 & CCC & 2 & 3 & 7 & B08 & 263 & $<1.00$ & 15.5 & 79.0 & 85.3 & 12.0 & 19.8 & 438.342 & 1.667 & 25.834 & 131.669 & 142.170 & 20.000 & 33.001 \\
\hline EWG-OL- & CCC & 2 & 3 & 8 & B05 & 292 & $<1.00$ & $<1.00$ & 111 & 105 & $<1.00$ & $<1.00$ & 486.676 & 1.667 & 1.667 & 185.004 & 175.004 & 1.667 & 1.667 \\
\hline EWG-OL-29285 & CCC & 2 & 3 & 9 & B68 & 6.58 & 2.12 & $<1.00$ & $<1.00$ & 7.86 & $<1.00$ & 6.43 & 10.967 & 3.250 & 1.667 & \begin{tabular}{|l|}
1.667 \\
\end{tabular} & 13.100 & 1.667 & 10.717 \\
\hline EWG-OL-5801 & c & 2 & 3 & 10 & B59 & 229 & $<1.00$ & 26.5 & 72.8 & 101 & 6.87 & $<1.00$ & 381.674 & 1.667 & 44.168 & 121.336 & 168.337 & 11.450 & 1.667 \\
\hline EWG-OL & & 2 & 3 & 11 & B29 & 253 & $<1.00$ & $<1.00$ & 45.0 & 217 & 2.49 & 3.87 & 421.675 & 1.667 & 1.667 & \begin{tabular}{|l|}
75.002 \\
\end{tabular} & 361.674 & 4.150 & 6.450 \\
\hline EWG-OL-801 & uenched & 2 & 3 & 12 & B12 & 225 & $<1.00$ & $<1.00$ & $<1.00$ & 381 & 14.5 & 12.4 & 375.008 & 1.667 & 1.667 & 1.667 & 635.013 & 24.167 & 20.667 \\
\hline EWG-OL-33115 & quenched & 2 & 3 & 13 & B42 & 5.62 & 20.8 & 3.02 & $<1.00$ & 12.0 & $<1.00$ & 9.13 & 9.367 & 2.017 & 5.033 & 1.667 & 20.000 & 1.667 & 15.217 \\
\hline Std Soln & ref & 2 & 3 & 14 & std-b3-2 & 21.2 & $<1.00$ & 10.1 & 10.4 & 81.8 & $<1.00$ & \begin{tabular}{|l|l|}
48.4 & \\
\end{tabular} & 21.200 & 1.000 & 10.100 & 10.400 & 81.800 & 1.000 & 48.400 \\
\hline EWG-OL-3872 & CCC & 2 & 3 & 15 & B67 & 564 & 1.01 & $<1.00$ & 256 & 2110 & 2.39 & 124 & 940.019 & 1.667 & 1.667 & 426.675 & 3516.737 & 3.983 & 206.671 \\
\hline EWG-OL-29285 & quenched & 2 & 3 & 16 & B61 & 8.53 & 2.26 & $<1.00$ & $<1.00$ & 4.93 & $<1.00$ & 6.14 & 14.217 & 3.400 & 1.667 & \begin{tabular}{|l|}
1.667 \\
\end{tabular} & 8.217 & 1.667 & 10.234 \\
\hline EWG-OL-31644 & quenched & 2 & 3 & 17 & $\mathrm{~B} 40$ & 4.65 & 3.54 & $<1.00$ & $<1.00$ & 5.35 & $<1.00$ & 8.27 & 7.750 & 5.383 & 1.667 & 1.667 & 8.917 & 1.667 & 13.784 \\
\hline EWG-OL-3872 & quenched & 2 & 3 & 18 & B23 & 21.7 & 1.84 & $<1.00$ & 27.7 & 167 & $<1.00$ & 43.5 & 36.167 & 2.433 & 1.667 & 46.168 & 278.339 & 1.667 & 72.501 \\
\hline EWG-OL-5155 & quenched & 2 & 3 & 19 & B64 & 88.3 & $<1.00$ & $<1.00$ & 34.4 & 39.1 & $<1.00$ & 14.0 & 147.170 & 1.667 & 1.667 & 57.334 & 65.168 & 1.667 & 23.334 \\
\hline RSM-EWG-1-034 & CCC & 2 & 3 & 20 & B57 & 16.5 & $<1.00$ & $<1.00$ & 4.74 & 17.9 & 1.31 & 13.1 & 27.501 & 1.667 & 1.667 & 7.900 & 29.834 & 2.183 & 21.834 \\
\hline blank & ref & 2 & 3 & 21 & B30 & $<1.00$ & $<1.00$ & $<1.00$ & $<1.00$ & $<1.00$ & $<1.00$ & $<1.00$ & 1.667 & 1.667 & 1.667 & 1.667 & 1.667 & 1.667 & 1.667 \\
\hline EWG-OL-6198 & quenched & 2 & 3 & 22 & B33 & 90.8 & $<1.00$ & 10.4 & 34.7 & 36.8 & 4.47 & 17.5 & 151.336 & 1.667 & 17.334 & 57.834 & 61.335 & 7.450 & 29.167 \\
\hline EWG-OL-33115 & CCC & 2 & 3 & 23 & B60 & 4.65 & $<1.00$ & 2.93 & $<1.00$ & 10.5 & $<1.00$ & 11.8 & 7.750 & 1.667 & 4.883 & 1.667 & 17.500 & 1.667 & 19.667 \\
\hline RSM-EWG-1-034 & quenched & 2 & 3 & 24 & B03 & 13.5 & $<1.00$ & $<1.00$ & 4.82 & 15.2 & 1.12 & 10.7 & 22.500 & 1.667 & 1.667 & 8.033 & 25.334 & 1.867 & 17.834 \\
\hline EWG-OL-31644 & сCC & 2 & 3 & 25 & B31 & 3.31 & 76.8 & $<1.00$ & 1.91 & 3.61 & $<1.00$ & 8.65 & 5.517 & 1.900 & 1.667 & 3.183 & 6.017 & 1.667 & 14.417 \\
\hline Std Soln & ref & 2 & 3 & 26 & std-b3-3 & 19.2 & $<1.00$ & \begin{tabular}{|l}
9.87 \\
\end{tabular} & 10.1 & 79.7 & $<1.00$ & 46.7 & 19.200 & 1.000 & 9.870 & 10.100 & 79.700 & 1.000 & 46.700 \\
\hline
\end{tabular}


Table B-2. PCT Leachate pH Values

\begin{tabular}{|c|c|c|c|c|c|c|c|}
\hline \multicolumn{4}{|c|}{ Set 1} & \multicolumn{4}{|c|}{ Set 2} \\
\hline Identifier & pH & Identifier & pH & Identifier & pH & Identifier & pH \\
\hline ARM-1-1 & 10.12 & EWG-OL-1580-сCC-3 & 10.32 & ARM-1-1 & 10.18 & EWG-OL-3872-ccc-3 & 12.49 \\
\hline ARM-1-2 & 10.15 & EWG-OL-1580-Q-1 & 10.29 & ARM-1-2 & 10.18 & EWG-OL-3872-Q-1 & 11.89 \\
\hline ARM-1-3 & 10.11 & EWG-OL-1580-Q-2 & 10.33 & ARM-1-3 & 10.20 & EWG-OL-3872-Q-2 & 11.85 \\
\hline blank-1 & 6.92 & EWG-OL-1580-Q-3 & 10.31 & blank-1 & 6.97 & EWG-OL-3872-Q-3 & 11.80 \\
\hline blank-2 & 6.93 & EWG-OL-15968-ссс-1 & 11.31 & blank-2 & 6.95 & EWG-OL-5155-сcс-1 & 9.53 \\
\hline EA-1 & 11.55 & EWG-OL-15968-ссс-2 & 11.36 & EA-1 & 11.77 & EWG-OL-5155-ссс-2 & 9.45 \\
\hline EA-2 & 11.54 & EWG-OL-15968-сcс-3 & 11.36 & EA-2 & 11.75 & EWG-OL-5155-сcc-3 & 9.44 \\
\hline EA-3 & 11.55 & EWG-OL-15968-Q-1 & 11.75 & EA-3 & 11.77 & EWG-OL-5155-Q-1 & 9.67 \\
\hline EWG-HAI-Centroid-1-ccc-1 & 9.43 & EWG-OL-15968-Q-2 & 11.69 & EWG-OL-29285-ccc-1 & 8.63 & EWG-OL-5155-Q-2 & 9.65 \\
\hline EWG-HAI-Centroid-1-ccc-2 & 9.43 & EWG-OL-15968-Q-3 & 11.67 & EWG-OL-29285-сcс-2 & 8.66 & EWG-OL-5155-Q-3 & 9.58 \\
\hline EWG-HAI-Centroid-1-ccc-3 & 9.44 & EWG-OL-1672-сcс-1 & 8.58 & EWG-OL-29285-сcс-3 & 8.71 & EWG-OL-5801-сcс-1 & 9.61 \\
\hline EWG-HAI-Centroid-1-Q-1 & 9.80 & EWG-OL-1672-сcс-2 & 8.59 & EWG-OL-29285-Q-1 & 8.92 & EWG-OL-5801-cсc-2 & 9.49 \\
\hline EWG-HAI-Centroid-1-Q-2 & 9.79 & EWG-OL-1672-ссс-3 & 8.46 & EWG-OL-29285-Q-2 & 8.83 & EWG-OL-5801-ссс-3 & 9.49 \\
\hline EWG-HAI-Centroid-1-Q-3 & 9.76 & EWG-OL-1672-Q-1 & 8.83 & EWG-OL-29285-Q-3 & 8.92 & EWG-OL-5801-Q-1 & 9.51 \\
\hline EWG-HAI-Centroid-2-сcс-1 & 9.44 & EWG-OL-1672-Q-2 & 8.78 & EWG-OL-31644-сcс-1 & 9.29 & EWG-OL-5801-Q-2 & 9.52 \\
\hline EWG-HAI-Centroid-2-ccc-2 & 9.39 & EWG-OL-1672-Q-3 & 8.71 & EWG-OL-31644-сcс-2 & 9.28 & EWG-OL-5801-Q-3 & 9.45 \\
\hline EWG-HAI-Centroid-2-ccc-3 & 9.25 & EWG-OL-2463-ccc-1 & 12.65 & EWG-OL-31644-ccc-3 & 9.31 & EWG-OL-6198-сcс-1 & 9.45 \\
\hline EWG-HAI-Centroid-2-Q-1 & 9.30 & EWG-OL-2463-сcс-2 & 12.70 & EWG-OL-31644-Q-1 & 9.84 & EWG-OL-6198-сcс-2 & 9.38 \\
\hline EWG-HAI-Centroid-2-Q-2 & 9.24 & EWG-OL-2463-ссс-3 & 12.70 & EWG-OL-31644-Q-2 & 9.82 & EWG-OL-6198-ссс-3 & 9.42 \\
\hline EWG-HAI-Centroid-2-Q-3 & 9.28 & EWG-OL-2463-Q-1 & 12.15 & EWG-OL-31644-Q-3 & 9.81 & EWG-OL-6198-Q-1 & 9.68 \\
\hline EWG-OL-1369-ccc-1 & 10.24 & EWG-OL-2463-Q-2 & 12.21 & EWG-OL-33115-ccc-1 & 8.80 & EWG-OL-6198-Q-2 & 9.58 \\
\hline EWG-OL-1369-сcс-2 & 10.22 & EWG-OL-2463-Q-3 & 12.21 & EWG-OL-33115-сcc-2 & 8.85 & EWG-OL-6198-Q-3 & 9.54 \\
\hline EWG-OL-1369-ссс-3 & 10.24 & EWG-OL-26012-ссс-1 & 11.36 & EWG-OL-33115-сcс-3 & 8.93 & EWG-OL-801-сcс-1 & 9.65 \\
\hline EWG-OL-1369-Q-1 & 10.41 & EWG-OL-26012-ссс-2 & 11.38 & EWG-OL-33115-Q-1 & 9.70 & EWG-OL-801-сcс-2 & 9.63 \\
\hline EWG-OL-1369-Q-2 & 10.41 & EWG-OL-26012-сcс-3 & 11.38 & EWG-OL-33115-Q-2 & 9.65 & EWG-OL-801-cCC-3 & 9.63 \\
\hline EWG-OL-1369-Q-3 & 10.43 & EWG-OL-26012-Q-1 & 10.51 & EWG-OL-33115-Q-3 & 9.72 & EWG-OL-801-Q-1 & 9.79 \\
\hline EWG-OL-14827-cCc-1 & 10.20 & EWG-OL-26012-Q-2 & 10.54 & EWG-OL-38552-ccc-1 & 9.34 & EWG-OL-801-Q-2 & 9.68 \\
\hline EWG-OL-14827-сcс-2 & 10.26 & EWG-OL-26012-Q-3 & 10.61 & EWG-OL-38552-сcс-2 & 9.38 & EWG-OL-801-Q-3 & 9.69 \\
\hline EWG-OL-14827-ссс-3 & 10.26 & EWG-OL-2619-ссс-1 & 9.09 & EWG-OL-38552-сcс-3 & 9.32 & RSM-EWG-1-034-ссс-1 & 9.31 \\
\hline EWG-OL-14827-Q-1 & 10.14 & EWG-OL-2619-сcс-2 & 9.00 & EWG-OL-38552-Q-1 & 9.50 & RSM-EWG-1-034-сcс-2 & 9.31 \\
\hline EWG-OL-14827-Q-2 & 10.17 & EWG-OL-2619-ссс-3 & 8.98 & EWG-OL-38552-Q-2 & 9.58 & RSM-EWG-1-034-сcс-3 & 9.22 \\
\hline EWG-OL-14827-Q-3 & 10.19 & EWG-OL-2619-Q-1 & 9.07 & EWG-OL-38552-Q-3 & 9.68 & RSM-EWG-1-034-Q-1 & 9.61 \\
\hline EWG-OL-1580-ссс-1 & 10.32 & EWG-OL-2619-Q-2 & 9.06 & EWG-OL-3872-ссс-1 & 12.48 & RSM-EWG-1-034-Q-2 & 9.38 \\
\hline EWG-OL-1580-сcс-2 & 10.30 & EWG-OL-2619-Q-3 & 9.07 & EWG-OL-3872-ссс-2 & 12.49 & RSM-EWG-1-034-Q-3 & 9.40 \\
\hline
\end{tabular}


Exhibit B-1. PCT Measurements in Analytical Sequence by Analytical Set

Analytical Set $=1$

Variability Chart for $\log [B$ ppm]

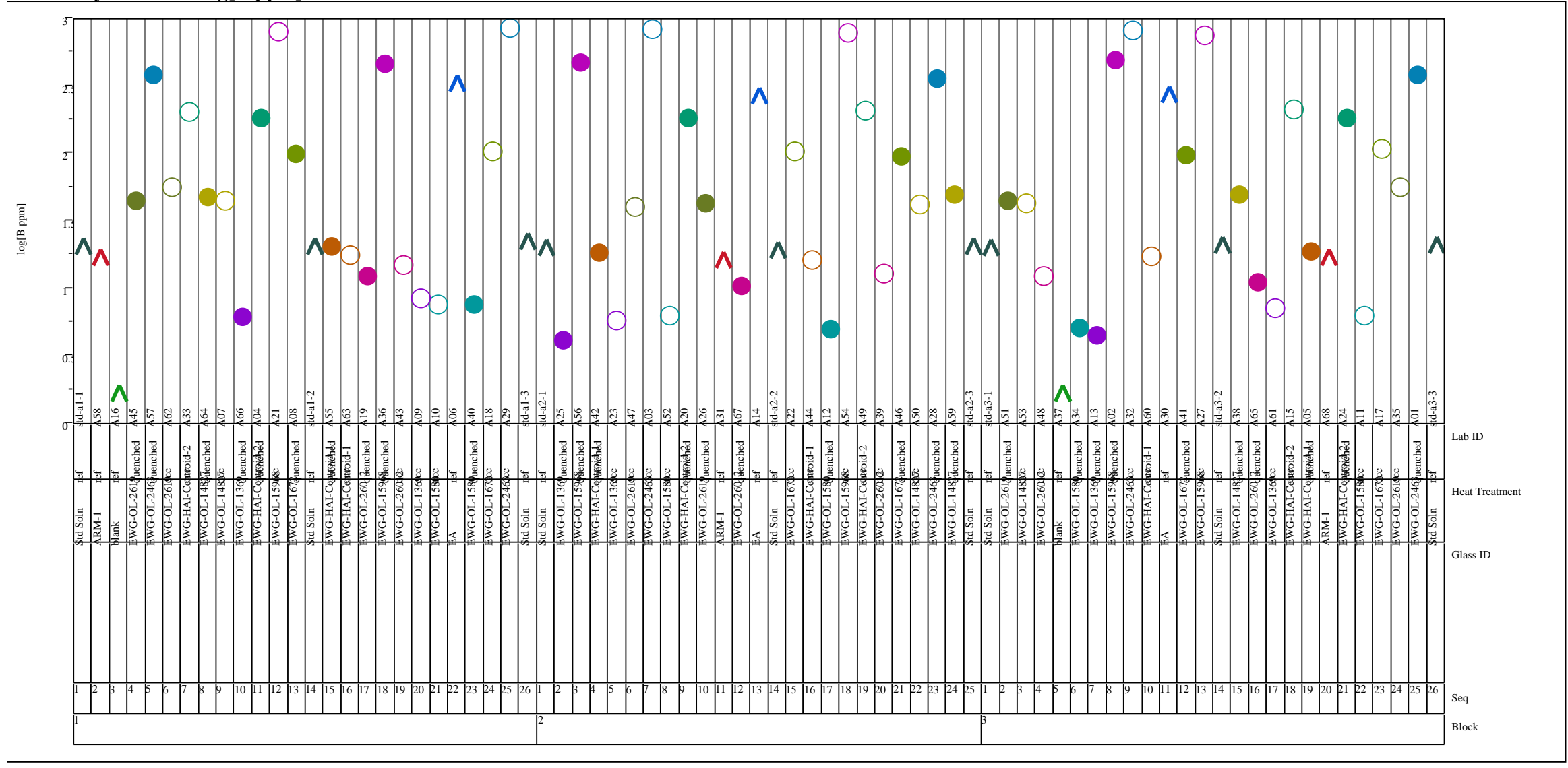




\section{Exhibit B-1. PCT Measurements in Analytical Sequence by Analytical Set (continued)}

Analytical Set $=1$

Variability Chart for $\log [\mathrm{Ca}$ ppm $]$

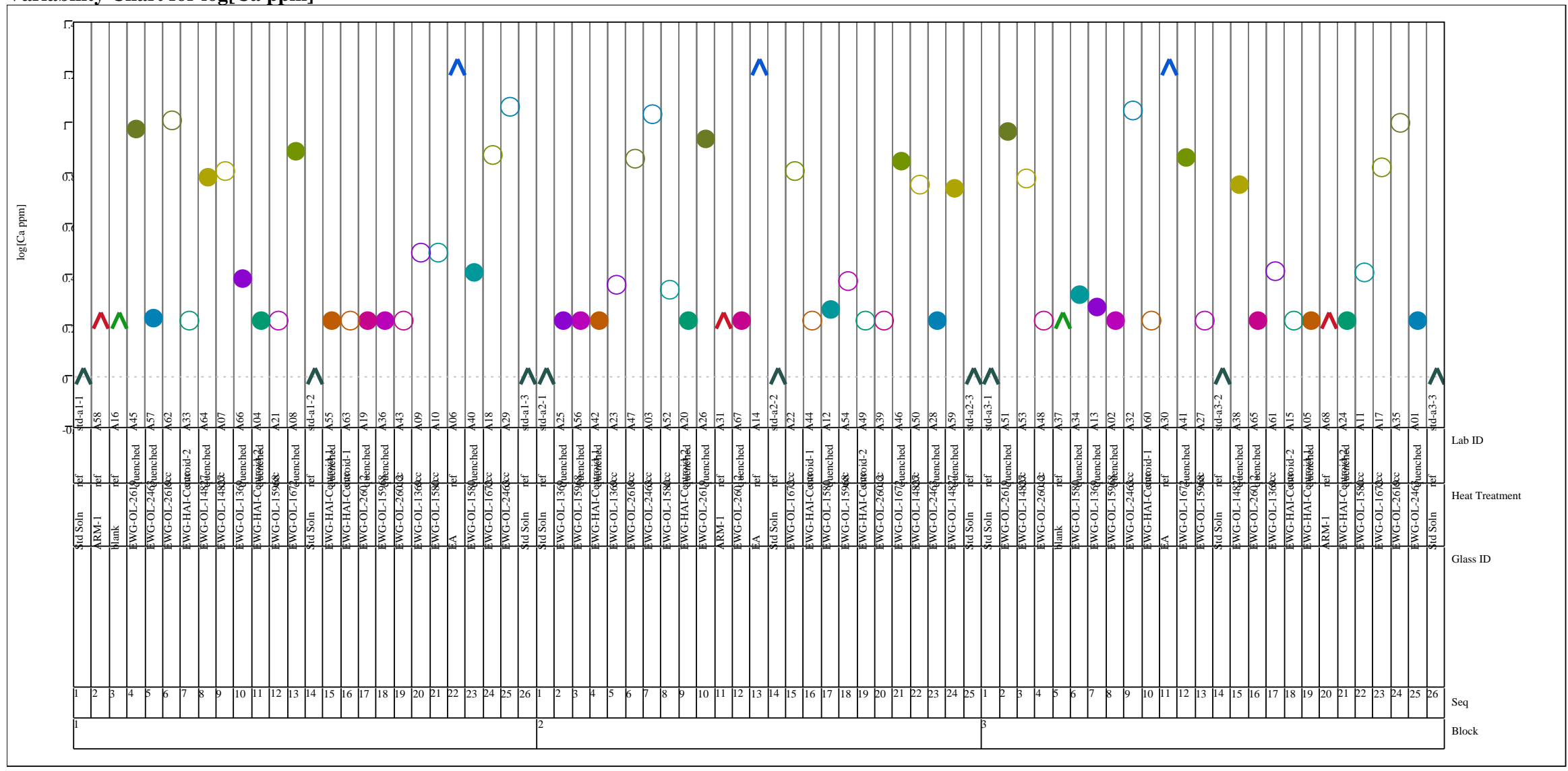




\section{Exhibit B-1. PCT Measurements in Analytical Sequence by Analytical Set (continued)}

Analytical Set $=1$

Variability Chart for $\log [\mathrm{K}$ ppm]

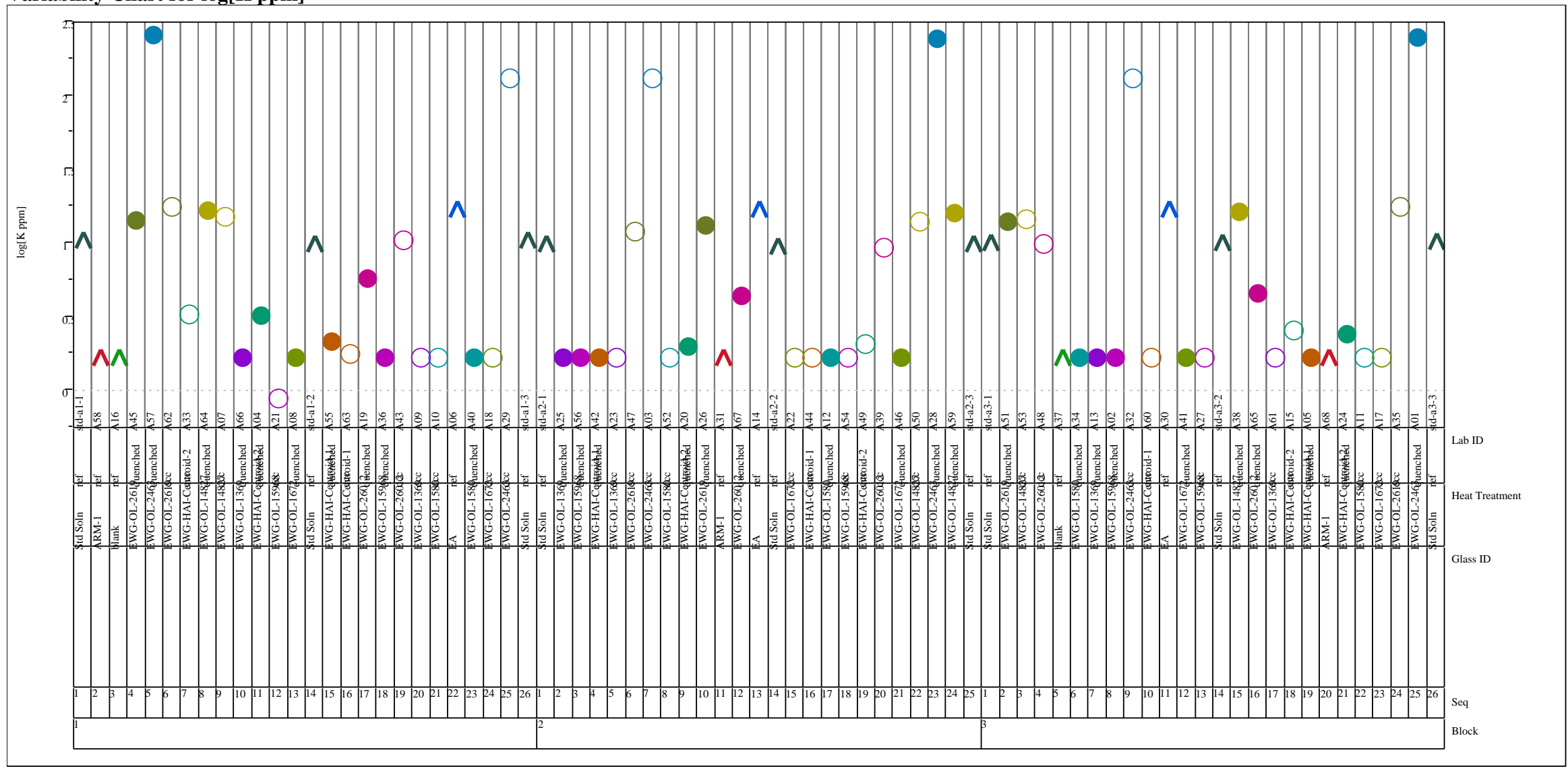




\section{Exhibit B-1. PCT Measurements in Analytical Sequence by Analytical Set (continued)}

Analytical Set $=1$

Variability Chart for $\log [\mathrm{Li}$ ppm]

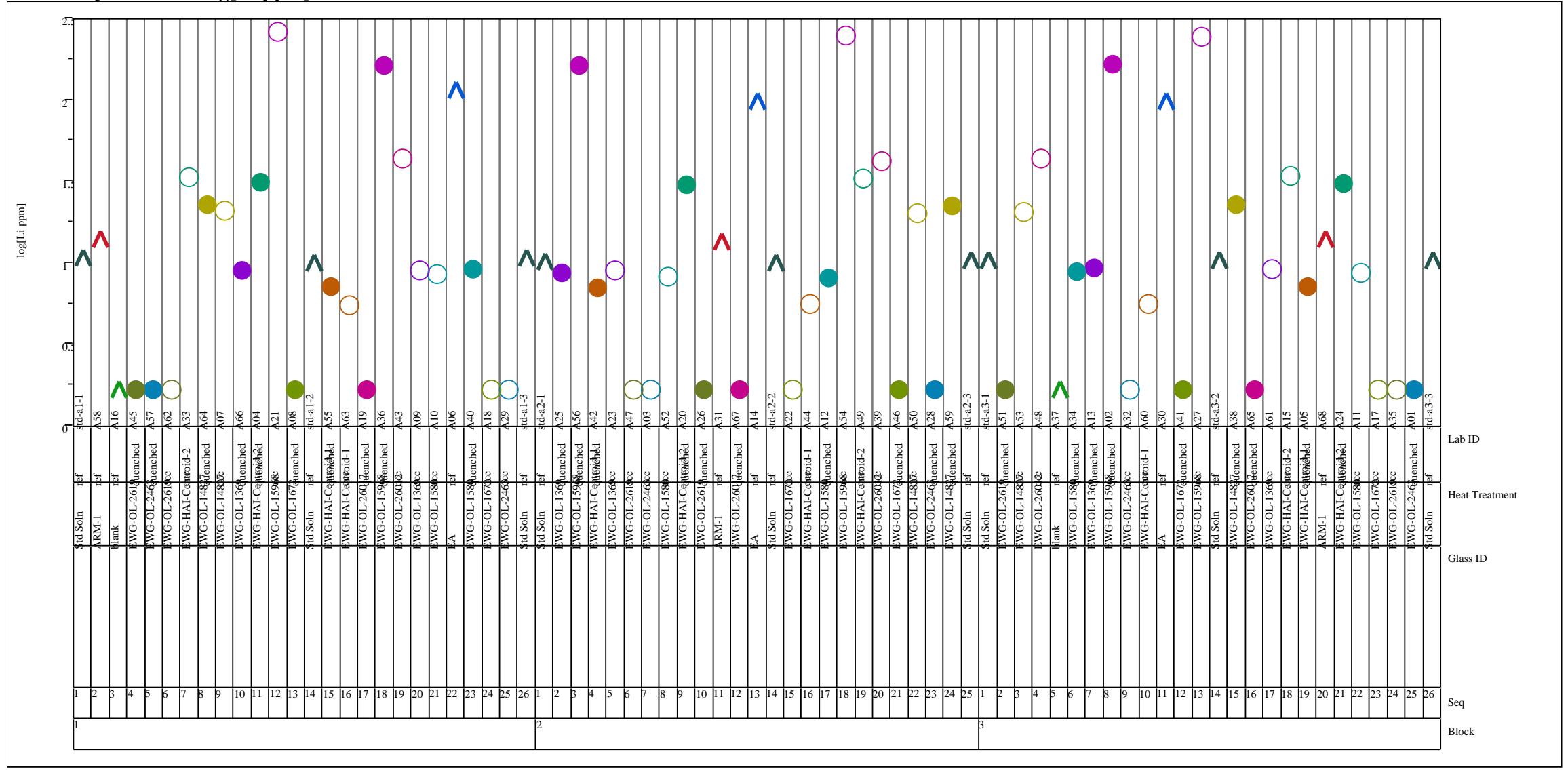




\section{Exhibit B-1. PCT Measurements in Analytical Sequence by Analytical Set (continued)}

Analytical Set $=1$

Variability Chart for $\log [\mathrm{Na}$ ppm]

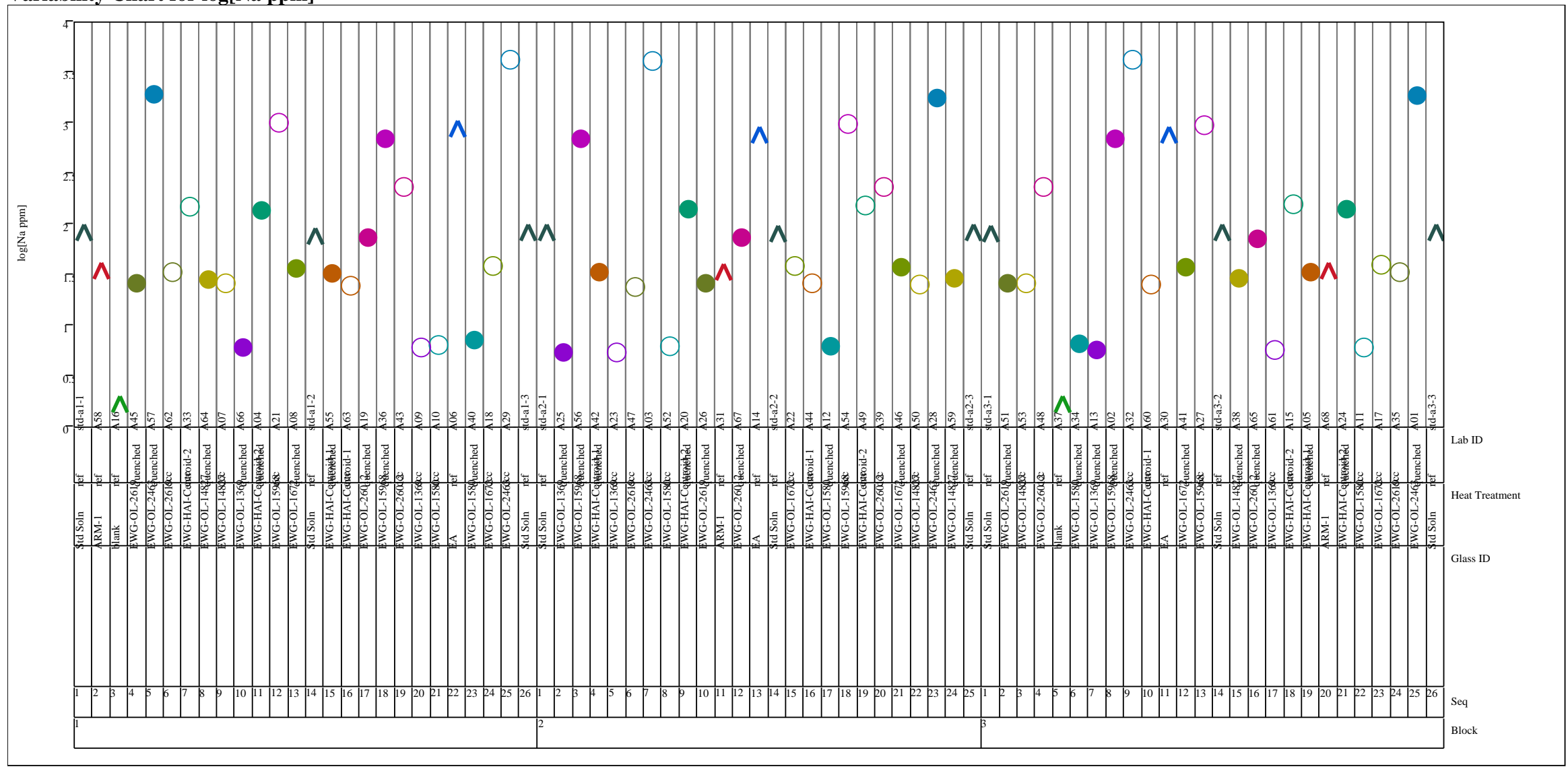




\section{Exhibit B-1. PCT Measurements in Analytical Sequence by Analytical Set (continued)}

Analytical Set $=1$

Variability Chart for $\log [\mathrm{P}$ ppm $]$

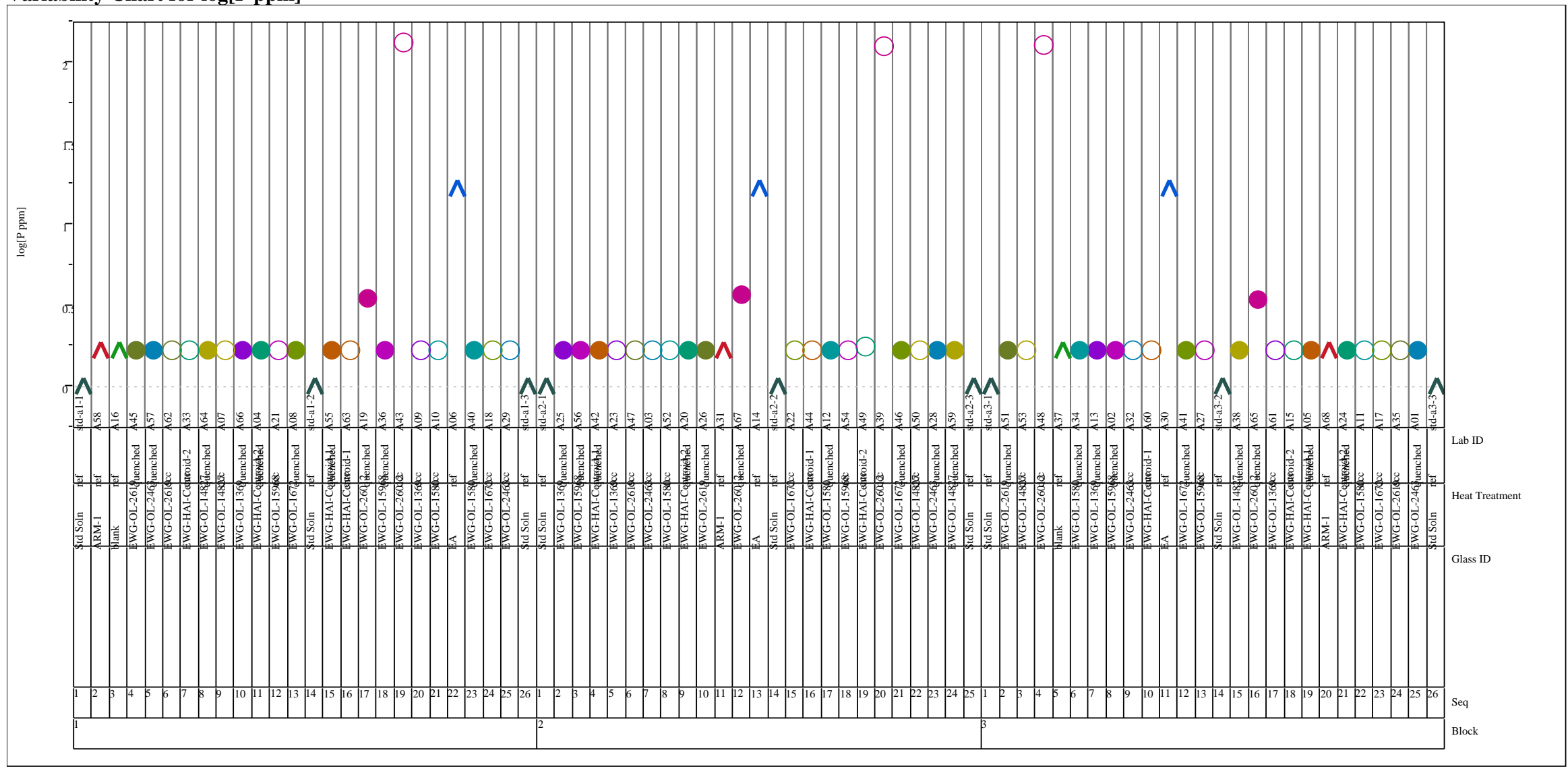




\section{Exhibit B-1. PCT Measurements in Analytical Sequence by Analytical Set (continued)}

Analytical Set $=1$

Variability Chart for $\log [\mathrm{Si}$ ppm $]$

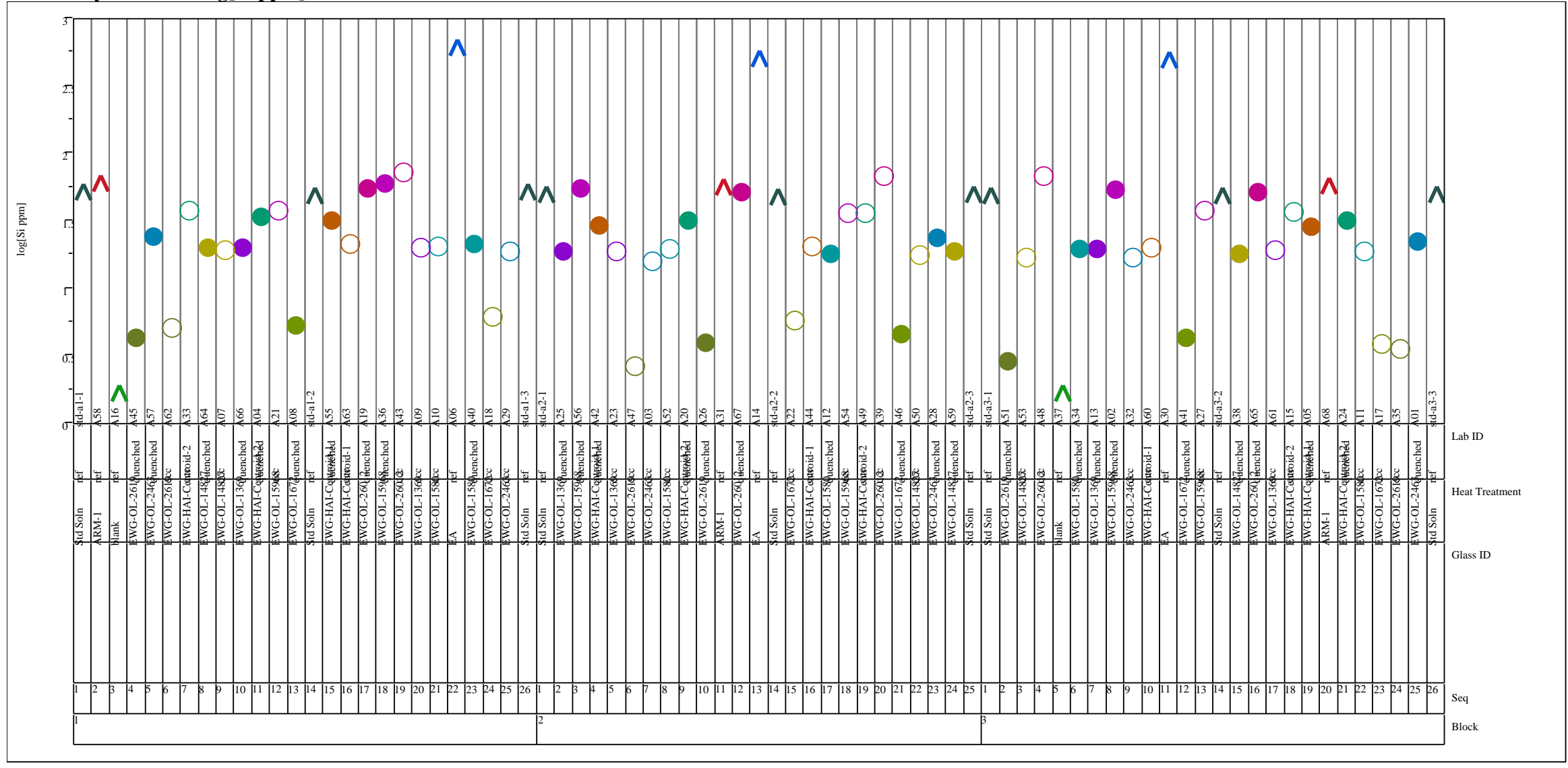




\section{Exhibit B-1. PCT Measurements in Analytical Sequence by Analytical Set (continued)}

Analytical Set $=2$

Variability Chart for $\log [\mathrm{B}$ ppm]

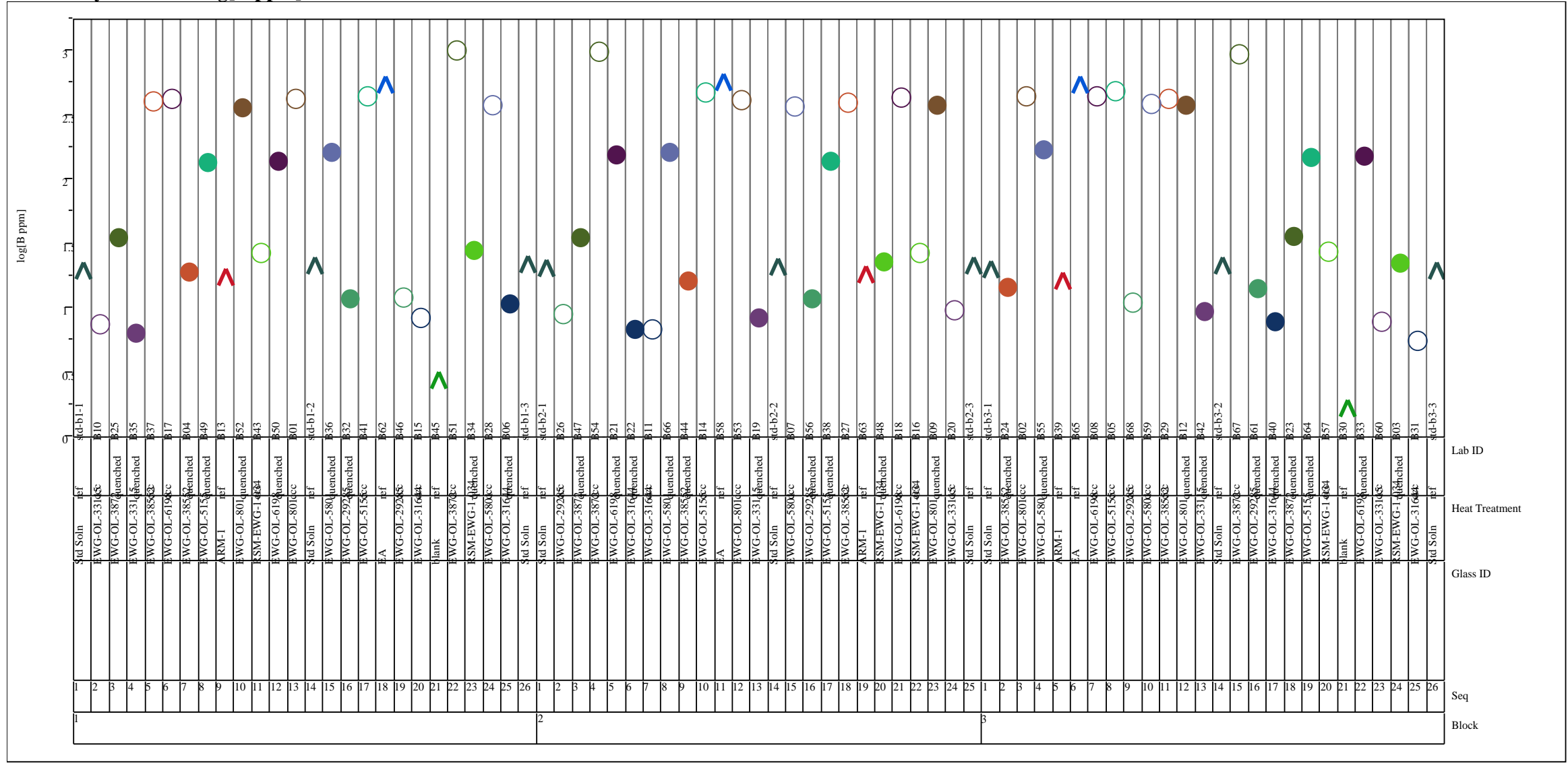




\section{Exhibit B-1. PCT Measurements in Analytical Sequence by Analytical Set (continued)}

Analytical Set $=2$

Variability Chart for $\log [\mathrm{Ca}$ ppm]

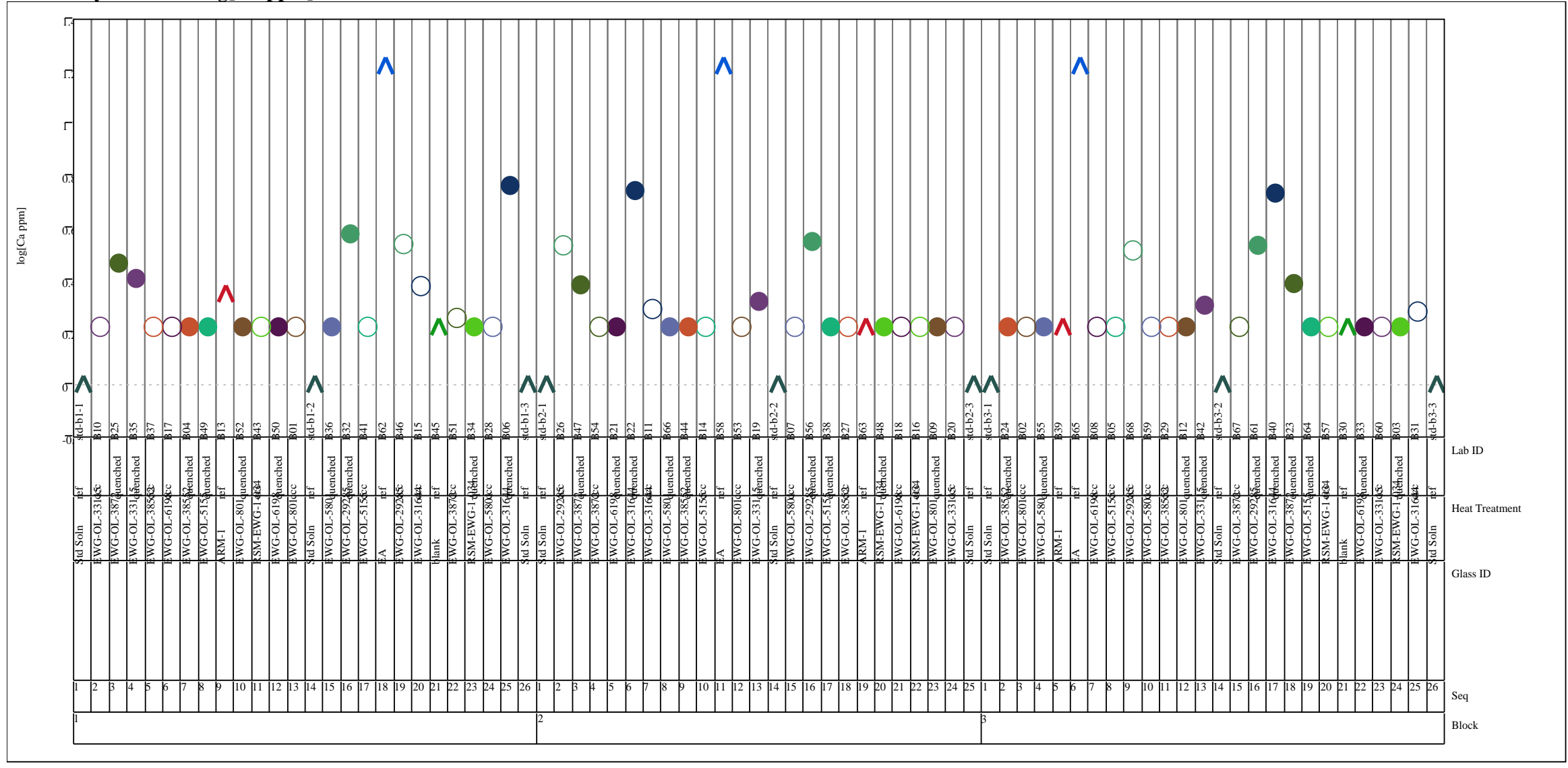




\section{Exhibit B-1. PCT Measurements in Analytical Sequence by Analytical Set (continued)}

Analytical Set $=2$

Variability Chart for $\log [\mathrm{K}$ ppm]

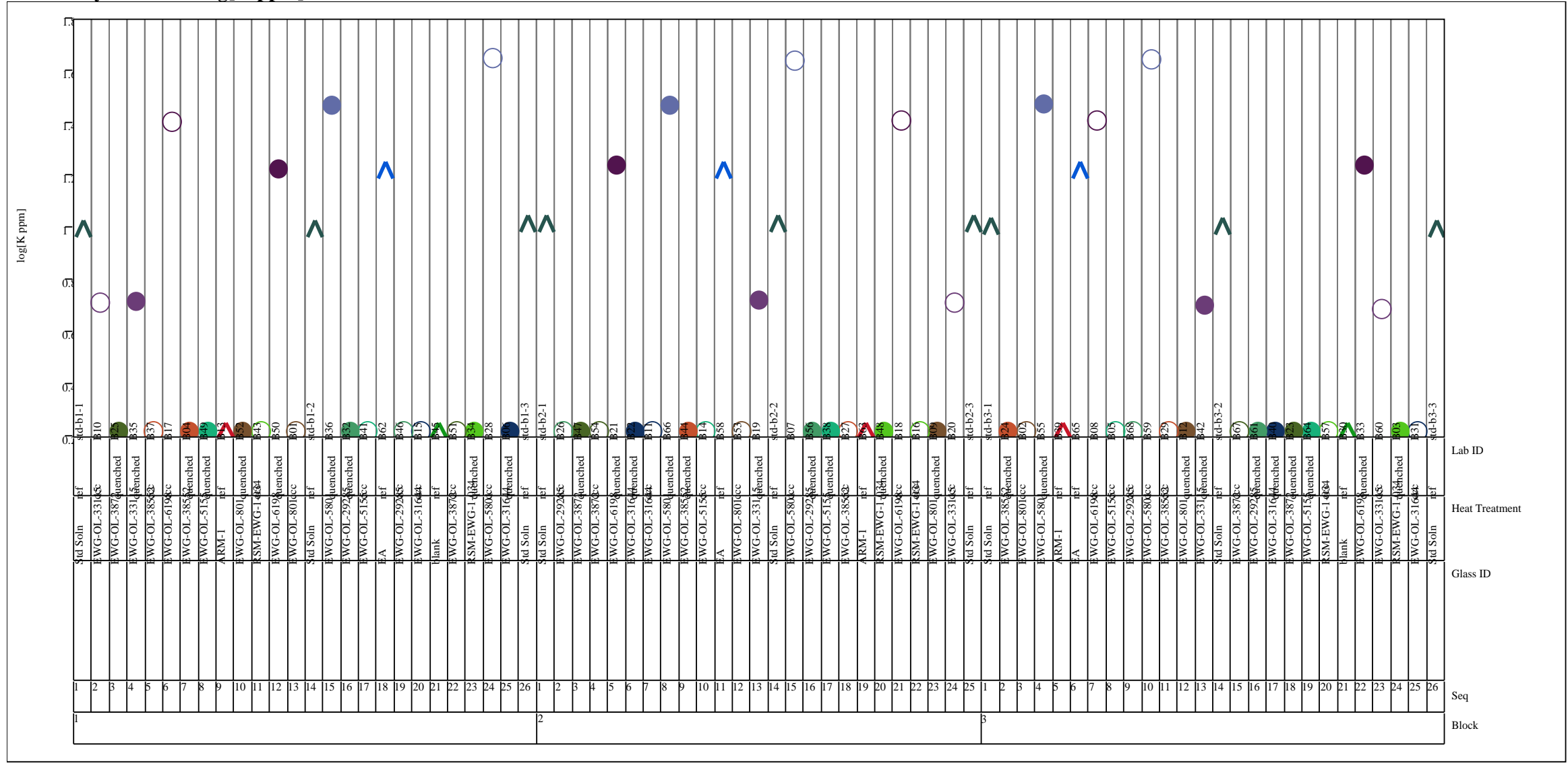




\section{Exhibit B-1. PCT Measurements in Analytical Sequence by Analytical Set (continued)}

Analytical Set $=2$

Variability Chart for $\log [\mathrm{Li}$ ppm]

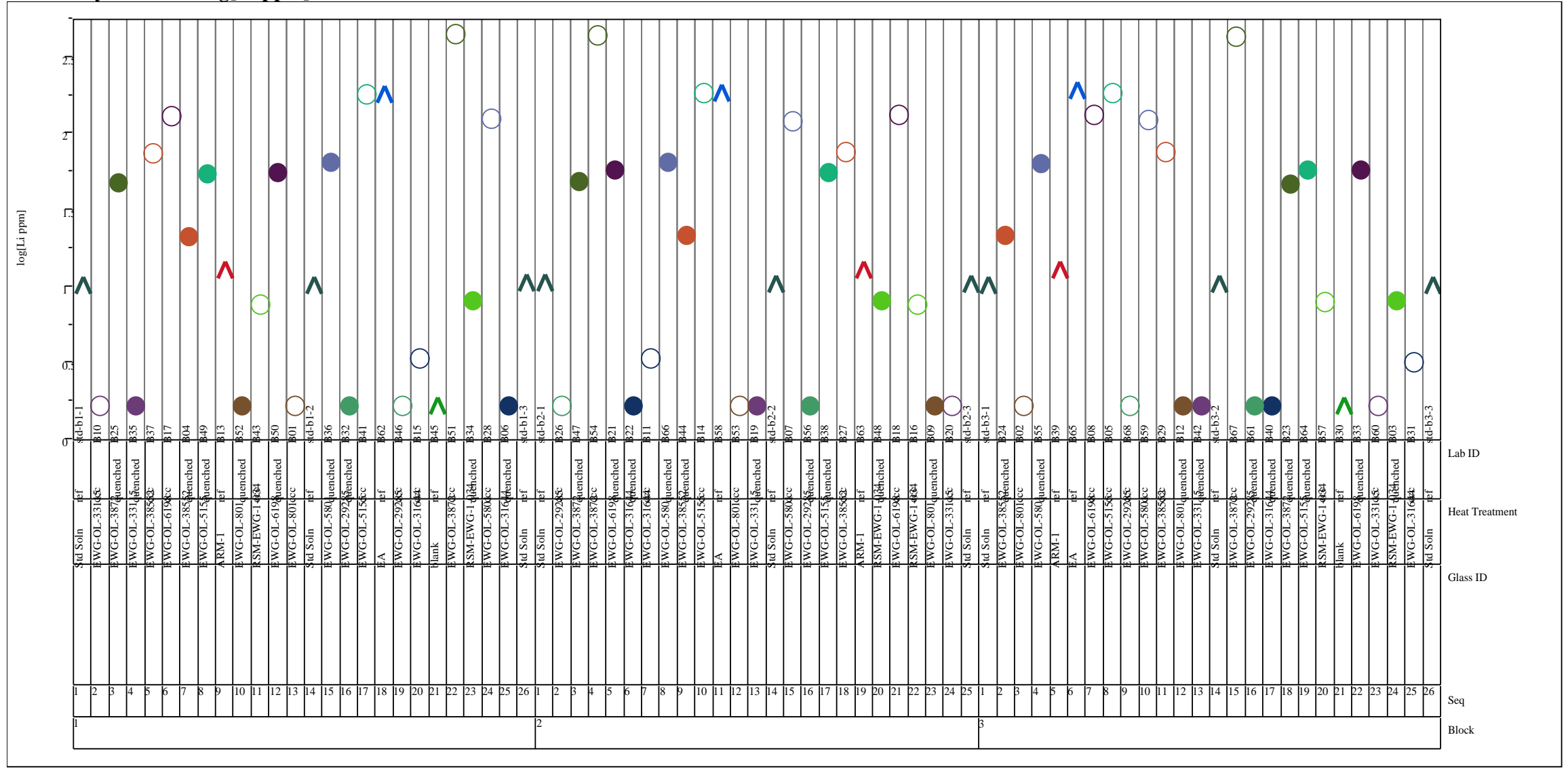




\section{Exhibit B-1. PCT Measurements in Analytical Sequence by Analytical Set (continued)}

Analytical Set $=2$

Variability Chart for $\log [\mathrm{Na}$ ppm $]$

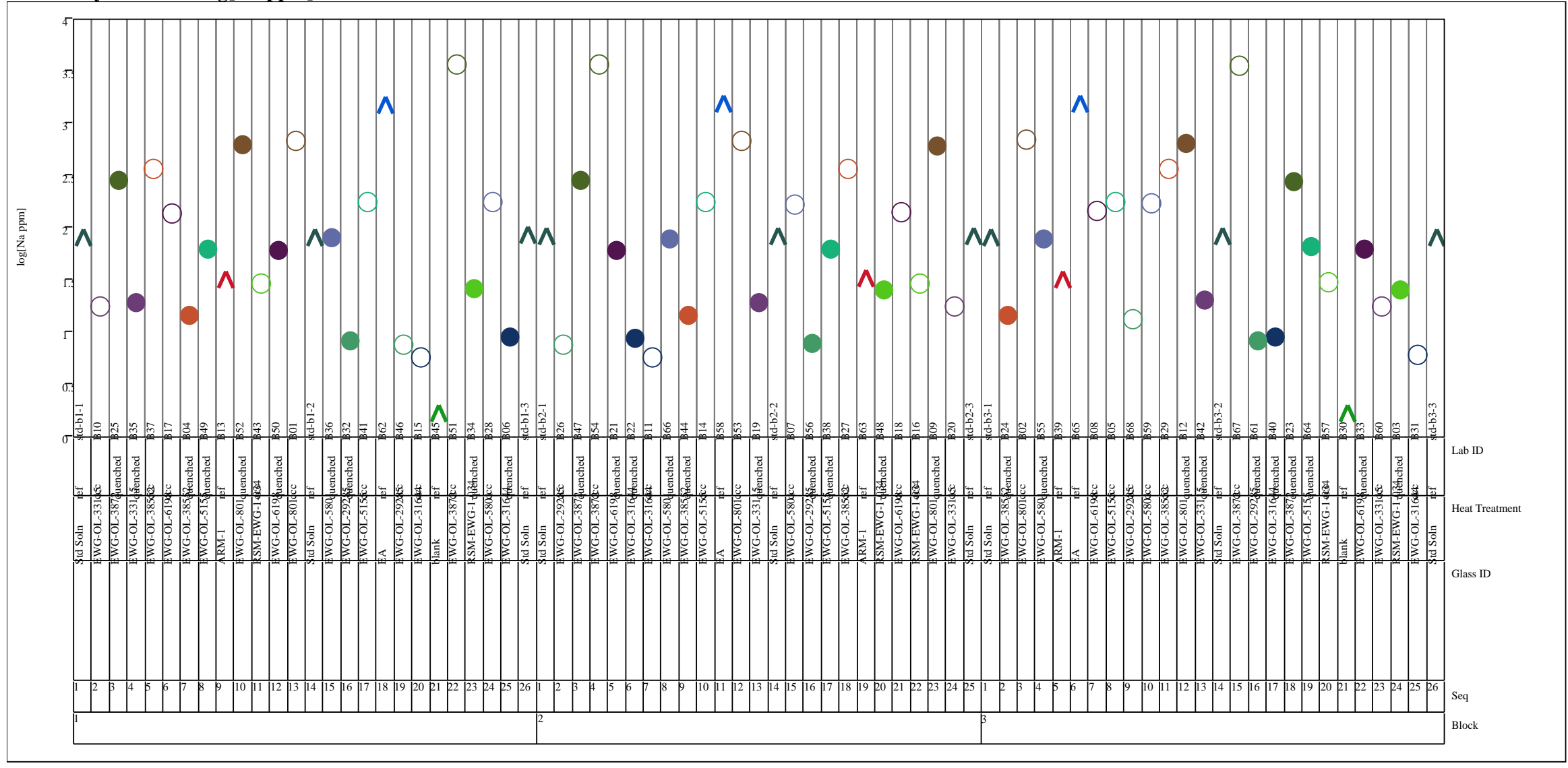




\section{Exhibit B-1. PCT Measurements in Analytical Sequence by Analytical Set (continued)}

Analytical Set $=2$

Variability Chart for $\log [\mathrm{P}$ ppm $]$

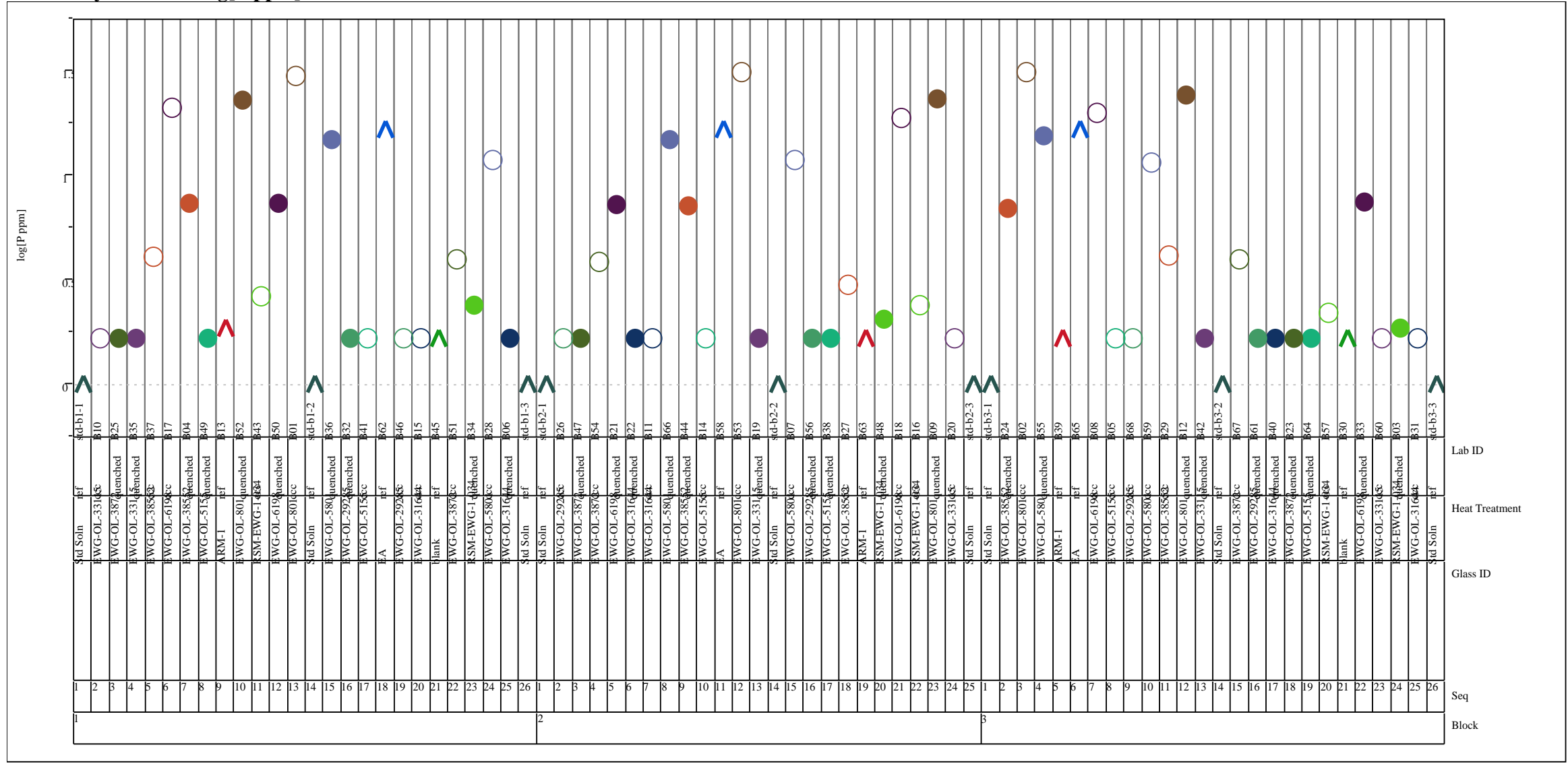




\section{Exhibit B-1. PCT Measurements in Analytical Sequence by Analytical Set (continued)}

Analytical Set $=2$

Variability Chart for $\log [\mathrm{Si}$ ppm]

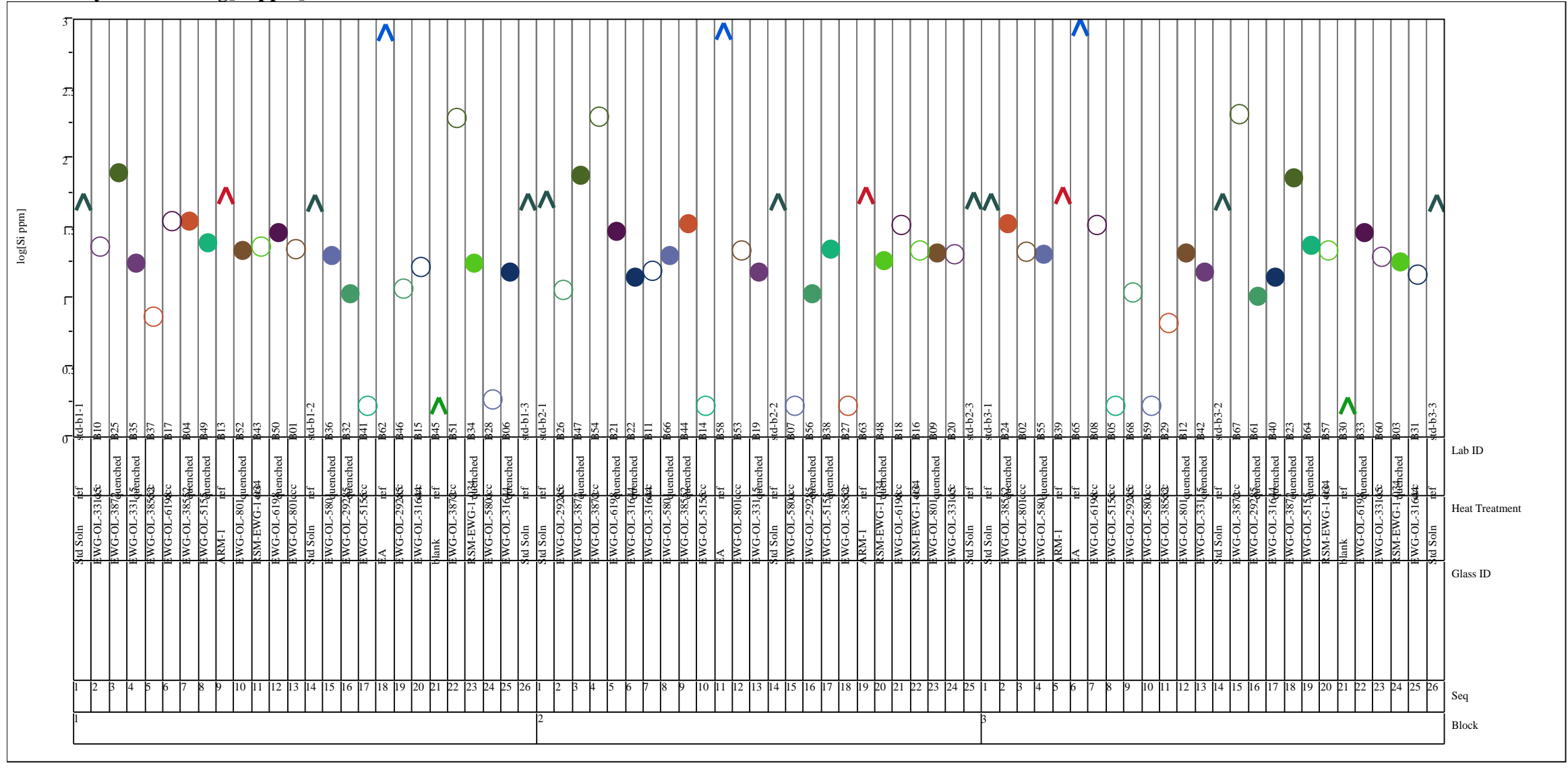


Exhibit B-2. PCT Measurements for Each Glass Grouped by Heat Treatment

Analytical Set $=1$

Variability Chart for log[B ppm]

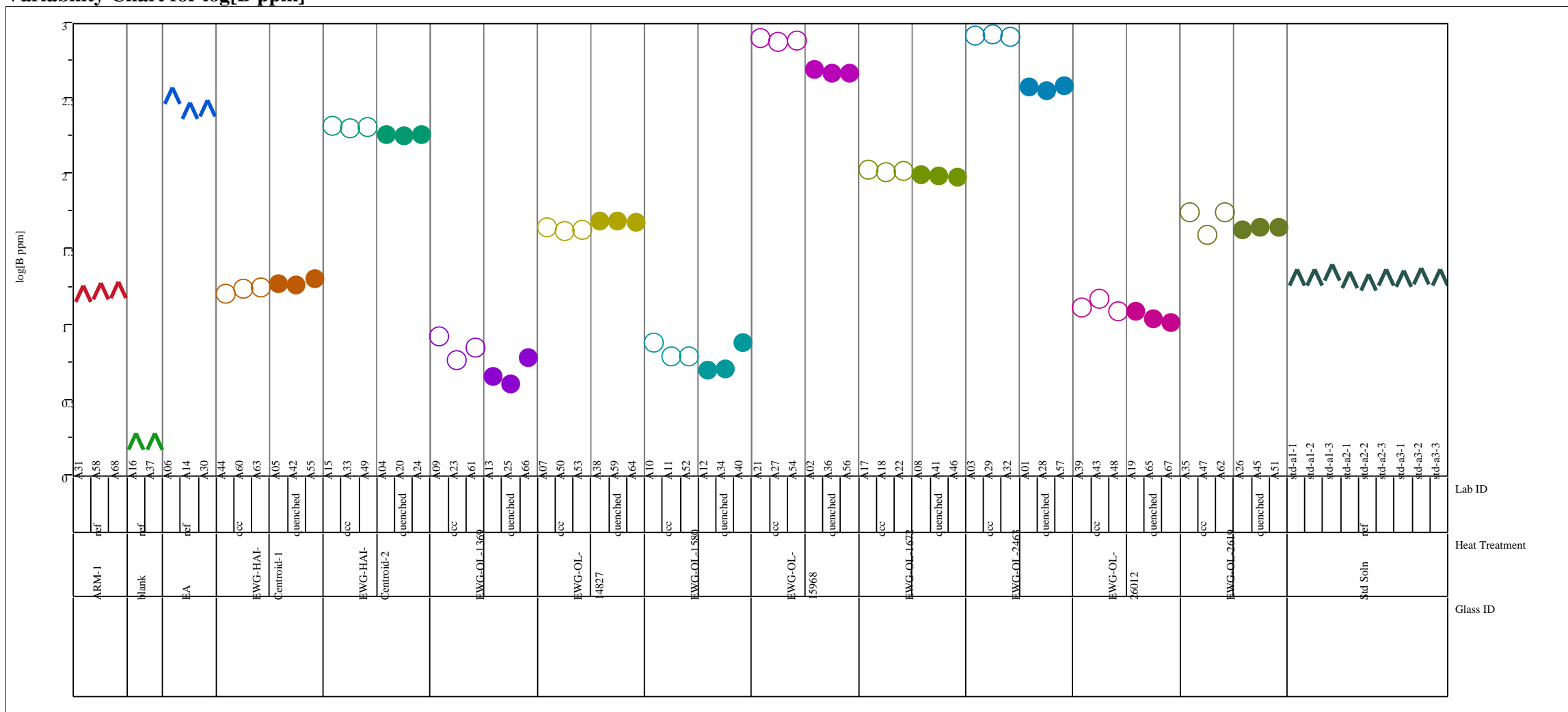




\section{Exhibit B-2. PCT Measurements for Each Glass Grouped by Heat Treatment (continued)}

Analytical Set $=1$

Variability Chart for log[Ca ppm

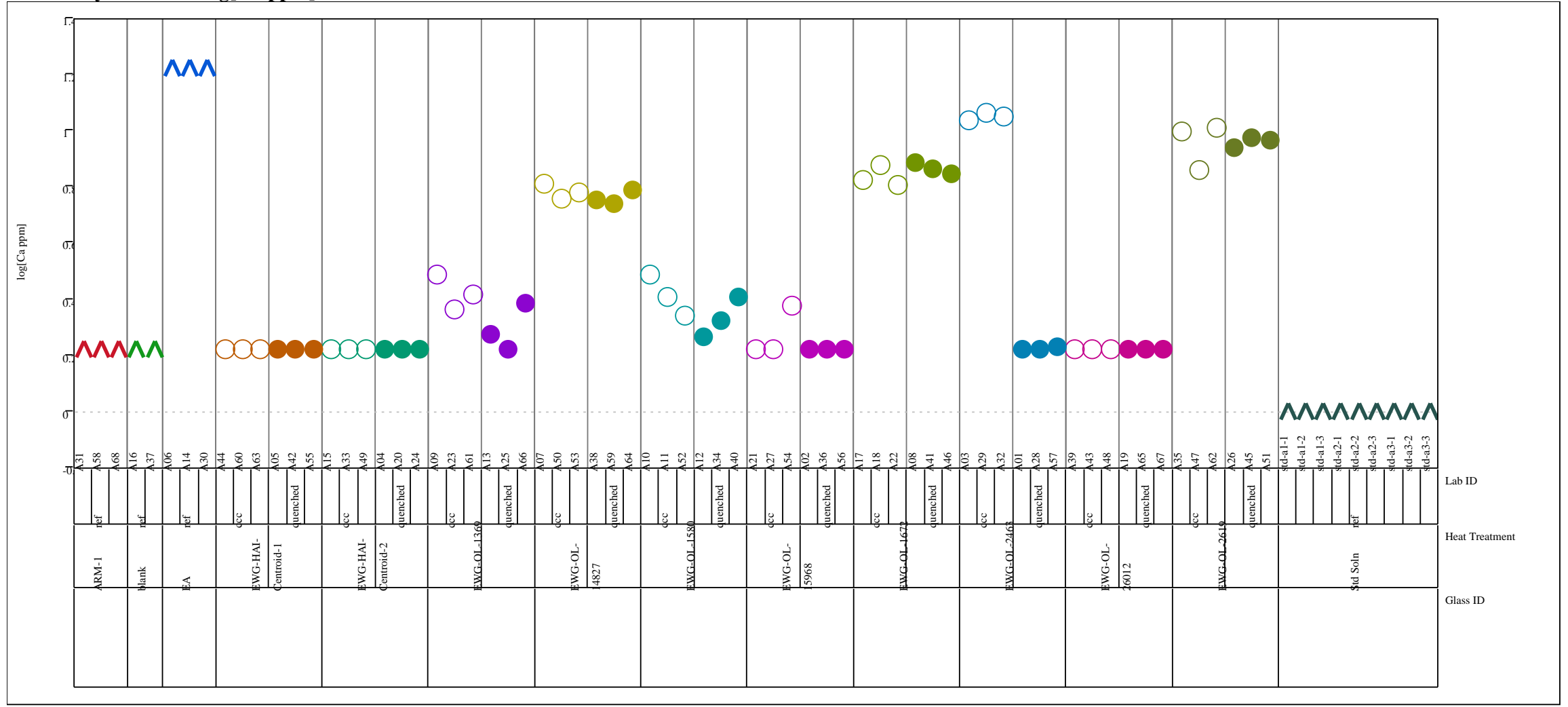




\section{Exhibit B-2. PCT Measurements for Each Glass Grouped by Heat Treatment (continued)}

Analytical Set $=1$

Variability Chart for $\log [\mathrm{K}$ ppm]

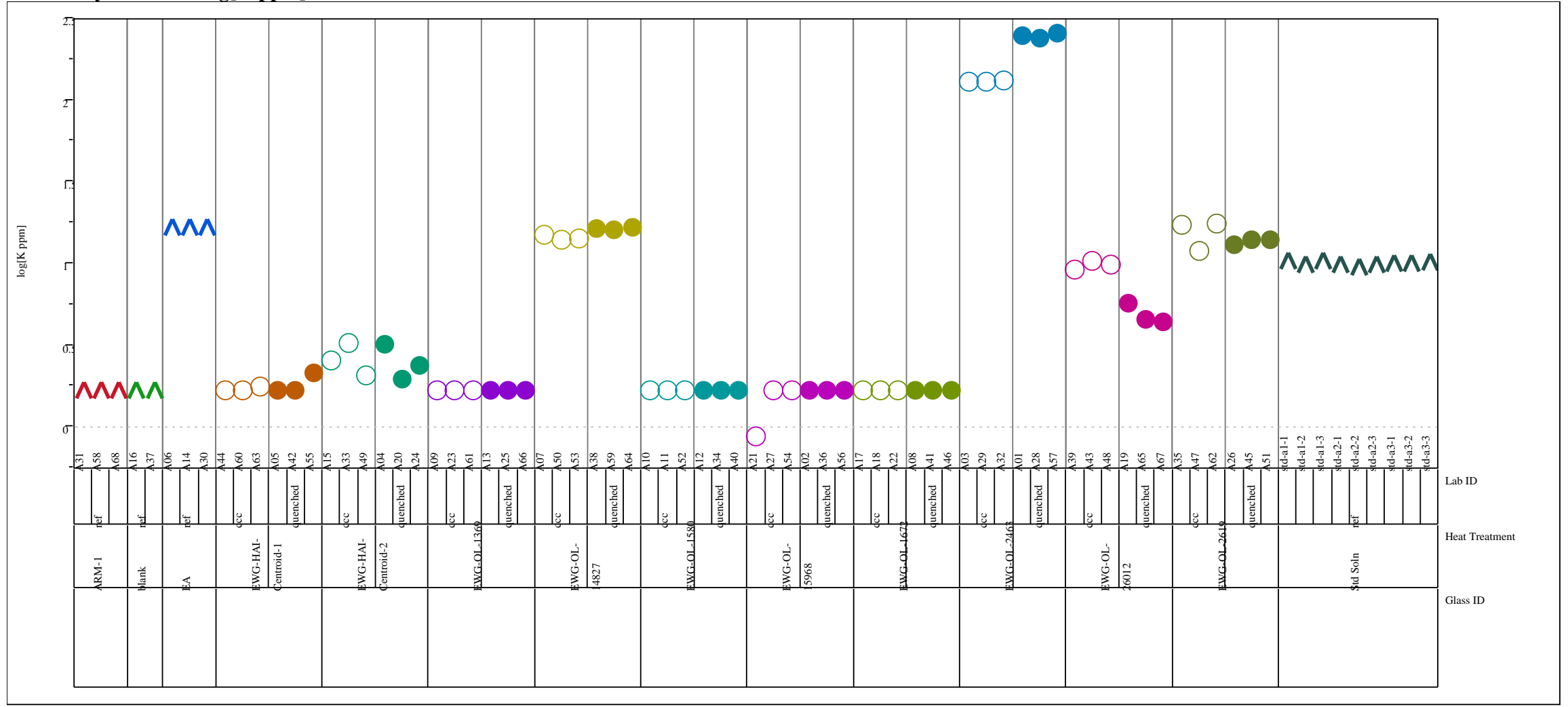




\section{Exhibit B-2. PCT Measurements for Each Glass Grouped by Heat Treatment (continued)}

Analytical Set $=1$

Variability Chart for $\log [\mathrm{Li}$ ppm $]$

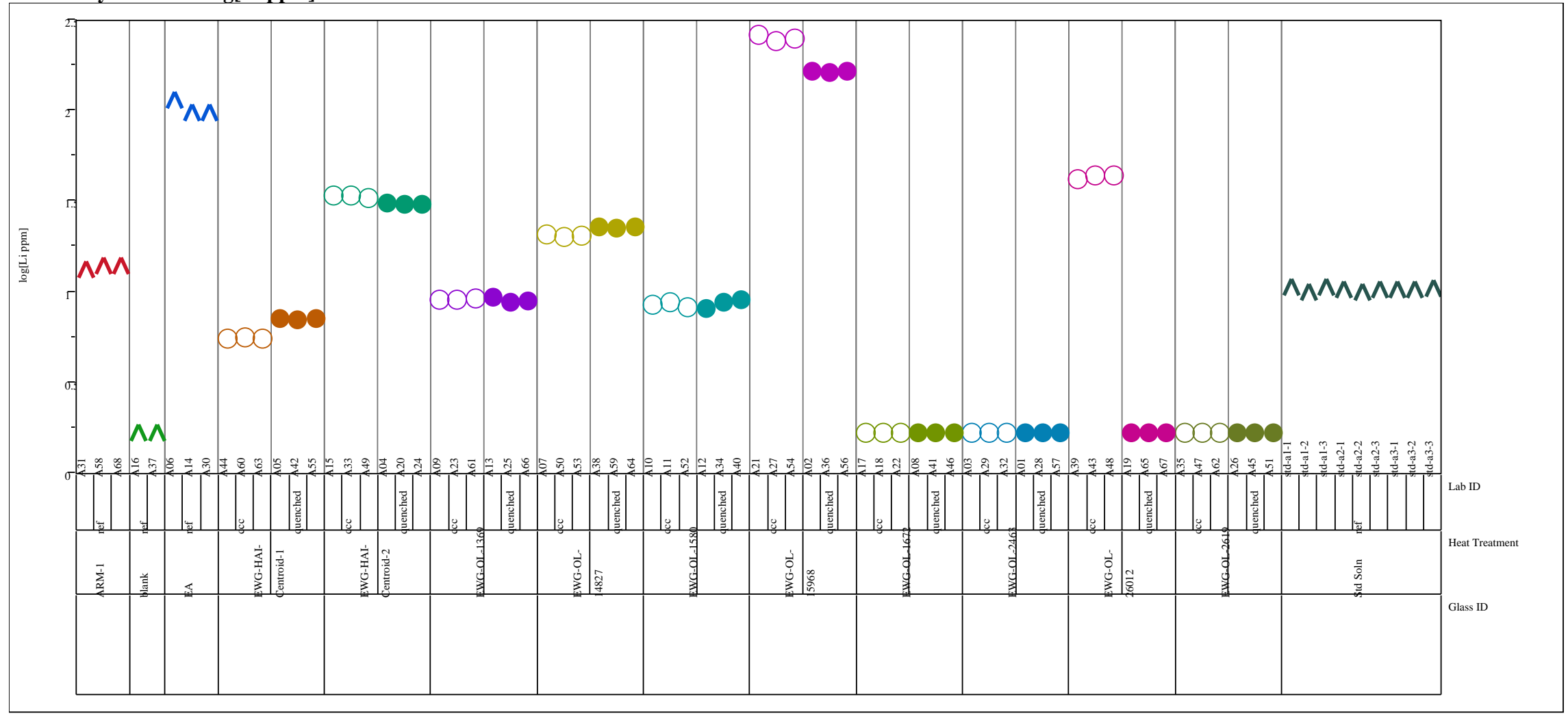




\section{Exhibit B-2. PCT Measurements for Each Glass Grouped by Heat Treatment (continued)}

Analytical Set $=1$

Variability Chart for $\log [\mathrm{Na}$ ppm

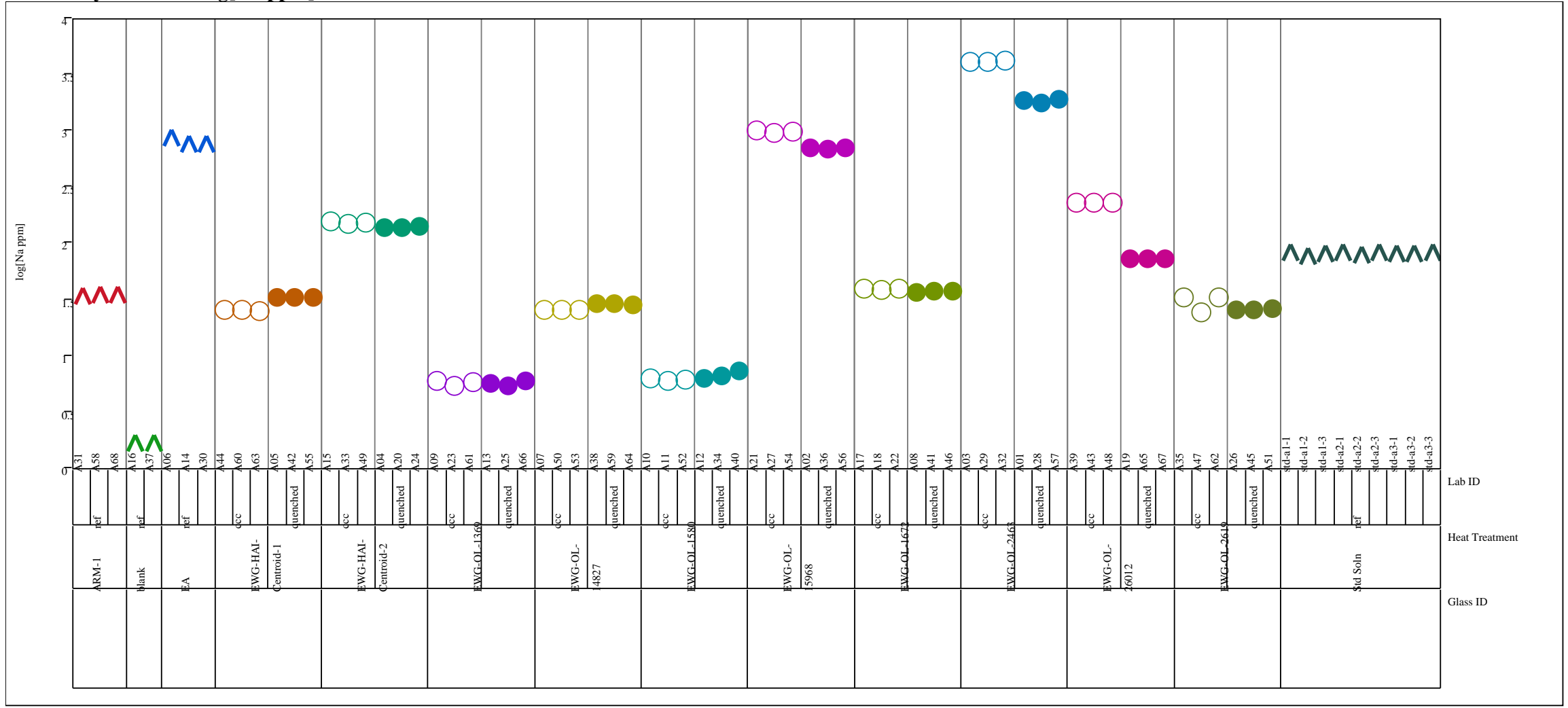




\section{Exhibit B-2. PCT Measurements for Each Glass Grouped by Heat Treatment (continued)}

Analytical Set $=1$

Variability Chart for $\log [\mathbf{P}$ ppm]

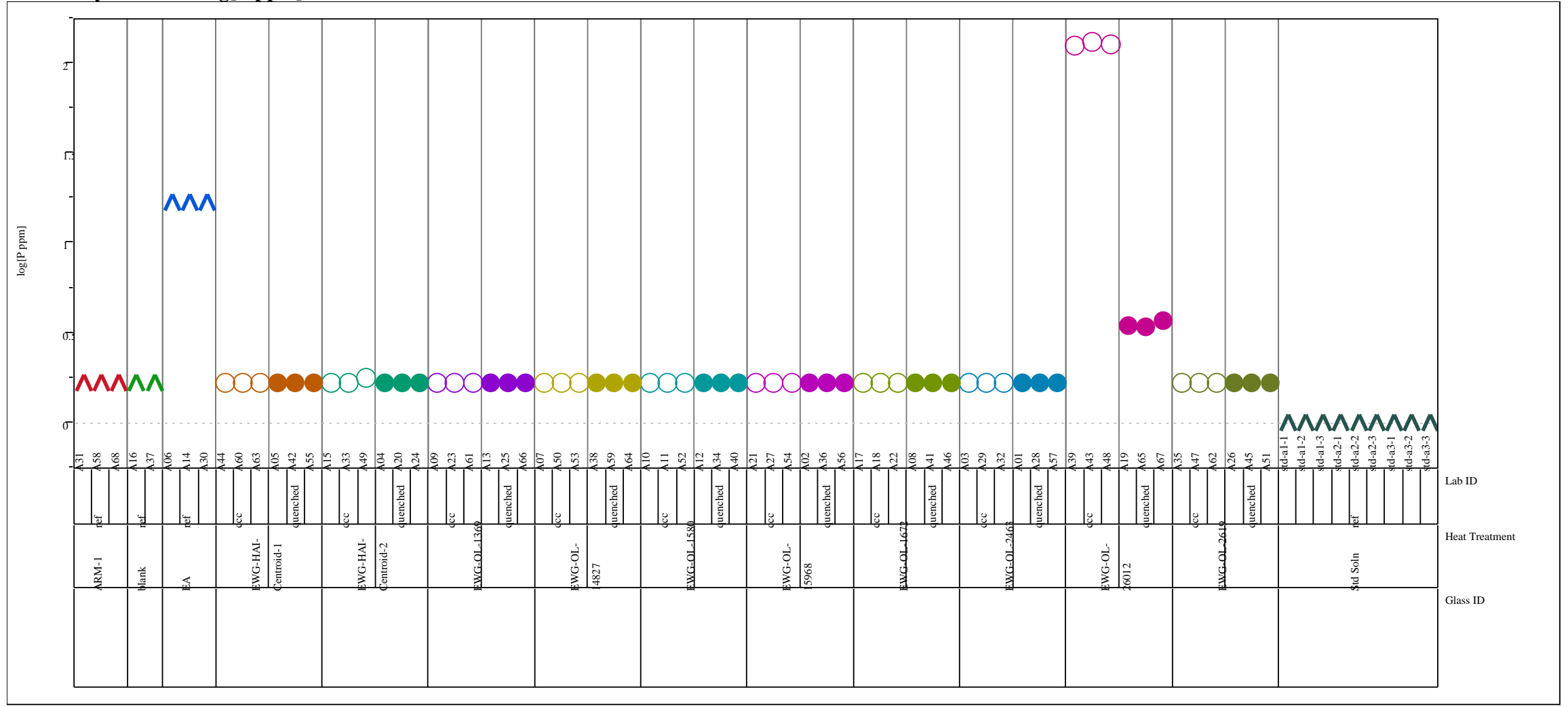




\section{Exhibit B-2. PCT Measurements for Each Glass Grouped by Heat Treatment (continued)}

Analytical Set $=1$

Variability Chart for $\log [\mathrm{Si}$ ppm]

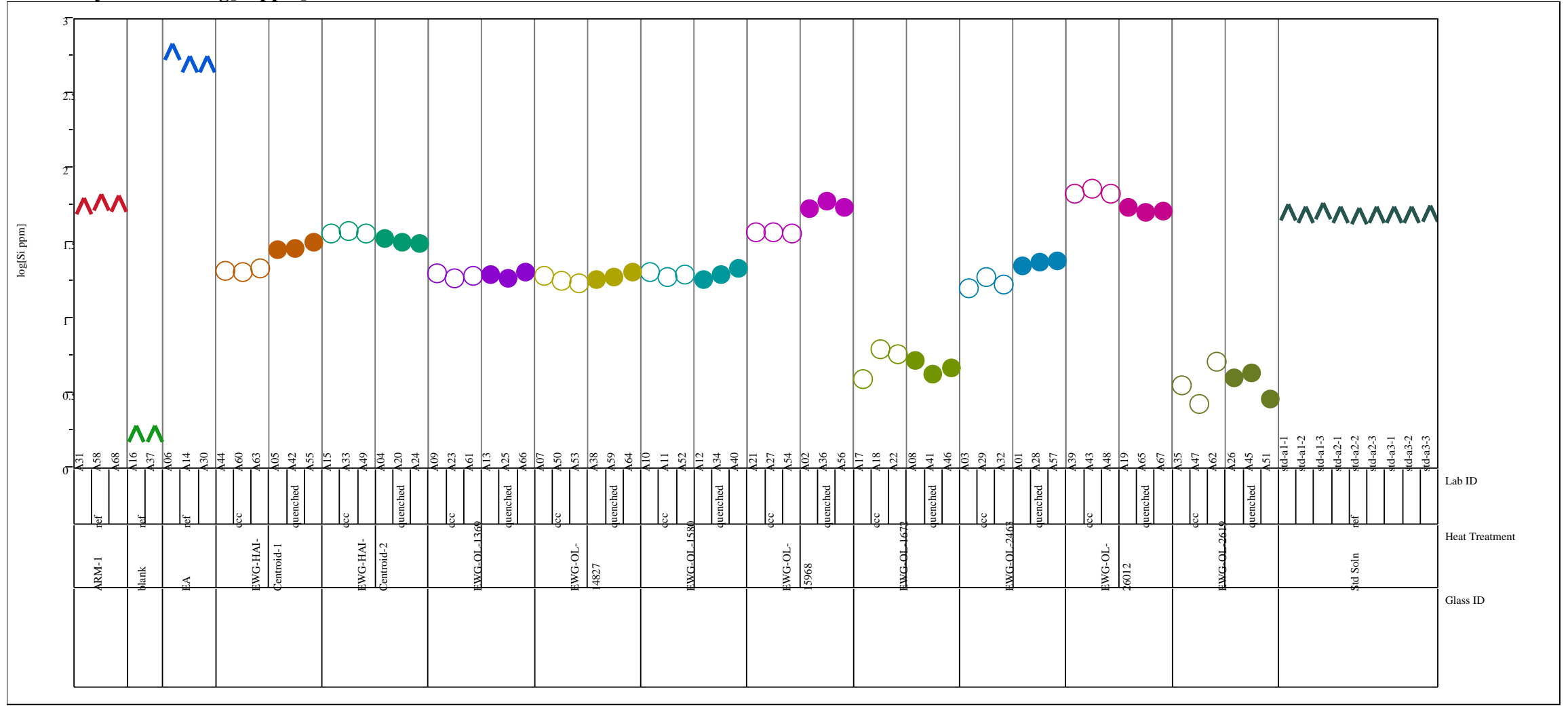




\section{Exhibit B-2. PCT Measurements for Each Glass Grouped by Heat Treatment (continued)}

Analytical Set=2

Variability Chart for $\log [\mathrm{B}$ ppm]

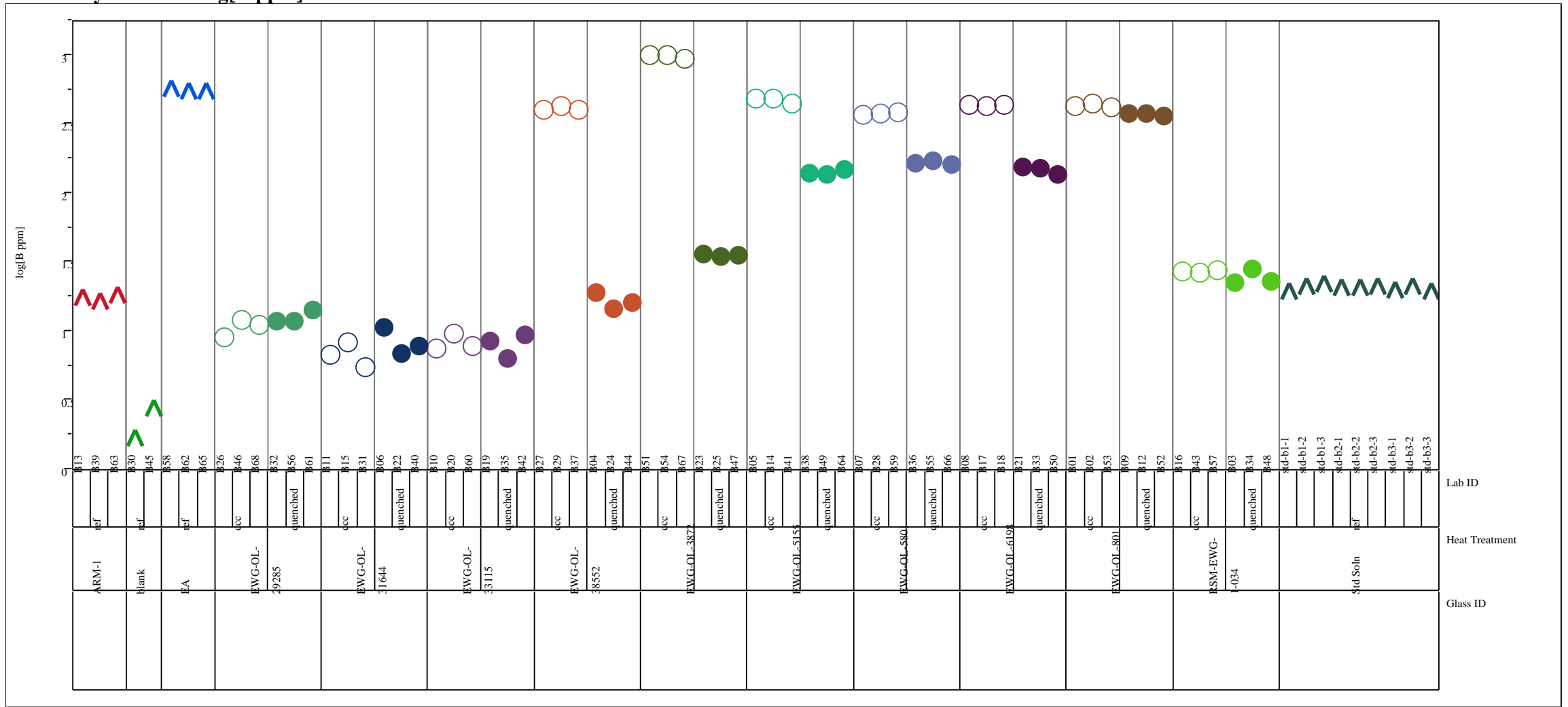




\section{Exhibit B-2. PCT Measurements for Each Glass Grouped by Heat Treatment (continued)}

Analytical Set=2

Variability Chart for log[Ca ppm $]$

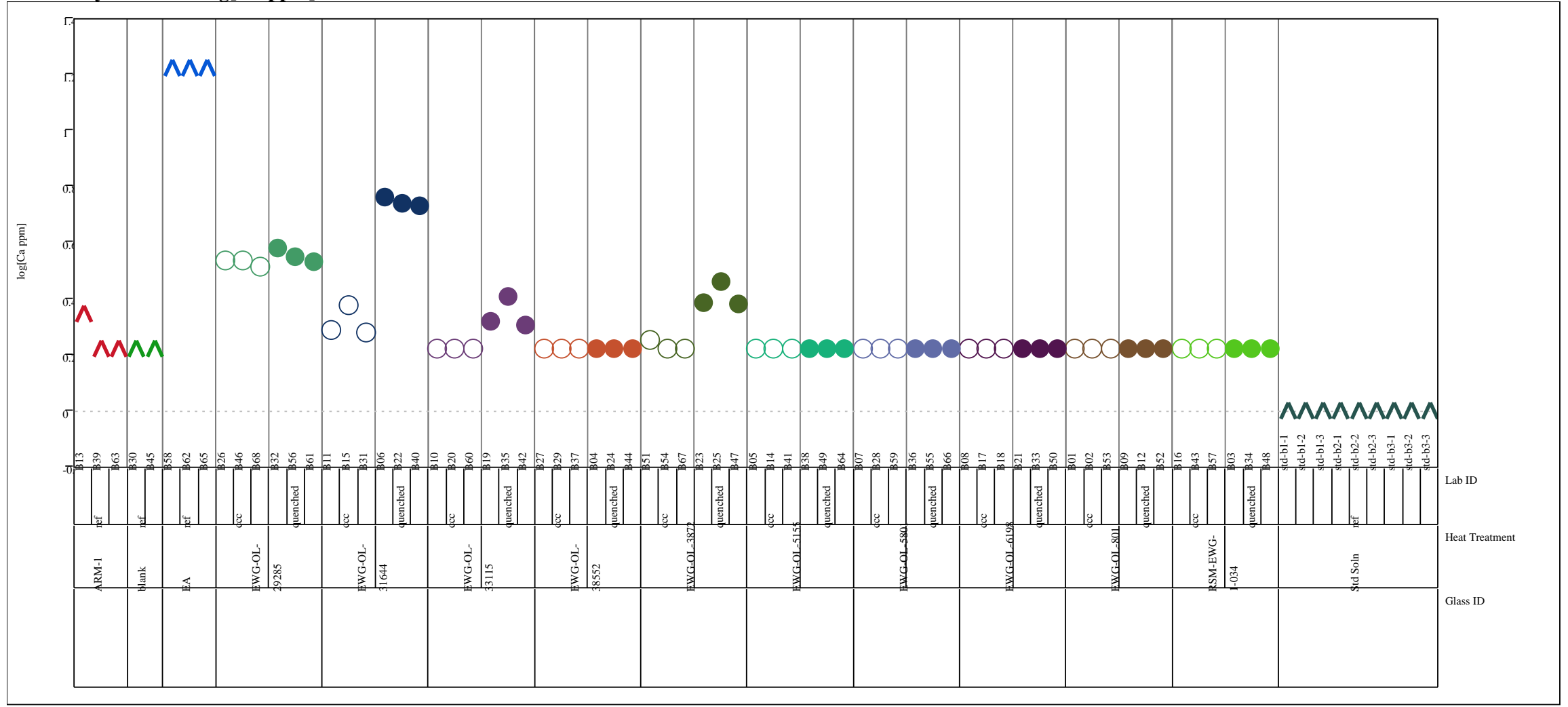




\section{Exhibit B-2. PCT Measurements for Each Glass Grouped by Heat Treatment (continued)}

Analytical Set=2

Variability Chart for $\log [\mathrm{K}$ ppm]

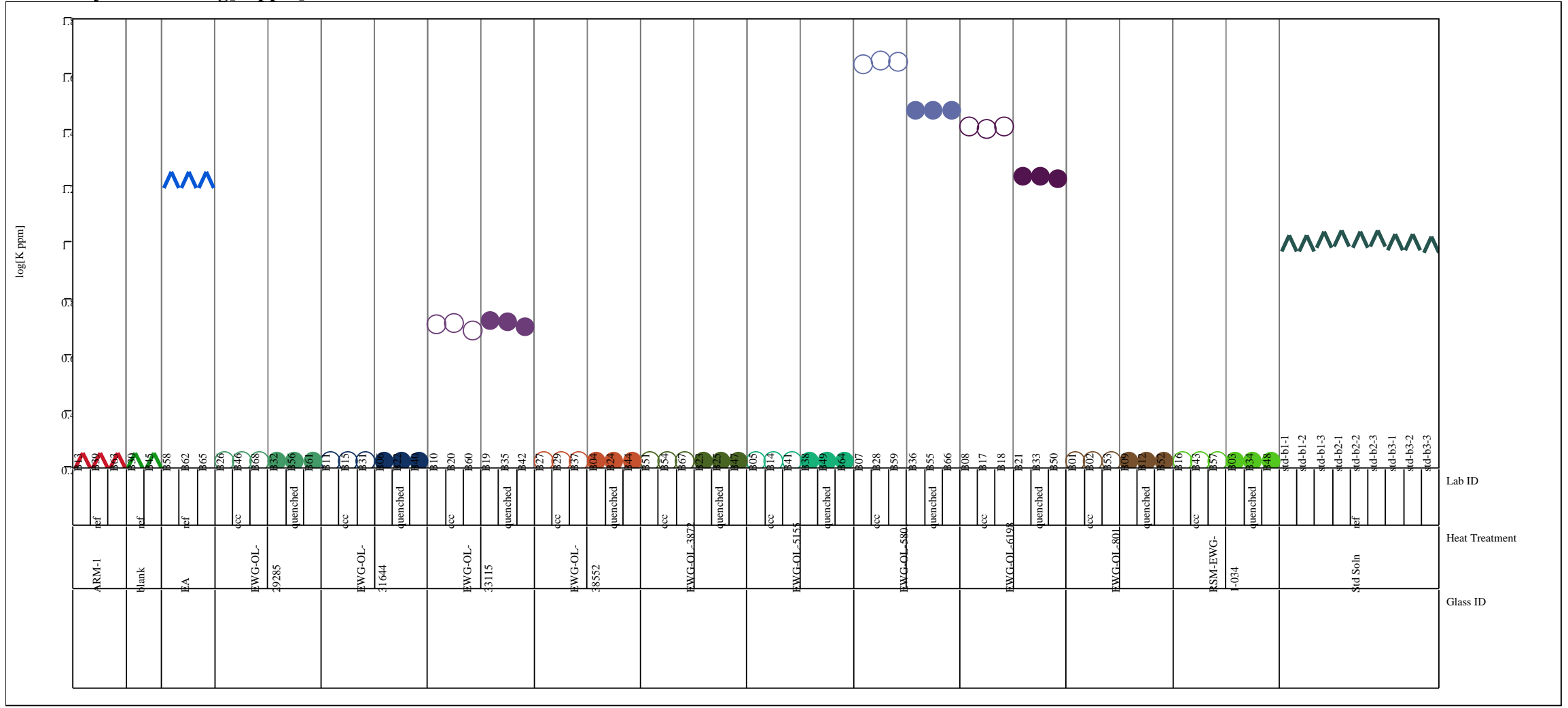




\section{Exhibit B-2. PCT Measurements for Each Glass Grouped by Heat Treatment (continued)}

Analytical Set $=2$

Variability Chart for $\log [\mathrm{Li}$ ppm]

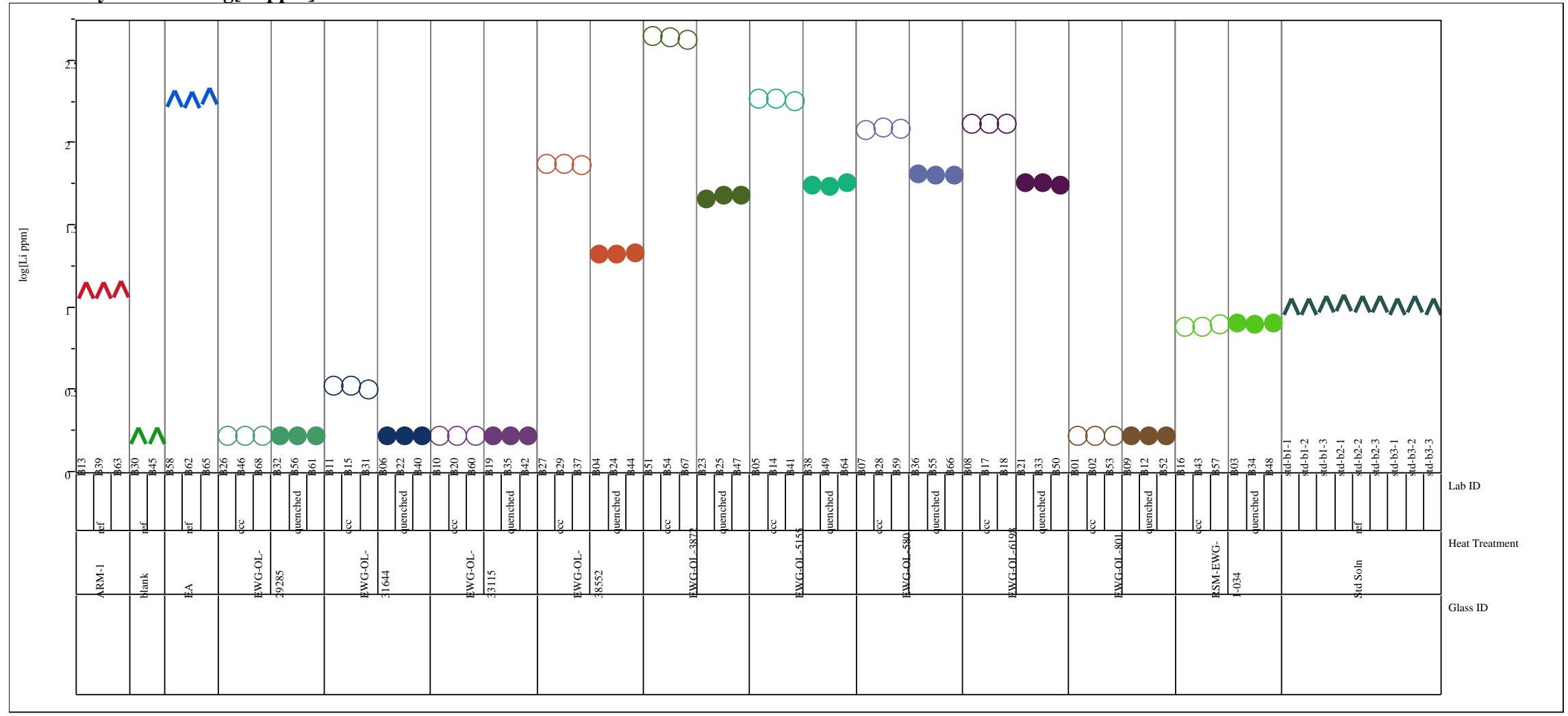




\section{Exhibit B-2. PCT Measurements for Each Glass Grouped by Heat Treatment (continued)}

Analytical Set=2

Variability Chart for $\log [\mathrm{Na}$ ppm

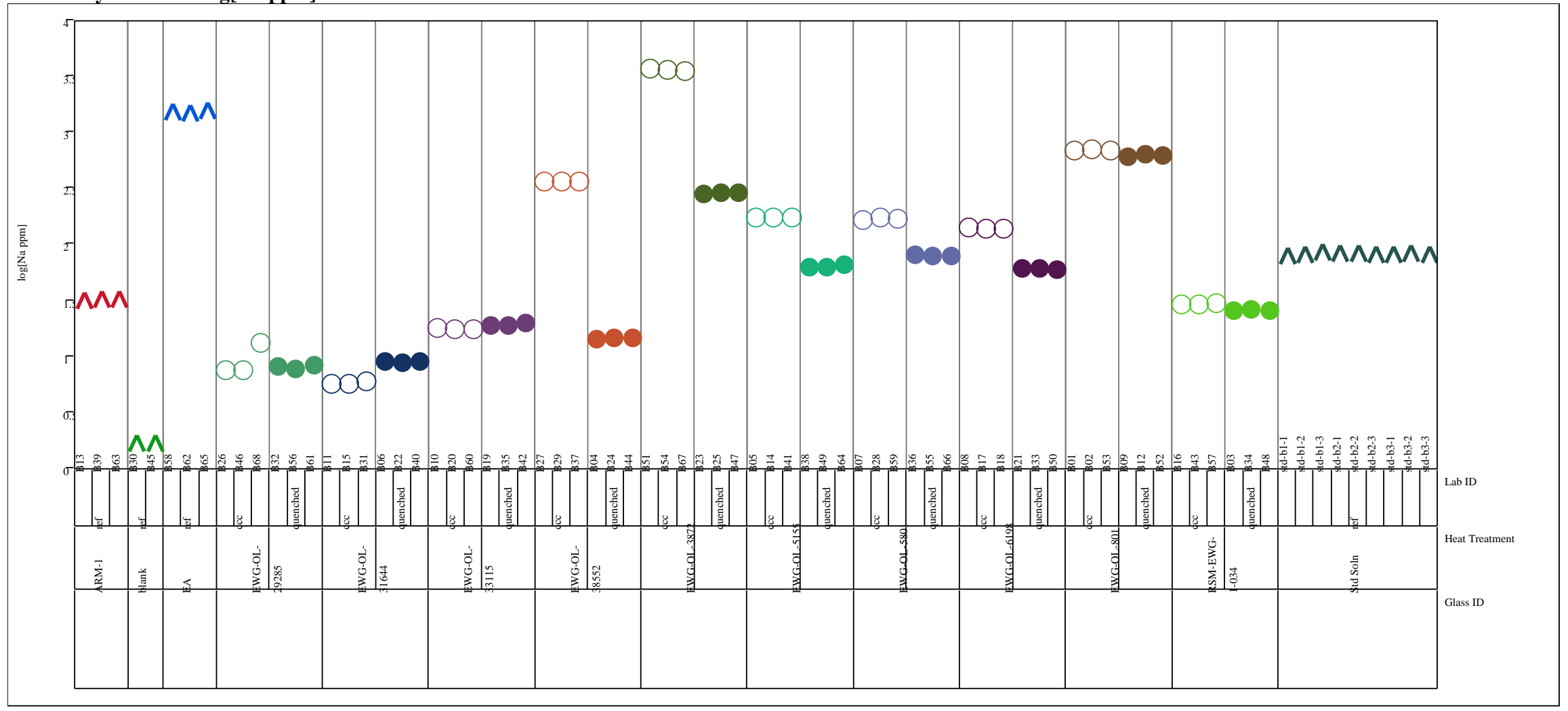




\section{Exhibit B-2. PCT Measurements for Each Glass Grouped by Heat Treatment (continued)}

Analytical Set=2

Variability Chart for $\log [\mathbf{P}$ ppm]

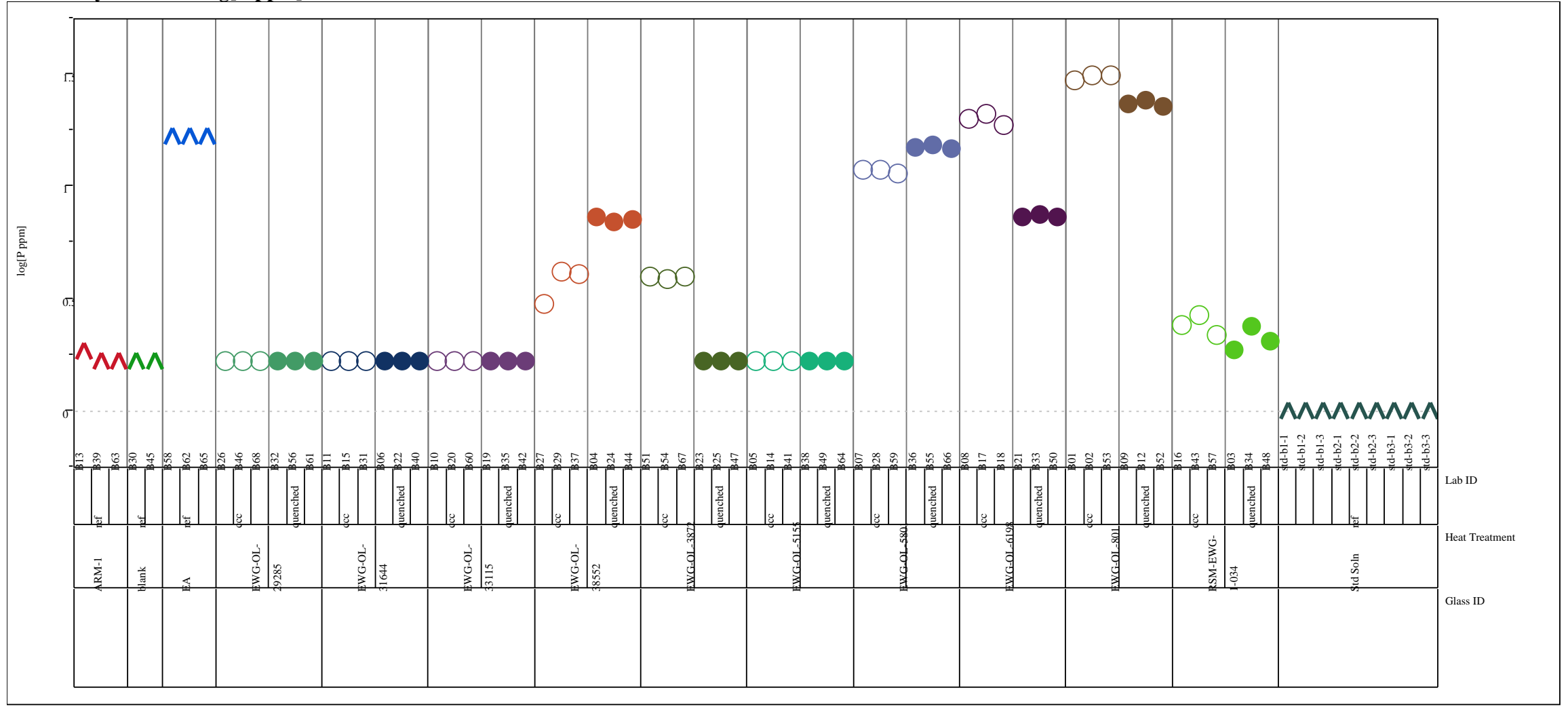




\section{Exhibit B-2. PCT Measurements for Each Glass Grouped by Heat Treatment (continued)}

Analytical Set $=2$

Variability Chart for $\log [\mathrm{Si}$ ppm]

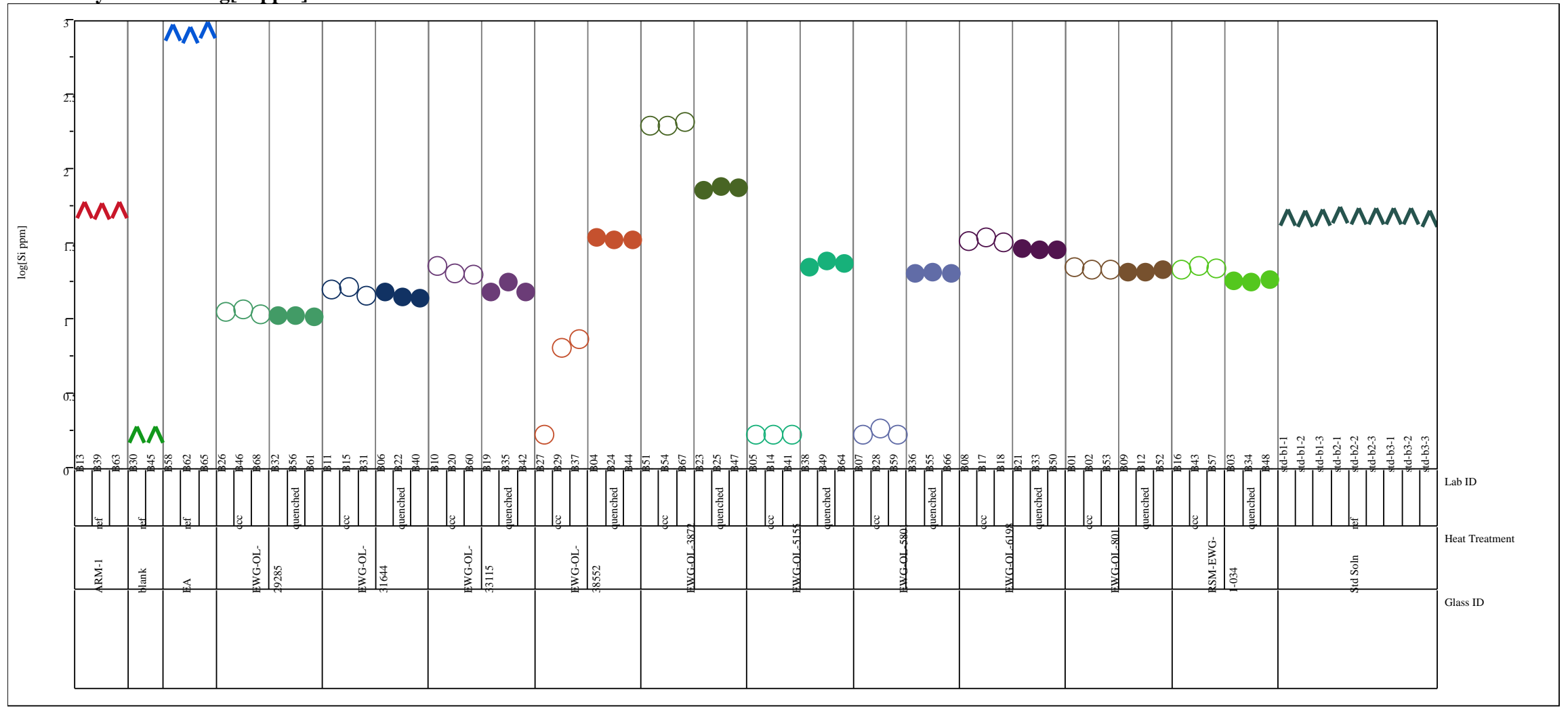


Exhibit B-3. Measurements of the Solution Standard by Analytical Block

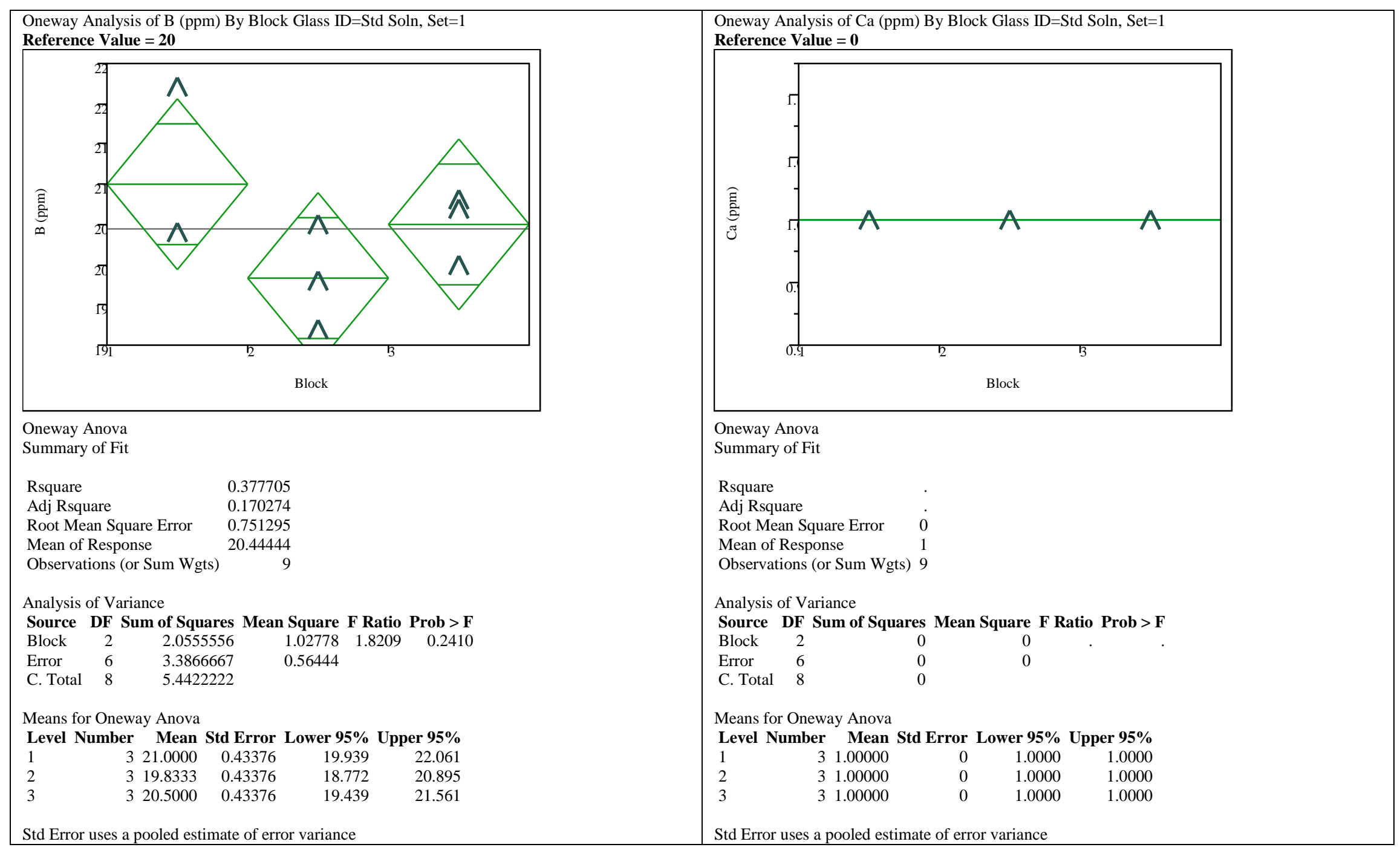


Exhibit B-3. Measurements of the Solution Standard by Analytical Block (continued)

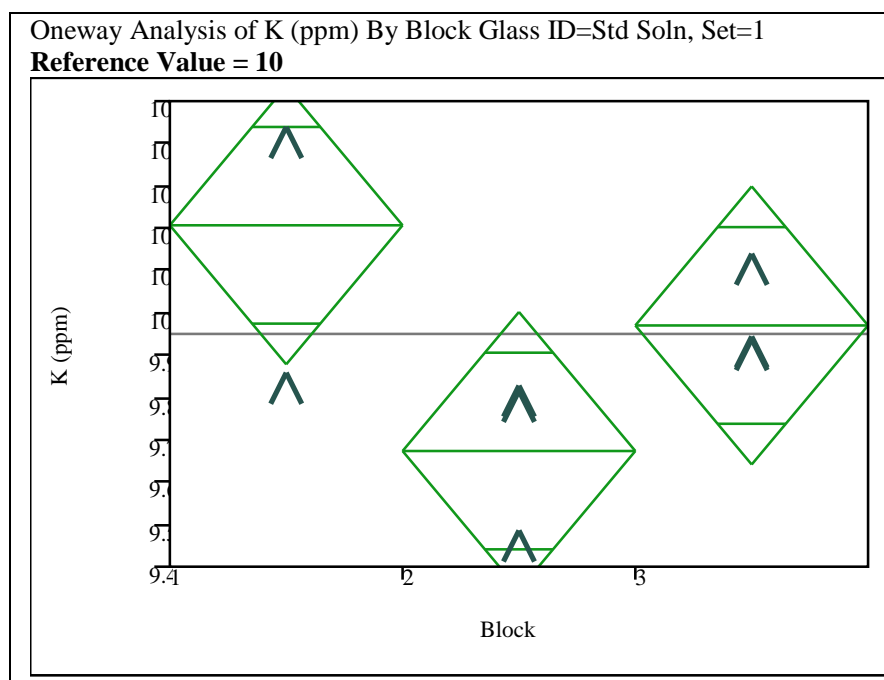

Oneway Anova

Summary of Fit

$$
\begin{array}{lr}
\text { Rsquare } & 0.569013 \\
\text { Adj Rsquare } & 0.42535 \\
\text { Root Mean Square Error } & 0.23257 \\
\text { Mean of Response } & 9.95 \\
\text { Observations (or Sum Wgts) } & 9
\end{array}
$$

Analysis of Variance

Source DF Sum of Squares Mean Square F Ratio Prob $>$ F

$\begin{array}{llllll}\text { Block } & 2 & 0.42846667 & 0.214233 & 3.9608 & 0.0801\end{array}$

$\begin{array}{llll}\text { Error } & 6 & 0.32453333 & 0.054089\end{array}$

$\begin{array}{lll}\text { C. Total } & 8 & 0.75300000\end{array}$

$$
0.054089
$$

Means for Oneway Anova

Level Number Mean Std Error Lower 95\% Upper 95\%

$$
\begin{array}{llrrrr}
1 & 3 & 10.2067 & 0.13427 & 9.8781 & 10.535 \\
2 & 3 & 9.6733 & 0.13427 & 9.3448 & 10.002 \\
3 & 3 & 9.9700 & 0.13427 & 9.6414 & 10.299
\end{array}
$$

Std Error uses a pooled estimate of error variance

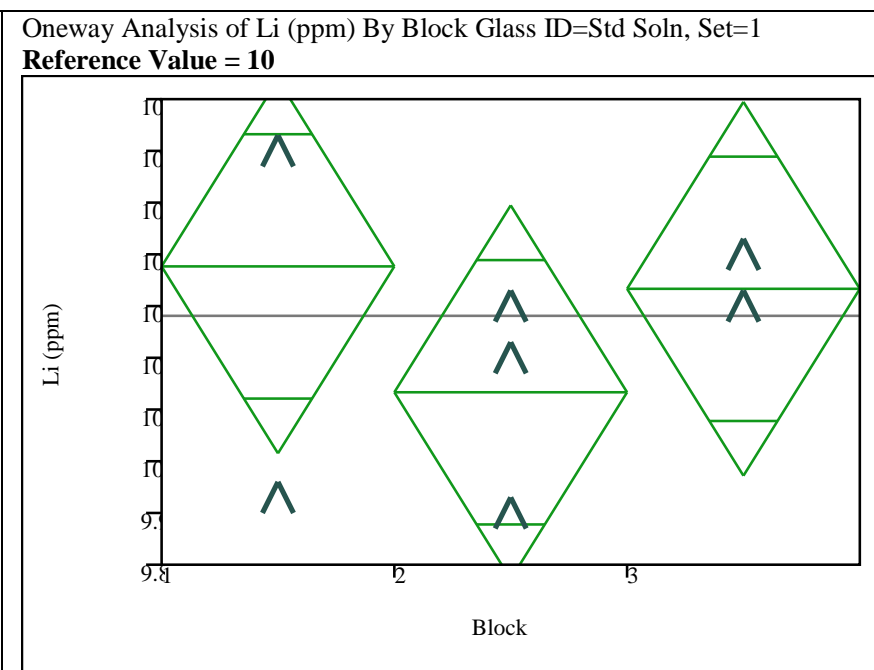

Oneway Anova

Summary of Fit

$$
\begin{array}{lr}
\text { Rsquare } & 0.204762 \\
\text { Adj Rsquare } & -0.06032 \\
\text { Root Mean Square Error } & 0.255799 \\
\text { Mean of Response } & 10.28111 \\
\text { Observations (or Sum Wgts) } & 9
\end{array}
$$

Analysis of Variance

Source DF Sum of Squares Mean Square F Ratio Prob > F

$\begin{array}{lrrrrr}\text { Block } & 2 & 0.10108889 & 0.050544 & 0.7725 & 0.5029\end{array}$

$\begin{array}{lll}\text { Error } & 6 & 0.39260000\end{array}$

C. Total $8 \quad 0.49368889$

0.065433

Means for Oneway Anova

Level Number Mean Std Error Lower 95\% Upper 95\%

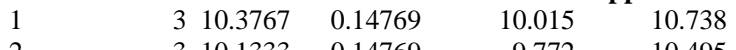

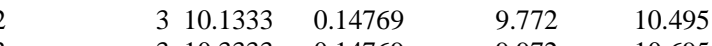

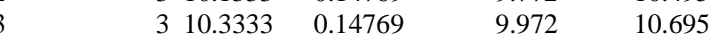

Std Error uses a pooled estimate of error variance 
Exhibit B-3. Measurements of the Solution Standard by Analytical Block (continued)

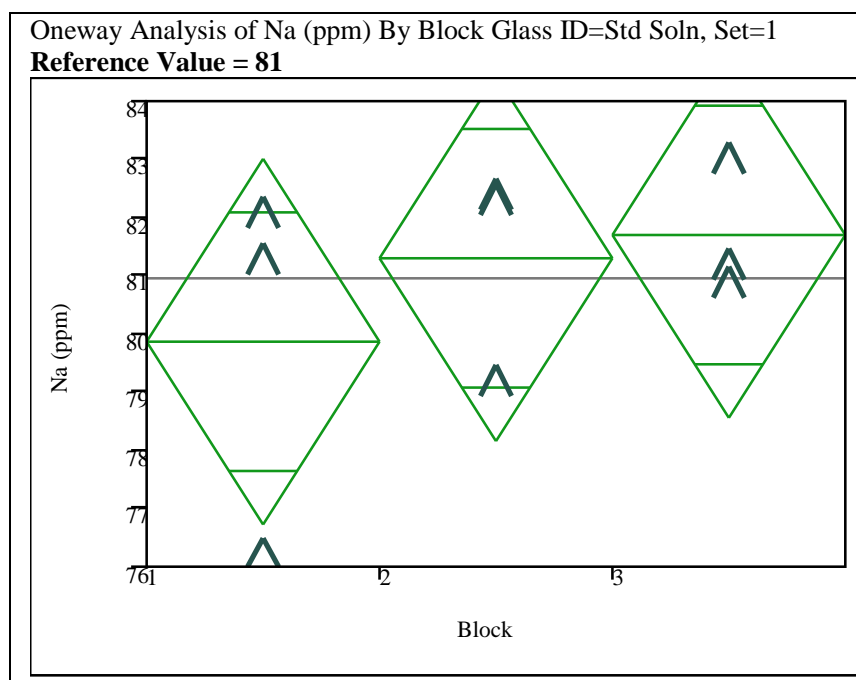

Oneway Anova

Summary of Fi

Rsquare

0.158117

Adj Rsquare

$-0.12251$

Root Mean Square Error $\quad 2.22436$

Mean of Response 80.95556

Observations (or Sum Wgts)

Analysis of Variance

Source DF Sum of Squares Mean Square F Ratio Prob $>$ F

$\begin{array}{lrrrrr}\text { Block } & 2 & 5.575556 & 2.78778 & 0.5634 & 0.5967\end{array}$

$\begin{array}{lll}\text { Error } & 6 & 29.686667\end{array}$

2.78778
4.94778

C. Total 8

35.262222

Means for Oneway Anova

Level Number Mean Std Error Lower 95\% Upper 95\%

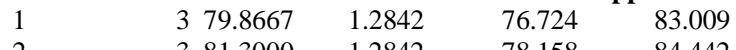

$\begin{array}{lllll}2 & 381.3000 & 1.2842 & 78.158 & 84.442\end{array}$

Std Error uses a pooled estimate of error variance

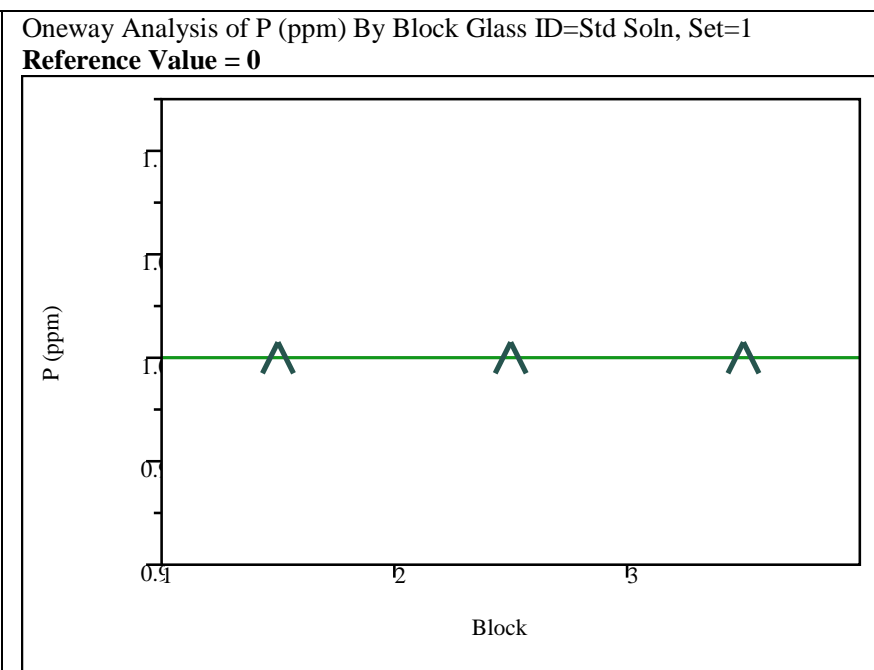

Oneway Anova

Summary of Fit

\section{Rsquare}

Adj Rsquare

Root Mean Square Error

Mean of Response

Observations (or Sum Wgts) 9

Analysis of Variance

Source DF Sum of Squares Mean Square F Ratio Prob $>$ F

$\begin{array}{llll}\text { Block } & 2 & 0 & 0\end{array}$

Error $6 \quad 0$

C. Total 8

0

Means for Oneway Anova

Level Number Mean Std Error Lower 95\% Upper 95\%

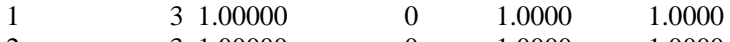

$\begin{array}{rrrrr}2 & 31.00000 & 0 & 1.0000 & 1.0000\end{array}$

Std Error uses a pooled estimate of error variance 
Exhibit B-3. Measurements of the Solution Standard by Analytical Block (continued)

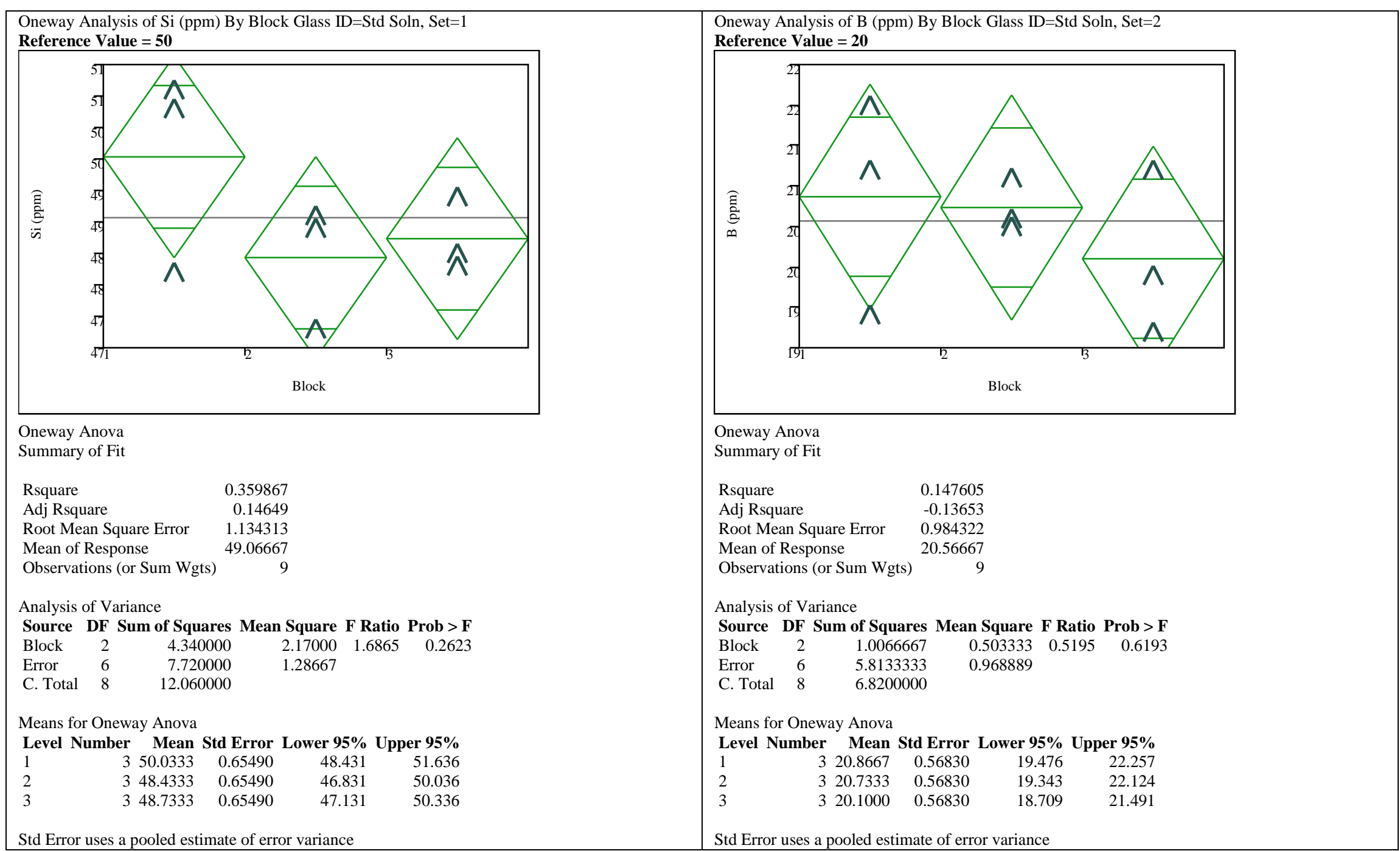


Exhibit B-3. Measurements of the Solution Standard by Analytical Block (continued)

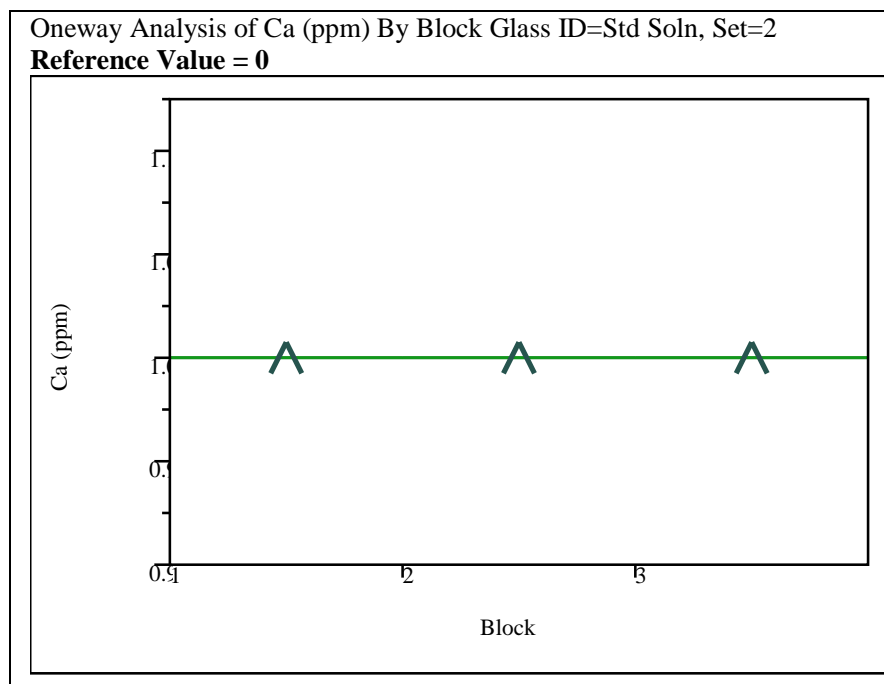

Oneway Anova

Summary of Fit

Rsquare

Adj Rsquare

Root Mean Square Error $\quad \dot{0}$

Mean of Response

Observations (or Sum Wgts) 9

Analysis of Variance

Source DF Sum of Squares Mean Square F Ratio Prob $>$ F

$\begin{array}{llll}\text { Block } & 2 & 0 & 0\end{array}$

$\begin{array}{llll}\text { Error } & 6 & 0 & 0\end{array}$

C. Total 8

Means for Oneway Anova

Level Number Mean Std Error Lower 95\% Upper 95\%

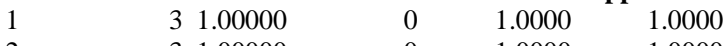

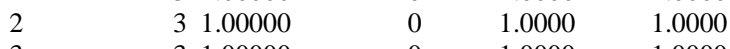

$\begin{array}{llll}31.00000 & 0 & 1.0000 & 1.0000\end{array}$

Std Error uses a pooled estimate of error variance

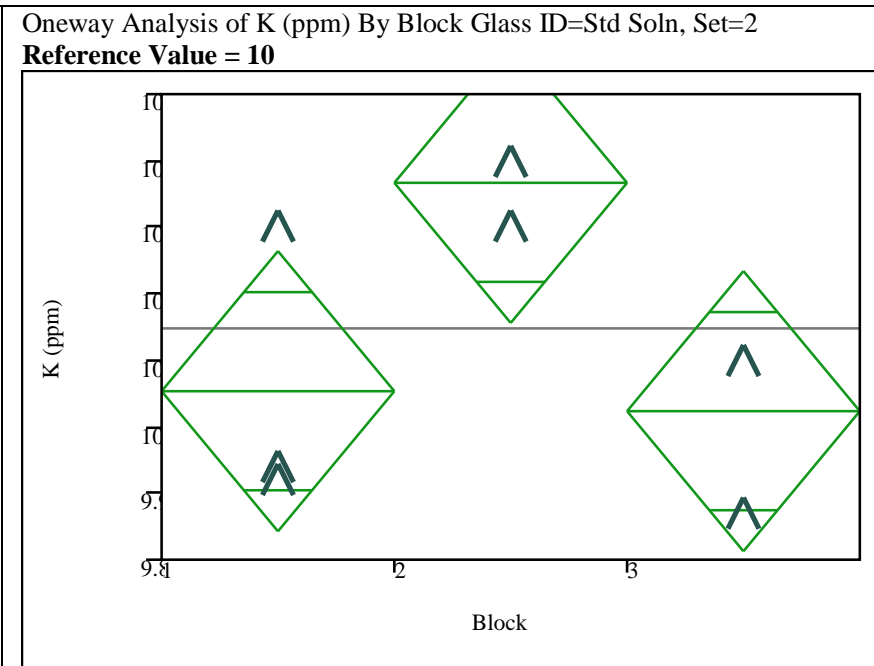

Oneway Anova

Summary of Fit

Rsquare

Adj Rsquare

Root Mean Square Error

Mean of Response

0.619244

0.492325

0.149108

Observations (or Sum Wgts)

10.14778
9

Analysis of Variance

Source DF Sum of Squares Mean Square F Ratio Prob $>$ F

$\begin{array}{llllll}\text { Block } & 2 & 0.21695556 & 0.108478 & 4.8791 & 0.0552\end{array}$

Error 60.13340000

0.108478

C. Total $8 \quad 0.35035556$

Means for Oneway Anova

Level Number Mean Std Error Lower 95\% Upper 95\%

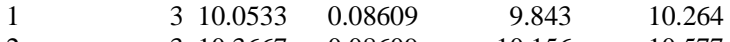

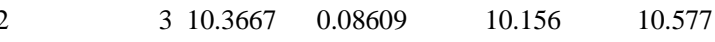

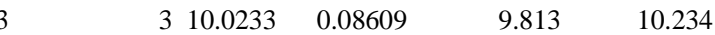

Std Error uses a pooled estimate of error variance 
Exhibit B-3. Measurements of the Solution Standard by Analytical Block (continued)

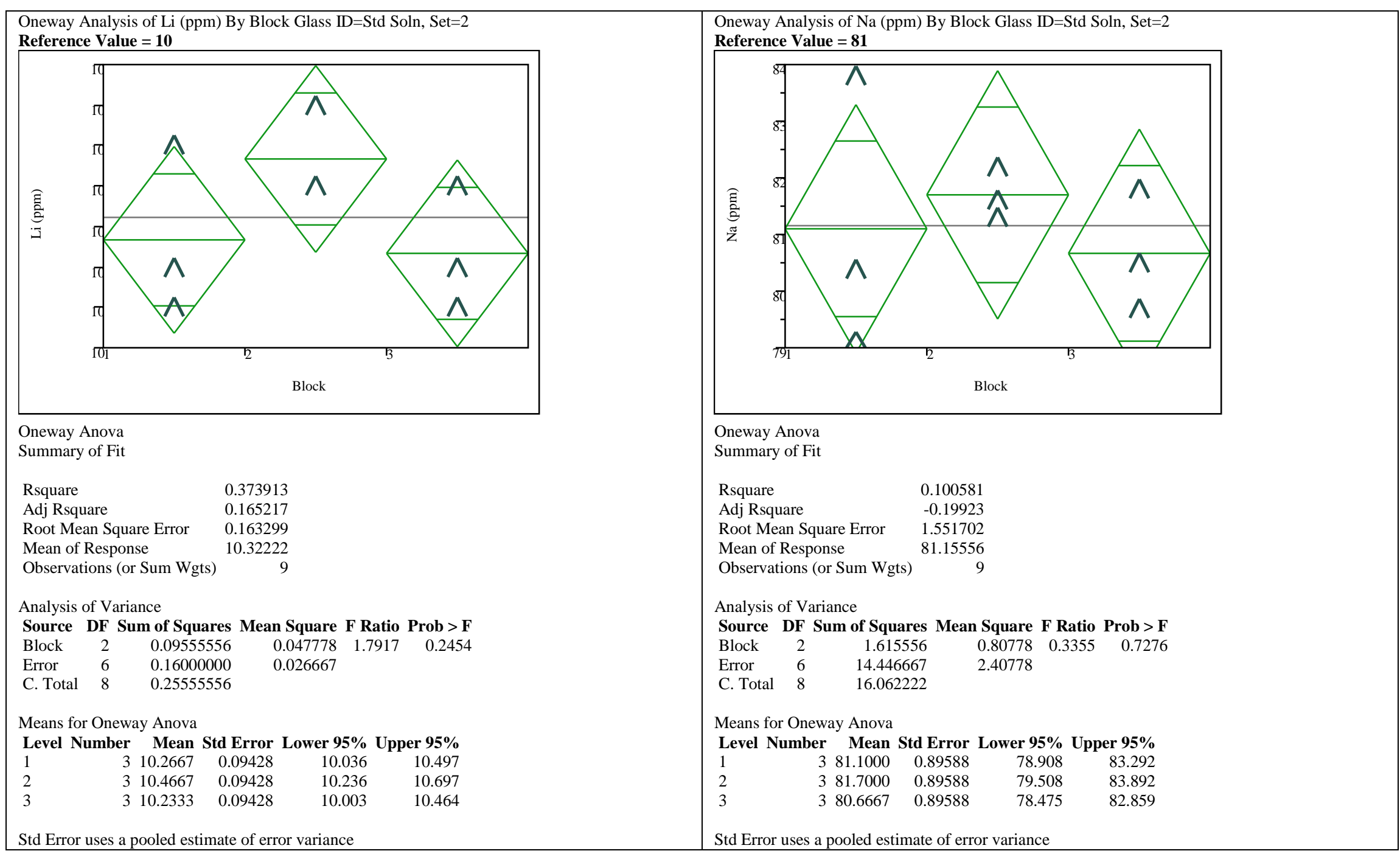


Exhibit B-3. Measurements of the Solution Standard by Analytical Block (continued)

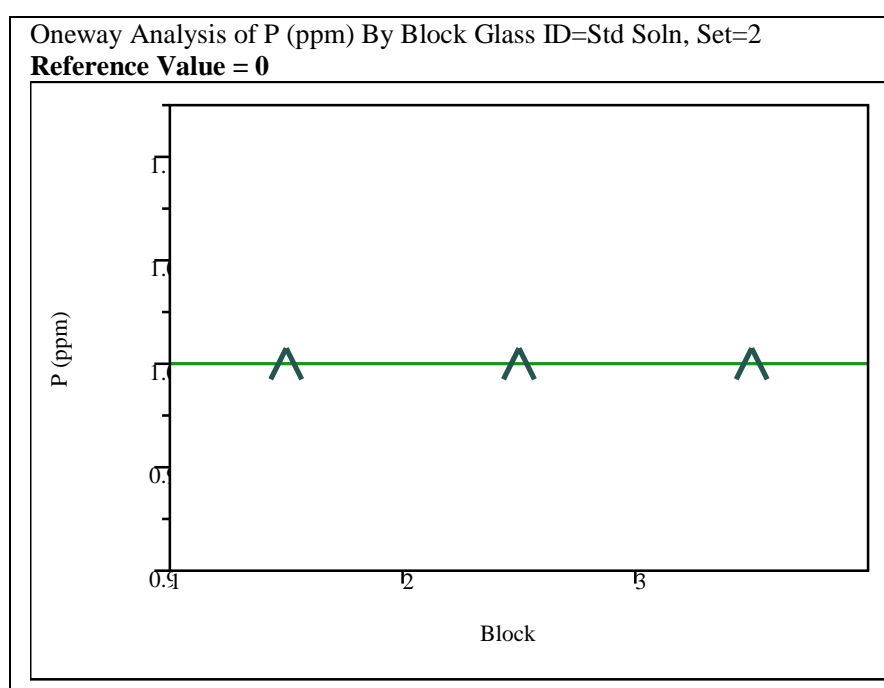

Oneway Anova

Summary of Fit

Rsquare

Adj Rsquare

Root Mean Square Error $\quad \dot{0}$

Mean of Response

Observations (or Sum Wgts) 9

Analysis of Variance

Source DF Sum of Squares Mean Square F Ratio Prob $>$ F

$\begin{array}{llll}\text { Block } & 2 & 0 & 0\end{array}$

$\begin{array}{llll}\text { Error } & 6 & 0 & 0\end{array}$

C. Total 8

Means for Oneway Anova

Level Number Mean Std Error Lower 95\% Upper 95\%

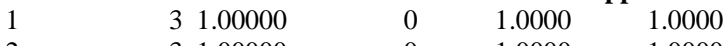

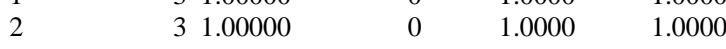

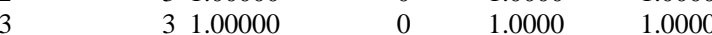

Std Error uses a pooled estimate of error variance

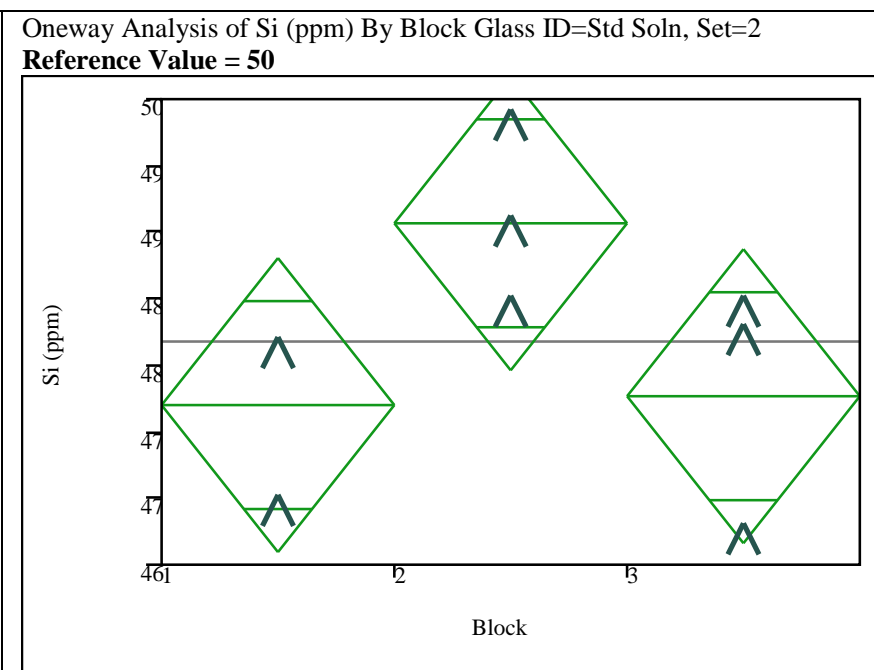

Oneway Anova

Summary of Fit

$\begin{array}{lr}\text { Rsquare } & 0.492322 \\ \text { Adj Rsquare } & 0.323096 \\ \text { Root Mean Square Error } & 0.782446 \\ \text { Mean of Response } & 48.17778 \\ \text { Observations (or Sum Wgts) } & 9\end{array}$

Observations (or Sum Wgs)

Analysis of Variance

Source DF Sum of Squares Mean Square F Ratio Prob $>$ F

$\begin{array}{llllll}\text { Block } & 2 & 3.5622222 & 1.78111 & 2.9093 & 0.1308\end{array}$

$\begin{array}{llll}\text { Block } & 2 & 3.5622222 & 1.78111 \\ \text { Error } & 6 & 3.6733333 & 0.61222\end{array}$

C. Total $8 \quad 7.2355556$

Means for Oneway Anova

Level Number Mean Std Error Lower 95\% Upper 95\%

$\begin{array}{lllll}1 & 347.7000 & 0.45175 & 46.595 & 48.805\end{array}$

$\begin{array}{lllll}2 & 349.0667 & 0.45175 & 47.961 & 50.172\end{array}$

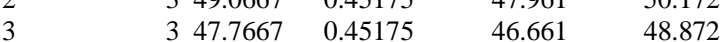

Std Error uses a pooled estimate of error variance 
Exhibit B-4. Normalized PCT Results by Heat Treatment by Compositional View for Glass

Variability Chart for log NL[B (g/L)]

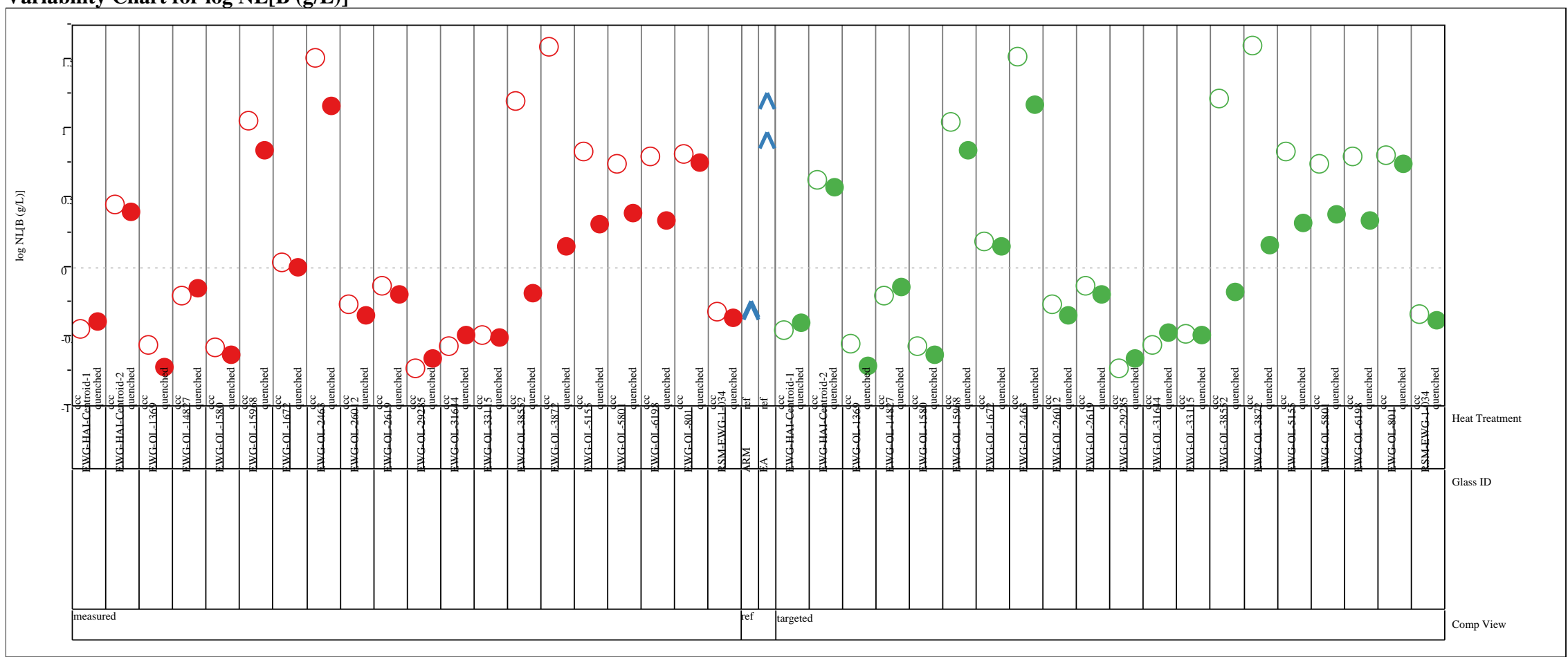


Exhibit B-4. Normalized PCT Results by Heat Treatment by Compositional View for Glass (continued) Variability Chart for $\log$ NL[Ca (g/L)]

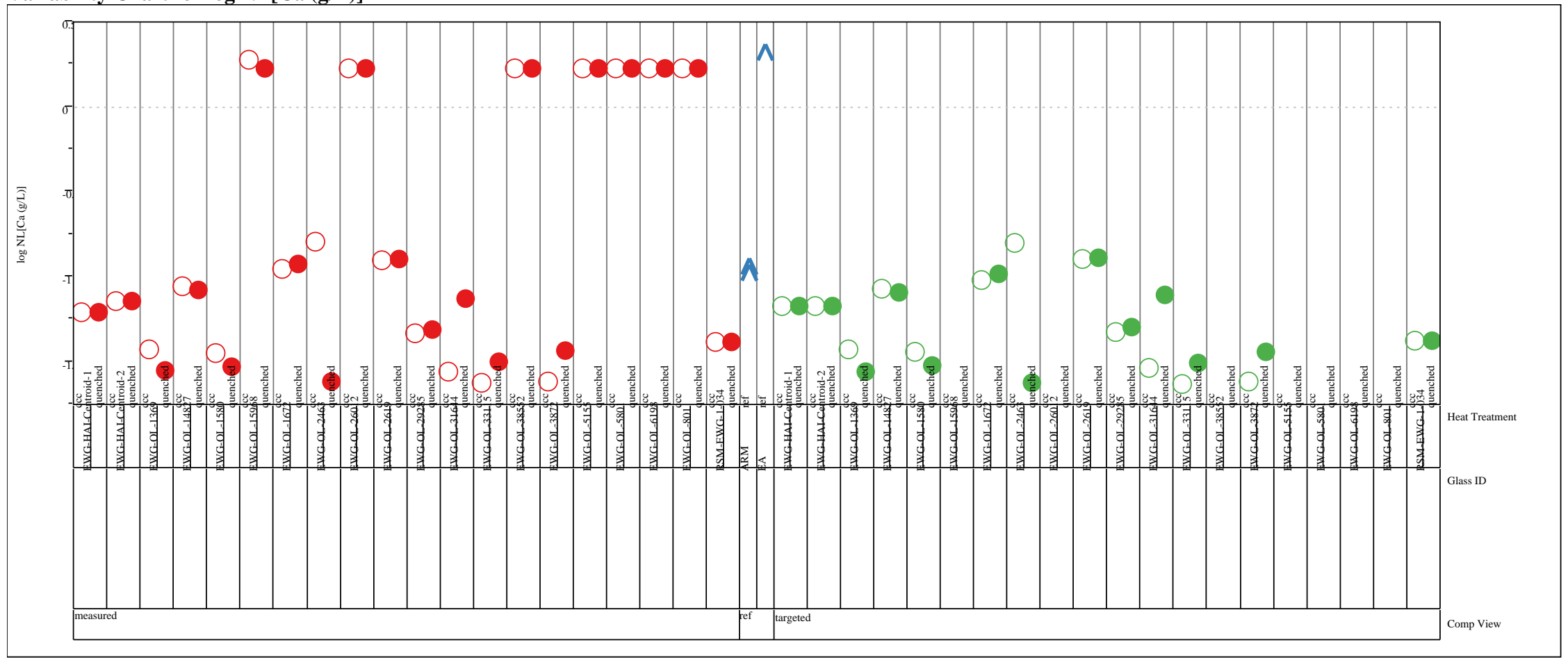


Exhibit B-4. Normalized PCT Results by Heat Treatment by Compositional View for Glass (continued) Variability Chart for $\log$ NL[K (g/L)]

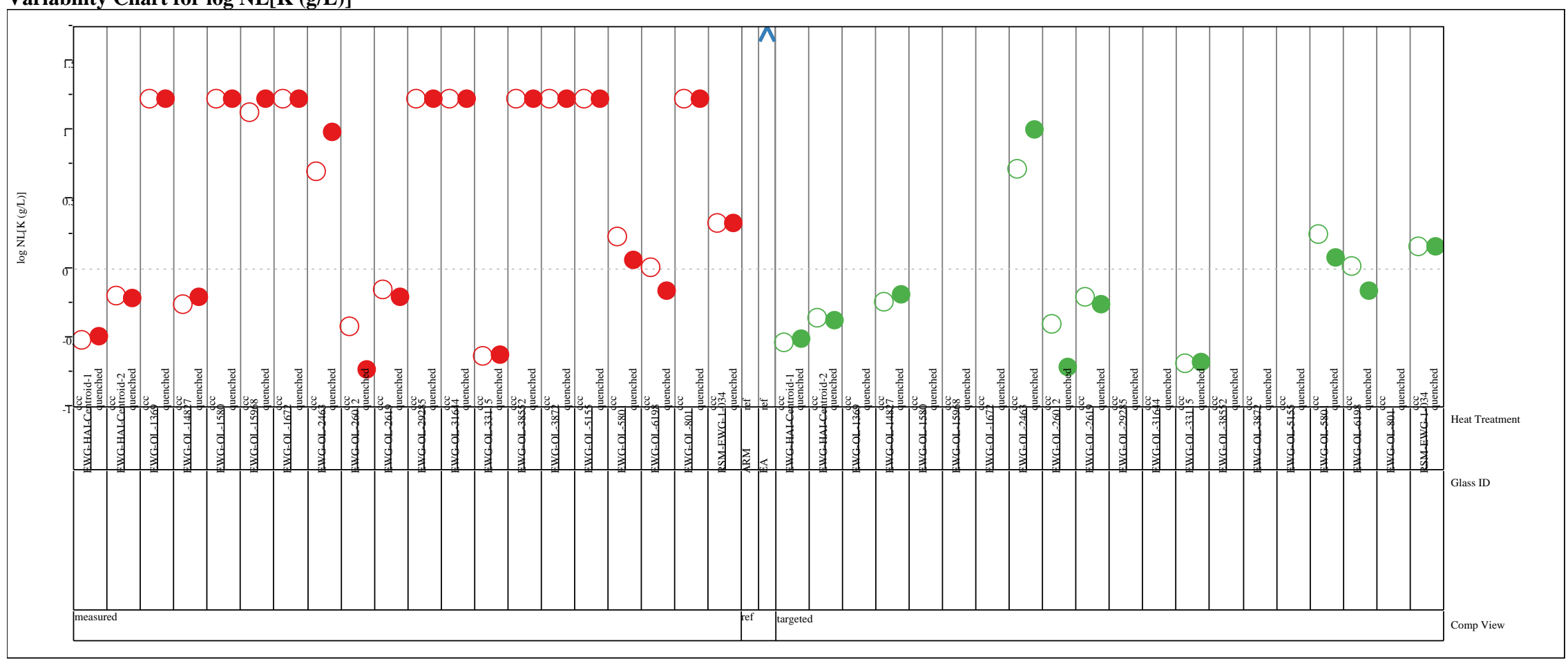


Exhibit B-4. Normalized PCT Results by Heat Treatment by Compositional View for Glass (continued) Variability Chart for $\log N L[\mathrm{Li}(\mathrm{g} / \mathrm{L})]$

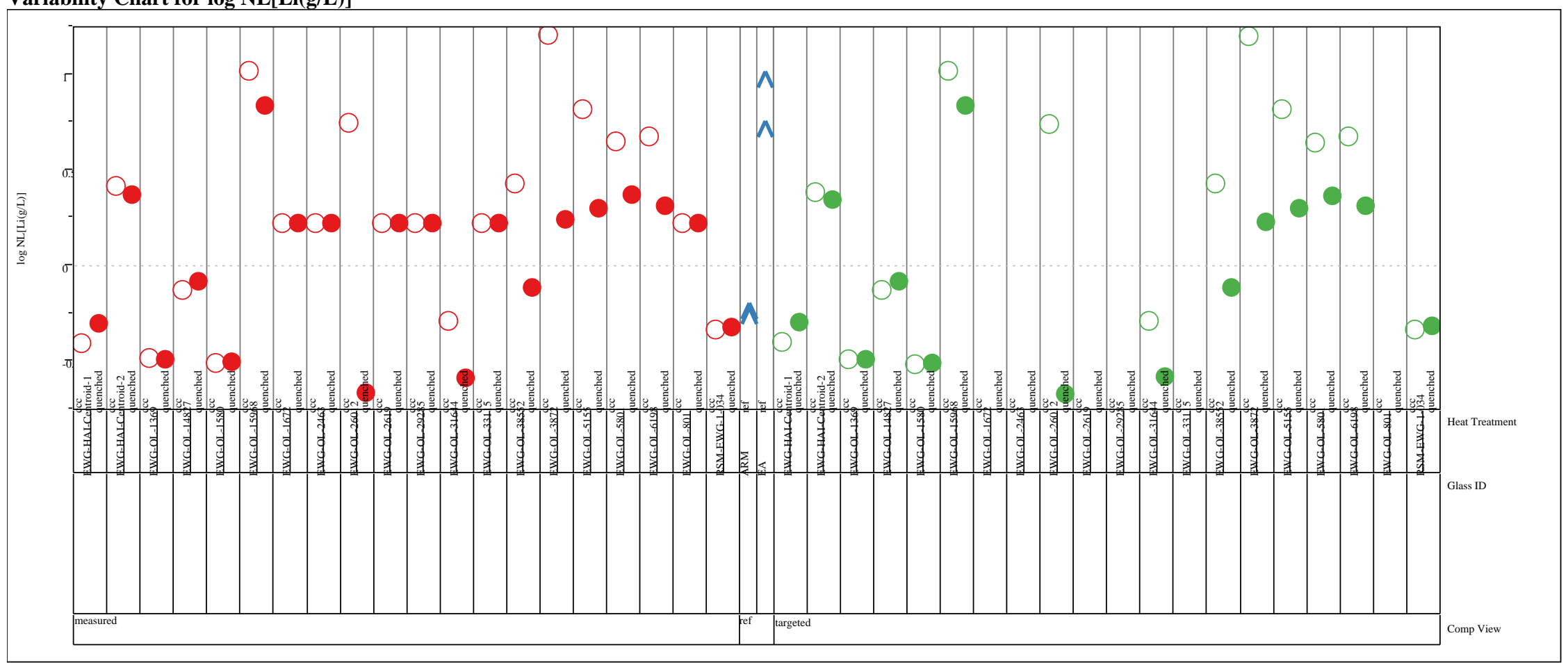


Exhibit B-4. Normalized PCT Results by Heat Treatment by Compositional View for Glass (continued) Variability Chart for $\log$ NL[Na (g/L)]

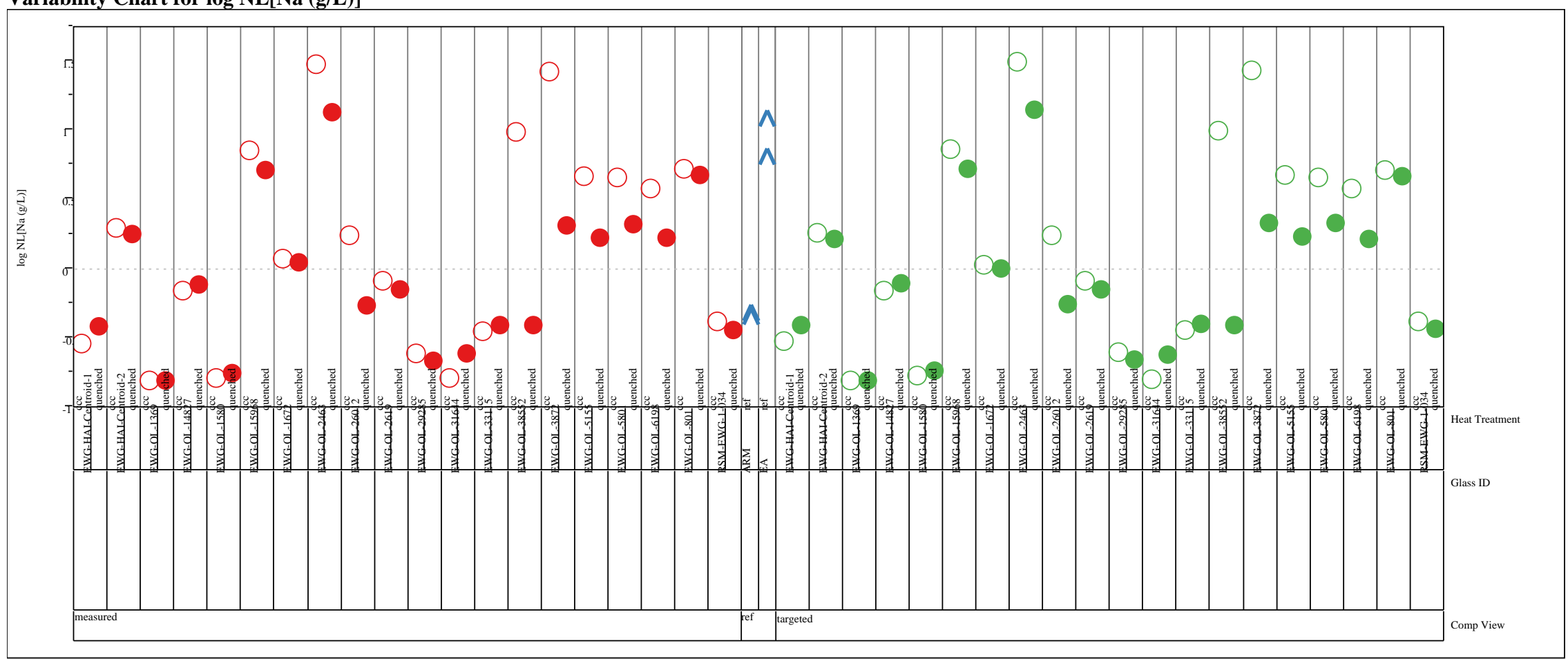


Exhibit B-4. Normalized PCT Results by Heat Treatment by Compositional View for Glass (continued) Variability Chart for $\log$ NL[P (g/L)]

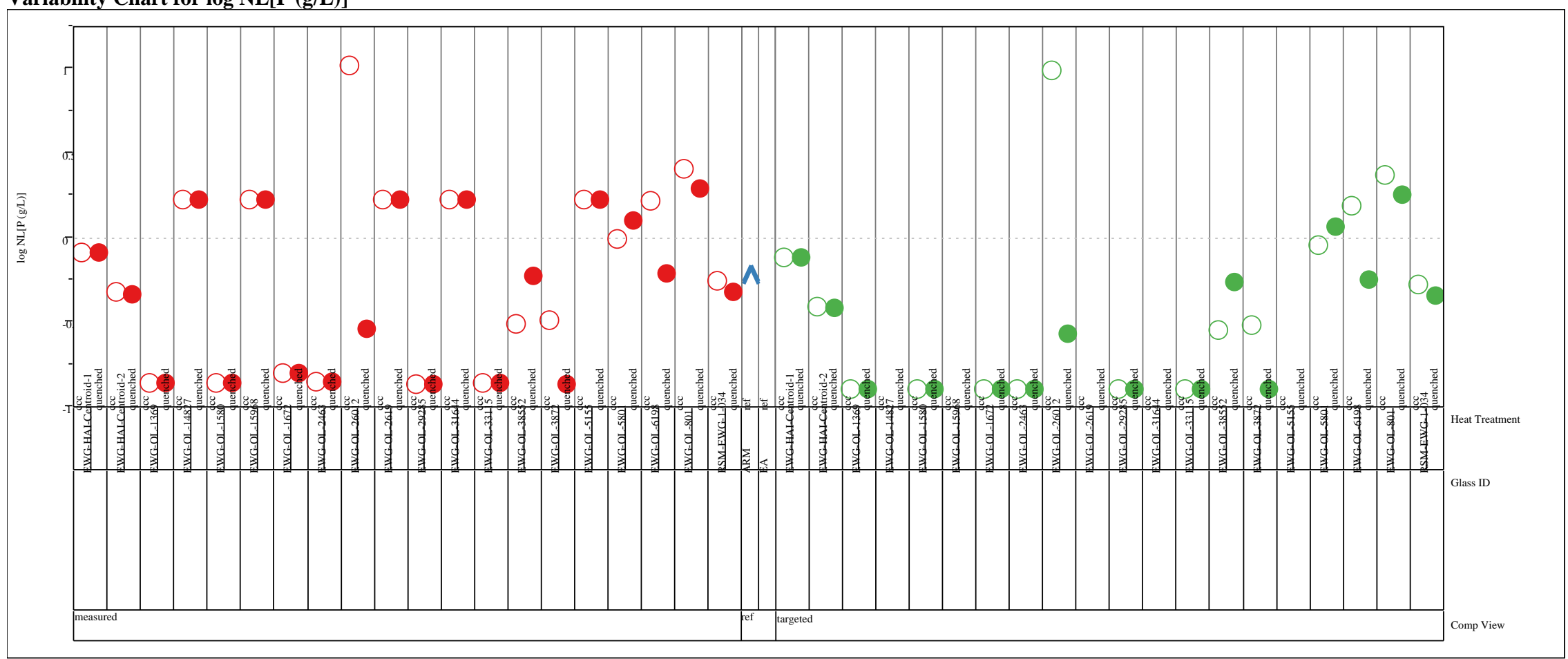


Exhibit B-4. Normalized PCT Results by Heat Treatment by Compositional View for Glass (continued) Variability Chart for log NL[Si (g/L)]

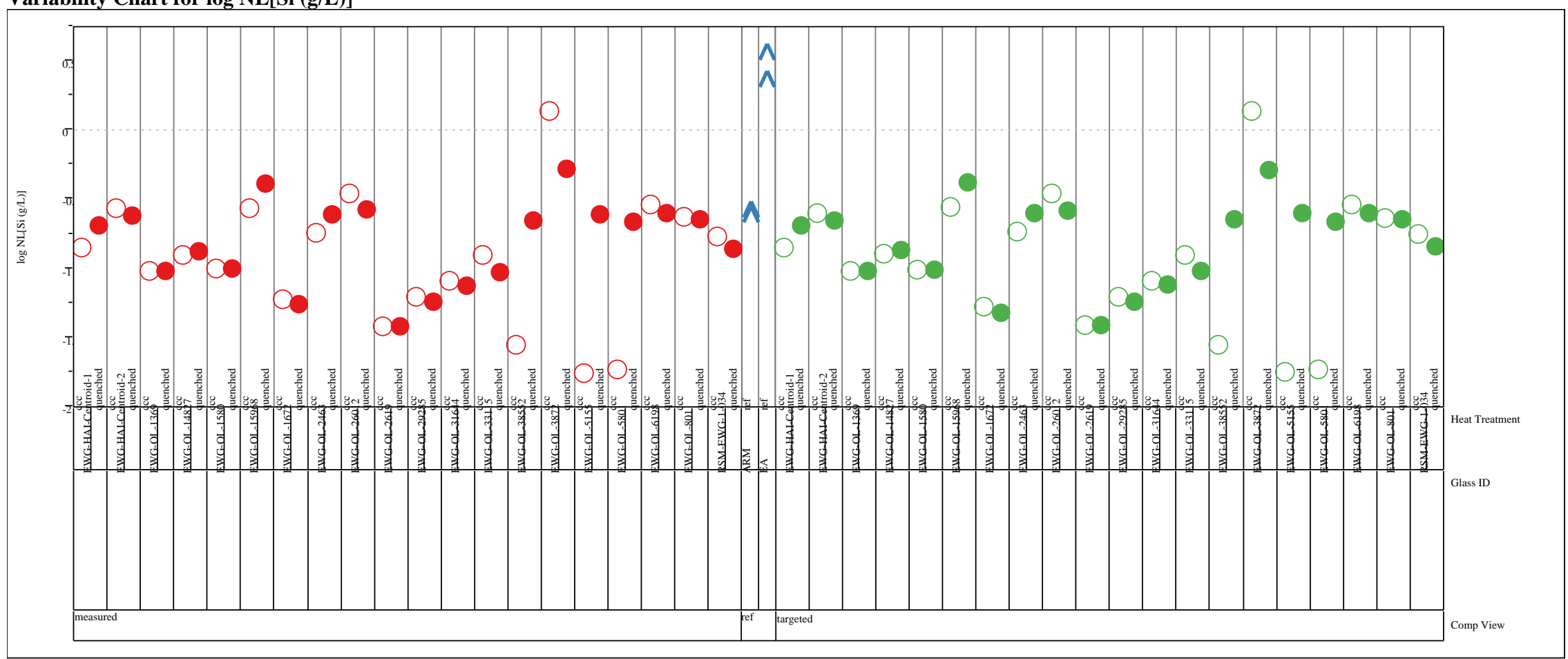




\section{Distribution:}

J. W. Amoroso, 999-W

T. B. Brown, 773-A

J. V. Crum, PNNL

W. A. Drown, 773-41

T. B. Edwards, 999-W

S. D. Fink, 773-A

K. M. Fox, 999-W

C. C. Herman, 773-A

E. N. Hoffman, 999-W

F. C. Johnson, 999-W

A. A. Kruger, DOE-ORP

S. L. Marra, 773-A

D. H. McGuire, 999-W

D. K. Peeler, 999-W

F. M. Pennebaker, 773-42A

J. Matyáš, PNNL

M. J. Schweiger, PNNL

J. D. Vienna, PNNL

W. R. Wilmarth, 773-A

Records Administration (EDWS) 